\title{
Synthesis, Spectral and Biological Studies of Some Salicylidene-Trimethoprim Schiff Base Complexes of Aryltellurium(IV)
}

\author{
DEEPAK, K. K. VERMA and SAPANA GARG* \\ Department of Chemistry, Maharshi Dayanand University, Rohtak-124001, India \\ sapanagarg1511@gmail.com
}

Received 14 February 2017 / Accepted 2 March 2017

\begin{abstract}
A novel monobasic tridentate Schiff base salicylidene-trimethoprim, Sal-TMP, synthesized from trimethoprim and salicylaldehyde, form stable complexes with aryltellurium(IV) trichlorides and diaryltellurium(IV) dichlorides of the type Sal-TMP.ArTeCl ${ }_{2}$ and $\mathrm{Sal}_{-} \mathrm{TMP}_{\mathrm{Ar}} \mathrm{AeCl}$ (where $\mathrm{Ar}=p$-methoxyphenyl, $p$-ethoxyphenyl, $p$-hydroxyphenyl and 3-methyl-4-hydroxyphenyl). These have been characterized by elemental analyses, molar conductance, IR and ${ }^{1} \mathrm{H}$ NMR spectroscopy. The spectral studies predict the bonding of ligand through phenolic oxygen of Schiff base after deprotonation, nitrogen of the azomethine group and pyrimidine nitrogen to give hexacoordinated tellurium(IV) complexes. The complexes have also been screened for their antimicrobial activities against various bacteria and fungi organisms.
\end{abstract}

Keywords: Salicylidene-trimethoprim Schiff base, Aryltellurium(IV), Diaryltellurium(IV), Antibacterial, Antifungal activities

\section{Introduction}

Trimethoprim and its derivatives are broad spectrum antimicrobial agents with anti-parasitic activity $^{1-6}$ and were first described by Roth and coworker ${ }^{5}$. Schiff bases, also known as azomethine due to presence of $-\mathrm{C}=\mathrm{N}$ - group, play important role in biological system, such as anticancer $^{7-12}$, antibacterial ${ }^{13-15}$, antiviral ${ }^{16-18}$, antifungal ${ }^{19-21}$ and other biological properties $^{22-27}$ and also have been extensively used as ligands in coordination chemistry because of their excellent donor abilities as chelating agent ${ }^{28-32}$. Medicinal chemists have reported new derivatives of trimethoprim(TMZ) ${ }^{33-35}$ including the Schiff base derived from salicylaldehyde $2-4,36,37$.

Also, aryltellurium(IV) chlorides are known ${ }^{38-54}$ to act as Lewis acids and form complexes with several N-, O- and S- donor bases. In view of this, we herein report some new complexes derived from aryltellurium(IV) trichlorides, $\mathrm{RTeCl}_{3}$ and diaryltellurium(IV) dichlorides, $\mathrm{R}_{2} \mathrm{TeCl}_{2}$ with salicylidene-trimethoprim Schiff base(Sal-TMP). 


\section{Experimental}

All preparations were carried out under dry $\mathrm{N}_{2}$ atmosphere and the solvents used were purified by standard method ${ }^{55,56}$ before use. The purity of compounds was checked by thin layer chromatography using silica gel-G (Merck). Melting points were determined in open capillary tube and are uncorrected.

Carbon, hydrogen and nitrogen analyses were obtained microanalytically from SAIF, Panjab University Chandigarh on a ThermoFinnigan CHNS analyser. Conductivity was measured in DMSO at $25 \pm 2{ }^{\circ} \mathrm{C}$ with dip type conductivity cell on a microprocessor based conductivity bridge type MICROSIL.

IR $\left(4000-400 \mathrm{~cm}^{-1}\right)$ and far IR $\left(400-50 \mathrm{~cm}^{-1}\right)$ spectra were recorded in $\mathrm{KBr} /$ polyethylene pellets on a FT-Infrared spectrophotometer model RZX (Perkin Elmer) at SAIF, Panjab University Chandigarh. Proton magnetic resonance spectra were recorded in DMSO- $\mathrm{d}_{6}$ using TMS as an internal reference on BRUKER AVANCE II 400 NMR spectrometer. The antimicrobial screening was carried out by tube dilution method at Department of Pharmaceutical Sciences, M. D. University, Rohtak, India.

\section{Preparation of aryltellurium(IV) trichlorides and diaryltellurium(IV) dichlorides}

p-Methoxyphenyltellurium(IV) $\quad$ trichloride $^{57,58}, \quad$ bis(p-methoxyphenyl)tellurium(IV) dichloride ${ }^{58,59}, \quad p$-ethoxyphenyltellurium (IV) trichloride ${ }^{60}$, bis( $p$-ethoxyphenyl)tellurium dichloride $^{60} p$-hydroxyphenyltellurium(IV) trichloride ${ }^{61}$, bis( $p$-hydroxyphenyl) tellurium(IV) dichloride $^{61}$, 3-methyl-4-hydroxyphenyltellurium(IV) trichloride $^{62}$ and bis(3-methyl-4hydroxyphenyl)tellurium(IV) dichloride ${ }^{62}$ were prepared by the reactions of $\mathrm{TeCl}_{4}$ with anisole, phenetole, phenol, $o$-cresol respectively, by the methods reported in the literature ${ }^{57-62}$.

\section{Preparation of salicylidene-trimethoprim Schiff base (Sal-TMP)}

Equimolar quantity of saturated methanolic solution of drug and salicylaldehyde were mixed thoroughly. To this mixture $0.1 \%$ methanolic $\mathrm{KOH}$ was added to adjust the $\mathrm{pH}$ of the solution between 7-8 and was refluxed for 2 hours. A clear yellowish-green coloured solution was obtained. After completion of the reaction, the Schiff base ligand was isolated by crystallization after volume reduction by evaporation. The crystalline product was filtered and dried under vacuum and kept in desiccator over $\mathrm{P}_{4} \mathrm{O}_{10}$ until further use. Yield $=$ $80 \%$, M.pt.(decomp.) $=188-190{ }^{\circ} \mathrm{C}$. Analysis (Calculated) $\mathrm{C}_{21} \mathrm{H}_{22} \mathrm{~N}_{4} \mathrm{O}_{4}: \mathrm{C}(63.95), \mathrm{H}(5.62)$ and $\mathrm{N}(14.20)$; Found: $\mathrm{C}(63.50), \mathrm{H}(5.27)$ and $\mathrm{N}(13.99)$.

\section{Preparation of salicylidene-trimethoprim complexes of aryltellurium(IV) trichlorides} and diaryltellurium(IV) dichlorides

Aryltellurium(IV) trichlorides, $\mathrm{ArTeCl}_{3}$ and diaryltellurium(IV) dichlorides $\mathrm{Ar}_{2} \mathrm{TeCl}_{2}$ (Ar= $p$-methoxyphenyl, $p$-ethoxyphenyl, $p$-hydroxyphenyl and 3-methyl-4-hydroxyphenyl), when reacted with sodium salt of salicylidene-trimethoprim in equimolar ratio, yield SalTMP.ArTeCl $\mathrm{Cl}_{2}$ and Sal-TMP.Ar $\mathrm{TeCl}_{2} \mathrm{Type}$ complexes.

Sodium salt of the ligand was prepared by reacting equimolar (1:1) quantity of sodium metal and Schiff base in methanol. The solvent was distilled off to obtain sodium salt of Schiff base. Then a methanolic saturated solution of $2 \mathrm{mmol}$ of aryltellurium(IV) trichloride or diaryltellurium(IV) dichloride was added dropwise to suspension of $2 \mathrm{mmol}$ of sodium salt of Schiff base in about $50 \mathrm{~mL}$ benzene under reflux. The reaction mixture was further refluxed for 3-4 hours, cooled and precipitated sodium chloride was filtered off. The filtrate was then concentrated to about one third of original volume under reduced pressure and cooled in an ice bath to obtain coloured product. This was filtered, washed with benzene + methanol (1:1) and dried in vacuum desiccator over $\mathrm{P}_{4} \mathrm{O}_{10}$. 


\section{Results and Discussion}

$\mathrm{TeCl}_{4}$ when heated with anisole ${ }^{57-59}$, phenetole ${ }^{60}$, phenol ${ }^{61}, o$-cresol ${ }^{62}$ (Ar-H) appears to undergo Friedel-Crafts type condensation reaction whereby $\mathrm{TeCl}_{3}{ }^{+}$unit attacks a position para to the methoxy/ethoxy/hydroxy groups in the aromatic rings, thus resulting in the formation of aryltellurium(IV) trichlorides and diaryltellurium(IV) dichlorides.

$$
\begin{gathered}
\mathrm{Ar}-\mathrm{H}+\mathrm{TeCl}_{4} \longrightarrow \mathrm{ArTeCl}_{3}+\mathrm{HCl} \\
2 \mathrm{Ar}-\mathrm{H}+\mathrm{TeCl}_{4} \longrightarrow \mathrm{Ar}_{2} \mathrm{TeCl}_{2}+2 \mathrm{HCl}
\end{gathered}
$$

Preparation of salicylidene-trimethoprim Schiff base (Sal-TMP) by the reaction of trimethoprim drug and salicylaldehyde can be represented by following equations.

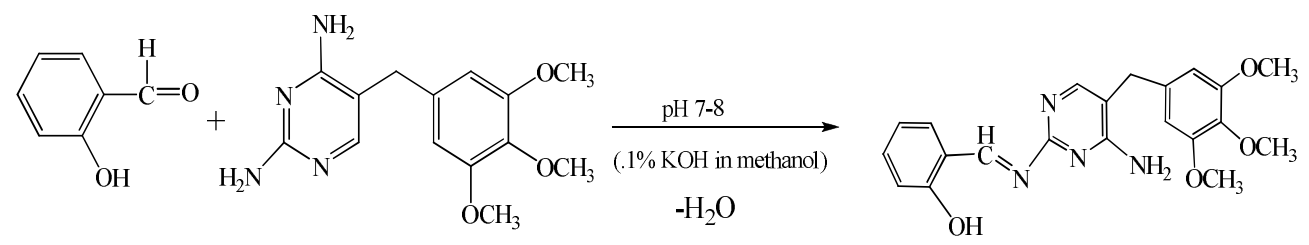

Sodium salt of salicylidene-trimethoprim Schiff base (Sal-TMP) reacts with aryltellurium(IV) trichlorides and diaryltellurium(IV) dichlorides in 1:1 molar ratio to yield the corresponding aryltellurium(IV) complexes.

$$
\begin{aligned}
& \mathrm{Sal}-\mathrm{TMP}+\mathrm{ArTeCl}_{3} \underset{\mathrm{NaCl}}{\stackrel{\mathrm{Na} / \mathrm{CH}_{3} \mathrm{OH}}{\longrightarrow}} \text { (Sal-TMP).ArTeCl } 2 \\
& \mathrm{Sal}-\mathrm{TMP}+\mathrm{Ar}_{2} \mathrm{TeCl}_{2} \underset{-\mathrm{NaCl}}{\stackrel{\mathrm{Na} / \mathrm{CH}_{3} \mathrm{OH}}{\longrightarrow}} \text { (Sal-TMP). } \mathrm{Ar}_{2} \mathrm{TeCl}_{2}
\end{aligned}
$$

All the tellurium(IV) complexes are coloured, crystalline solids, stable at room temperature and non-hygroscopic in nature. The complexes have been analysed for their tellurium, chlorine, carbon, hydrogen and nitrogen contents and the data along with their physical properties and yields are presented in Table 1.

\section{Conductance studies}

Molar conductance $\left(\Lambda_{M}\right)$ data for the complexes in DMSO are complied in Table 1. The $\Lambda_{M}$ value at $c a .10^{-3} \mathrm{M}$ for aryltellurium(IV) complexes in DMSO lie in the range 27.36-91.83 S $\mathrm{cm}^{2} \mathrm{~mol}^{-1}$ which predict the non electrolyte to $1: 1$ electrolyte $^{63,64}$ type behaviour of these complexes in DMSO, probably due to ionization into $\mathrm{ArTeCl}$.Sal- $\mathrm{TMP}^{+} / \mathrm{Ar}_{2} \mathrm{Te}$.Sal-TMP ${ }^{+}$ and $\mathrm{Cl}^{-}$in DMSO. This conductance behavoiur of tellurium(IV) salicylidene-trimethoprim Schiff base complexes is different from those of transition metal complexes ${ }^{65}$, which are reported to be non-electrolytes. The higher $\Lambda_{\mathrm{M}}$ values for some complexes may be due to steric factors and donor behaviour of DMSO to result in probable dissociation into solvated cation and $\mathrm{L}^{-}$along with $\mathrm{Cl}^{-}$in DMSO.

\section{Infrared spectra}

The IR data of Schiff base and its tellurium(IV) complexes are listed in Table 2. The spectra of Sal-TMP Schiff base complexes are quite complex and an attempt has therefore been made to identify the donor sites by comparing the spectra of complexes with parent ligand and $\mathrm{ArTeCl}_{3} / \mathrm{Ar}_{2} \mathrm{TeCl}_{2}$. 
Table 1. Analytical data, molar conductance and physical properties of salicylidene-trimethoprim Schiff base (Sal-TMP) complexes of tellurium(IV)

\begin{tabular}{|c|c|c|c|c|c|c|c|c|c|c|}
\hline \multirow[b]{2}{*}{$\begin{array}{l}\text { Compound } \\
\text { No. }\end{array}$} & \multirow[b]{2}{*}{$\begin{array}{l}\text { Complex } \\
\text { (Ar) }\end{array}$} & \multirow{2}{*}{$\begin{array}{c}\text { Empirical } \\
\text { formula } \\
\text { (Formula Wt.) }\end{array}$} & \multirow[b]{2}{*}{$\begin{array}{l}\text { Colour } \\
\text { (Yield, \%) }\end{array}$} & \multirow{2}{*}{$\begin{array}{l}\text { M. Pt. } \\
{ }^{\circ} \mathrm{C} \\
\text { dec. }\end{array}$} & \multicolumn{5}{|c|}{ Analyses \% Found (Calculated) } & \multirow{2}{*}{$\begin{array}{l}\Lambda_{\mathrm{M}} \text { at } c a \\
10^{-3} \mathrm{M} \mathrm{S} \\
\mathrm{cm}^{2} \mathrm{~mol}^{-1} \\
\text { in DMSO }\end{array}$} \\
\hline & & & & & $\mathrm{C}$ & $\mathrm{H}$ & $\mathrm{N}$ & $\mathrm{Te}$ & $\mathrm{Cl}$ & \\
\hline $\begin{array}{l}\text { Schiff } \\
\text { Base }\end{array}$ & Sal-TMP & $\mathrm{C}_{21} \mathrm{H}_{22} \mathrm{~N}_{4} \mathrm{O}_{4}$ & $\begin{array}{l}\text { Yellowish } \\
\text {-green }(80)\end{array}$ & $188-190$ & $\begin{array}{c}63.50 \\
(63.95)\end{array}$ & $\begin{array}{c}5.27 \\
(5.62)\end{array}$ & $\begin{array}{l}13.99 \\
(14.20)\end{array}$ & - & - & - \\
\hline I & $\begin{array}{l}\text { (Sal-TMP).ArTeCl }{ }_{2} \\
\text { (p-methoxyphenyl) }\end{array}$ & $\begin{array}{c}\mathrm{C}_{28} \mathrm{H}_{28} \mathrm{Cl}_{2} \mathrm{~N}_{4} \mathrm{O}_{5} \mathrm{Te} \\
(699.05)\end{array}$ & $\begin{array}{l}\text { Light cream } \\
(92)\end{array}$ & $230-232$ & $\begin{array}{c}47.88 \\
(48.11)\end{array}$ & $\begin{array}{c}3.99 \\
(4.04)\end{array}$ & $\begin{array}{c}7.75 \\
(8.01)\end{array}$ & $\begin{array}{c}18.07 \\
(18.24)\end{array}$ & $\begin{array}{c}9.89 \\
(10.01)\end{array}$ & 53.19 \\
\hline II & $\begin{array}{c}\text { (Sal-TMP).ArTeCl } \\
\text { (p-ethoxyphenyl) }\end{array}$ & $\begin{array}{c}\mathrm{C}_{29} \mathrm{H}_{30} \mathrm{Cl}_{2} \mathrm{~N}_{4} \mathrm{O}_{5} \mathrm{Te} \\
(713.08)\end{array}$ & $\begin{array}{c}\text { Cream } \\
(85)\end{array}$ & $258-260$ & $\begin{array}{c}48.12 \\
(48.85)\end{array}$ & $\begin{array}{c}3.87 \\
(4.24)\end{array}$ & $\begin{array}{l}7.52 \\
(7.86)\end{array}$ & $\begin{array}{c}17.38 \\
(17.89)\end{array}$ & $\begin{array}{c}9.52 \\
(9.81)\end{array}$ & 52.88 \\
\hline III & $\begin{array}{c}(\mathrm{Sal}-\mathrm{TMP}) \cdot \mathrm{ArTeCl}_{2} \\
(p \text {-hydroxyphenyl })\end{array}$ & $\begin{array}{c}\mathrm{C}_{27} \mathrm{H}_{26} \mathrm{Cl}_{2} \mathrm{~N}_{4} \mathrm{O}_{5} \mathrm{Te} \\
(685.03)\end{array}$ & $\begin{array}{l}\text { Dark cream } \\
\quad(94)\end{array}$ & $208-210$ & $\begin{array}{c}46.78 \\
(47.34)\end{array}$ & $\begin{array}{c}3.50 \\
(3.83)\end{array}$ & $\begin{array}{c}7.88 \\
(8.18)\end{array}$ & $\begin{array}{c}18.28 \\
(18.63)\end{array}$ & $\begin{array}{c}9.98 \\
(10.21)\end{array}$ & 55.73 \\
\hline IV & $\begin{array}{c}\text { (Sal-TMP).ArTeCl } \\
\text { (3-methyl-4- } \\
\text { hydroxyphenyl) }\end{array}$ & $\begin{array}{c}\mathrm{C}_{28} \mathrm{H}_{28} \mathrm{Cl}_{2} \mathrm{~N}_{4} \mathrm{O}_{5} \mathrm{Te} \\
(699.05)\end{array}$ & $\begin{array}{l}\text { Light } \\
\text { cream } \\
(80)\end{array}$ & $198-200$ & $\begin{array}{c}47.58 \\
(48.11)\end{array}$ & $\begin{array}{c}3.84 \\
(4.04)\end{array}$ & $\begin{array}{c}7.80 \\
(8.01)\end{array}$ & $\begin{array}{c}18.15 \\
(18.24)\end{array}$ & $\begin{array}{c}9.85 \\
(10.01)\end{array}$ & 58.68 \\
\hline $\mathrm{V}$ & $\begin{array}{c}(\mathrm{Sal}-\mathrm{TMP}) \cdot \mathrm{Ar}_{2} \mathrm{TeCl} \\
(p \text {-methoxyphenyl })\end{array}$ & $\begin{array}{c}\mathrm{C}_{35} \mathrm{H}_{35} \mathrm{ClN}_{4} \mathrm{O}_{6} \mathrm{Te} \\
(770.73)\end{array}$ & $\begin{array}{c}\text { Pale yellow } \\
\text { (84) }\end{array}$ & $130-132$ & $\begin{array}{c}53.84 \\
(54.54)\end{array}$ & $\begin{array}{c}4.31 \\
(4.58)\end{array}$ & $\begin{array}{c}7.11 \\
(7.27)\end{array}$ & $\begin{array}{c}16.43 \\
(16.56)\end{array}$ & $\begin{array}{c}4.42 \\
(4.60)\end{array}$ & 91.83 \\
\hline VI & $\begin{array}{c}\text { (Sal-TMP).Ar }{ }_{2} \mathrm{TeCl} \\
(p \text {-ethoxyphenyl) }\end{array}$ & $\begin{array}{c}\mathrm{C}_{37} \mathrm{H}_{39} \mathrm{ClN}_{4} \mathrm{O}_{6} \mathrm{Te} \\
(798.78)\end{array}$ & $\begin{array}{l}\text { Light yellow } \\
\text { (86) }\end{array}$ & $150-152$ & $\begin{array}{c}54.84 \\
(55.63)\end{array}$ & $\begin{array}{c}4.53 \\
(4.92)\end{array}$ & $\begin{array}{c}6.84 \\
(7.01)\end{array}$ & $\begin{array}{c}15.50 \\
(15.97)\end{array}$ & $\begin{array}{c}4.30 \\
(4.44)\end{array}$ & 35.90 \\
\hline VII & $\begin{array}{l}\text { (Sal-TMP).Ar } \mathrm{Ar}_{2} \mathrm{TeCl} \\
(p \text {-hydroxyphenyl) }\end{array}$ & $\begin{array}{c}\mathrm{C}_{33} \mathrm{H}_{31} \mathrm{ClN}_{4} \mathrm{O}_{6} \mathrm{Te} \\
(742.68)\end{array}$ & $\begin{array}{l}\text { Red } \\
(89)\end{array}$ & $146-148$ & $\begin{array}{c}52.84 \\
(53.37)\end{array}$ & $\begin{array}{c}4.01 \\
(4.21)\end{array}$ & $\begin{array}{c}7.27 \\
(7.54)\end{array}$ & $\begin{array}{c}16.89 \\
(17.18)\end{array}$ & $\begin{array}{c}4.52 \\
(4.77)\end{array}$ & 36.00 \\
\hline VIII & $\begin{array}{c}\text { (Sal-TMP). } \mathrm{Ar}_{2} \mathrm{TeCl} \\
\text { (3-methyl-4- } \\
\text { hydroxyphenyl) }\end{array}$ & $\begin{array}{c}\mathrm{C}_{35} \mathrm{H}_{35} \mathrm{ClN}_{4} \mathrm{O}_{6} \mathrm{Te} \\
(770.73)\end{array}$ & $\begin{array}{l}\text { Brown } \\
(78)\end{array}$ & $140-142$ & $\begin{array}{l}53.80 \\
(54.54)\end{array}$ & $\begin{array}{c}4.34 \\
(4.58)\end{array}$ & $\begin{array}{c}7.09 \\
(7.27)\end{array}$ & $\begin{array}{c}16.45 \\
(16.56)\end{array}$ & $\begin{array}{c}4.32 \\
(4.60)\end{array}$ & 27.36 \\
\hline
\end{tabular}


Table 2. Important infrared absorption bands $\left(\mathrm{cm}^{-1}\right)$ of Schiff base (Sal-TMP) and complexes

\begin{tabular}{|c|c|c|c|c|c|c|c|}
\hline Compound & $v_{(O-}$ & $v_{(1}$ & $v_{(C}$ & $v_{(C}$ & $v_{(\mathrm{C}-}$ & $v_{(\mathrm{Te}-\mathrm{N})}$ & $v_{(\mathrm{Te}}$ \\
\hline Sal-TMP & $2836 \mathrm{w}$ & $3317 \mathrm{~m} \mathrm{3119} \mathrm{m}$ & $1636 \mathrm{sh}$ & $1633 \mathrm{w} 1593 \mathrm{~s}$ & $1263 \mathrm{~s}$ & - & - \\
\hline $\bar{I}$ & - & $3323 \mathrm{~m} 3184 \mathrm{~m}$ & $1674 \mathrm{mb}$ & $1644 \mathrm{mb} 1587 \mathrm{~s}$ & $1341 \mathrm{~s}$ & $415 \mathrm{~m}$ & $288 \mathrm{w}$ \\
\hline II & - & $3323 \mathrm{~m} 3170 \mathrm{~m}$ & $1647 \mathrm{sh}$ & **1586 s & $1304 \mathrm{~s}$ & $420 \mathrm{~m}$ & $295 w$ \\
\hline III & 3405 m* & $3323 \mathrm{~m} 3150 \mathrm{~m}$ & $1674 \mathrm{mb}$ & $1641 \mathrm{mb} 1586 \mathrm{~s}$ & $1341 \mathrm{~s}$ & $419 \mathrm{~s}$ & $270 \mathrm{w}$ \\
\hline IV & 3398 m* & $3319 \mathrm{~m} 3172 \mathrm{~m}$ & $1652 \mathrm{mb}$ & 1649 & $1333 \mathrm{~s}$ & $450 \mathrm{~s}$ & $277 \mathrm{w}$ \\
\hline V & - & $3325 \mathrm{~m}$ & 164 & $* * 1$ & $1333 \mathrm{~s}$ & $416 \mathrm{~s}$ & $290 \mathrm{w}$ \\
\hline VI & - & $3320 \mathrm{~m} 3158 \mathrm{~m}$ & $1640 \mathrm{sh}$ & $* * 1590 \mathrm{~s}$ & $1331 \mathrm{~s}$ & $410 \mathrm{~m}$ & $273 w$ \\
\hline VII & $3401 \mathrm{w}^{*}$ & $3324 \mathrm{~m} 3168 \mathrm{~m}$ & $1674 \mathrm{mb}$ & $1643 \mathrm{mb} 1584 \mathrm{~s}$ & $1340 \mathrm{~s}$ & $418 \mathrm{~m}$ & $285 \mathrm{w}$ \\
\hline VIII & $3463 \mathrm{w}^{*}$ & $3304 \mathrm{~m} 3106 \mathrm{~m}$ & $1634 \mathrm{sh}$ & $1643 \mathrm{mb} 1584 \mathrm{~s}$ & $1340 \mathrm{~s}$ & $422 \mathrm{~m}$ & $287 \mathrm{w}$ \\
\hline
\end{tabular}

$s=$ sharp, m=medium, mb=medium broad, sh=shoulder, $w=w e a k$, "Due to phenolic $\mathrm{OH}$ of Rte and $\mathrm{R}_{2} \mathrm{Te}$ moieties; ${ }^{* *}$ band not resolved due to overlapping of band $\boldsymbol{v}_{(C=N)}$

Examination of the Schiff base (Sal-TMP) spectrum shows the presence of the hydrogen bonded conjugated- chelate ring system ${ }^{66-68}$ with its centre at $\sim 2832 \mathrm{~cm}^{-1}$. Thus intramolecular $\mathrm{H}$ - bonding is occurring by means of the formation of a quasi six- membered ring involving the $\mathrm{OH}----\mathrm{N}=\mathrm{C}$ bond.<smiles>C1=Cc2ccccc2O[NH+]=1</smiles>

Figure 1. Hydrogen bonding

Thus band disappear on chelation with aryltellurium(IV) chlorides ${ }^{69}$. Hydrogen bond contributes to planarity of the molecule which helps in chelation ${ }^{70}$. Also an intense ligand band at $1263 \mathrm{~cm}^{-1}$ (phenolic -C-O) in free ligand has shifted to higher frequency side in complexes. All these indicate that the hydroxyl group of salicylaldehyde of Schiff base is involved in coordination with tellurium ${ }^{71-73}$.

In addition, the spectra of the Schiff base shown shoulder at $1636 \mathrm{~cm}^{-1}$ with slightly resolved weak band at $1633 \mathrm{~cm}^{-1}$ and sharp band at $1593 \mathrm{~cm}^{-1}$ assigned to $v_{(\mathrm{C}=\mathrm{N})}$ mode for vibration of azomethine group ${ }^{2,4,65,74-76}$ and $v_{(\mathrm{C}=\mathrm{N}) \text { pyrim. For pyrimidine ring }}{ }^{4,77}$. These shift in aryltellurium Schiff base complexes towards higher and lower value ${ }^{74-79}$ reflecting that ligand coordinate through nitrogen atom of azomethine and pyrimidine ring.

The medium intensity band at $3317 \mathrm{~cm}^{-1}$ and $3119 \mathrm{~cm}^{-1}$ due to $v_{(\mathrm{N}-\mathrm{H})}$ asymmetric and symmetric vibrations respectively indicate the non involvement of the nitrogen atom of $\mathrm{NH}_{2}$ group attached to pyrimidine in coordination. The appearance of new weak bands around $270-295 \mathrm{~cm}^{-1}$ due to $v_{(\mathrm{Te}-\mathrm{O})}^{80-83}$ mode and medium to strong band in the range of 410-422 $\mathrm{cm}^{-1}$ due to $v_{(\mathrm{Te}-\mathrm{N})}$ mode ${ }^{84}$ further supports the involvement of phenolic oxygen (after deprotonation), azomethine and pyrimidine nitrogen atoms of Schiff base in the coordination.

Thus, IR data predict the tridentate nature of Sal-TMP involving azomethine nitrogen atom, phenolic oxygen after deprotonation and pyrimidine ring nitrogen giving rise to twosix and four membered chelate rings with the tellurium centre. 


\section{${ }^{1}$ H NMR spectra}

In order to identify the solution structure of Schiff base (Sal-TMP) and its complexes, ${ }^{1} \mathrm{H}$ NMR spectra were recorded in DMSO- $\mathrm{d}_{6}$ and given in Table 3. The proton resonance of the $\mathrm{OH}$ group at $10.92 \delta \mathrm{ppm}^{76,85}$ in Schiff base due to presence of intramolecular hydrogen bonding ${ }^{74}$ disappear on complexation indicating the involvement of phenolic oxygen in the coordination via deprotonation ${ }^{76}$. The azomethine protons which resonate as a singlet at $10.02 \delta \mathrm{ppm}$, the coordination of azomethine nitrogen to tellurium in the complexes are clearly demonstrated by a downfield shift of the peak ${ }^{86}$.

Table 3. ${ }^{1} \mathrm{H}$ NMR spectral data of Schiff base (Sal-TMP) and complexes in DMSO- $\mathrm{d}_{6}$

\begin{tabular}{|c|c|}
\hline Compound & Chemical Shift, $\delta$ ppm \\
\hline Sal-TMP & $\begin{array}{l}2.525(\mathrm{~s}, 2 \mathrm{H}, \text { methylene }), \quad 3.551-3.745\left(\mathrm{~s}, \quad 9 \mathrm{H},-\mathrm{OCH}_{3}\right), \quad 5.672(\mathrm{~s}, 1 \mathrm{H}, \\
\text { pyrimidine }), 6.508\left(\mathrm{~s}, 2 \mathrm{H},-\mathrm{NH}_{2}\right), 7.526-8.169(\mathrm{~m}, 6 \mathrm{H}, \text { aromatic proton), } \\
10.021(\mathrm{~s}, 1 \mathrm{H}, \text { azomethine }), 10.921(\mathrm{~s}, 1 \mathrm{H}, \mathrm{Schiff} \text { base } \mathrm{OH})\end{array}$ \\
\hline I & $\begin{array}{l}2.539(\mathrm{~s}, 2 \mathrm{H}, \text { methylene }), 3.598-3.840\left(\mathrm{~s}, 12 \mathrm{H},-\mathrm{OCH}_{3}\right), 6.574(\mathrm{~s}, 1 \mathrm{H}, \\
\text { pyrimidine }), 6.926\left(\mathrm{~s}, 2 \mathrm{H},-\mathrm{NH}_{2}\right), 7.383-7.522(\mathrm{~m}, 10 \mathrm{H}, \text { aromatic proton }), \\
10.209(\mathrm{~s}, 1 \mathrm{H}, \text { azomethine })\end{array}$ \\
\hline II & $\begin{array}{l}1.335\left(\mathrm{t}, 3 \mathrm{H},-\mathrm{OCH}_{2} \mathrm{CH}_{3}\right), 2.546(\mathrm{~s}, 2 \mathrm{H}, \text { methylene }), 3.963\left(\mathrm{q}, 2 \mathrm{H},-\mathrm{OCH}_{2} \mathrm{CH}_{3}\right) \text {, } \\
3.515-3.875\left(\mathrm{~s}, 9 \mathrm{H},-\mathrm{OCH}_{3}\right), 6.540(\mathrm{~s}, 1 \mathrm{H}, \text { pyrimidine }), 6.950\left(\mathrm{~s}, 2 \mathrm{H},-\mathrm{NH}_{2}\right), \\
6.993-7.809(\mathrm{~m}, 10 \mathrm{H} \text {, aromatic proton), } 10.193(\mathrm{~s}, 1 \mathrm{H} \text {, azomethine })\end{array}$ \\
\hline III & $\begin{array}{l}2.548(\mathrm{~s}, 2 \mathrm{H}, \quad \text { methylene }), \quad 3.601-3.783\left(\mathrm{~s}, 9 \mathrm{H},-\mathrm{OCH}_{3}\right), \quad 6.569(\mathrm{~s}, 1 \mathrm{H}, \\
\text { pyrimidine }), 6.914\left(\mathrm{~s}, 2 \mathrm{H},-\mathrm{NH}_{2}\right), 7.370-7.787(\mathrm{~m}, 10 \mathrm{H}, \text { aromatic proton }), \\
8.155(\mathrm{~s}, 1 \mathrm{H}, \text { phenolic } \mathrm{OH} \text { of } \mathrm{RTe}), 10.201(\mathrm{~s}, 1 \mathrm{H}, \text { azomethine })\end{array}$ \\
\hline IV & $\begin{array}{l}2.536(\mathrm{~s}, 2 \mathrm{H}, \text { methylene }), 2.527\left(\mathrm{~s}, 3 \mathrm{H},-\mathrm{CH}_{3}\right), 3.587-3.770\left(\mathrm{~s}, 9 \mathrm{H},-\mathrm{OCH}_{3}\right) \text {, } \\
\text { 6.605(s, } 1 \mathrm{H}, \text { pyrimidine }), 6.918\left(\mathrm{~s}, 2 \mathrm{H},-\mathrm{NH}_{2}\right), 7.051-7.671(\mathrm{~m}, 9 \mathrm{H} \text {, aromatic } \\
\text { proton }), 8.251(\mathrm{~s}, 1 \mathrm{H} \text {, phenolic } \mathrm{OH} \text { of } \mathrm{RTe}), 10.239(\mathrm{~s}, 1 \mathrm{H} \text {, azomethine })\end{array}$ \\
\hline V & $\begin{array}{l}2.528(\mathrm{~s}, 2 \mathrm{H}, \text { methylene }), 3.673-3.877\left(\mathrm{~s}, 15 \mathrm{H},-\mathrm{OCH}_{3}\right), 6.516(\mathrm{~s}, 1 \mathrm{H} \text {, } \\
\text { pyrimidine }), 6.882\left(\mathrm{~s}, 2 \mathrm{H},-\mathrm{NH}_{2}\right), 6.910-7.804(\mathrm{~m}, 14 \mathrm{H}, \text { aromatic proton }) \\
10.186(\mathrm{~s}, 1 \mathrm{H}, \text { azomethine })\end{array}$ \\
\hline VI & $\begin{array}{l}\left.\text { 1.363(t, } 6 \mathrm{H},-\mathrm{OCH}_{2} \mathbf{C H}_{3}\right), 2.531(\mathrm{~s}, 2 \mathrm{H}, \text { methylene }), 4.019\left(\mathrm{q}, 4 \mathrm{H},-\mathrm{OCH}_{2} \mathrm{CH}_{3}\right) \text {, } \\
3.555-3.765\left(\mathrm{~s}, 9 \mathrm{H},-\mathrm{OCH}_{3}\right), 6.510(\mathrm{~s}, 1 \mathrm{H}, \text { pyrimidine }), 6.924\left(\mathrm{~s}, 2 \mathrm{H},-\mathrm{NH}_{2}\right) \text {, } \\
\text { 7.524-7.805(m, } 14 \mathrm{H} \text {, aromatic proton }), 10.216(\mathrm{~s}, 1 \mathrm{H} \text {, azomethine })\end{array}$ \\
\hline VII & $\begin{array}{l}2.545(\mathrm{~s}, 2 \mathrm{H}, \quad \text { methylene }), 3.568-3.926\left(\mathrm{~s}, 9 \mathrm{H},-\mathrm{OCH}_{3}\right), 6.554(\mathrm{~s}, 1 \mathrm{H} \text {, } \\
\text { pyrimidine }), 6.884\left(\mathrm{~s}, 2 \mathrm{H},-\mathrm{NH}_{2}\right), 7.488-7.678(\mathrm{~m}, 14 \mathrm{H}, \text { aromatic proton), } \\
8.249\left(\mathrm{~s}, 2 \mathrm{H}, \text { phenolic } \mathrm{OH} \text { of } \mathrm{R}_{2} \mathrm{Te}\right), 10.238(\mathrm{~s}, 1 \mathrm{H}, \text { azomethine })\end{array}$ \\
\hline VIII & $\begin{array}{l}2.538(\mathrm{~s}, 2 \mathrm{H}, \text { methylene }), 2.529\left(\mathrm{~s}, 6 \mathrm{H},-\mathrm{CH}_{3}\right), 3.555-3.750\left(\mathrm{~s}, 9 \mathrm{H},-\mathrm{OCH}_{3}\right) \text {, } \\
6.502(\mathrm{~s}, 1 \mathrm{H}, \text { pyrimidine }), 6.926\left(\mathrm{~s}, 2 \mathrm{H},-\mathrm{NH}_{2}\right), 7.126-7.868(\mathrm{~m}, 12 \mathrm{H} \text {, aromatic } \\
\text { proton }), 8.149\left(\mathrm{~s}, 2 \mathrm{H} \text {, phenolic } \mathrm{OH} \text { of } \mathrm{R}_{2} \mathrm{Te}\right), 10.239(\mathrm{~s}, 1 \mathrm{H} \text {, azomethine })\end{array}$ \\
\hline
\end{tabular}
$s=$ singlet,$q=$ quartet,$t=$ triplet, $m=$ multiplet

The characterstic downfield shifting of proton signal in all complexes observed in region $5.67 \delta \mathrm{ppm}$ is due to pyrimidine proton in Schiff base clearly indicate the coordination through pyrimidine nitrogen atom ${ }^{87}$. The signal due to $-\mathrm{NH}$ proton is observed around $6.51 \delta \mathrm{ppm}$ which remain intact with slight variation in complexes is due to the proton bounded to nitrogen experience quadruple effect ${ }^{87}$. Thus salicylidene-trimethoprim act as a tridentate $-\mathrm{N},-\mathrm{N},-\mathrm{O}$ chelating ligand in Sal-TMP.ArTeCl $\mathrm{C}_{2}$ and Sal-TMP. $\mathrm{Ar}_{2} \mathrm{TeCl}$ complexes giving six coordinate tellurium having distorted octahedral geometry in these complexes as predicated from IR studies as well. The proposed structures are as given below (Figure 2). 


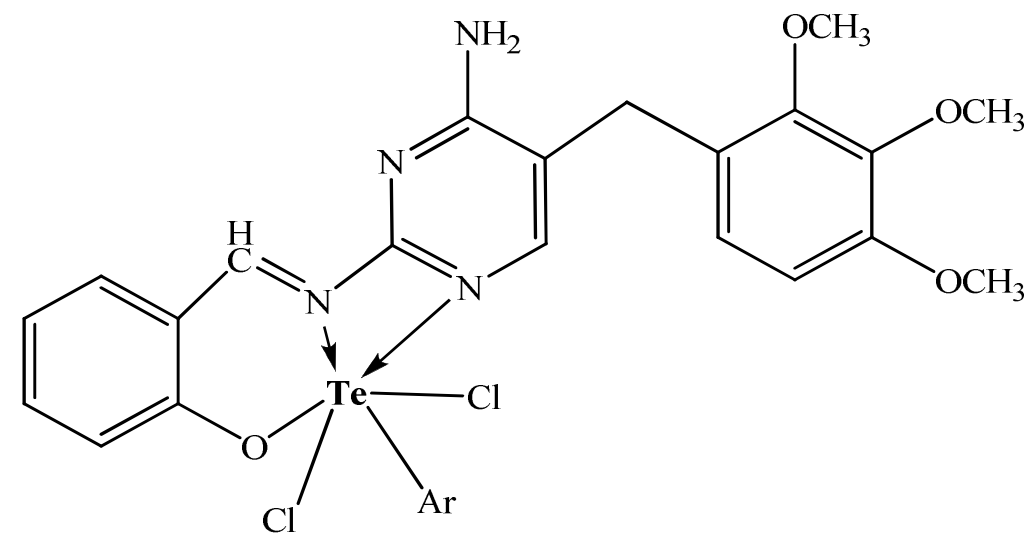<smiles></smiles>

Sal-TMP.Ar ${ }_{2} \mathrm{TeCl}$

$\mathrm{Ar}=p$-methoxyphenyl, $p$-ethoxyphenyl, $p$-hydroxyphenyl and 3-methyl-4-hydroxyphenyl

Figure 2. Proposed structures of complexes

\section{Biological Activity}

The salicylidene-trimethoprim Schiff base (Sal-TMP) and newly synthesized aryltellurium(IV) schiff base complexes were evaluated for their antimicrobial activity in vitro against Gram +ve bacteria (S. aureus ATCC 11632 and B. cereus MTCC 7350), Gram -ve bacteria (E. coli ATCC 35218, P. aeruginosa ATCC 23564, S. typhi ATCC 15499 and $P$. rettgeri DRDE) and fungal strains (A. niger, A. fumigates and A. flavus) by tube dilution method $^{88}$. Dilution of test and standard compounds were prepared double strength nutrient broth- I.P (Antibacterial) and Sabouraud Dextrose Broth -I.P (Antifungal) ${ }^{89}$. The samples were incubated at $37 \pm 1^{\circ} \mathrm{C}$ for $24 \mathrm{~h}$ (bacteria), $25 \pm 1^{\circ} \mathrm{C}$ for 7 days (A. niger), $30 \pm 1^{\circ} \mathrm{C}$ for 15 days (A. flavus), $35 \pm 1{ }^{\circ} \mathrm{C}$ for $72 \mathrm{~h}$ (A. fumigates) respectively and results were recorded in terms of MIC (The lowest concentration of test substances which inhibited values are presented in the Table 4.

The data show that the Sal-TMP complexes of aryltellurium(IV) exhibit more antimicrobial activity towards bacteria as compared to fungii. The antibacterial activity shows following trend. 
Sal-TMP.Ar $\mathrm{ArCl}_{2} \mathrm{Te}$ Sal-TMP.ArTeCl $\mathrm{Cl}_{2} \approx$ Sal-TMP Schiff base

Sal-TMP. $\mathrm{Ar}_{2} \mathrm{TeCl}$ and Sal-TMP.ArTeCl $\mathrm{Cl}_{2}$ complexes have activity towards $S$. typhi and more effectively against $B$. cereus but Schiff base does not show activity against these bacterial strains. Schiff base and its complexes show almost similar activity against fungal strains.

Table 4. Minimum Inhibitory Concentration, MIC, $\mu \mathrm{g} / \mathrm{mL}$; (-) Resistant

\begin{tabular}{|c|c|c|c|c|c|c|c|c|c|}
\hline \multirow[b]{2}{*}{ 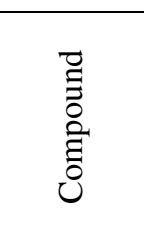 } & \multicolumn{6}{|c|}{ Bacteria strains } & \multicolumn{3}{|c|}{ Fungal strains } \\
\hline & $\begin{array}{c}S . \\
\text { aureus } \\
\text { (ATCC } \\
11632 \text { ) }\end{array}$ & $\begin{array}{l}\text { S. typhi } \\
\text { (ATCC } \\
15499 \text { ) }\end{array}$ & $\begin{array}{c}P . \\
\text { aeruginosa } \\
\text { (ATCC } \\
\text { 23564) }\end{array}$ & $\begin{array}{c}E . \\
a \quad c o l i \\
(\mathrm{ATCC} \\
35218)\end{array}$ & $\begin{array}{c}\text { B. cereus } \\
\text { (MTCC } \\
7350)\end{array}$ & $\begin{array}{c}P . \\
\text { rettgeri } \\
\text { (DRDE } \\
\text { strain) }\end{array}$ & A. niger & $\begin{array}{c}A . \\
\text { fumigates }\end{array}$ & $\begin{array}{l}\text { A. } \\
\text { flavus }\end{array}$ \\
\hline Sal-TMP & 2.5 & - & 1.25 & 5.0 & - & - & 20 & 5.0 & 1.25 \\
\hline I & 2.5 & - & 1.25 & - & 0.625 & 5.0 & - & - & - \\
\hline II & 5.0 & - & 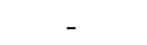 & - & 1.25 & 2.5 & 20 & 5.0 & 1.25 \\
\hline III & - & 20 & 10 & 5.0 & 1.25 & - & 5.0 & - & - \\
\hline IV & 1.25 & 2.5 & 1.25 & 5.0 & - & - & 5.0 & 10 & 5.0 \\
\hline V & - & 20 & 10 & 5.0 & 1.25 & - & 20 & - & - \\
\hline VI & 1.25 & 2.5 & 1.25 & 5.0 & - & - & - & 5.0 & - \\
\hline VII & 1.25 & - & 5.0 & 1.25 & 0.625 & 5.0 & - & 10 & - \\
\hline VIII & 2.5 & - & 1.25 & 5.0 & - & - & 20 & 5.0 & 1.25 \\
\hline
\end{tabular}

\section{Conclusion}

Aryltellurium(IV) and diaryltellurium(IV) dichlorides upon reaction with Schiff base(Sal-TMP) derived from salicylaldehyde and trimethoprim yield new complexes of tellurium(IV). The synthesized complexes were characterized by elemental analyses, conductance measurement, IR and ${ }^{1} \mathrm{H}$ NMR spectral studies. The analytical data suggest that the Sal-TMP Schiff base complexes have 1:1 stoichiometry. The Schiff bases (Sal-TMP) in these complexes functions as a uninegative tridentate ligand through azomethine nitrogen, phenolic oxygen after deprotonation and pyrimidine ring nitrogen atoms. Based on these studies, distorted octahedral geometry with two chelating rings has been assigned to these complexes. The complexes have been observed to possess substantial antimicrobial activity especially against bacteria.

\section{Acknowledgement}

The authors are grateful to M. D. University, Rohtak for providing the necessary facilities. One of the authors (Deepak) is also thankful to UGC New Delhi for providing fellowship. We also thank SAIF, Panjab University Chandigarh for providing the CHN analyses and spectral data.

\section{References}

1. Burchall J J, Corcoran J W and Hahn F E, Eds., Antibiotics, Springer, New York, NY, USA, $1975,3$.

2. Iqbal M S, Khan A H, Loothar B A and Bukhari I H, Med Chem Rev., 2009, 18, 31-42.

3. Blower P J, Аnnu Rep Prog Chem Sect A, 1999, 95, 631-655; DOI:10.1039/A808038K

4. Dhahir S A, Al-Sahib S A, Al Razaq W A and Kadhim N J, Baghdad Sci J., 2010, 7(1), 621.

5. Dr. Karl Thomae Gmb H, (Biberach an der Riss. DE), United States Patent. 4829058.

6. EL-Mossalamy E H, AL-Thabati S A, ALNomaiser F M, Commun Fac Sci Univ Ank Series B, 2005, 51(2), 21-30 
7. Sinha D, Tiwari A K, Singh S, Shukla G, Mishra P, Chandra H and Mishra A K, Eur J Med Chem., 2008, 43(1), 160-165; DOI:10.1016/j.ejmech.2007.03.022

8. Crowe A J, Smith P J and Atassi G, Chem Biol Interact., 1980, 32(1-2), 171-178; DOI:10.1016/0009-2797(80)90075-7

9. Wang M, Wang L F, Li Y Z, Li Q X, Xu Z D and Qu D M, Trans Met Chem., 2001, 26(3), 307-310; DOI:10.1023/A:1007159301849

10. Zishen Wu, Ziqi Gui and Zhenhuan Yen, Synth React Inorg Met Org Chem., 1990, 20(3), 335-344.

11. Desai S B, Desai P B and Desai K R, Hetrocycl Commun., 2001, 7(1), 83-90; DOI:10.1515/HC.2001.7.1.83

12. Dhumwad S D, Gudasi K B and Goudar T R, Indian J Chem., 1994, 33A, 320-324.

13. Przybylski P, Huczynski A, Pyta K, Brzezinski B and Bartl F, Curr Org Chem., 2009, 13(2), 124-148; DOI:10.2174/138527209787193774

14. Pandeya S N, Sriram D, Nath G and De Clercq E, Pharm Acta Hely., 1999, 74(1), 7-11.

15. Karthikeyan M S, Parsad D J, Poojary B, Bhat K S, Holla B S and Kumari N S, Bioorg. Med. Chem., 2006, 14(22), 7482-7489; DOI:10.1016/j.bmc.2006.07.015

16. Holla B S, Akberali P M and Shivananda M K, II Farmaco, 2001, 56(12), 919-927; DOI:10.1016/S0014-827X(01)01124-7

17. Jarrahpour A, Khalili D, De Clercq E, Salmi C and Brunel J M, Molecules, 2007, 12(8), 1720-1730; DOI:10.3390/12081720

18. Da Silva C M, da Silva D L, Modolo L V, Alves R B, de Resende, M A, Martins C V B and de Fatima A J, Adv Res., 2011, 2, 1-8; DOI:10.1016/j.jare.2010.05.004

19. Singh H, Yadav L D S and Mishra S B S, J Inorg Nucl Chem., 1981, 43(7), 17011704; DOI:10.1016/0022-1902(81)80367-3

20. Saravanan G, Pannerselvam P and Prakash C R, J Adv Pharm Techn Res., 2010, 1(3), 320-325; DOI:10.4103/0110-5558.72426

21. Panneerselvam P, Nair R R, Vijayalakshmi G, Subramanian E H and Sridhar S K, Eur. J. Med. Chem. 2005, 40, 225-229; DOI:10.1016/j.ejmech.2004.09.003

22. Singh N K and Singh S B, Indian J Chem., 2001, 40A, 1070-1075.

23. Walsh O M, Meegan M J, Prendergast R M, Nakib T A, Eur J Med Chem., 1996, 31, 989-1000; DOI:10.1016/S0223-5234(97)86178-8

24. Odabasoglu M, Arslan F, Ölmez H and Büyükgüngör O, Dyes and Pigments, 2007, 75(3), 507-515; DOI:10.1016/j.dyepig.2006.06.033

25. Vicini P, Geronikaki A, Incerti M, Busonera B, Poni G, Cabras C A and Colla P L, Bioorg Med Chem., 2003, 11(23), 4785-4789; DOI:10.1016/S09680896(03)00493-0

26. Pandeya S N, Sriram D, Nath G and DeClercq E, Eur J Pharm Sci., 1999, 9, 25-31.

27. Samadhiya S and Halve A, Orient J Chem., 2001, 17, 119-122.

28. Celik Ö, Ulusoy M, Tas E and Ide S, Anal Sciences, 2007, 23, x185.

29. Mahmoud M R, El-Gyar S A, Mousrafa A A and Shaker A, Polyhedron, 1987, 6(5), 1017-1020; DOI:10.1016/S0277-5387(00)80947-X

30. Qing-Yu H, Zheng-Hua M and Ya-Me Z, J Coord Chem., 1990, 21(3), 199-207; DOI:10.1080/00958979009409716

31. Cozzi P G, Chem Soc Rev., 2004, 33, 410-421; DOI:10.1039/B307853C

32. Tarafder M T H, Jin KT, Crouse K A, Ali A M. Yamin, B $M$ and Fun H K, Polyhedron, 2002, 21(25-26), 2547-2554; DOI:10.1016/S0277-5387(02)01188-9

33. Pandeya S N, Sriram D, Clercq E DE., Pannecouque C and Witvrouw M, Indian J Pharm Sci., 1998, 60(4), 207-212. 
34. Fleischman S H and Brooks C L, Proteins: Structure, Function and Genetics, 1990, 7, 52-61.

35. Suling W J, Reynolds R C, Barrow E W, Wilson L N, Piper J R and Barrow W W, J Antimicrobial Chemother., 1998, 42(6), 811-815; DOI:10.1093/jac/42.6.811

36. Gulcan M, Sonmez M and Berber I, Turkey J Chem., 2012, 36(1), 189-200.

37. Raman N, Kulandaisamy A and Jeyasubramanian K, Synth React Inorg Met Org Chem., 2001, 31(7), 1249-1270.

38. Wynne K J and Pearson P S, Inorg Chem., 1971, 10(12), 2735-2739; DOI:10.1021/ic50106a022

39. Wynne K J and Pearson P S, J Chem Soc Commun., 1970, 556-557; DOI:10.1039/C2970000556B

40. Wynne K J, Clark A J and Berg M, J Chem Soc Dalton Trans., 1972, 2370-2374; DOI:10.1039/DT9720002370

41. Clark E R, Collet A J and Naik D G, J Chem Soc Dalton Trans., 1973, 1961-1962; DOI:10.1039/DT9730001961

42. Berg M C, Diss Abstr Int., 1972, 33, 2982.

43. Srivastava T N, Singh M and Singh H B, Indian J Chem., 1982, 21A, 307-309.

44. Srivastava T N, Srivastava R C and Srivastava M, Indian J Chem., 1982, 21A, 539.

45. Srivastava T N, Srivastava R C and Srivastava V K, J Indian Chem Soc., 1983, 60, 891-892.

46. Garad M V, Polyhedron, 1985, 4(8), 1353-1355; DOI:10.1016/S0277-5387(00)86963-6

47. Verma K K and Reena, Synth React Inorg Met-Org Chem., 1999, 29(3), 499-512; DOI:10.1080/00945719909349465

48. Verma K K, Dahiya R and Soni D, Synth React Inorg Met Org Chem., 1999, 29(6), 1033-1052; DOI:10.1080/00945719909349509

49. Verma K K and Dahiya R, Synth React Inorg Met Org Chem., 1999, 29(7), 12991314; DOI:10.1080/00945719909349529

50. Verma K K and Reena, Phosphorus, Sulfur Silicon Related Elements, 1999, 148(1), 227-234; DOI:10.1080/10426509908037013

51. Verma K K and Seema, Int J Chem Sci., 2008, 6, 371-380.

52. Srivastava S, Soni D K and Gupta H S, J Indian Chem Soc., 1996, 73, 255.

53. Narwal J K, Chhabra S, Malik R K, Garg S and Verma K K, Oriental J Chem., 2013, 29, 1339-1349.

54. Chhabra S and Verma K K, J Chem Pharm Res., 2010, 2(4), 569-575.

55. Vogel A I, A Test Book of Organic Chemistry, $3^{\text {rd }}$ Edn., Longman, London, 1975.

56. Weissberger A, Ed., Technique of Organic Chemistry, Vol. 7, $2^{\text {nd }}$ Edn., Interscience Publishers, Inc. N. Y., 1967.

57. Morgan G T and Kellet R E, J Chem Soc., 1926, 1080-1088; DOI:10.1039/JR9262901080

58. Petragnani $\mathrm{N}$ and Stefani $\mathrm{H}$ A, Tellurium in Organic Chemistry, $2^{\text {nd }}$ Edn., Academic Press, London, 2007, 67, 76.

59. Bergman J, Tetrahedron, 1972, 28(12), 3323-3331; DOI:10.1016/S0040-4020(01)93674-9

60. Khandelwal B L, Kumar K and Berry F J, Inorg Chim Acta, 1981, 99(2), 135-137; DOI:10.1016/S0020-1693(00)87958-X

61. Berry F J, Kustan E H, Roshani M and Smith B C, J Organometal Chem., 1975, 99(1), 115-117; DOI:10.1016/S0022-328X(00)86367-6

62. Khandelwal B L, Kumar K and Raina K, Synth React Inorg Met Org Chem., 1981, 11, 65-78.

63. Geary W J, Coord Chem Rev., 1971, 7(1), 81-122; DOI:10.1016/S0010-8545(00)80009-0 
64. Greenwood N N, Straughan B P and Wilson A E, J Chem Soc A, 1968, 2209-2212; DOI: $10.1039 / \mathrm{J} 19680002209$

65. Srivastava K P, Singh A and Singh S K, IOSR J Appl Chem., 2014, 7(4), 16-23; DOI:10.9790/5736-07411623

66. Baker A W and Shulgin A T, J Am Chem Soc., 1959, 81(7), 1523-1529; DOI:10.1021/ja01516a001

67. Freedman H H, J Am Chem Soc., 1961, 83(13), 2900-2905; DOI:10.1021/ja01474a026

68. Flett M St C, Spectrochim Acta, 1957, 10(1), 21-37; DOI:10.1016/0371-1951(57)80160-X

69. Bellamy I J, Infrared Spectra of Complex Molecules, Chapman and Hall Ltd. London, 1975.

70. Matijević-Sosa J, Vinković M and Vikić-Topić D, Croatica Chemica Acta., 2006, 79(3), 489-495.

71. Mishra A P and Soni M, Metal Baesd Drugs, 2008, 71, 243; DOI:10.1155/2008/875410

72. Aminabhavi T M, Biradar N S and Patil C S, Inorganica Chimica Acta., 1983, 78, 107-111; DOI:10.1016/S0020-1693(00)86498-1

73. Biradar N S, Aminabhavi T M and Patil C S, Inorganica Chimica Acta., 1983, 78, 47-50; DOI:10.1016/S0020-1693(00)86487-7

74. Tumer M, Celik C, Koksal H and Serin S, Transition Metal Chem., 1999, 24(5), 525532; DOI:10.1023/A:1006982622965

75. Gwaram N S, Mohd Ali H, Khaledi H, Abdulla M A, Hamid A, Hadi A, Kawi Lin T, Ching C L and Ooi C L, Molecules, 2012, 17(5), 5952;

DOI:10.3390/molecules17055952

76. Rudzinski W E and Aminabhavi T M, Inorganica Chimica Acta., 1982, 67, 177-182; DOI:10.1016/S0020-1693(00)85061-6

77. Osowole A A, Wakil S M and Alao O K, World Appl Sci J., 2015, 33(2), 336-342; DOI:10.5829/idosi.wasj.2015.33.02.22206

78. UR-Rehman S, Faiz A and Nawaz R, J Chil Chem Soc., 2013, 58(1), 1624-1627; DOI:10.4067/S0717-97072013000100026.

79. Mohamed G G and Abd El-Wahab Z H, J Thermal Anal Calorimetry, 2003, 73(1), 347-359; DOI:10.1023/A:1025126801265

80. Verma K K, Soni D and verma, Phosphorus, Sulfur Silicon, 2000, 166(1), 231-241; DOI:10.1080/10426500008076544

81. Pant B C, McWhinnie W R and Dance N S, J Organmetal Chem., 1973, 63, 305-310; DOI:10.1016/S0022-328X(73)80043-9

82. Srivastava T N, Singh J D, Indian J. Chem., 1987, 26A, 260.

83. Chauhan S, Garg S and Verma K K, Chem Sci Trans., 2016, 5(2), 431-441; DOI:10.7598/cst2016.1193

84. Kulkarani Y D, Srivastava S, Abdi S H R and Athar M, Synth React Inorg Met Org Chem., 1985, 15(8), 1043-1059; DOI:10.1080/00945718508060634

85. Chauhan S, Garg S and Verma K K, Res J Pharm Biol Chem Sci., 2016, 7(2), 265-274.

86. Aminabhavi T M and Rudzinski W E, Inorganica Chimica Acta., 1983, 76, L131L134; DOI:10.1016/S0020-1693(00)81478-4

87. Aminabhavi T M and Rudzinski W E, Inorganica Chimica Acta., 1983, 78, 51-55; DOI:10.1016/S0020-1693(00)86488-9

88. Cappuccino J C, Sherman N, Microbiology- A Laboratory Manual, Addison Wesley, California, 1999, 263.

89. Pharmacopoeia of India, Volume 1, Controller of Publications, Ministry of Health Department, Government of India, New Delhi, 2007, 37. 


\title{
Synthesis, Spectral and Biological Studies of Some Salicylidene-Trimethoprim Schiff Base Complexes of Aryltellurium(IV)
}

\author{
DEEPAK, K. K. VERMA and SAPANA GARG* \\ Department of Chemistry, Maharshi Dayanand University, Rohtak-124001, India \\ sapanagarg1511@gmail.com
}

Received 14 February 2017 / Accepted 2 March 2017

\begin{abstract}
A novel monobasic tridentate Schiff base salicylidene-trimethoprim, Sal-TMP, synthesized from trimethoprim and salicylaldehyde, form stable complexes with aryltellurium(IV) trichlorides and diaryltellurium(IV) dichlorides of the type Sal-TMP.ArTeCl ${ }_{2}$ and $\mathrm{Sal}_{-} \mathrm{TMP}_{\mathrm{Ar}} \mathrm{AeCl}$ (where $\mathrm{Ar}=p$-methoxyphenyl, $p$-ethoxyphenyl, $p$-hydroxyphenyl and 3-methyl-4-hydroxyphenyl). These have been characterized by elemental analyses, molar conductance, IR and ${ }^{1} \mathrm{H}$ NMR spectroscopy. The spectral studies predict the bonding of ligand through phenolic oxygen of Schiff base after deprotonation, nitrogen of the azomethine group and pyrimidine nitrogen to give hexacoordinated tellurium(IV) complexes. The complexes have also been screened for their antimicrobial activities against various bacteria and fungi organisms.
\end{abstract}

Keywords: Salicylidene-trimethoprim Schiff base, Aryltellurium(IV), Diaryltellurium(IV), Antibacterial, Antifungal activities

\section{Introduction}

Trimethoprim and its derivatives are broad spectrum antimicrobial agents with anti-parasitic activity $^{1-6}$ and were first described by Roth and coworker ${ }^{5}$. Schiff bases, also known as azomethine due to presence of $-\mathrm{C}=\mathrm{N}$ - group, play important role in biological system, such as anticancer $^{7-12}$, antibacterial ${ }^{13-15}$, antiviral ${ }^{16-18}$, antifungal ${ }^{19-21}$ and other biological properties $^{22-27}$ and also have been extensively used as ligands in coordination chemistry because of their excellent donor abilities as chelating agent ${ }^{28-32}$. Medicinal chemists have reported new derivatives of trimethoprim(TMZ) ${ }^{33-35}$ including the Schiff base derived from salicylaldehyde $2-4,36,37$.

Also, aryltellurium(IV) chlorides are known ${ }^{38-54}$ to act as Lewis acids and form complexes with several N-, O- and S- donor bases. In view of this, we herein report some new complexes derived from aryltellurium(IV) trichlorides, $\mathrm{RTeCl}_{3}$ and diaryltellurium(IV) dichlorides, $\mathrm{R}_{2} \mathrm{TeCl}_{2}$ with salicylidene-trimethoprim Schiff base(Sal-TMP). 


\section{Experimental}

All preparations were carried out under dry $\mathrm{N}_{2}$ atmosphere and the solvents used were purified by standard method ${ }^{55,56}$ before use. The purity of compounds was checked by thin layer chromatography using silica gel-G (Merck). Melting points were determined in open capillary tube and are uncorrected.

Carbon, hydrogen and nitrogen analyses were obtained microanalytically from SAIF, Panjab University Chandigarh on a ThermoFinnigan CHNS analyser. Conductivity was measured in DMSO at $25 \pm 2{ }^{\circ} \mathrm{C}$ with dip type conductivity cell on a microprocessor based conductivity bridge type MICROSIL.

IR $\left(4000-400 \mathrm{~cm}^{-1}\right)$ and far IR $\left(400-50 \mathrm{~cm}^{-1}\right)$ spectra were recorded in $\mathrm{KBr} /$ polyethylene pellets on a FT-Infrared spectrophotometer model RZX (Perkin Elmer) at SAIF, Panjab University Chandigarh. Proton magnetic resonance spectra were recorded in DMSO- $\mathrm{d}_{6}$ using TMS as an internal reference on BRUKER AVANCE II 400 NMR spectrometer. The antimicrobial screening was carried out by tube dilution method at Department of Pharmaceutical Sciences, M. D. University, Rohtak, India.

\section{Preparation of aryltellurium(IV) trichlorides and diaryltellurium(IV) dichlorides}

p-Methoxyphenyltellurium(IV) $\quad$ trichloride $^{57,58}, \quad$ bis(p-methoxyphenyl)tellurium(IV) dichloride ${ }^{58,59}, \quad p$-ethoxyphenyltellurium (IV) trichloride ${ }^{60}$, bis( $p$-ethoxyphenyl)tellurium dichloride $^{60} p$-hydroxyphenyltellurium(IV) trichloride ${ }^{61}$, bis( $p$-hydroxyphenyl) tellurium(IV) dichloride $^{61}$, 3-methyl-4-hydroxyphenyltellurium(IV) trichloride $^{62}$ and bis(3-methyl-4hydroxyphenyl)tellurium(IV) dichloride ${ }^{62}$ were prepared by the reactions of $\mathrm{TeCl}_{4}$ with anisole, phenetole, phenol, $o$-cresol respectively, by the methods reported in the literature ${ }^{57-62}$.

\section{Preparation of salicylidene-trimethoprim Schiff base (Sal-TMP)}

Equimolar quantity of saturated methanolic solution of drug and salicylaldehyde were mixed thoroughly. To this mixture $0.1 \%$ methanolic $\mathrm{KOH}$ was added to adjust the $\mathrm{pH}$ of the solution between 7-8 and was refluxed for 2 hours. A clear yellowish-green coloured solution was obtained. After completion of the reaction, the Schiff base ligand was isolated by crystallization after volume reduction by evaporation. The crystalline product was filtered and dried under vacuum and kept in desiccator over $\mathrm{P}_{4} \mathrm{O}_{10}$ until further use. Yield $=$ $80 \%$, M.pt.(decomp.) $=188-190{ }^{\circ} \mathrm{C}$. Analysis (Calculated) $\mathrm{C}_{21} \mathrm{H}_{22} \mathrm{~N}_{4} \mathrm{O}_{4}: \mathrm{C}(63.95), \mathrm{H}(5.62)$ and $\mathrm{N}(14.20)$; Found: $\mathrm{C}(63.50), \mathrm{H}(5.27)$ and $\mathrm{N}(13.99)$.

\section{Preparation of salicylidene-trimethoprim complexes of aryltellurium(IV) trichlorides} and diaryltellurium(IV) dichlorides

Aryltellurium(IV) trichlorides, $\mathrm{ArTeCl}_{3}$ and diaryltellurium(IV) dichlorides $\mathrm{Ar}_{2} \mathrm{TeCl}_{2}$ (Ar= $p$-methoxyphenyl, $p$-ethoxyphenyl, $p$-hydroxyphenyl and 3-methyl-4-hydroxyphenyl), when reacted with sodium salt of salicylidene-trimethoprim in equimolar ratio, yield SalTMP.ArTeCl $\mathrm{Cl}_{2}$ and Sal-TMP.Ar $\mathrm{TeCl}_{2} \mathrm{Type}$ complexes.

Sodium salt of the ligand was prepared by reacting equimolar (1:1) quantity of sodium metal and Schiff base in methanol. The solvent was distilled off to obtain sodium salt of Schiff base. Then a methanolic saturated solution of $2 \mathrm{mmol}$ of aryltellurium(IV) trichloride or diaryltellurium(IV) dichloride was added dropwise to suspension of $2 \mathrm{mmol}$ of sodium salt of Schiff base in about $50 \mathrm{~mL}$ benzene under reflux. The reaction mixture was further refluxed for 3-4 hours, cooled and precipitated sodium chloride was filtered off. The filtrate was then concentrated to about one third of original volume under reduced pressure and cooled in an ice bath to obtain coloured product. This was filtered, washed with benzene + methanol (1:1) and dried in vacuum desiccator over $\mathrm{P}_{4} \mathrm{O}_{10}$. 


\section{Results and Discussion}

$\mathrm{TeCl}_{4}$ when heated with anisole ${ }^{57-59}$, phenetole ${ }^{60}$, phenol ${ }^{61}, o$-cresol ${ }^{62}$ (Ar-H) appears to undergo Friedel-Crafts type condensation reaction whereby $\mathrm{TeCl}_{3}{ }^{+}$unit attacks a position para to the methoxy/ethoxy/hydroxy groups in the aromatic rings, thus resulting in the formation of aryltellurium(IV) trichlorides and diaryltellurium(IV) dichlorides.

$$
\begin{gathered}
\mathrm{Ar}-\mathrm{H}+\mathrm{TeCl}_{4} \longrightarrow \mathrm{ArTeCl}_{3}+\mathrm{HCl} \\
2 \mathrm{Ar}-\mathrm{H}+\mathrm{TeCl}_{4} \longrightarrow \mathrm{Ar}_{2} \mathrm{TeCl}_{2}+2 \mathrm{HCl}
\end{gathered}
$$

Preparation of salicylidene-trimethoprim Schiff base (Sal-TMP) by the reaction of trimethoprim drug and salicylaldehyde can be represented by following equations.

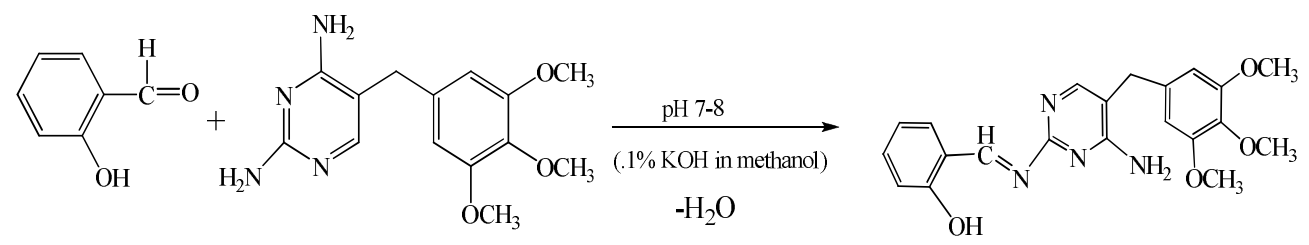

Sodium salt of salicylidene-trimethoprim Schiff base (Sal-TMP) reacts with aryltellurium(IV) trichlorides and diaryltellurium(IV) dichlorides in 1:1 molar ratio to yield the corresponding aryltellurium(IV) complexes.

$$
\begin{aligned}
& \mathrm{Sal}-\mathrm{TMP}+\mathrm{ArTeCl}_{3} \underset{\mathrm{NaCl}}{\stackrel{\mathrm{Na} / \mathrm{CH}_{3} \mathrm{OH}}{\longrightarrow}} \text { (Sal-TMP).ArTeCl } 2 \\
& \mathrm{Sal}-\mathrm{TMP}+\mathrm{Ar}_{2} \mathrm{TeCl}_{2} \underset{-\mathrm{NaCl}}{\stackrel{\mathrm{Na} / \mathrm{CH}_{3} \mathrm{OH}}{\longrightarrow}} \text { (Sal-TMP). } \mathrm{Ar}_{2} \mathrm{TeCl}_{2}
\end{aligned}
$$

All the tellurium(IV) complexes are coloured, crystalline solids, stable at room temperature and non-hygroscopic in nature. The complexes have been analysed for their tellurium, chlorine, carbon, hydrogen and nitrogen contents and the data along with their physical properties and yields are presented in Table 1.

\section{Conductance studies}

Molar conductance $\left(\Lambda_{M}\right)$ data for the complexes in DMSO are complied in Table 1. The $\Lambda_{M}$ value at $c a .10^{-3} \mathrm{M}$ for aryltellurium(IV) complexes in DMSO lie in the range 27.36-91.83 S $\mathrm{cm}^{2} \mathrm{~mol}^{-1}$ which predict the non electrolyte to $1: 1$ electrolyte $^{63,64}$ type behaviour of these complexes in DMSO, probably due to ionization into $\mathrm{ArTeCl}$.Sal- $\mathrm{TMP}^{+} / \mathrm{Ar}_{2} \mathrm{Te}$.Sal-TMP ${ }^{+}$ and $\mathrm{Cl}^{-}$in DMSO. This conductance behavoiur of tellurium(IV) salicylidene-trimethoprim Schiff base complexes is different from those of transition metal complexes ${ }^{65}$, which are reported to be non-electrolytes. The higher $\Lambda_{\mathrm{M}}$ values for some complexes may be due to steric factors and donor behaviour of DMSO to result in probable dissociation into solvated cation and $\mathrm{L}^{-}$along with $\mathrm{Cl}^{-}$in DMSO.

\section{Infrared spectra}

The IR data of Schiff base and its tellurium(IV) complexes are listed in Table 2. The spectra of Sal-TMP Schiff base complexes are quite complex and an attempt has therefore been made to identify the donor sites by comparing the spectra of complexes with parent ligand and $\mathrm{ArTeCl}_{3} / \mathrm{Ar}_{2} \mathrm{TeCl}_{2}$. 
Table 1. Analytical data, molar conductance and physical properties of salicylidene-trimethoprim Schiff base (Sal-TMP) complexes of tellurium(IV)

\begin{tabular}{|c|c|c|c|c|c|c|c|c|c|c|}
\hline \multirow[b]{2}{*}{$\begin{array}{l}\text { Compound } \\
\text { No. }\end{array}$} & \multirow[b]{2}{*}{$\begin{array}{l}\text { Complex } \\
\text { (Ar) }\end{array}$} & \multirow{2}{*}{$\begin{array}{c}\text { Empirical } \\
\text { formula } \\
\text { (Formula Wt.) }\end{array}$} & \multirow[b]{2}{*}{$\begin{array}{l}\text { Colour } \\
\text { (Yield, \%) }\end{array}$} & \multirow{2}{*}{$\begin{array}{l}\text { M. Pt. } \\
{ }^{\circ} \mathrm{C} \\
\text { dec. }\end{array}$} & \multicolumn{5}{|c|}{ Analyses \% Found (Calculated) } & \multirow{2}{*}{$\begin{array}{l}\Lambda_{\mathrm{M}} \text { at } c a \\
10^{-3} \mathrm{M} \mathrm{S} \\
\mathrm{cm}^{2} \mathrm{~mol}^{-1} \\
\text { in DMSO }\end{array}$} \\
\hline & & & & & $\mathrm{C}$ & $\mathrm{H}$ & $\mathrm{N}$ & $\mathrm{Te}$ & $\mathrm{Cl}$ & \\
\hline $\begin{array}{l}\text { Schiff } \\
\text { Base }\end{array}$ & Sal-TMP & $\mathrm{C}_{21} \mathrm{H}_{22} \mathrm{~N}_{4} \mathrm{O}_{4}$ & $\begin{array}{l}\text { Yellowish } \\
\text {-green }(80)\end{array}$ & $188-190$ & $\begin{array}{c}63.50 \\
(63.95)\end{array}$ & $\begin{array}{c}5.27 \\
(5.62)\end{array}$ & $\begin{array}{l}13.99 \\
(14.20)\end{array}$ & - & - & - \\
\hline I & $\begin{array}{l}\text { (Sal-TMP).ArTeCl }{ }_{2} \\
\text { (p-methoxyphenyl) }\end{array}$ & $\begin{array}{c}\mathrm{C}_{28} \mathrm{H}_{28} \mathrm{Cl}_{2} \mathrm{~N}_{4} \mathrm{O}_{5} \mathrm{Te} \\
(699.05)\end{array}$ & $\begin{array}{l}\text { Light cream } \\
(92)\end{array}$ & $230-232$ & $\begin{array}{c}47.88 \\
(48.11)\end{array}$ & $\begin{array}{c}3.99 \\
(4.04)\end{array}$ & $\begin{array}{c}7.75 \\
(8.01)\end{array}$ & $\begin{array}{c}18.07 \\
(18.24)\end{array}$ & $\begin{array}{c}9.89 \\
(10.01)\end{array}$ & 53.19 \\
\hline II & $\begin{array}{c}\text { (Sal-TMP).ArTeCl } \\
\text { (p-ethoxyphenyl) }\end{array}$ & $\begin{array}{c}\mathrm{C}_{29} \mathrm{H}_{30} \mathrm{Cl}_{2} \mathrm{~N}_{4} \mathrm{O}_{5} \mathrm{Te} \\
(713.08)\end{array}$ & $\begin{array}{c}\text { Cream } \\
(85)\end{array}$ & $258-260$ & $\begin{array}{c}48.12 \\
(48.85)\end{array}$ & $\begin{array}{c}3.87 \\
(4.24)\end{array}$ & $\begin{array}{l}7.52 \\
(7.86)\end{array}$ & $\begin{array}{c}17.38 \\
(17.89)\end{array}$ & $\begin{array}{c}9.52 \\
(9.81)\end{array}$ & 52.88 \\
\hline III & $\begin{array}{c}(\mathrm{Sal}-\mathrm{TMP}) \cdot \mathrm{ArTeCl}_{2} \\
(p \text {-hydroxyphenyl })\end{array}$ & $\begin{array}{c}\mathrm{C}_{27} \mathrm{H}_{26} \mathrm{Cl}_{2} \mathrm{~N}_{4} \mathrm{O}_{5} \mathrm{Te} \\
(685.03)\end{array}$ & $\begin{array}{l}\text { Dark cream } \\
\quad(94)\end{array}$ & $208-210$ & $\begin{array}{c}46.78 \\
(47.34)\end{array}$ & $\begin{array}{c}3.50 \\
(3.83)\end{array}$ & $\begin{array}{c}7.88 \\
(8.18)\end{array}$ & $\begin{array}{c}18.28 \\
(18.63)\end{array}$ & $\begin{array}{c}9.98 \\
(10.21)\end{array}$ & 55.73 \\
\hline IV & $\begin{array}{c}\text { (Sal-TMP).ArTeCl } \\
\text { (3-methyl-4- } \\
\text { hydroxyphenyl) }\end{array}$ & $\begin{array}{c}\mathrm{C}_{28} \mathrm{H}_{28} \mathrm{Cl}_{2} \mathrm{~N}_{4} \mathrm{O}_{5} \mathrm{Te} \\
(699.05)\end{array}$ & $\begin{array}{l}\text { Light } \\
\text { cream } \\
(80)\end{array}$ & $198-200$ & $\begin{array}{c}47.58 \\
(48.11)\end{array}$ & $\begin{array}{c}3.84 \\
(4.04)\end{array}$ & $\begin{array}{c}7.80 \\
(8.01)\end{array}$ & $\begin{array}{c}18.15 \\
(18.24)\end{array}$ & $\begin{array}{c}9.85 \\
(10.01)\end{array}$ & 58.68 \\
\hline $\mathrm{V}$ & $\begin{array}{c}(\mathrm{Sal}-\mathrm{TMP}) \cdot \mathrm{Ar}_{2} \mathrm{TeCl} \\
(p \text {-methoxyphenyl })\end{array}$ & $\begin{array}{c}\mathrm{C}_{35} \mathrm{H}_{35} \mathrm{ClN}_{4} \mathrm{O}_{6} \mathrm{Te} \\
(770.73)\end{array}$ & $\begin{array}{c}\text { Pale yellow } \\
\text { (84) }\end{array}$ & $130-132$ & $\begin{array}{c}53.84 \\
(54.54)\end{array}$ & $\begin{array}{c}4.31 \\
(4.58)\end{array}$ & $\begin{array}{c}7.11 \\
(7.27)\end{array}$ & $\begin{array}{c}16.43 \\
(16.56)\end{array}$ & $\begin{array}{c}4.42 \\
(4.60)\end{array}$ & 91.83 \\
\hline VI & $\begin{array}{c}\text { (Sal-TMP).Ar }{ }_{2} \mathrm{TeCl} \\
(p \text {-ethoxyphenyl) }\end{array}$ & $\begin{array}{c}\mathrm{C}_{37} \mathrm{H}_{39} \mathrm{ClN}_{4} \mathrm{O}_{6} \mathrm{Te} \\
(798.78)\end{array}$ & $\begin{array}{l}\text { Light yellow } \\
\text { (86) }\end{array}$ & $150-152$ & $\begin{array}{c}54.84 \\
(55.63)\end{array}$ & $\begin{array}{c}4.53 \\
(4.92)\end{array}$ & $\begin{array}{c}6.84 \\
(7.01)\end{array}$ & $\begin{array}{c}15.50 \\
(15.97)\end{array}$ & $\begin{array}{c}4.30 \\
(4.44)\end{array}$ & 35.90 \\
\hline VII & $\begin{array}{l}\text { (Sal-TMP).Ar } \mathrm{Ar}_{2} \mathrm{TeCl} \\
(p \text {-hydroxyphenyl) }\end{array}$ & $\begin{array}{c}\mathrm{C}_{33} \mathrm{H}_{31} \mathrm{ClN}_{4} \mathrm{O}_{6} \mathrm{Te} \\
(742.68)\end{array}$ & $\begin{array}{l}\text { Red } \\
(89)\end{array}$ & $146-148$ & $\begin{array}{c}52.84 \\
(53.37)\end{array}$ & $\begin{array}{c}4.01 \\
(4.21)\end{array}$ & $\begin{array}{c}7.27 \\
(7.54)\end{array}$ & $\begin{array}{c}16.89 \\
(17.18)\end{array}$ & $\begin{array}{c}4.52 \\
(4.77)\end{array}$ & 36.00 \\
\hline VIII & $\begin{array}{c}\text { (Sal-TMP). } \mathrm{Ar}_{2} \mathrm{TeCl} \\
\text { (3-methyl-4- } \\
\text { hydroxyphenyl) }\end{array}$ & $\begin{array}{c}\mathrm{C}_{35} \mathrm{H}_{35} \mathrm{ClN}_{4} \mathrm{O}_{6} \mathrm{Te} \\
(770.73)\end{array}$ & $\begin{array}{l}\text { Brown } \\
(78)\end{array}$ & $140-142$ & $\begin{array}{l}53.80 \\
(54.54)\end{array}$ & $\begin{array}{c}4.34 \\
(4.58)\end{array}$ & $\begin{array}{c}7.09 \\
(7.27)\end{array}$ & $\begin{array}{c}16.45 \\
(16.56)\end{array}$ & $\begin{array}{c}4.32 \\
(4.60)\end{array}$ & 27.36 \\
\hline
\end{tabular}


Table 2. Important infrared absorption bands $\left(\mathrm{cm}^{-1}\right)$ of Schiff base (Sal-TMP) and complexes

\begin{tabular}{|c|c|c|c|c|c|c|c|}
\hline Compound & $v_{(O-}$ & $v_{(1}$ & $v_{(C}$ & $v_{(C}$ & $v_{(\mathrm{C}-}$ & $v_{(\mathrm{Te}-\mathrm{N})}$ & $v_{(\mathrm{Te}}$ \\
\hline Sal-TMP & $2836 \mathrm{w}$ & $3317 \mathrm{~m} \mathrm{3119} \mathrm{m}$ & $1636 \mathrm{sh}$ & $1633 \mathrm{w} 1593 \mathrm{~s}$ & $1263 \mathrm{~s}$ & - & - \\
\hline $\bar{I}$ & - & $3323 \mathrm{~m} 3184 \mathrm{~m}$ & $1674 \mathrm{mb}$ & $1644 \mathrm{mb} 1587 \mathrm{~s}$ & $1341 \mathrm{~s}$ & $415 \mathrm{~m}$ & $288 \mathrm{w}$ \\
\hline II & - & $3323 \mathrm{~m} 3170 \mathrm{~m}$ & $1647 \mathrm{sh}$ & **1586 s & $1304 \mathrm{~s}$ & $420 \mathrm{~m}$ & $295 w$ \\
\hline III & 3405 m* & $3323 \mathrm{~m} 3150 \mathrm{~m}$ & $1674 \mathrm{mb}$ & $1641 \mathrm{mb} 1586 \mathrm{~s}$ & $1341 \mathrm{~s}$ & $419 \mathrm{~s}$ & $270 \mathrm{w}$ \\
\hline IV & 3398 m* & $3319 \mathrm{~m} 3172 \mathrm{~m}$ & $1652 \mathrm{mb}$ & 1649 & $1333 \mathrm{~s}$ & $450 \mathrm{~s}$ & $277 \mathrm{w}$ \\
\hline V & - & $3325 \mathrm{~m}$ & 164 & $* * 1$ & $1333 \mathrm{~s}$ & $416 \mathrm{~s}$ & $290 \mathrm{w}$ \\
\hline VI & - & $3320 \mathrm{~m} 3158 \mathrm{~m}$ & $1640 \mathrm{sh}$ & $* * 1590 \mathrm{~s}$ & $1331 \mathrm{~s}$ & $410 \mathrm{~m}$ & $273 w$ \\
\hline VII & $3401 \mathrm{w}^{*}$ & $3324 \mathrm{~m} 3168 \mathrm{~m}$ & $1674 \mathrm{mb}$ & $1643 \mathrm{mb} 1584 \mathrm{~s}$ & $1340 \mathrm{~s}$ & $418 \mathrm{~m}$ & $285 \mathrm{w}$ \\
\hline VIII & $3463 \mathrm{w}^{*}$ & $3304 \mathrm{~m} 3106 \mathrm{~m}$ & $1634 \mathrm{sh}$ & $1643 \mathrm{mb} 1584 \mathrm{~s}$ & $1340 \mathrm{~s}$ & $422 \mathrm{~m}$ & $287 \mathrm{w}$ \\
\hline
\end{tabular}

$s=$ sharp, m=medium, mb=medium broad, sh=shoulder, $w=w e a k$, "Due to phenolic $\mathrm{OH}$ of Rte and $\mathrm{R}_{2} \mathrm{Te}$ moieties; ${ }^{* *}$ band not resolved due to overlapping of band $\boldsymbol{v}_{(C=N)}$

Examination of the Schiff base (Sal-TMP) spectrum shows the presence of the hydrogen bonded conjugated- chelate ring system ${ }^{66-68}$ with its centre at $\sim 2832 \mathrm{~cm}^{-1}$. Thus intramolecular $\mathrm{H}$ - bonding is occurring by means of the formation of a quasi six- membered ring involving the $\mathrm{OH}----\mathrm{N}=\mathrm{C}$ bond.<smiles>C1=Cc2ccccc2O[NH+]=1</smiles>

Figure 1. Hydrogen bonding

Thus band disappear on chelation with aryltellurium(IV) chlorides ${ }^{69}$. Hydrogen bond contributes to planarity of the molecule which helps in chelation ${ }^{70}$. Also an intense ligand band at $1263 \mathrm{~cm}^{-1}$ (phenolic -C-O) in free ligand has shifted to higher frequency side in complexes. All these indicate that the hydroxyl group of salicylaldehyde of Schiff base is involved in coordination with tellurium ${ }^{71-73}$.

In addition, the spectra of the Schiff base shown shoulder at $1636 \mathrm{~cm}^{-1}$ with slightly resolved weak band at $1633 \mathrm{~cm}^{-1}$ and sharp band at $1593 \mathrm{~cm}^{-1}$ assigned to $v_{(\mathrm{C}=\mathrm{N})}$ mode for vibration of azomethine group ${ }^{2,4,65,74-76}$ and $v_{(\mathrm{C}=\mathrm{N}) \text { pyrim. For pyrimidine ring }}{ }^{4,77}$. These shift in aryltellurium Schiff base complexes towards higher and lower value ${ }^{74-79}$ reflecting that ligand coordinate through nitrogen atom of azomethine and pyrimidine ring.

The medium intensity band at $3317 \mathrm{~cm}^{-1}$ and $3119 \mathrm{~cm}^{-1}$ due to $v_{(\mathrm{N}-\mathrm{H})}$ asymmetric and symmetric vibrations respectively indicate the non involvement of the nitrogen atom of $\mathrm{NH}_{2}$ group attached to pyrimidine in coordination. The appearance of new weak bands around $270-295 \mathrm{~cm}^{-1}$ due to $v_{(\mathrm{Te}-\mathrm{O})}^{80-83}$ mode and medium to strong band in the range of 410-422 $\mathrm{cm}^{-1}$ due to $v_{(\mathrm{Te}-\mathrm{N})}$ mode ${ }^{84}$ further supports the involvement of phenolic oxygen (after deprotonation), azomethine and pyrimidine nitrogen atoms of Schiff base in the coordination.

Thus, IR data predict the tridentate nature of Sal-TMP involving azomethine nitrogen atom, phenolic oxygen after deprotonation and pyrimidine ring nitrogen giving rise to twosix and four membered chelate rings with the tellurium centre. 


\section{${ }^{1}$ H NMR spectra}

In order to identify the solution structure of Schiff base (Sal-TMP) and its complexes, ${ }^{1} \mathrm{H}$ NMR spectra were recorded in DMSO- $\mathrm{d}_{6}$ and given in Table 3. The proton resonance of the $\mathrm{OH}$ group at $10.92 \delta \mathrm{ppm}^{76,85}$ in Schiff base due to presence of intramolecular hydrogen bonding ${ }^{74}$ disappear on complexation indicating the involvement of phenolic oxygen in the coordination via deprotonation ${ }^{76}$. The azomethine protons which resonate as a singlet at $10.02 \delta \mathrm{ppm}$, the coordination of azomethine nitrogen to tellurium in the complexes are clearly demonstrated by a downfield shift of the peak ${ }^{86}$.

Table 3. ${ }^{1} \mathrm{H}$ NMR spectral data of Schiff base (Sal-TMP) and complexes in DMSO- $\mathrm{d}_{6}$

\begin{tabular}{|c|c|}
\hline Compound & Chemical Shift, $\delta$ ppm \\
\hline Sal-TMP & $\begin{array}{l}2.525(\mathrm{~s}, 2 \mathrm{H}, \text { methylene }), \quad 3.551-3.745\left(\mathrm{~s}, \quad 9 \mathrm{H},-\mathrm{OCH}_{3}\right), \quad 5.672(\mathrm{~s}, 1 \mathrm{H}, \\
\text { pyrimidine }), 6.508\left(\mathrm{~s}, 2 \mathrm{H},-\mathrm{NH}_{2}\right), 7.526-8.169(\mathrm{~m}, 6 \mathrm{H}, \text { aromatic proton), } \\
10.021(\mathrm{~s}, 1 \mathrm{H}, \text { azomethine }), 10.921(\mathrm{~s}, 1 \mathrm{H}, \mathrm{Schiff} \text { base } \mathrm{OH})\end{array}$ \\
\hline I & $\begin{array}{l}2.539(\mathrm{~s}, 2 \mathrm{H}, \text { methylene }), 3.598-3.840\left(\mathrm{~s}, 12 \mathrm{H},-\mathrm{OCH}_{3}\right), 6.574(\mathrm{~s}, 1 \mathrm{H}, \\
\text { pyrimidine }), 6.926\left(\mathrm{~s}, 2 \mathrm{H},-\mathrm{NH}_{2}\right), 7.383-7.522(\mathrm{~m}, 10 \mathrm{H}, \text { aromatic proton }), \\
10.209(\mathrm{~s}, 1 \mathrm{H}, \text { azomethine })\end{array}$ \\
\hline II & $\begin{array}{l}1.335\left(\mathrm{t}, 3 \mathrm{H},-\mathrm{OCH}_{2} \mathrm{CH}_{3}\right), 2.546(\mathrm{~s}, 2 \mathrm{H}, \text { methylene }), 3.963\left(\mathrm{q}, 2 \mathrm{H},-\mathrm{OCH}_{2} \mathrm{CH}_{3}\right) \text {, } \\
3.515-3.875\left(\mathrm{~s}, 9 \mathrm{H},-\mathrm{OCH}_{3}\right), 6.540(\mathrm{~s}, 1 \mathrm{H}, \text { pyrimidine }), 6.950\left(\mathrm{~s}, 2 \mathrm{H},-\mathrm{NH}_{2}\right), \\
6.993-7.809(\mathrm{~m}, 10 \mathrm{H} \text {, aromatic proton), } 10.193(\mathrm{~s}, 1 \mathrm{H} \text {, azomethine })\end{array}$ \\
\hline III & $\begin{array}{l}2.548(\mathrm{~s}, 2 \mathrm{H}, \quad \text { methylene }), \quad 3.601-3.783\left(\mathrm{~s}, 9 \mathrm{H},-\mathrm{OCH}_{3}\right), \quad 6.569(\mathrm{~s}, 1 \mathrm{H}, \\
\text { pyrimidine }), 6.914\left(\mathrm{~s}, 2 \mathrm{H},-\mathrm{NH}_{2}\right), 7.370-7.787(\mathrm{~m}, 10 \mathrm{H}, \text { aromatic proton }), \\
8.155(\mathrm{~s}, 1 \mathrm{H}, \text { phenolic } \mathrm{OH} \text { of } \mathrm{RTe}), 10.201(\mathrm{~s}, 1 \mathrm{H}, \text { azomethine })\end{array}$ \\
\hline IV & $\begin{array}{l}2.536(\mathrm{~s}, 2 \mathrm{H}, \text { methylene }), 2.527\left(\mathrm{~s}, 3 \mathrm{H},-\mathrm{CH}_{3}\right), 3.587-3.770\left(\mathrm{~s}, 9 \mathrm{H},-\mathrm{OCH}_{3}\right) \text {, } \\
\text { 6.605(s, } 1 \mathrm{H}, \text { pyrimidine }), 6.918\left(\mathrm{~s}, 2 \mathrm{H},-\mathrm{NH}_{2}\right), 7.051-7.671(\mathrm{~m}, 9 \mathrm{H} \text {, aromatic } \\
\text { proton }), 8.251(\mathrm{~s}, 1 \mathrm{H} \text {, phenolic } \mathrm{OH} \text { of } \mathrm{RTe}), 10.239(\mathrm{~s}, 1 \mathrm{H} \text {, azomethine })\end{array}$ \\
\hline V & $\begin{array}{l}2.528(\mathrm{~s}, 2 \mathrm{H}, \text { methylene }), 3.673-3.877\left(\mathrm{~s}, 15 \mathrm{H},-\mathrm{OCH}_{3}\right), 6.516(\mathrm{~s}, 1 \mathrm{H} \text {, } \\
\text { pyrimidine }), 6.882\left(\mathrm{~s}, 2 \mathrm{H},-\mathrm{NH}_{2}\right), 6.910-7.804(\mathrm{~m}, 14 \mathrm{H}, \text { aromatic proton }) \\
10.186(\mathrm{~s}, 1 \mathrm{H}, \text { azomethine })\end{array}$ \\
\hline VI & $\begin{array}{l}\left.\text { 1.363(t, } 6 \mathrm{H},-\mathrm{OCH}_{2} \mathbf{C H}_{3}\right), 2.531(\mathrm{~s}, 2 \mathrm{H}, \text { methylene }), 4.019\left(\mathrm{q}, 4 \mathrm{H},-\mathrm{OCH}_{2} \mathrm{CH}_{3}\right) \text {, } \\
3.555-3.765\left(\mathrm{~s}, 9 \mathrm{H},-\mathrm{OCH}_{3}\right), 6.510(\mathrm{~s}, 1 \mathrm{H}, \text { pyrimidine }), 6.924\left(\mathrm{~s}, 2 \mathrm{H},-\mathrm{NH}_{2}\right) \text {, } \\
\text { 7.524-7.805(m, } 14 \mathrm{H} \text {, aromatic proton }), 10.216(\mathrm{~s}, 1 \mathrm{H} \text {, azomethine })\end{array}$ \\
\hline VII & $\begin{array}{l}2.545(\mathrm{~s}, 2 \mathrm{H}, \quad \text { methylene }), 3.568-3.926\left(\mathrm{~s}, 9 \mathrm{H},-\mathrm{OCH}_{3}\right), 6.554(\mathrm{~s}, 1 \mathrm{H} \text {, } \\
\text { pyrimidine }), 6.884\left(\mathrm{~s}, 2 \mathrm{H},-\mathrm{NH}_{2}\right), 7.488-7.678(\mathrm{~m}, 14 \mathrm{H}, \text { aromatic proton), } \\
8.249\left(\mathrm{~s}, 2 \mathrm{H}, \text { phenolic } \mathrm{OH} \text { of } \mathrm{R}_{2} \mathrm{Te}\right), 10.238(\mathrm{~s}, 1 \mathrm{H}, \text { azomethine })\end{array}$ \\
\hline VIII & $\begin{array}{l}2.538(\mathrm{~s}, 2 \mathrm{H}, \text { methylene }), 2.529\left(\mathrm{~s}, 6 \mathrm{H},-\mathrm{CH}_{3}\right), 3.555-3.750\left(\mathrm{~s}, 9 \mathrm{H},-\mathrm{OCH}_{3}\right) \text {, } \\
6.502(\mathrm{~s}, 1 \mathrm{H}, \text { pyrimidine }), 6.926\left(\mathrm{~s}, 2 \mathrm{H},-\mathrm{NH}_{2}\right), 7.126-7.868(\mathrm{~m}, 12 \mathrm{H} \text {, aromatic } \\
\text { proton }), 8.149\left(\mathrm{~s}, 2 \mathrm{H} \text {, phenolic } \mathrm{OH} \text { of } \mathrm{R}_{2} \mathrm{Te}\right), 10.239(\mathrm{~s}, 1 \mathrm{H} \text {, azomethine })\end{array}$ \\
\hline
\end{tabular}
$s=$ singlet,$q=$ quartet,$t=$ triplet, $m=$ multiplet

The characterstic downfield shifting of proton signal in all complexes observed in region $5.67 \delta \mathrm{ppm}$ is due to pyrimidine proton in Schiff base clearly indicate the coordination through pyrimidine nitrogen atom ${ }^{87}$. The signal due to $-\mathrm{NH}$ proton is observed around $6.51 \delta \mathrm{ppm}$ which remain intact with slight variation in complexes is due to the proton bounded to nitrogen experience quadruple effect ${ }^{87}$. Thus salicylidene-trimethoprim act as a tridentate $-\mathrm{N},-\mathrm{N},-\mathrm{O}$ chelating ligand in Sal-TMP.ArTeCl $\mathrm{C}_{2}$ and Sal-TMP. $\mathrm{Ar}_{2} \mathrm{TeCl}$ complexes giving six coordinate tellurium having distorted octahedral geometry in these complexes as predicated from IR studies as well. The proposed structures are as given below (Figure 2). 


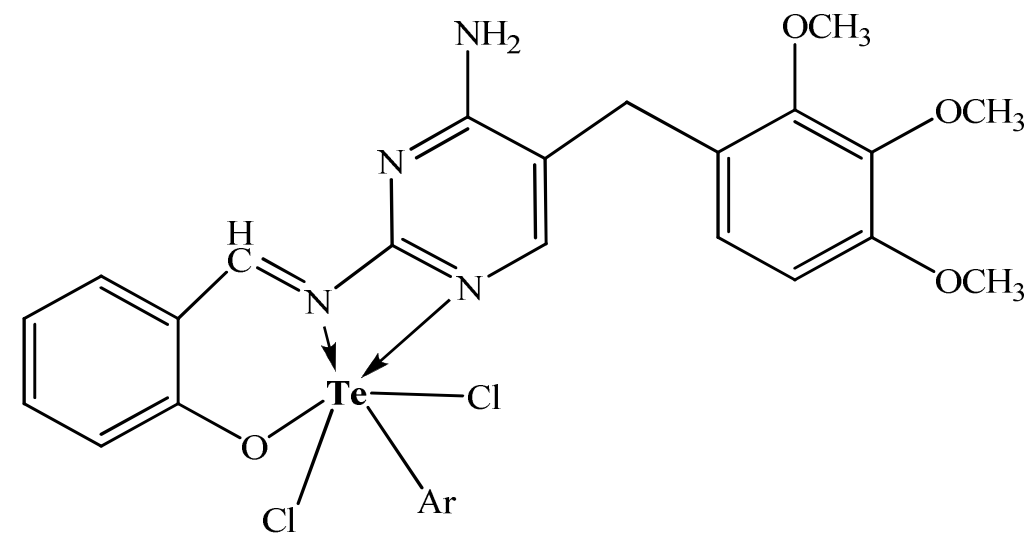<smiles></smiles>

Sal-TMP.Ar ${ }_{2} \mathrm{TeCl}$

$\mathrm{Ar}=p$-methoxyphenyl, $p$-ethoxyphenyl, $p$-hydroxyphenyl and 3-methyl-4-hydroxyphenyl

Figure 2. Proposed structures of complexes

\section{Biological Activity}

The salicylidene-trimethoprim Schiff base (Sal-TMP) and newly synthesized aryltellurium(IV) schiff base complexes were evaluated for their antimicrobial activity in vitro against Gram +ve bacteria (S. aureus ATCC 11632 and B. cereus MTCC 7350), Gram -ve bacteria (E. coli ATCC 35218, P. aeruginosa ATCC 23564, S. typhi ATCC 15499 and $P$. rettgeri DRDE) and fungal strains (A. niger, A. fumigates and A. flavus) by tube dilution method $^{88}$. Dilution of test and standard compounds were prepared double strength nutrient broth- I.P (Antibacterial) and Sabouraud Dextrose Broth -I.P (Antifungal) ${ }^{89}$. The samples were incubated at $37 \pm 1^{\circ} \mathrm{C}$ for $24 \mathrm{~h}$ (bacteria), $25 \pm 1^{\circ} \mathrm{C}$ for 7 days (A. niger), $30 \pm 1^{\circ} \mathrm{C}$ for 15 days (A. flavus), $35 \pm 1{ }^{\circ} \mathrm{C}$ for $72 \mathrm{~h}$ (A. fumigates) respectively and results were recorded in terms of MIC (The lowest concentration of test substances which inhibited values are presented in the Table 4.

The data show that the Sal-TMP complexes of aryltellurium(IV) exhibit more antimicrobial activity towards bacteria as compared to fungii. The antibacterial activity shows following trend. 
Sal-TMP.Ar $\mathrm{ArCl}_{2} \mathrm{Te}$ Sal-TMP.ArTeCl $\mathrm{Cl}_{2} \approx$ Sal-TMP Schiff base

Sal-TMP. $\mathrm{Ar}_{2} \mathrm{TeCl}$ and Sal-TMP.ArTeCl $\mathrm{Cl}_{2}$ complexes have activity towards $S$. typhi and more effectively against $B$. cereus but Schiff base does not show activity against these bacterial strains. Schiff base and its complexes show almost similar activity against fungal strains.

Table 4. Minimum Inhibitory Concentration, MIC, $\mu \mathrm{g} / \mathrm{mL}$; (-) Resistant

\begin{tabular}{|c|c|c|c|c|c|c|c|c|c|}
\hline \multirow[b]{2}{*}{ 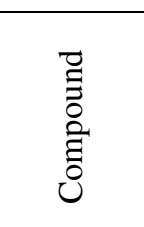 } & \multicolumn{6}{|c|}{ Bacteria strains } & \multicolumn{3}{|c|}{ Fungal strains } \\
\hline & $\begin{array}{c}S . \\
\text { aureus } \\
\text { (ATCC } \\
11632 \text { ) }\end{array}$ & $\begin{array}{l}\text { S. typhi } \\
\text { (ATCC } \\
15499 \text { ) }\end{array}$ & $\begin{array}{c}P . \\
\text { aeruginosa } \\
\text { (ATCC } \\
\text { 23564) }\end{array}$ & $\begin{array}{c}E . \\
a \quad c o l i \\
(\mathrm{ATCC} \\
35218)\end{array}$ & $\begin{array}{c}\text { B. cereus } \\
\text { (MTCC } \\
7350)\end{array}$ & $\begin{array}{c}P . \\
\text { rettgeri } \\
\text { (DRDE } \\
\text { strain) }\end{array}$ & A. niger & $\begin{array}{c}A . \\
\text { fumigates }\end{array}$ & $\begin{array}{l}\text { A. } \\
\text { flavus }\end{array}$ \\
\hline Sal-TMP & 2.5 & - & 1.25 & 5.0 & - & - & 20 & 5.0 & 1.25 \\
\hline I & 2.5 & - & 1.25 & - & 0.625 & 5.0 & - & - & - \\
\hline II & 5.0 & - & 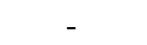 & - & 1.25 & 2.5 & 20 & 5.0 & 1.25 \\
\hline III & - & 20 & 10 & 5.0 & 1.25 & - & 5.0 & - & - \\
\hline IV & 1.25 & 2.5 & 1.25 & 5.0 & - & - & 5.0 & 10 & 5.0 \\
\hline V & - & 20 & 10 & 5.0 & 1.25 & - & 20 & - & - \\
\hline VI & 1.25 & 2.5 & 1.25 & 5.0 & - & - & - & 5.0 & - \\
\hline VII & 1.25 & - & 5.0 & 1.25 & 0.625 & 5.0 & - & 10 & - \\
\hline VIII & 2.5 & - & 1.25 & 5.0 & - & - & 20 & 5.0 & 1.25 \\
\hline
\end{tabular}

\section{Conclusion}

Aryltellurium(IV) and diaryltellurium(IV) dichlorides upon reaction with Schiff base(Sal-TMP) derived from salicylaldehyde and trimethoprim yield new complexes of tellurium(IV). The synthesized complexes were characterized by elemental analyses, conductance measurement, IR and ${ }^{1} \mathrm{H}$ NMR spectral studies. The analytical data suggest that the Sal-TMP Schiff base complexes have 1:1 stoichiometry. The Schiff bases (Sal-TMP) in these complexes functions as a uninegative tridentate ligand through azomethine nitrogen, phenolic oxygen after deprotonation and pyrimidine ring nitrogen atoms. Based on these studies, distorted octahedral geometry with two chelating rings has been assigned to these complexes. The complexes have been observed to possess substantial antimicrobial activity especially against bacteria.

\section{Acknowledgement}

The authors are grateful to M. D. University, Rohtak for providing the necessary facilities. One of the authors (Deepak) is also thankful to UGC New Delhi for providing fellowship. We also thank SAIF, Panjab University Chandigarh for providing the CHN analyses and spectral data.

\section{References}

1. Burchall J J, Corcoran J W and Hahn F E, Eds., Antibiotics, Springer, New York, NY, USA, $1975,3$.

2. Iqbal M S, Khan A H, Loothar B A and Bukhari I H, Med Chem Rev., 2009, 18, 31-42.

3. Blower P J, Аnnu Rep Prog Chem Sect A, 1999, 95, 631-655; DOI:10.1039/A808038K

4. Dhahir S A, Al-Sahib S A, Al Razaq W A and Kadhim N J, Baghdad Sci J., 2010, 7(1), 621.

5. Dr. Karl Thomae Gmb H, (Biberach an der Riss. DE), United States Patent. 4829058.

6. EL-Mossalamy E H, AL-Thabati S A, ALNomaiser F M, Commun Fac Sci Univ Ank Series B, 2005, 51(2), 21-30 
7. Sinha D, Tiwari A K, Singh S, Shukla G, Mishra P, Chandra H and Mishra A K, Eur J Med Chem., 2008, 43(1), 160-165; DOI:10.1016/j.ejmech.2007.03.022

8. Crowe A J, Smith P J and Atassi G, Chem Biol Interact., 1980, 32(1-2), 171-178; DOI:10.1016/0009-2797(80)90075-7

9. Wang M, Wang L F, Li Y Z, Li Q X, Xu Z D and Qu D M, Trans Met Chem., 2001, 26(3), 307-310; DOI:10.1023/A:1007159301849

10. Zishen Wu, Ziqi Gui and Zhenhuan Yen, Synth React Inorg Met Org Chem., 1990, 20(3), 335-344.

11. Desai S B, Desai P B and Desai K R, Hetrocycl Commun., 2001, 7(1), 83-90; DOI:10.1515/HC.2001.7.1.83

12. Dhumwad S D, Gudasi K B and Goudar T R, Indian J Chem., 1994, 33A, 320-324.

13. Przybylski P, Huczynski A, Pyta K, Brzezinski B and Bartl F, Curr Org Chem., 2009, 13(2), 124-148; DOI:10.2174/138527209787193774

14. Pandeya S N, Sriram D, Nath G and De Clercq E, Pharm Acta Hely., 1999, 74(1), 7-11.

15. Karthikeyan M S, Parsad D J, Poojary B, Bhat K S, Holla B S and Kumari N S, Bioorg. Med. Chem., 2006, 14(22), 7482-7489; DOI:10.1016/j.bmc.2006.07.015

16. Holla B S, Akberali P M and Shivananda M K, II Farmaco, 2001, 56(12), 919-927; DOI:10.1016/S0014-827X(01)01124-7

17. Jarrahpour A, Khalili D, De Clercq E, Salmi C and Brunel J M, Molecules, 2007, 12(8), 1720-1730; DOI:10.3390/12081720

18. Da Silva C M, da Silva D L, Modolo L V, Alves R B, de Resende, M A, Martins C V B and de Fatima A J, Adv Res., 2011, 2, 1-8; DOI:10.1016/j.jare.2010.05.004

19. Singh H, Yadav L D S and Mishra S B S, J Inorg Nucl Chem., 1981, 43(7), 17011704; DOI:10.1016/0022-1902(81)80367-3

20. Saravanan G, Pannerselvam P and Prakash C R, J Adv Pharm Techn Res., 2010, 1(3), 320-325; DOI:10.4103/0110-5558.72426

21. Panneerselvam P, Nair R R, Vijayalakshmi G, Subramanian E H and Sridhar S K, Eur. J. Med. Chem. 2005, 40, 225-229; DOI:10.1016/j.ejmech.2004.09.003

22. Singh N K and Singh S B, Indian J Chem., 2001, 40A, 1070-1075.

23. Walsh O M, Meegan M J, Prendergast R M, Nakib T A, Eur J Med Chem., 1996, 31, 989-1000; DOI:10.1016/S0223-5234(97)86178-8

24. Odabasoglu M, Arslan F, Ölmez H and Büyükgüngör O, Dyes and Pigments, 2007, 75(3), 507-515; DOI:10.1016/j.dyepig.2006.06.033

25. Vicini P, Geronikaki A, Incerti M, Busonera B, Poni G, Cabras C A and Colla P L, Bioorg Med Chem., 2003, 11(23), 4785-4789; DOI:10.1016/S09680896(03)00493-0

26. Pandeya S N, Sriram D, Nath G and DeClercq E, Eur J Pharm Sci., 1999, 9, 25-31.

27. Samadhiya S and Halve A, Orient J Chem., 2001, 17, 119-122.

28. Celik Ö, Ulusoy M, Tas E and Ide S, Anal Sciences, 2007, 23, x185.

29. Mahmoud M R, El-Gyar S A, Mousrafa A A and Shaker A, Polyhedron, 1987, 6(5), 1017-1020; DOI:10.1016/S0277-5387(00)80947-X

30. Qing-Yu H, Zheng-Hua M and Ya-Me Z, J Coord Chem., 1990, 21(3), 199-207; DOI:10.1080/00958979009409716

31. Cozzi P G, Chem Soc Rev., 2004, 33, 410-421; DOI:10.1039/B307853C

32. Tarafder M T H, Jin KT, Crouse K A, Ali A M. Yamin, B $M$ and Fun H K, Polyhedron, 2002, 21(25-26), 2547-2554; DOI:10.1016/S0277-5387(02)01188-9

33. Pandeya S N, Sriram D, Clercq E DE., Pannecouque C and Witvrouw M, Indian J Pharm Sci., 1998, 60(4), 207-212. 
34. Fleischman S H and Brooks C L, Proteins: Structure, Function and Genetics, 1990, 7, 52-61.

35. Suling W J, Reynolds R C, Barrow E W, Wilson L N, Piper J R and Barrow W W, J Antimicrobial Chemother., 1998, 42(6), 811-815; DOI:10.1093/jac/42.6.811

36. Gulcan M, Sonmez M and Berber I, Turkey J Chem., 2012, 36(1), 189-200.

37. Raman N, Kulandaisamy A and Jeyasubramanian K, Synth React Inorg Met Org Chem., 2001, 31(7), 1249-1270.

38. Wynne K J and Pearson P S, Inorg Chem., 1971, 10(12), 2735-2739; DOI:10.1021/ic50106a022

39. Wynne K J and Pearson P S, J Chem Soc Commun., 1970, 556-557; DOI:10.1039/C2970000556B

40. Wynne K J, Clark A J and Berg M, J Chem Soc Dalton Trans., 1972, 2370-2374; DOI:10.1039/DT9720002370

41. Clark E R, Collet A J and Naik D G, J Chem Soc Dalton Trans., 1973, 1961-1962; DOI:10.1039/DT9730001961

42. Berg M C, Diss Abstr Int., 1972, 33, 2982.

43. Srivastava T N, Singh M and Singh H B, Indian J Chem., 1982, 21A, 307-309.

44. Srivastava T N, Srivastava R C and Srivastava M, Indian J Chem., 1982, 21A, 539.

45. Srivastava T N, Srivastava R C and Srivastava V K, J Indian Chem Soc., 1983, 60, 891-892.

46. Garad M V, Polyhedron, 1985, 4(8), 1353-1355; DOI:10.1016/S0277-5387(00)86963-6

47. Verma K K and Reena, Synth React Inorg Met-Org Chem., 1999, 29(3), 499-512; DOI:10.1080/00945719909349465

48. Verma K K, Dahiya R and Soni D, Synth React Inorg Met Org Chem., 1999, 29(6), 1033-1052; DOI:10.1080/00945719909349509

49. Verma K K and Dahiya R, Synth React Inorg Met Org Chem., 1999, 29(7), 12991314; DOI:10.1080/00945719909349529

50. Verma K K and Reena, Phosphorus, Sulfur Silicon Related Elements, 1999, 148(1), 227-234; DOI:10.1080/10426509908037013

51. Verma K K and Seema, Int J Chem Sci., 2008, 6, 371-380.

52. Srivastava S, Soni D K and Gupta H S, J Indian Chem Soc., 1996, 73, 255.

53. Narwal J K, Chhabra S, Malik R K, Garg S and Verma K K, Oriental J Chem., 2013, 29, 1339-1349.

54. Chhabra S and Verma K K, J Chem Pharm Res., 2010, 2(4), 569-575.

55. Vogel A I, A Test Book of Organic Chemistry, $3^{\text {rd }}$ Edn., Longman, London, 1975.

56. Weissberger A, Ed., Technique of Organic Chemistry, Vol. 7, $2^{\text {nd }}$ Edn., Interscience Publishers, Inc. N. Y., 1967.

57. Morgan G T and Kellet R E, J Chem Soc., 1926, 1080-1088; DOI:10.1039/JR9262901080

58. Petragnani $\mathrm{N}$ and Stefani $\mathrm{H}$ A, Tellurium in Organic Chemistry, $2^{\text {nd }}$ Edn., Academic Press, London, 2007, 67, 76.

59. Bergman J, Tetrahedron, 1972, 28(12), 3323-3331; DOI:10.1016/S0040-4020(01)93674-9

60. Khandelwal B L, Kumar K and Berry F J, Inorg Chim Acta, 1981, 99(2), 135-137; DOI:10.1016/S0020-1693(00)87958-X

61. Berry F J, Kustan E H, Roshani M and Smith B C, J Organometal Chem., 1975, 99(1), 115-117; DOI:10.1016/S0022-328X(00)86367-6

62. Khandelwal B L, Kumar K and Raina K, Synth React Inorg Met Org Chem., 1981, 11, 65-78.

63. Geary W J, Coord Chem Rev., 1971, 7(1), 81-122; DOI:10.1016/S0010-8545(00)80009-0 
64. Greenwood N N, Straughan B P and Wilson A E, J Chem Soc A, 1968, 2209-2212; DOI: $10.1039 / \mathrm{J} 19680002209$

65. Srivastava K P, Singh A and Singh S K, IOSR J Appl Chem., 2014, 7(4), 16-23; DOI:10.9790/5736-07411623

66. Baker A W and Shulgin A T, J Am Chem Soc., 1959, 81(7), 1523-1529; DOI:10.1021/ja01516a001

67. Freedman H H, J Am Chem Soc., 1961, 83(13), 2900-2905; DOI:10.1021/ja01474a026

68. Flett M St C, Spectrochim Acta, 1957, 10(1), 21-37; DOI:10.1016/0371-1951(57)80160-X

69. Bellamy I J, Infrared Spectra of Complex Molecules, Chapman and Hall Ltd. London, 1975.

70. Matijević-Sosa J, Vinković M and Vikić-Topić D, Croatica Chemica Acta., 2006, 79(3), 489-495.

71. Mishra A P and Soni M, Metal Baesd Drugs, 2008, 71, 243; DOI:10.1155/2008/875410

72. Aminabhavi T M, Biradar N S and Patil C S, Inorganica Chimica Acta., 1983, 78, 107-111; DOI:10.1016/S0020-1693(00)86498-1

73. Biradar N S, Aminabhavi T M and Patil C S, Inorganica Chimica Acta., 1983, 78, 47-50; DOI:10.1016/S0020-1693(00)86487-7

74. Tumer M, Celik C, Koksal H and Serin S, Transition Metal Chem., 1999, 24(5), 525532; DOI:10.1023/A:1006982622965

75. Gwaram N S, Mohd Ali H, Khaledi H, Abdulla M A, Hamid A, Hadi A, Kawi Lin T, Ching C L and Ooi C L, Molecules, 2012, 17(5), 5952;

DOI:10.3390/molecules17055952

76. Rudzinski W E and Aminabhavi T M, Inorganica Chimica Acta., 1982, 67, 177-182; DOI:10.1016/S0020-1693(00)85061-6

77. Osowole A A, Wakil S M and Alao O K, World Appl Sci J., 2015, 33(2), 336-342; DOI:10.5829/idosi.wasj.2015.33.02.22206

78. UR-Rehman S, Faiz A and Nawaz R, J Chil Chem Soc., 2013, 58(1), 1624-1627; DOI:10.4067/S0717-97072013000100026.

79. Mohamed G G and Abd El-Wahab Z H, J Thermal Anal Calorimetry, 2003, 73(1), 347-359; DOI:10.1023/A:1025126801265

80. Verma K K, Soni D and verma, Phosphorus, Sulfur Silicon, 2000, 166(1), 231-241; DOI:10.1080/10426500008076544

81. Pant B C, McWhinnie W R and Dance N S, J Organmetal Chem., 1973, 63, 305-310; DOI:10.1016/S0022-328X(73)80043-9

82. Srivastava T N, Singh J D, Indian J. Chem., 1987, 26A, 260.

83. Chauhan S, Garg S and Verma K K, Chem Sci Trans., 2016, 5(2), 431-441; DOI:10.7598/cst2016.1193

84. Kulkarani Y D, Srivastava S, Abdi S H R and Athar M, Synth React Inorg Met Org Chem., 1985, 15(8), 1043-1059; DOI:10.1080/00945718508060634

85. Chauhan S, Garg S and Verma K K, Res J Pharm Biol Chem Sci., 2016, 7(2), 265-274.

86. Aminabhavi T M and Rudzinski W E, Inorganica Chimica Acta., 1983, 76, L131L134; DOI:10.1016/S0020-1693(00)81478-4

87. Aminabhavi T M and Rudzinski W E, Inorganica Chimica Acta., 1983, 78, 51-55; DOI:10.1016/S0020-1693(00)86488-9

88. Cappuccino J C, Sherman N, Microbiology- A Laboratory Manual, Addison Wesley, California, 1999, 263.

89. Pharmacopoeia of India, Volume 1, Controller of Publications, Ministry of Health Department, Government of India, New Delhi, 2007, 37. 


\title{
Synthesis, Spectral and Biological Studies of Some Salicylidene-Trimethoprim Schiff Base Complexes of Aryltellurium(IV)
}

\author{
DEEPAK, K. K. VERMA and SAPANA GARG* \\ Department of Chemistry, Maharshi Dayanand University, Rohtak-124001, India \\ sapanagarg1511@gmail.com
}

Received 14 February 2017 / Accepted 2 March 2017

\begin{abstract}
A novel monobasic tridentate Schiff base salicylidene-trimethoprim, Sal-TMP, synthesized from trimethoprim and salicylaldehyde, form stable complexes with aryltellurium(IV) trichlorides and diaryltellurium(IV) dichlorides of the type Sal-TMP.ArTeCl ${ }_{2}$ and $\mathrm{Sal}_{-} \mathrm{TMP}_{\mathrm{Ar}} \mathrm{AeCl}$ (where $\mathrm{Ar}=p$-methoxyphenyl, $p$-ethoxyphenyl, $p$-hydroxyphenyl and 3-methyl-4-hydroxyphenyl). These have been characterized by elemental analyses, molar conductance, IR and ${ }^{1} \mathrm{H}$ NMR spectroscopy. The spectral studies predict the bonding of ligand through phenolic oxygen of Schiff base after deprotonation, nitrogen of the azomethine group and pyrimidine nitrogen to give hexacoordinated tellurium(IV) complexes. The complexes have also been screened for their antimicrobial activities against various bacteria and fungi organisms.
\end{abstract}

Keywords: Salicylidene-trimethoprim Schiff base, Aryltellurium(IV), Diaryltellurium(IV), Antibacterial, Antifungal activities

\section{Introduction}

Trimethoprim and its derivatives are broad spectrum antimicrobial agents with anti-parasitic activity $^{1-6}$ and were first described by Roth and coworker ${ }^{5}$. Schiff bases, also known as azomethine due to presence of $-\mathrm{C}=\mathrm{N}$ - group, play important role in biological system, such as anticancer $^{7-12}$, antibacterial ${ }^{13-15}$, antiviral ${ }^{16-18}$, antifungal ${ }^{19-21}$ and other biological properties $^{22-27}$ and also have been extensively used as ligands in coordination chemistry because of their excellent donor abilities as chelating agent ${ }^{28-32}$. Medicinal chemists have reported new derivatives of trimethoprim(TMZ) ${ }^{33-35}$ including the Schiff base derived from salicylaldehyde $2-4,36,37$.

Also, aryltellurium(IV) chlorides are known ${ }^{38-54}$ to act as Lewis acids and form complexes with several N-, O- and S- donor bases. In view of this, we herein report some new complexes derived from aryltellurium(IV) trichlorides, $\mathrm{RTeCl}_{3}$ and diaryltellurium(IV) dichlorides, $\mathrm{R}_{2} \mathrm{TeCl}_{2}$ with salicylidene-trimethoprim Schiff base(Sal-TMP). 


\section{Experimental}

All preparations were carried out under dry $\mathrm{N}_{2}$ atmosphere and the solvents used were purified by standard method ${ }^{55,56}$ before use. The purity of compounds was checked by thin layer chromatography using silica gel-G (Merck). Melting points were determined in open capillary tube and are uncorrected.

Carbon, hydrogen and nitrogen analyses were obtained microanalytically from SAIF, Panjab University Chandigarh on a ThermoFinnigan CHNS analyser. Conductivity was measured in DMSO at $25 \pm 2{ }^{\circ} \mathrm{C}$ with dip type conductivity cell on a microprocessor based conductivity bridge type MICROSIL.

IR $\left(4000-400 \mathrm{~cm}^{-1}\right)$ and far IR $\left(400-50 \mathrm{~cm}^{-1}\right)$ spectra were recorded in $\mathrm{KBr} /$ polyethylene pellets on a FT-Infrared spectrophotometer model RZX (Perkin Elmer) at SAIF, Panjab University Chandigarh. Proton magnetic resonance spectra were recorded in DMSO- $\mathrm{d}_{6}$ using TMS as an internal reference on BRUKER AVANCE II 400 NMR spectrometer. The antimicrobial screening was carried out by tube dilution method at Department of Pharmaceutical Sciences, M. D. University, Rohtak, India.

\section{Preparation of aryltellurium(IV) trichlorides and diaryltellurium(IV) dichlorides}

p-Methoxyphenyltellurium(IV) $\quad$ trichloride $^{57,58}, \quad$ bis(p-methoxyphenyl)tellurium(IV) dichloride ${ }^{58,59}, \quad p$-ethoxyphenyltellurium (IV) trichloride ${ }^{60}$, bis( $p$-ethoxyphenyl)tellurium dichloride $^{60} p$-hydroxyphenyltellurium(IV) trichloride ${ }^{61}$, bis( $p$-hydroxyphenyl) tellurium(IV) dichloride $^{61}$, 3-methyl-4-hydroxyphenyltellurium(IV) trichloride $^{62}$ and bis(3-methyl-4hydroxyphenyl)tellurium(IV) dichloride ${ }^{62}$ were prepared by the reactions of $\mathrm{TeCl}_{4}$ with anisole, phenetole, phenol, $o$-cresol respectively, by the methods reported in the literature ${ }^{57-62}$.

\section{Preparation of salicylidene-trimethoprim Schiff base (Sal-TMP)}

Equimolar quantity of saturated methanolic solution of drug and salicylaldehyde were mixed thoroughly. To this mixture $0.1 \%$ methanolic $\mathrm{KOH}$ was added to adjust the $\mathrm{pH}$ of the solution between 7-8 and was refluxed for 2 hours. A clear yellowish-green coloured solution was obtained. After completion of the reaction, the Schiff base ligand was isolated by crystallization after volume reduction by evaporation. The crystalline product was filtered and dried under vacuum and kept in desiccator over $\mathrm{P}_{4} \mathrm{O}_{10}$ until further use. Yield $=$ $80 \%$, M.pt.(decomp.) $=188-190{ }^{\circ} \mathrm{C}$. Analysis (Calculated) $\mathrm{C}_{21} \mathrm{H}_{22} \mathrm{~N}_{4} \mathrm{O}_{4}: \mathrm{C}(63.95), \mathrm{H}(5.62)$ and $\mathrm{N}(14.20)$; Found: $\mathrm{C}(63.50), \mathrm{H}(5.27)$ and $\mathrm{N}(13.99)$.

\section{Preparation of salicylidene-trimethoprim complexes of aryltellurium(IV) trichlorides} and diaryltellurium(IV) dichlorides

Aryltellurium(IV) trichlorides, $\mathrm{ArTeCl}_{3}$ and diaryltellurium(IV) dichlorides $\mathrm{Ar}_{2} \mathrm{TeCl}_{2}$ (Ar= $p$-methoxyphenyl, $p$-ethoxyphenyl, $p$-hydroxyphenyl and 3-methyl-4-hydroxyphenyl), when reacted with sodium salt of salicylidene-trimethoprim in equimolar ratio, yield SalTMP.ArTeCl $\mathrm{Cl}_{2}$ and Sal-TMP.Ar $\mathrm{TeCl}_{2} \mathrm{Type}$ complexes.

Sodium salt of the ligand was prepared by reacting equimolar (1:1) quantity of sodium metal and Schiff base in methanol. The solvent was distilled off to obtain sodium salt of Schiff base. Then a methanolic saturated solution of $2 \mathrm{mmol}$ of aryltellurium(IV) trichloride or diaryltellurium(IV) dichloride was added dropwise to suspension of $2 \mathrm{mmol}$ of sodium salt of Schiff base in about $50 \mathrm{~mL}$ benzene under reflux. The reaction mixture was further refluxed for 3-4 hours, cooled and precipitated sodium chloride was filtered off. The filtrate was then concentrated to about one third of original volume under reduced pressure and cooled in an ice bath to obtain coloured product. This was filtered, washed with benzene + methanol (1:1) and dried in vacuum desiccator over $\mathrm{P}_{4} \mathrm{O}_{10}$. 


\section{Results and Discussion}

$\mathrm{TeCl}_{4}$ when heated with anisole ${ }^{57-59}$, phenetole ${ }^{60}$, phenol ${ }^{61}, o$-cresol ${ }^{62}$ (Ar-H) appears to undergo Friedel-Crafts type condensation reaction whereby $\mathrm{TeCl}_{3}{ }^{+}$unit attacks a position para to the methoxy/ethoxy/hydroxy groups in the aromatic rings, thus resulting in the formation of aryltellurium(IV) trichlorides and diaryltellurium(IV) dichlorides.

$$
\begin{gathered}
\mathrm{Ar}-\mathrm{H}+\mathrm{TeCl}_{4} \longrightarrow \mathrm{ArTeCl}_{3}+\mathrm{HCl} \\
2 \mathrm{Ar}-\mathrm{H}+\mathrm{TeCl}_{4} \longrightarrow \mathrm{Ar}_{2} \mathrm{TeCl}_{2}+2 \mathrm{HCl}
\end{gathered}
$$

Preparation of salicylidene-trimethoprim Schiff base (Sal-TMP) by the reaction of trimethoprim drug and salicylaldehyde can be represented by following equations.

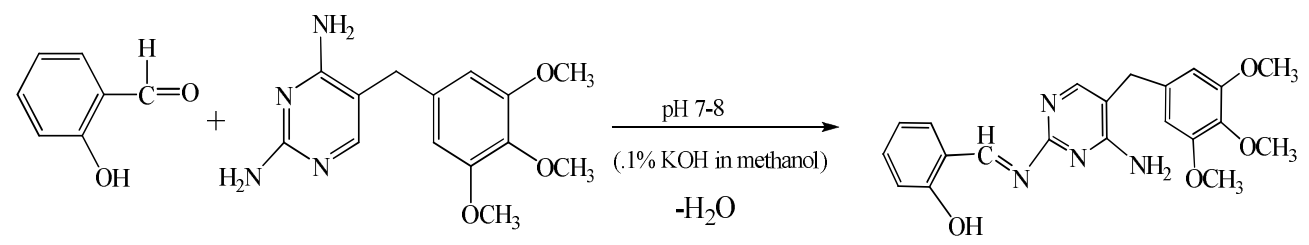

Sodium salt of salicylidene-trimethoprim Schiff base (Sal-TMP) reacts with aryltellurium(IV) trichlorides and diaryltellurium(IV) dichlorides in 1:1 molar ratio to yield the corresponding aryltellurium(IV) complexes.

$$
\begin{aligned}
& \mathrm{Sal}-\mathrm{TMP}+\mathrm{ArTeCl}_{3} \underset{\mathrm{NaCl}}{\stackrel{\mathrm{Na} / \mathrm{CH}_{3} \mathrm{OH}}{\longrightarrow}} \text { (Sal-TMP).ArTeCl } 2 \\
& \mathrm{Sal}-\mathrm{TMP}+\mathrm{Ar}_{2} \mathrm{TeCl}_{2} \underset{-\mathrm{NaCl}}{\stackrel{\mathrm{Na} / \mathrm{CH}_{3} \mathrm{OH}}{\longrightarrow}} \text { (Sal-TMP). } \mathrm{Ar}_{2} \mathrm{TeCl}_{2}
\end{aligned}
$$

All the tellurium(IV) complexes are coloured, crystalline solids, stable at room temperature and non-hygroscopic in nature. The complexes have been analysed for their tellurium, chlorine, carbon, hydrogen and nitrogen contents and the data along with their physical properties and yields are presented in Table 1.

\section{Conductance studies}

Molar conductance $\left(\Lambda_{M}\right)$ data for the complexes in DMSO are complied in Table 1. The $\Lambda_{M}$ value at $c a .10^{-3} \mathrm{M}$ for aryltellurium(IV) complexes in DMSO lie in the range 27.36-91.83 S $\mathrm{cm}^{2} \mathrm{~mol}^{-1}$ which predict the non electrolyte to $1: 1$ electrolyte $^{63,64}$ type behaviour of these complexes in DMSO, probably due to ionization into $\mathrm{ArTeCl}$.Sal- $\mathrm{TMP}^{+} / \mathrm{Ar}_{2} \mathrm{Te}$.Sal-TMP ${ }^{+}$ and $\mathrm{Cl}^{-}$in DMSO. This conductance behavoiur of tellurium(IV) salicylidene-trimethoprim Schiff base complexes is different from those of transition metal complexes ${ }^{65}$, which are reported to be non-electrolytes. The higher $\Lambda_{\mathrm{M}}$ values for some complexes may be due to steric factors and donor behaviour of DMSO to result in probable dissociation into solvated cation and $\mathrm{L}^{-}$along with $\mathrm{Cl}^{-}$in DMSO.

\section{Infrared spectra}

The IR data of Schiff base and its tellurium(IV) complexes are listed in Table 2. The spectra of Sal-TMP Schiff base complexes are quite complex and an attempt has therefore been made to identify the donor sites by comparing the spectra of complexes with parent ligand and $\mathrm{ArTeCl}_{3} / \mathrm{Ar}_{2} \mathrm{TeCl}_{2}$. 
Table 1. Analytical data, molar conductance and physical properties of salicylidene-trimethoprim Schiff base (Sal-TMP) complexes of tellurium(IV)

\begin{tabular}{|c|c|c|c|c|c|c|c|c|c|c|}
\hline \multirow[b]{2}{*}{$\begin{array}{l}\text { Compound } \\
\text { No. }\end{array}$} & \multirow[b]{2}{*}{$\begin{array}{l}\text { Complex } \\
\text { (Ar) }\end{array}$} & \multirow{2}{*}{$\begin{array}{c}\text { Empirical } \\
\text { formula } \\
\text { (Formula Wt.) }\end{array}$} & \multirow[b]{2}{*}{$\begin{array}{l}\text { Colour } \\
\text { (Yield, \%) }\end{array}$} & \multirow{2}{*}{$\begin{array}{l}\text { M. Pt. } \\
{ }^{\circ} \mathrm{C} \\
\text { dec. }\end{array}$} & \multicolumn{5}{|c|}{ Analyses \% Found (Calculated) } & \multirow{2}{*}{$\begin{array}{l}\Lambda_{\mathrm{M}} \text { at } c a \\
10^{-3} \mathrm{M} \mathrm{S} \\
\mathrm{cm}^{2} \mathrm{~mol}^{-1} \\
\text { in DMSO }\end{array}$} \\
\hline & & & & & $\mathrm{C}$ & $\mathrm{H}$ & $\mathrm{N}$ & $\mathrm{Te}$ & $\mathrm{Cl}$ & \\
\hline $\begin{array}{l}\text { Schiff } \\
\text { Base }\end{array}$ & Sal-TMP & $\mathrm{C}_{21} \mathrm{H}_{22} \mathrm{~N}_{4} \mathrm{O}_{4}$ & $\begin{array}{l}\text { Yellowish } \\
\text {-green }(80)\end{array}$ & $188-190$ & $\begin{array}{c}63.50 \\
(63.95)\end{array}$ & $\begin{array}{c}5.27 \\
(5.62)\end{array}$ & $\begin{array}{l}13.99 \\
(14.20)\end{array}$ & - & - & - \\
\hline I & $\begin{array}{l}\text { (Sal-TMP).ArTeCl }{ }_{2} \\
\text { (p-methoxyphenyl) }\end{array}$ & $\begin{array}{c}\mathrm{C}_{28} \mathrm{H}_{28} \mathrm{Cl}_{2} \mathrm{~N}_{4} \mathrm{O}_{5} \mathrm{Te} \\
(699.05)\end{array}$ & $\begin{array}{l}\text { Light cream } \\
(92)\end{array}$ & $230-232$ & $\begin{array}{c}47.88 \\
(48.11)\end{array}$ & $\begin{array}{c}3.99 \\
(4.04)\end{array}$ & $\begin{array}{c}7.75 \\
(8.01)\end{array}$ & $\begin{array}{c}18.07 \\
(18.24)\end{array}$ & $\begin{array}{c}9.89 \\
(10.01)\end{array}$ & 53.19 \\
\hline II & $\begin{array}{c}\text { (Sal-TMP).ArTeCl } \\
\text { (p-ethoxyphenyl) }\end{array}$ & $\begin{array}{c}\mathrm{C}_{29} \mathrm{H}_{30} \mathrm{Cl}_{2} \mathrm{~N}_{4} \mathrm{O}_{5} \mathrm{Te} \\
(713.08)\end{array}$ & $\begin{array}{c}\text { Cream } \\
(85)\end{array}$ & $258-260$ & $\begin{array}{c}48.12 \\
(48.85)\end{array}$ & $\begin{array}{c}3.87 \\
(4.24)\end{array}$ & $\begin{array}{l}7.52 \\
(7.86)\end{array}$ & $\begin{array}{c}17.38 \\
(17.89)\end{array}$ & $\begin{array}{c}9.52 \\
(9.81)\end{array}$ & 52.88 \\
\hline III & $\begin{array}{c}(\mathrm{Sal}-\mathrm{TMP}) \cdot \mathrm{ArTeCl}_{2} \\
(p \text {-hydroxyphenyl })\end{array}$ & $\begin{array}{c}\mathrm{C}_{27} \mathrm{H}_{26} \mathrm{Cl}_{2} \mathrm{~N}_{4} \mathrm{O}_{5} \mathrm{Te} \\
(685.03)\end{array}$ & $\begin{array}{l}\text { Dark cream } \\
\quad(94)\end{array}$ & $208-210$ & $\begin{array}{c}46.78 \\
(47.34)\end{array}$ & $\begin{array}{c}3.50 \\
(3.83)\end{array}$ & $\begin{array}{c}7.88 \\
(8.18)\end{array}$ & $\begin{array}{c}18.28 \\
(18.63)\end{array}$ & $\begin{array}{c}9.98 \\
(10.21)\end{array}$ & 55.73 \\
\hline IV & $\begin{array}{c}\text { (Sal-TMP).ArTeCl } \\
\text { (3-methyl-4- } \\
\text { hydroxyphenyl) }\end{array}$ & $\begin{array}{c}\mathrm{C}_{28} \mathrm{H}_{28} \mathrm{Cl}_{2} \mathrm{~N}_{4} \mathrm{O}_{5} \mathrm{Te} \\
(699.05)\end{array}$ & $\begin{array}{l}\text { Light } \\
\text { cream } \\
(80)\end{array}$ & $198-200$ & $\begin{array}{c}47.58 \\
(48.11)\end{array}$ & $\begin{array}{c}3.84 \\
(4.04)\end{array}$ & $\begin{array}{c}7.80 \\
(8.01)\end{array}$ & $\begin{array}{c}18.15 \\
(18.24)\end{array}$ & $\begin{array}{c}9.85 \\
(10.01)\end{array}$ & 58.68 \\
\hline $\mathrm{V}$ & $\begin{array}{c}(\mathrm{Sal}-\mathrm{TMP}) \cdot \mathrm{Ar}_{2} \mathrm{TeCl} \\
(p \text {-methoxyphenyl })\end{array}$ & $\begin{array}{c}\mathrm{C}_{35} \mathrm{H}_{35} \mathrm{ClN}_{4} \mathrm{O}_{6} \mathrm{Te} \\
(770.73)\end{array}$ & $\begin{array}{c}\text { Pale yellow } \\
\text { (84) }\end{array}$ & $130-132$ & $\begin{array}{c}53.84 \\
(54.54)\end{array}$ & $\begin{array}{c}4.31 \\
(4.58)\end{array}$ & $\begin{array}{c}7.11 \\
(7.27)\end{array}$ & $\begin{array}{c}16.43 \\
(16.56)\end{array}$ & $\begin{array}{c}4.42 \\
(4.60)\end{array}$ & 91.83 \\
\hline VI & $\begin{array}{c}\text { (Sal-TMP).Ar }{ }_{2} \mathrm{TeCl} \\
(p \text {-ethoxyphenyl) }\end{array}$ & $\begin{array}{c}\mathrm{C}_{37} \mathrm{H}_{39} \mathrm{ClN}_{4} \mathrm{O}_{6} \mathrm{Te} \\
(798.78)\end{array}$ & $\begin{array}{l}\text { Light yellow } \\
\text { (86) }\end{array}$ & $150-152$ & $\begin{array}{c}54.84 \\
(55.63)\end{array}$ & $\begin{array}{c}4.53 \\
(4.92)\end{array}$ & $\begin{array}{c}6.84 \\
(7.01)\end{array}$ & $\begin{array}{c}15.50 \\
(15.97)\end{array}$ & $\begin{array}{c}4.30 \\
(4.44)\end{array}$ & 35.90 \\
\hline VII & $\begin{array}{l}\text { (Sal-TMP).Ar } \mathrm{Ar}_{2} \mathrm{TeCl} \\
(p \text {-hydroxyphenyl) }\end{array}$ & $\begin{array}{c}\mathrm{C}_{33} \mathrm{H}_{31} \mathrm{ClN}_{4} \mathrm{O}_{6} \mathrm{Te} \\
(742.68)\end{array}$ & $\begin{array}{l}\text { Red } \\
(89)\end{array}$ & $146-148$ & $\begin{array}{c}52.84 \\
(53.37)\end{array}$ & $\begin{array}{c}4.01 \\
(4.21)\end{array}$ & $\begin{array}{c}7.27 \\
(7.54)\end{array}$ & $\begin{array}{c}16.89 \\
(17.18)\end{array}$ & $\begin{array}{c}4.52 \\
(4.77)\end{array}$ & 36.00 \\
\hline VIII & $\begin{array}{c}\text { (Sal-TMP). } \mathrm{Ar}_{2} \mathrm{TeCl} \\
\text { (3-methyl-4- } \\
\text { hydroxyphenyl) }\end{array}$ & $\begin{array}{c}\mathrm{C}_{35} \mathrm{H}_{35} \mathrm{ClN}_{4} \mathrm{O}_{6} \mathrm{Te} \\
(770.73)\end{array}$ & $\begin{array}{l}\text { Brown } \\
(78)\end{array}$ & $140-142$ & $\begin{array}{l}53.80 \\
(54.54)\end{array}$ & $\begin{array}{c}4.34 \\
(4.58)\end{array}$ & $\begin{array}{c}7.09 \\
(7.27)\end{array}$ & $\begin{array}{c}16.45 \\
(16.56)\end{array}$ & $\begin{array}{c}4.32 \\
(4.60)\end{array}$ & 27.36 \\
\hline
\end{tabular}


Table 2. Important infrared absorption bands $\left(\mathrm{cm}^{-1}\right)$ of Schiff base (Sal-TMP) and complexes

\begin{tabular}{|c|c|c|c|c|c|c|c|}
\hline Compound & $v_{(O-}$ & $v_{(1}$ & $v_{(C}$ & $v_{(C}$ & $v_{(\mathrm{C}-}$ & $v_{(\mathrm{Te}-\mathrm{N})}$ & $v_{(\mathrm{Te}}$ \\
\hline Sal-TMP & $2836 \mathrm{w}$ & $3317 \mathrm{~m} \mathrm{3119} \mathrm{m}$ & $1636 \mathrm{sh}$ & $1633 \mathrm{w} 1593 \mathrm{~s}$ & $1263 \mathrm{~s}$ & - & - \\
\hline $\bar{I}$ & - & $3323 \mathrm{~m} 3184 \mathrm{~m}$ & $1674 \mathrm{mb}$ & $1644 \mathrm{mb} 1587 \mathrm{~s}$ & $1341 \mathrm{~s}$ & $415 \mathrm{~m}$ & $288 \mathrm{w}$ \\
\hline II & - & $3323 \mathrm{~m} 3170 \mathrm{~m}$ & $1647 \mathrm{sh}$ & **1586 s & $1304 \mathrm{~s}$ & $420 \mathrm{~m}$ & $295 w$ \\
\hline III & 3405 m* & $3323 \mathrm{~m} 3150 \mathrm{~m}$ & $1674 \mathrm{mb}$ & $1641 \mathrm{mb} 1586 \mathrm{~s}$ & $1341 \mathrm{~s}$ & $419 \mathrm{~s}$ & $270 \mathrm{w}$ \\
\hline IV & 3398 m* & $3319 \mathrm{~m} 3172 \mathrm{~m}$ & $1652 \mathrm{mb}$ & 1649 & $1333 \mathrm{~s}$ & $450 \mathrm{~s}$ & $277 \mathrm{w}$ \\
\hline V & - & $3325 \mathrm{~m}$ & 164 & $* * 1$ & $1333 \mathrm{~s}$ & $416 \mathrm{~s}$ & $290 \mathrm{w}$ \\
\hline VI & - & $3320 \mathrm{~m} 3158 \mathrm{~m}$ & $1640 \mathrm{sh}$ & $* * 1590 \mathrm{~s}$ & $1331 \mathrm{~s}$ & $410 \mathrm{~m}$ & $273 w$ \\
\hline VII & $3401 \mathrm{w}^{*}$ & $3324 \mathrm{~m} 3168 \mathrm{~m}$ & $1674 \mathrm{mb}$ & $1643 \mathrm{mb} 1584 \mathrm{~s}$ & $1340 \mathrm{~s}$ & $418 \mathrm{~m}$ & $285 \mathrm{w}$ \\
\hline VIII & $3463 \mathrm{w}^{*}$ & $3304 \mathrm{~m} 3106 \mathrm{~m}$ & $1634 \mathrm{sh}$ & $1643 \mathrm{mb} 1584 \mathrm{~s}$ & $1340 \mathrm{~s}$ & $422 \mathrm{~m}$ & $287 \mathrm{w}$ \\
\hline
\end{tabular}

$s=$ sharp, m=medium, mb=medium broad, sh=shoulder, $w=w e a k$, "Due to phenolic $\mathrm{OH}$ of Rte and $\mathrm{R}_{2} \mathrm{Te}$ moieties; ${ }^{* *}$ band not resolved due to overlapping of band $\boldsymbol{v}_{(C=N)}$

Examination of the Schiff base (Sal-TMP) spectrum shows the presence of the hydrogen bonded conjugated- chelate ring system ${ }^{66-68}$ with its centre at $\sim 2832 \mathrm{~cm}^{-1}$. Thus intramolecular $\mathrm{H}$ - bonding is occurring by means of the formation of a quasi six- membered ring involving the $\mathrm{OH}----\mathrm{N}=\mathrm{C}$ bond.<smiles>C1=Cc2ccccc2O[NH+]=1</smiles>

Figure 1. Hydrogen bonding

Thus band disappear on chelation with aryltellurium(IV) chlorides ${ }^{69}$. Hydrogen bond contributes to planarity of the molecule which helps in chelation ${ }^{70}$. Also an intense ligand band at $1263 \mathrm{~cm}^{-1}$ (phenolic -C-O) in free ligand has shifted to higher frequency side in complexes. All these indicate that the hydroxyl group of salicylaldehyde of Schiff base is involved in coordination with tellurium ${ }^{71-73}$.

In addition, the spectra of the Schiff base shown shoulder at $1636 \mathrm{~cm}^{-1}$ with slightly resolved weak band at $1633 \mathrm{~cm}^{-1}$ and sharp band at $1593 \mathrm{~cm}^{-1}$ assigned to $v_{(\mathrm{C}=\mathrm{N})}$ mode for vibration of azomethine group ${ }^{2,4,65,74-76}$ and $v_{(\mathrm{C}=\mathrm{N}) \text { pyrim. For pyrimidine ring }}{ }^{4,77}$. These shift in aryltellurium Schiff base complexes towards higher and lower value ${ }^{74-79}$ reflecting that ligand coordinate through nitrogen atom of azomethine and pyrimidine ring.

The medium intensity band at $3317 \mathrm{~cm}^{-1}$ and $3119 \mathrm{~cm}^{-1}$ due to $v_{(\mathrm{N}-\mathrm{H})}$ asymmetric and symmetric vibrations respectively indicate the non involvement of the nitrogen atom of $\mathrm{NH}_{2}$ group attached to pyrimidine in coordination. The appearance of new weak bands around $270-295 \mathrm{~cm}^{-1}$ due to $v_{(\mathrm{Te}-\mathrm{O})}^{80-83}$ mode and medium to strong band in the range of 410-422 $\mathrm{cm}^{-1}$ due to $v_{(\mathrm{Te}-\mathrm{N})}$ mode ${ }^{84}$ further supports the involvement of phenolic oxygen (after deprotonation), azomethine and pyrimidine nitrogen atoms of Schiff base in the coordination.

Thus, IR data predict the tridentate nature of Sal-TMP involving azomethine nitrogen atom, phenolic oxygen after deprotonation and pyrimidine ring nitrogen giving rise to twosix and four membered chelate rings with the tellurium centre. 


\section{${ }^{1}$ H NMR spectra}

In order to identify the solution structure of Schiff base (Sal-TMP) and its complexes, ${ }^{1} \mathrm{H}$ NMR spectra were recorded in DMSO- $\mathrm{d}_{6}$ and given in Table 3. The proton resonance of the $\mathrm{OH}$ group at $10.92 \delta \mathrm{ppm}^{76,85}$ in Schiff base due to presence of intramolecular hydrogen bonding ${ }^{74}$ disappear on complexation indicating the involvement of phenolic oxygen in the coordination via deprotonation ${ }^{76}$. The azomethine protons which resonate as a singlet at $10.02 \delta \mathrm{ppm}$, the coordination of azomethine nitrogen to tellurium in the complexes are clearly demonstrated by a downfield shift of the peak ${ }^{86}$.

Table 3. ${ }^{1} \mathrm{H}$ NMR spectral data of Schiff base (Sal-TMP) and complexes in DMSO- $\mathrm{d}_{6}$

\begin{tabular}{|c|c|}
\hline Compound & Chemical Shift, $\delta$ ppm \\
\hline Sal-TMP & $\begin{array}{l}2.525(\mathrm{~s}, 2 \mathrm{H}, \text { methylene }), \quad 3.551-3.745\left(\mathrm{~s}, \quad 9 \mathrm{H},-\mathrm{OCH}_{3}\right), \quad 5.672(\mathrm{~s}, 1 \mathrm{H}, \\
\text { pyrimidine }), 6.508\left(\mathrm{~s}, 2 \mathrm{H},-\mathrm{NH}_{2}\right), 7.526-8.169(\mathrm{~m}, 6 \mathrm{H}, \text { aromatic proton), } \\
10.021(\mathrm{~s}, 1 \mathrm{H}, \text { azomethine }), 10.921(\mathrm{~s}, 1 \mathrm{H}, \mathrm{Schiff} \text { base } \mathrm{OH})\end{array}$ \\
\hline I & $\begin{array}{l}2.539(\mathrm{~s}, 2 \mathrm{H}, \text { methylene }), 3.598-3.840\left(\mathrm{~s}, 12 \mathrm{H},-\mathrm{OCH}_{3}\right), 6.574(\mathrm{~s}, 1 \mathrm{H}, \\
\text { pyrimidine }), 6.926\left(\mathrm{~s}, 2 \mathrm{H},-\mathrm{NH}_{2}\right), 7.383-7.522(\mathrm{~m}, 10 \mathrm{H}, \text { aromatic proton }), \\
10.209(\mathrm{~s}, 1 \mathrm{H}, \text { azomethine })\end{array}$ \\
\hline II & $\begin{array}{l}1.335\left(\mathrm{t}, 3 \mathrm{H},-\mathrm{OCH}_{2} \mathrm{CH}_{3}\right), 2.546(\mathrm{~s}, 2 \mathrm{H}, \text { methylene }), 3.963\left(\mathrm{q}, 2 \mathrm{H},-\mathrm{OCH}_{2} \mathrm{CH}_{3}\right) \text {, } \\
3.515-3.875\left(\mathrm{~s}, 9 \mathrm{H},-\mathrm{OCH}_{3}\right), 6.540(\mathrm{~s}, 1 \mathrm{H}, \text { pyrimidine }), 6.950\left(\mathrm{~s}, 2 \mathrm{H},-\mathrm{NH}_{2}\right), \\
6.993-7.809(\mathrm{~m}, 10 \mathrm{H} \text {, aromatic proton), } 10.193(\mathrm{~s}, 1 \mathrm{H} \text {, azomethine })\end{array}$ \\
\hline III & $\begin{array}{l}2.548(\mathrm{~s}, 2 \mathrm{H}, \quad \text { methylene }), \quad 3.601-3.783\left(\mathrm{~s}, 9 \mathrm{H},-\mathrm{OCH}_{3}\right), \quad 6.569(\mathrm{~s}, 1 \mathrm{H}, \\
\text { pyrimidine }), 6.914\left(\mathrm{~s}, 2 \mathrm{H},-\mathrm{NH}_{2}\right), 7.370-7.787(\mathrm{~m}, 10 \mathrm{H}, \text { aromatic proton }), \\
8.155(\mathrm{~s}, 1 \mathrm{H}, \text { phenolic } \mathrm{OH} \text { of } \mathrm{RTe}), 10.201(\mathrm{~s}, 1 \mathrm{H}, \text { azomethine })\end{array}$ \\
\hline IV & $\begin{array}{l}2.536(\mathrm{~s}, 2 \mathrm{H}, \text { methylene }), 2.527\left(\mathrm{~s}, 3 \mathrm{H},-\mathrm{CH}_{3}\right), 3.587-3.770\left(\mathrm{~s}, 9 \mathrm{H},-\mathrm{OCH}_{3}\right) \text {, } \\
\text { 6.605(s, } 1 \mathrm{H}, \text { pyrimidine }), 6.918\left(\mathrm{~s}, 2 \mathrm{H},-\mathrm{NH}_{2}\right), 7.051-7.671(\mathrm{~m}, 9 \mathrm{H} \text {, aromatic } \\
\text { proton }), 8.251(\mathrm{~s}, 1 \mathrm{H} \text {, phenolic } \mathrm{OH} \text { of } \mathrm{RTe}), 10.239(\mathrm{~s}, 1 \mathrm{H} \text {, azomethine })\end{array}$ \\
\hline V & $\begin{array}{l}2.528(\mathrm{~s}, 2 \mathrm{H}, \text { methylene }), 3.673-3.877\left(\mathrm{~s}, 15 \mathrm{H},-\mathrm{OCH}_{3}\right), 6.516(\mathrm{~s}, 1 \mathrm{H} \text {, } \\
\text { pyrimidine }), 6.882\left(\mathrm{~s}, 2 \mathrm{H},-\mathrm{NH}_{2}\right), 6.910-7.804(\mathrm{~m}, 14 \mathrm{H}, \text { aromatic proton }) \\
10.186(\mathrm{~s}, 1 \mathrm{H}, \text { azomethine })\end{array}$ \\
\hline VI & $\begin{array}{l}\left.\text { 1.363(t, } 6 \mathrm{H},-\mathrm{OCH}_{2} \mathbf{C H}_{3}\right), 2.531(\mathrm{~s}, 2 \mathrm{H}, \text { methylene }), 4.019\left(\mathrm{q}, 4 \mathrm{H},-\mathrm{OCH}_{2} \mathrm{CH}_{3}\right) \text {, } \\
3.555-3.765\left(\mathrm{~s}, 9 \mathrm{H},-\mathrm{OCH}_{3}\right), 6.510(\mathrm{~s}, 1 \mathrm{H}, \text { pyrimidine }), 6.924\left(\mathrm{~s}, 2 \mathrm{H},-\mathrm{NH}_{2}\right) \text {, } \\
\text { 7.524-7.805(m, } 14 \mathrm{H} \text {, aromatic proton }), 10.216(\mathrm{~s}, 1 \mathrm{H} \text {, azomethine })\end{array}$ \\
\hline VII & $\begin{array}{l}2.545(\mathrm{~s}, 2 \mathrm{H}, \quad \text { methylene }), 3.568-3.926\left(\mathrm{~s}, 9 \mathrm{H},-\mathrm{OCH}_{3}\right), 6.554(\mathrm{~s}, 1 \mathrm{H} \text {, } \\
\text { pyrimidine }), 6.884\left(\mathrm{~s}, 2 \mathrm{H},-\mathrm{NH}_{2}\right), 7.488-7.678(\mathrm{~m}, 14 \mathrm{H}, \text { aromatic proton), } \\
8.249\left(\mathrm{~s}, 2 \mathrm{H}, \text { phenolic } \mathrm{OH} \text { of } \mathrm{R}_{2} \mathrm{Te}\right), 10.238(\mathrm{~s}, 1 \mathrm{H}, \text { azomethine })\end{array}$ \\
\hline VIII & $\begin{array}{l}2.538(\mathrm{~s}, 2 \mathrm{H}, \text { methylene }), 2.529\left(\mathrm{~s}, 6 \mathrm{H},-\mathrm{CH}_{3}\right), 3.555-3.750\left(\mathrm{~s}, 9 \mathrm{H},-\mathrm{OCH}_{3}\right) \text {, } \\
6.502(\mathrm{~s}, 1 \mathrm{H}, \text { pyrimidine }), 6.926\left(\mathrm{~s}, 2 \mathrm{H},-\mathrm{NH}_{2}\right), 7.126-7.868(\mathrm{~m}, 12 \mathrm{H} \text {, aromatic } \\
\text { proton }), 8.149\left(\mathrm{~s}, 2 \mathrm{H} \text {, phenolic } \mathrm{OH} \text { of } \mathrm{R}_{2} \mathrm{Te}\right), 10.239(\mathrm{~s}, 1 \mathrm{H} \text {, azomethine })\end{array}$ \\
\hline
\end{tabular}
$s=$ singlet,$q=$ quartet,$t=$ triplet, $m=$ multiplet

The characterstic downfield shifting of proton signal in all complexes observed in region $5.67 \delta \mathrm{ppm}$ is due to pyrimidine proton in Schiff base clearly indicate the coordination through pyrimidine nitrogen atom ${ }^{87}$. The signal due to $-\mathrm{NH}$ proton is observed around $6.51 \delta \mathrm{ppm}$ which remain intact with slight variation in complexes is due to the proton bounded to nitrogen experience quadruple effect ${ }^{87}$. Thus salicylidene-trimethoprim act as a tridentate $-\mathrm{N},-\mathrm{N},-\mathrm{O}$ chelating ligand in Sal-TMP.ArTeCl $\mathrm{C}_{2}$ and Sal-TMP. $\mathrm{Ar}_{2} \mathrm{TeCl}$ complexes giving six coordinate tellurium having distorted octahedral geometry in these complexes as predicated from IR studies as well. The proposed structures are as given below (Figure 2). 


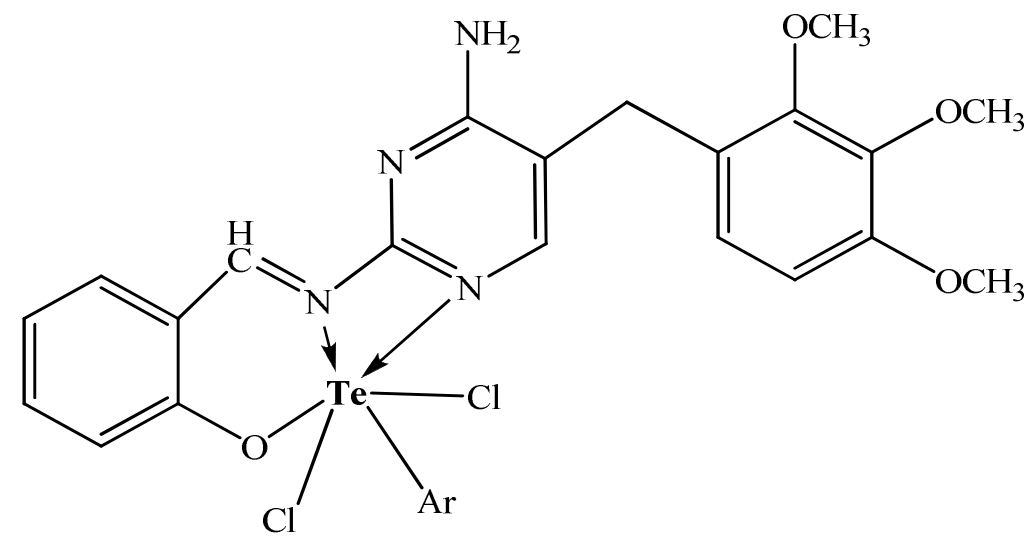<smiles></smiles>

Sal-TMP.Ar ${ }_{2} \mathrm{TeCl}$

$\mathrm{Ar}=p$-methoxyphenyl, $p$-ethoxyphenyl, $p$-hydroxyphenyl and 3-methyl-4-hydroxyphenyl

Figure 2. Proposed structures of complexes

\section{Biological Activity}

The salicylidene-trimethoprim Schiff base (Sal-TMP) and newly synthesized aryltellurium(IV) schiff base complexes were evaluated for their antimicrobial activity in vitro against Gram +ve bacteria (S. aureus ATCC 11632 and B. cereus MTCC 7350), Gram -ve bacteria (E. coli ATCC 35218, P. aeruginosa ATCC 23564, S. typhi ATCC 15499 and $P$. rettgeri DRDE) and fungal strains (A. niger, A. fumigates and A. flavus) by tube dilution method $^{88}$. Dilution of test and standard compounds were prepared double strength nutrient broth- I.P (Antibacterial) and Sabouraud Dextrose Broth -I.P (Antifungal) ${ }^{89}$. The samples were incubated at $37 \pm 1^{\circ} \mathrm{C}$ for $24 \mathrm{~h}$ (bacteria), $25 \pm 1^{\circ} \mathrm{C}$ for 7 days (A. niger), $30 \pm 1^{\circ} \mathrm{C}$ for 15 days (A. flavus), $35 \pm 1{ }^{\circ} \mathrm{C}$ for $72 \mathrm{~h}$ (A. fumigates) respectively and results were recorded in terms of MIC (The lowest concentration of test substances which inhibited values are presented in the Table 4.

The data show that the Sal-TMP complexes of aryltellurium(IV) exhibit more antimicrobial activity towards bacteria as compared to fungii. The antibacterial activity shows following trend. 
Sal-TMP.Ar $\mathrm{ArCl}_{2} \mathrm{Te}$ Sal-TMP.ArTeCl $\mathrm{Cl}_{2} \approx$ Sal-TMP Schiff base

Sal-TMP. $\mathrm{Ar}_{2} \mathrm{TeCl}$ and Sal-TMP.ArTeCl $\mathrm{Cl}_{2}$ complexes have activity towards $S$. typhi and more effectively against $B$. cereus but Schiff base does not show activity against these bacterial strains. Schiff base and its complexes show almost similar activity against fungal strains.

Table 4. Minimum Inhibitory Concentration, MIC, $\mu \mathrm{g} / \mathrm{mL}$; (-) Resistant

\begin{tabular}{|c|c|c|c|c|c|c|c|c|c|}
\hline \multirow[b]{2}{*}{ 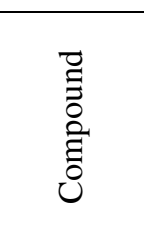 } & \multicolumn{6}{|c|}{ Bacteria strains } & \multicolumn{3}{|c|}{ Fungal strains } \\
\hline & $\begin{array}{c}S . \\
\text { aureus } \\
\text { (ATCC } \\
11632 \text { ) }\end{array}$ & $\begin{array}{l}\text { S. typhi } \\
\text { (ATCC } \\
15499 \text { ) }\end{array}$ & $\begin{array}{c}P . \\
\text { aeruginosa } \\
\text { (ATCC } \\
\text { 23564) }\end{array}$ & $\begin{array}{c}E . \\
a \quad c o l i \\
(\mathrm{ATCC} \\
35218)\end{array}$ & $\begin{array}{c}\text { B. cereus } \\
\text { (MTCC } \\
7350)\end{array}$ & $\begin{array}{c}P . \\
\text { rettgeri } \\
\text { (DRDE } \\
\text { strain) }\end{array}$ & A. niger & $\begin{array}{c}A . \\
\text { fumigates }\end{array}$ & $\begin{array}{l}\text { A. } \\
\text { flavus }\end{array}$ \\
\hline Sal-TMP & 2.5 & - & 1.25 & 5.0 & - & - & 20 & 5.0 & 1.25 \\
\hline I & 2.5 & - & 1.25 & - & 0.625 & 5.0 & - & - & - \\
\hline II & 5.0 & - & 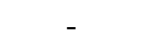 & - & 1.25 & 2.5 & 20 & 5.0 & 1.25 \\
\hline III & - & 20 & 10 & 5.0 & 1.25 & - & 5.0 & - & - \\
\hline IV & 1.25 & 2.5 & 1.25 & 5.0 & - & - & 5.0 & 10 & 5.0 \\
\hline V & - & 20 & 10 & 5.0 & 1.25 & - & 20 & - & - \\
\hline VI & 1.25 & 2.5 & 1.25 & 5.0 & - & - & - & 5.0 & - \\
\hline VII & 1.25 & - & 5.0 & 1.25 & 0.625 & 5.0 & - & 10 & - \\
\hline VIII & 2.5 & - & 1.25 & 5.0 & - & - & 20 & 5.0 & 1.25 \\
\hline
\end{tabular}

\section{Conclusion}

Aryltellurium(IV) and diaryltellurium(IV) dichlorides upon reaction with Schiff base(Sal-TMP) derived from salicylaldehyde and trimethoprim yield new complexes of tellurium(IV). The synthesized complexes were characterized by elemental analyses, conductance measurement, IR and ${ }^{1} \mathrm{H}$ NMR spectral studies. The analytical data suggest that the Sal-TMP Schiff base complexes have 1:1 stoichiometry. The Schiff bases (Sal-TMP) in these complexes functions as a uninegative tridentate ligand through azomethine nitrogen, phenolic oxygen after deprotonation and pyrimidine ring nitrogen atoms. Based on these studies, distorted octahedral geometry with two chelating rings has been assigned to these complexes. The complexes have been observed to possess substantial antimicrobial activity especially against bacteria.

\section{Acknowledgement}

The authors are grateful to M. D. University, Rohtak for providing the necessary facilities. One of the authors (Deepak) is also thankful to UGC New Delhi for providing fellowship. We also thank SAIF, Panjab University Chandigarh for providing the CHN analyses and spectral data.

\section{References}

1. Burchall J J, Corcoran J W and Hahn F E, Eds., Antibiotics, Springer, New York, NY, USA, $1975,3$.

2. Iqbal M S, Khan A H, Loothar B A and Bukhari I H, Med Chem Rev., 2009, 18, 31-42.

3. Blower P J, Аnnu Rep Prog Chem Sect A, 1999, 95, 631-655; DOI:10.1039/A808038K

4. Dhahir S A, Al-Sahib S A, Al Razaq W A and Kadhim N J, Baghdad Sci J., 2010, 7(1), 621.

5. Dr. Karl Thomae Gmb H, (Biberach an der Riss. DE), United States Patent. 4829058.

6. EL-Mossalamy E H, AL-Thabati S A, ALNomaiser F M, Commun Fac Sci Univ Ank Series B, 2005, 51(2), 21-30 
7. Sinha D, Tiwari A K, Singh S, Shukla G, Mishra P, Chandra H and Mishra A K, Eur J Med Chem., 2008, 43(1), 160-165; DOI:10.1016/j.ejmech.2007.03.022

8. Crowe A J, Smith P J and Atassi G, Chem Biol Interact., 1980, 32(1-2), 171-178; DOI:10.1016/0009-2797(80)90075-7

9. Wang M, Wang L F, Li Y Z, Li Q X, Xu Z D and Qu D M, Trans Met Chem., 2001, 26(3), 307-310; DOI:10.1023/A:1007159301849

10. Zishen Wu, Ziqi Gui and Zhenhuan Yen, Synth React Inorg Met Org Chem., 1990, 20(3), 335-344.

11. Desai S B, Desai P B and Desai K R, Hetrocycl Commun., 2001, 7(1), 83-90; DOI:10.1515/HC.2001.7.1.83

12. Dhumwad S D, Gudasi K B and Goudar T R, Indian J Chem., 1994, 33A, 320-324.

13. Przybylski P, Huczynski A, Pyta K, Brzezinski B and Bartl F, Curr Org Chem., 2009, 13(2), 124-148; DOI:10.2174/138527209787193774

14. Pandeya S N, Sriram D, Nath G and De Clercq E, Pharm Acta Hely., 1999, 74(1), 7-11.

15. Karthikeyan M S, Parsad D J, Poojary B, Bhat K S, Holla B S and Kumari N S, Bioorg. Med. Chem., 2006, 14(22), 7482-7489; DOI:10.1016/j.bmc.2006.07.015

16. Holla B S, Akberali P M and Shivananda M K, II Farmaco, 2001, 56(12), 919-927; DOI:10.1016/S0014-827X(01)01124-7

17. Jarrahpour A, Khalili D, De Clercq E, Salmi C and Brunel J M, Molecules, 2007, 12(8), 1720-1730; DOI:10.3390/12081720

18. Da Silva C M, da Silva D L, Modolo L V, Alves R B, de Resende, M A, Martins C V B and de Fatima A J, Adv Res., 2011, 2, 1-8; DOI:10.1016/j.jare.2010.05.004

19. Singh H, Yadav L D S and Mishra S B S, J Inorg Nucl Chem., 1981, 43(7), 17011704; DOI:10.1016/0022-1902(81)80367-3

20. Saravanan G, Pannerselvam P and Prakash C R, J Adv Pharm Techn Res., 2010, 1(3), 320-325; DOI:10.4103/0110-5558.72426

21. Panneerselvam P, Nair R R, Vijayalakshmi G, Subramanian E H and Sridhar S K, Eur. J. Med. Chem. 2005, 40, 225-229; DOI:10.1016/j.ejmech.2004.09.003

22. Singh N K and Singh S B, Indian J Chem., 2001, 40A, 1070-1075.

23. Walsh O M, Meegan M J, Prendergast R M, Nakib T A, Eur J Med Chem., 1996, 31, 989-1000; DOI:10.1016/S0223-5234(97)86178-8

24. Odabasoglu M, Arslan F, Ölmez H and Büyükgüngör O, Dyes and Pigments, 2007, 75(3), 507-515; DOI:10.1016/j.dyepig.2006.06.033

25. Vicini P, Geronikaki A, Incerti M, Busonera B, Poni G, Cabras C A and Colla P L, Bioorg Med Chem., 2003, 11(23), 4785-4789; DOI:10.1016/S09680896(03)00493-0

26. Pandeya S N, Sriram D, Nath G and DeClercq E, Eur J Pharm Sci., 1999, 9, 25-31.

27. Samadhiya S and Halve A, Orient J Chem., 2001, 17, 119-122.

28. Celik Ö, Ulusoy M, Tas E and Ide S, Anal Sciences, 2007, 23, x185.

29. Mahmoud M R, El-Gyar S A, Mousrafa A A and Shaker A, Polyhedron, 1987, 6(5), 1017-1020; DOI:10.1016/S0277-5387(00)80947-X

30. Qing-Yu H, Zheng-Hua M and Ya-Me Z, J Coord Chem., 1990, 21(3), 199-207; DOI:10.1080/00958979009409716

31. Cozzi P G, Chem Soc Rev., 2004, 33, 410-421; DOI:10.1039/B307853C

32. Tarafder M T H, Jin KT, Crouse K A, Ali A M. Yamin, B $M$ and Fun H K, Polyhedron, 2002, 21(25-26), 2547-2554; DOI:10.1016/S0277-5387(02)01188-9

33. Pandeya S N, Sriram D, Clercq E DE., Pannecouque C and Witvrouw M, Indian J Pharm Sci., 1998, 60(4), 207-212. 
34. Fleischman S H and Brooks C L, Proteins: Structure, Function and Genetics, 1990, 7, 52-61.

35. Suling W J, Reynolds R C, Barrow E W, Wilson L N, Piper J R and Barrow W W, J Antimicrobial Chemother., 1998, 42(6), 811-815; DOI:10.1093/jac/42.6.811

36. Gulcan M, Sonmez M and Berber I, Turkey J Chem., 2012, 36(1), 189-200.

37. Raman N, Kulandaisamy A and Jeyasubramanian K, Synth React Inorg Met Org Chem., 2001, 31(7), 1249-1270.

38. Wynne K J and Pearson P S, Inorg Chem., 1971, 10(12), 2735-2739; DOI:10.1021/ic50106a022

39. Wynne K J and Pearson P S, J Chem Soc Commun., 1970, 556-557; DOI:10.1039/C2970000556B

40. Wynne K J, Clark A J and Berg M, J Chem Soc Dalton Trans., 1972, 2370-2374; DOI:10.1039/DT9720002370

41. Clark E R, Collet A J and Naik D G, J Chem Soc Dalton Trans., 1973, 1961-1962; DOI:10.1039/DT9730001961

42. Berg M C, Diss Abstr Int., 1972, 33, 2982.

43. Srivastava T N, Singh M and Singh H B, Indian J Chem., 1982, 21A, 307-309.

44. Srivastava T N, Srivastava R C and Srivastava M, Indian J Chem., 1982, 21A, 539.

45. Srivastava T N, Srivastava R C and Srivastava V K, J Indian Chem Soc., 1983, 60, 891-892.

46. Garad M V, Polyhedron, 1985, 4(8), 1353-1355; DOI:10.1016/S0277-5387(00)86963-6

47. Verma K K and Reena, Synth React Inorg Met-Org Chem., 1999, 29(3), 499-512; DOI:10.1080/00945719909349465

48. Verma K K, Dahiya R and Soni D, Synth React Inorg Met Org Chem., 1999, 29(6), 1033-1052; DOI:10.1080/00945719909349509

49. Verma K K and Dahiya R, Synth React Inorg Met Org Chem., 1999, 29(7), 12991314; DOI:10.1080/00945719909349529

50. Verma K K and Reena, Phosphorus, Sulfur Silicon Related Elements, 1999, 148(1), 227-234; DOI:10.1080/10426509908037013

51. Verma K K and Seema, Int J Chem Sci., 2008, 6, 371-380.

52. Srivastava S, Soni D K and Gupta H S, J Indian Chem Soc., 1996, 73, 255.

53. Narwal J K, Chhabra S, Malik R K, Garg S and Verma K K, Oriental J Chem., 2013, 29, 1339-1349.

54. Chhabra S and Verma K K, J Chem Pharm Res., 2010, 2(4), 569-575.

55. Vogel A I, A Test Book of Organic Chemistry, $3^{\text {rd }}$ Edn., Longman, London, 1975.

56. Weissberger A, Ed., Technique of Organic Chemistry, Vol. 7, $2^{\text {nd }}$ Edn., Interscience Publishers, Inc. N. Y., 1967.

57. Morgan G T and Kellet R E, J Chem Soc., 1926, 1080-1088; DOI:10.1039/JR9262901080

58. Petragnani $\mathrm{N}$ and Stefani $\mathrm{H}$ A, Tellurium in Organic Chemistry, $2^{\text {nd }}$ Edn., Academic Press, London, 2007, 67, 76.

59. Bergman J, Tetrahedron, 1972, 28(12), 3323-3331; DOI:10.1016/S0040-4020(01)93674-9

60. Khandelwal B L, Kumar K and Berry F J, Inorg Chim Acta, 1981, 99(2), 135-137; DOI:10.1016/S0020-1693(00)87958-X

61. Berry F J, Kustan E H, Roshani M and Smith B C, J Organometal Chem., 1975, 99(1), 115-117; DOI:10.1016/S0022-328X(00)86367-6

62. Khandelwal B L, Kumar K and Raina K, Synth React Inorg Met Org Chem., 1981, 11, 65-78.

63. Geary W J, Coord Chem Rev., 1971, 7(1), 81-122; DOI:10.1016/S0010-8545(00)80009-0 
64. Greenwood N N, Straughan B P and Wilson A E, J Chem Soc A, 1968, 2209-2212; DOI: $10.1039 / \mathrm{J} 19680002209$

65. Srivastava K P, Singh A and Singh S K, IOSR J Appl Chem., 2014, 7(4), 16-23; DOI:10.9790/5736-07411623

66. Baker A W and Shulgin A T, J Am Chem Soc., 1959, 81(7), 1523-1529; DOI:10.1021/ja01516a001

67. Freedman H H, J Am Chem Soc., 1961, 83(13), 2900-2905; DOI:10.1021/ja01474a026

68. Flett M St C, Spectrochim Acta, 1957, 10(1), 21-37; DOI:10.1016/0371-1951(57)80160-X

69. Bellamy I J, Infrared Spectra of Complex Molecules, Chapman and Hall Ltd. London, 1975.

70. Matijević-Sosa J, Vinković M and Vikić-Topić D, Croatica Chemica Acta., 2006, 79(3), 489-495.

71. Mishra A P and Soni M, Metal Baesd Drugs, 2008, 71, 243; DOI:10.1155/2008/875410

72. Aminabhavi T M, Biradar N S and Patil C S, Inorganica Chimica Acta., 1983, 78, 107-111; DOI:10.1016/S0020-1693(00)86498-1

73. Biradar N S, Aminabhavi T M and Patil C S, Inorganica Chimica Acta., 1983, 78, 47-50; DOI:10.1016/S0020-1693(00)86487-7

74. Tumer M, Celik C, Koksal H and Serin S, Transition Metal Chem., 1999, 24(5), 525532; DOI:10.1023/A:1006982622965

75. Gwaram N S, Mohd Ali H, Khaledi H, Abdulla M A, Hamid A, Hadi A, Kawi Lin T, Ching C L and Ooi C L, Molecules, 2012, 17(5), 5952;

DOI:10.3390/molecules17055952

76. Rudzinski W E and Aminabhavi T M, Inorganica Chimica Acta., 1982, 67, 177-182; DOI:10.1016/S0020-1693(00)85061-6

77. Osowole A A, Wakil S M and Alao O K, World Appl Sci J., 2015, 33(2), 336-342; DOI:10.5829/idosi.wasj.2015.33.02.22206

78. UR-Rehman S, Faiz A and Nawaz R, J Chil Chem Soc., 2013, 58(1), 1624-1627; DOI:10.4067/S0717-97072013000100026.

79. Mohamed G G and Abd El-Wahab Z H, J Thermal Anal Calorimetry, 2003, 73(1), 347-359; DOI:10.1023/A:1025126801265

80. Verma K K, Soni D and verma, Phosphorus, Sulfur Silicon, 2000, 166(1), 231-241; DOI:10.1080/10426500008076544

81. Pant B C, McWhinnie W R and Dance N S, J Organmetal Chem., 1973, 63, 305-310; DOI:10.1016/S0022-328X(73)80043-9

82. Srivastava T N, Singh J D, Indian J. Chem., 1987, 26A, 260.

83. Chauhan S, Garg S and Verma K K, Chem Sci Trans., 2016, 5(2), 431-441; DOI:10.7598/cst2016.1193

84. Kulkarani Y D, Srivastava S, Abdi S H R and Athar M, Synth React Inorg Met Org Chem., 1985, 15(8), 1043-1059; DOI:10.1080/00945718508060634

85. Chauhan S, Garg S and Verma K K, Res J Pharm Biol Chem Sci., 2016, 7(2), 265-274.

86. Aminabhavi T M and Rudzinski W E, Inorganica Chimica Acta., 1983, 76, L131L134; DOI:10.1016/S0020-1693(00)81478-4

87. Aminabhavi T M and Rudzinski W E, Inorganica Chimica Acta., 1983, 78, 51-55; DOI:10.1016/S0020-1693(00)86488-9

88. Cappuccino J C, Sherman N, Microbiology- A Laboratory Manual, Addison Wesley, California, 1999, 263.

89. Pharmacopoeia of India, Volume 1, Controller of Publications, Ministry of Health Department, Government of India, New Delhi, 2007, 37. 


\title{
Synthesis, Spectral and Biological Studies of Some Salicylidene-Trimethoprim Schiff Base Complexes of Aryltellurium(IV)
}

\author{
DEEPAK, K. K. VERMA and SAPANA GARG* \\ Department of Chemistry, Maharshi Dayanand University, Rohtak-124001, India \\ sapanagarg1511@gmail.com
}

Received 14 February 2017 / Accepted 2 March 2017

\begin{abstract}
A novel monobasic tridentate Schiff base salicylidene-trimethoprim, Sal-TMP, synthesized from trimethoprim and salicylaldehyde, form stable complexes with aryltellurium(IV) trichlorides and diaryltellurium(IV) dichlorides of the type Sal-TMP.ArTeCl ${ }_{2}$ and $\mathrm{Sal}_{-} \mathrm{TMP}_{\mathrm{Ar}} \mathrm{AeCl}$ (where $\mathrm{Ar}=p$-methoxyphenyl, $p$-ethoxyphenyl, $p$-hydroxyphenyl and 3-methyl-4-hydroxyphenyl). These have been characterized by elemental analyses, molar conductance, IR and ${ }^{1} \mathrm{H}$ NMR spectroscopy. The spectral studies predict the bonding of ligand through phenolic oxygen of Schiff base after deprotonation, nitrogen of the azomethine group and pyrimidine nitrogen to give hexacoordinated tellurium(IV) complexes. The complexes have also been screened for their antimicrobial activities against various bacteria and fungi organisms.
\end{abstract}

Keywords: Salicylidene-trimethoprim Schiff base, Aryltellurium(IV), Diaryltellurium(IV), Antibacterial, Antifungal activities

\section{Introduction}

Trimethoprim and its derivatives are broad spectrum antimicrobial agents with anti-parasitic activity $^{1-6}$ and were first described by Roth and coworker ${ }^{5}$. Schiff bases, also known as azomethine due to presence of $-\mathrm{C}=\mathrm{N}$ - group, play important role in biological system, such as anticancer $^{7-12}$, antibacterial ${ }^{13-15}$, antiviral ${ }^{16-18}$, antifungal ${ }^{19-21}$ and other biological properties $^{22-27}$ and also have been extensively used as ligands in coordination chemistry because of their excellent donor abilities as chelating agent ${ }^{28-32}$. Medicinal chemists have reported new derivatives of trimethoprim(TMZ) ${ }^{33-35}$ including the Schiff base derived from salicylaldehyde $2-4,36,37$.

Also, aryltellurium(IV) chlorides are known ${ }^{38-54}$ to act as Lewis acids and form complexes with several N-, O- and S- donor bases. In view of this, we herein report some new complexes derived from aryltellurium(IV) trichlorides, $\mathrm{RTeCl}_{3}$ and diaryltellurium(IV) dichlorides, $\mathrm{R}_{2} \mathrm{TeCl}_{2}$ with salicylidene-trimethoprim Schiff base(Sal-TMP). 


\section{Experimental}

All preparations were carried out under dry $\mathrm{N}_{2}$ atmosphere and the solvents used were purified by standard method ${ }^{55,56}$ before use. The purity of compounds was checked by thin layer chromatography using silica gel-G (Merck). Melting points were determined in open capillary tube and are uncorrected.

Carbon, hydrogen and nitrogen analyses were obtained microanalytically from SAIF, Panjab University Chandigarh on a ThermoFinnigan CHNS analyser. Conductivity was measured in DMSO at $25 \pm 2{ }^{\circ} \mathrm{C}$ with dip type conductivity cell on a microprocessor based conductivity bridge type MICROSIL.

IR $\left(4000-400 \mathrm{~cm}^{-1}\right)$ and far IR $\left(400-50 \mathrm{~cm}^{-1}\right)$ spectra were recorded in $\mathrm{KBr} /$ polyethylene pellets on a FT-Infrared spectrophotometer model RZX (Perkin Elmer) at SAIF, Panjab University Chandigarh. Proton magnetic resonance spectra were recorded in DMSO- $\mathrm{d}_{6}$ using TMS as an internal reference on BRUKER AVANCE II 400 NMR spectrometer. The antimicrobial screening was carried out by tube dilution method at Department of Pharmaceutical Sciences, M. D. University, Rohtak, India.

\section{Preparation of aryltellurium(IV) trichlorides and diaryltellurium(IV) dichlorides}

p-Methoxyphenyltellurium(IV) $\quad$ trichloride $^{57,58}, \quad$ bis(p-methoxyphenyl)tellurium(IV) dichloride ${ }^{58,59}, \quad p$-ethoxyphenyltellurium (IV) trichloride ${ }^{60}$, bis( $p$-ethoxyphenyl)tellurium dichloride $^{60} p$-hydroxyphenyltellurium(IV) trichloride ${ }^{61}$, bis( $p$-hydroxyphenyl) tellurium(IV) dichloride $^{61}$, 3-methyl-4-hydroxyphenyltellurium(IV) trichloride $^{62}$ and bis(3-methyl-4hydroxyphenyl)tellurium(IV) dichloride ${ }^{62}$ were prepared by the reactions of $\mathrm{TeCl}_{4}$ with anisole, phenetole, phenol, $o$-cresol respectively, by the methods reported in the literature ${ }^{57-62}$.

\section{Preparation of salicylidene-trimethoprim Schiff base (Sal-TMP)}

Equimolar quantity of saturated methanolic solution of drug and salicylaldehyde were mixed thoroughly. To this mixture $0.1 \%$ methanolic $\mathrm{KOH}$ was added to adjust the $\mathrm{pH}$ of the solution between 7-8 and was refluxed for 2 hours. A clear yellowish-green coloured solution was obtained. After completion of the reaction, the Schiff base ligand was isolated by crystallization after volume reduction by evaporation. The crystalline product was filtered and dried under vacuum and kept in desiccator over $\mathrm{P}_{4} \mathrm{O}_{10}$ until further use. Yield $=$ $80 \%$, M.pt.(decomp.) $=188-190{ }^{\circ} \mathrm{C}$. Analysis (Calculated) $\mathrm{C}_{21} \mathrm{H}_{22} \mathrm{~N}_{4} \mathrm{O}_{4}: \mathrm{C}(63.95), \mathrm{H}(5.62)$ and $\mathrm{N}(14.20)$; Found: $\mathrm{C}(63.50), \mathrm{H}(5.27)$ and $\mathrm{N}(13.99)$.

\section{Preparation of salicylidene-trimethoprim complexes of aryltellurium(IV) trichlorides} and diaryltellurium(IV) dichlorides

Aryltellurium(IV) trichlorides, $\mathrm{ArTeCl}_{3}$ and diaryltellurium(IV) dichlorides $\mathrm{Ar}_{2} \mathrm{TeCl}_{2}$ (Ar= $p$-methoxyphenyl, $p$-ethoxyphenyl, $p$-hydroxyphenyl and 3-methyl-4-hydroxyphenyl), when reacted with sodium salt of salicylidene-trimethoprim in equimolar ratio, yield SalTMP.ArTeCl $\mathrm{Cl}_{2}$ and Sal-TMP.Ar $\mathrm{TeCl}_{2} \mathrm{Type}$ complexes.

Sodium salt of the ligand was prepared by reacting equimolar (1:1) quantity of sodium metal and Schiff base in methanol. The solvent was distilled off to obtain sodium salt of Schiff base. Then a methanolic saturated solution of $2 \mathrm{mmol}$ of aryltellurium(IV) trichloride or diaryltellurium(IV) dichloride was added dropwise to suspension of $2 \mathrm{mmol}$ of sodium salt of Schiff base in about $50 \mathrm{~mL}$ benzene under reflux. The reaction mixture was further refluxed for 3-4 hours, cooled and precipitated sodium chloride was filtered off. The filtrate was then concentrated to about one third of original volume under reduced pressure and cooled in an ice bath to obtain coloured product. This was filtered, washed with benzene + methanol (1:1) and dried in vacuum desiccator over $\mathrm{P}_{4} \mathrm{O}_{10}$. 


\section{Results and Discussion}

$\mathrm{TeCl}_{4}$ when heated with anisole ${ }^{57-59}$, phenetole ${ }^{60}$, phenol ${ }^{61}, o$-cresol ${ }^{62}$ (Ar-H) appears to undergo Friedel-Crafts type condensation reaction whereby $\mathrm{TeCl}_{3}{ }^{+}$unit attacks a position para to the methoxy/ethoxy/hydroxy groups in the aromatic rings, thus resulting in the formation of aryltellurium(IV) trichlorides and diaryltellurium(IV) dichlorides.

$$
\begin{gathered}
\mathrm{Ar}-\mathrm{H}+\mathrm{TeCl}_{4} \longrightarrow \mathrm{ArTeCl}_{3}+\mathrm{HCl} \\
2 \mathrm{Ar}-\mathrm{H}+\mathrm{TeCl}_{4} \longrightarrow \mathrm{Ar}_{2} \mathrm{TeCl}_{2}+2 \mathrm{HCl}
\end{gathered}
$$

Preparation of salicylidene-trimethoprim Schiff base (Sal-TMP) by the reaction of trimethoprim drug and salicylaldehyde can be represented by following equations.

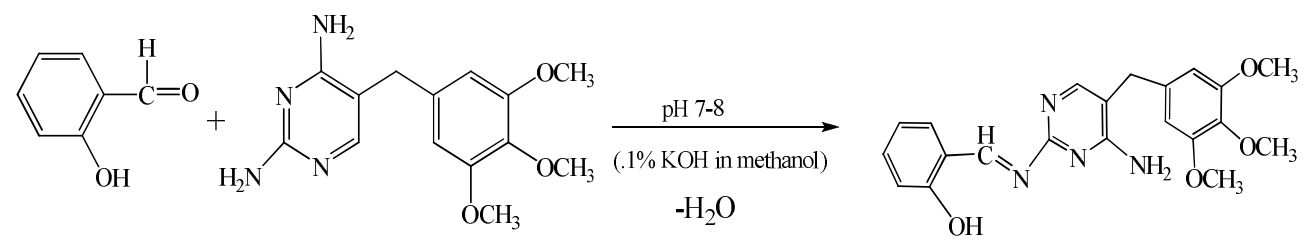

Sodium salt of salicylidene-trimethoprim Schiff base (Sal-TMP) reacts with aryltellurium(IV) trichlorides and diaryltellurium(IV) dichlorides in 1:1 molar ratio to yield the corresponding aryltellurium(IV) complexes.

$$
\begin{aligned}
& \mathrm{Sal}-\mathrm{TMP}+\mathrm{ArTeCl}_{3} \underset{\mathrm{NaCl}}{\stackrel{\mathrm{Na} / \mathrm{CH}_{3} \mathrm{OH}}{\longrightarrow}} \text { (Sal-TMP).ArTeCl } 2 \\
& \mathrm{Sal}-\mathrm{TMP}+\mathrm{Ar}_{2} \mathrm{TeCl}_{2} \underset{-\mathrm{NaCl}}{\stackrel{\mathrm{Na} / \mathrm{CH}_{3} \mathrm{OH}}{\longrightarrow}} \text { (Sal-TMP). } \mathrm{Ar}_{2} \mathrm{TeCl}_{2}
\end{aligned}
$$

All the tellurium(IV) complexes are coloured, crystalline solids, stable at room temperature and non-hygroscopic in nature. The complexes have been analysed for their tellurium, chlorine, carbon, hydrogen and nitrogen contents and the data along with their physical properties and yields are presented in Table 1.

\section{Conductance studies}

Molar conductance $\left(\Lambda_{M}\right)$ data for the complexes in DMSO are complied in Table 1. The $\Lambda_{M}$ value at $c a .10^{-3} \mathrm{M}$ for aryltellurium(IV) complexes in DMSO lie in the range 27.36-91.83 S $\mathrm{cm}^{2} \mathrm{~mol}^{-1}$ which predict the non electrolyte to $1: 1$ electrolyte $^{63,64}$ type behaviour of these complexes in DMSO, probably due to ionization into $\mathrm{ArTeCl}$.Sal- $\mathrm{TMP}^{+} / \mathrm{Ar}_{2} \mathrm{Te}$.Sal-TMP ${ }^{+}$ and $\mathrm{Cl}^{-}$in DMSO. This conductance behavoiur of tellurium(IV) salicylidene-trimethoprim Schiff base complexes is different from those of transition metal complexes ${ }^{65}$, which are reported to be non-electrolytes. The higher $\Lambda_{\mathrm{M}}$ values for some complexes may be due to steric factors and donor behaviour of DMSO to result in probable dissociation into solvated cation and $\mathrm{L}^{-}$along with $\mathrm{Cl}^{-}$in DMSO.

\section{Infrared spectra}

The IR data of Schiff base and its tellurium(IV) complexes are listed in Table 2. The spectra of Sal-TMP Schiff base complexes are quite complex and an attempt has therefore been made to identify the donor sites by comparing the spectra of complexes with parent ligand and $\mathrm{ArTeCl}_{3} / \mathrm{Ar}_{2} \mathrm{TeCl}_{2}$. 
Table 1. Analytical data, molar conductance and physical properties of salicylidene-trimethoprim Schiff base (Sal-TMP) complexes of tellurium(IV)

\begin{tabular}{|c|c|c|c|c|c|c|c|c|c|c|}
\hline \multirow[b]{2}{*}{$\begin{array}{l}\text { Compound } \\
\text { No. }\end{array}$} & \multirow[b]{2}{*}{$\begin{array}{l}\text { Complex } \\
\text { (Ar) }\end{array}$} & \multirow{2}{*}{$\begin{array}{c}\text { Empirical } \\
\text { formula } \\
\text { (Formula Wt.) }\end{array}$} & \multirow[b]{2}{*}{$\begin{array}{l}\text { Colour } \\
\text { (Yield, \%) }\end{array}$} & \multirow{2}{*}{$\begin{array}{l}\text { M. Pt. } \\
{ }^{\circ} \mathrm{C} \\
\text { dec. }\end{array}$} & \multicolumn{5}{|c|}{ Analyses \% Found (Calculated) } & \multirow{2}{*}{$\begin{array}{l}\Lambda_{\mathrm{M}} \text { at } c a \\
10^{-3} \mathrm{M} \mathrm{S} \\
\mathrm{cm}^{2} \mathrm{~mol}^{-1} \\
\text { in DMSO }\end{array}$} \\
\hline & & & & & $\mathrm{C}$ & $\mathrm{H}$ & $\mathrm{N}$ & $\mathrm{Te}$ & $\mathrm{Cl}$ & \\
\hline $\begin{array}{l}\text { Schiff } \\
\text { Base }\end{array}$ & Sal-TMP & $\mathrm{C}_{21} \mathrm{H}_{22} \mathrm{~N}_{4} \mathrm{O}_{4}$ & $\begin{array}{l}\text { Yellowish } \\
\text {-green }(80)\end{array}$ & $188-190$ & $\begin{array}{c}63.50 \\
(63.95)\end{array}$ & $\begin{array}{c}5.27 \\
(5.62)\end{array}$ & $\begin{array}{l}13.99 \\
(14.20)\end{array}$ & - & - & - \\
\hline I & $\begin{array}{l}\text { (Sal-TMP).ArTeCl }{ }_{2} \\
\text { (p-methoxyphenyl) }\end{array}$ & $\begin{array}{c}\mathrm{C}_{28} \mathrm{H}_{28} \mathrm{Cl}_{2} \mathrm{~N}_{4} \mathrm{O}_{5} \mathrm{Te} \\
(699.05)\end{array}$ & $\begin{array}{l}\text { Light cream } \\
(92)\end{array}$ & $230-232$ & $\begin{array}{c}47.88 \\
(48.11)\end{array}$ & $\begin{array}{c}3.99 \\
(4.04)\end{array}$ & $\begin{array}{c}7.75 \\
(8.01)\end{array}$ & $\begin{array}{c}18.07 \\
(18.24)\end{array}$ & $\begin{array}{c}9.89 \\
(10.01)\end{array}$ & 53.19 \\
\hline II & $\begin{array}{c}\text { (Sal-TMP).ArTeCl } \\
\text { (p-ethoxyphenyl) }\end{array}$ & $\begin{array}{c}\mathrm{C}_{29} \mathrm{H}_{30} \mathrm{Cl}_{2} \mathrm{~N}_{4} \mathrm{O}_{5} \mathrm{Te} \\
(713.08)\end{array}$ & $\begin{array}{c}\text { Cream } \\
(85)\end{array}$ & $258-260$ & $\begin{array}{c}48.12 \\
(48.85)\end{array}$ & $\begin{array}{c}3.87 \\
(4.24)\end{array}$ & $\begin{array}{l}7.52 \\
(7.86)\end{array}$ & $\begin{array}{c}17.38 \\
(17.89)\end{array}$ & $\begin{array}{c}9.52 \\
(9.81)\end{array}$ & 52.88 \\
\hline III & $\begin{array}{c}(\mathrm{Sal}-\mathrm{TMP}) \cdot \mathrm{ArTeCl}_{2} \\
(p \text {-hydroxyphenyl })\end{array}$ & $\begin{array}{c}\mathrm{C}_{27} \mathrm{H}_{26} \mathrm{Cl}_{2} \mathrm{~N}_{4} \mathrm{O}_{5} \mathrm{Te} \\
(685.03)\end{array}$ & $\begin{array}{l}\text { Dark cream } \\
\quad(94)\end{array}$ & $208-210$ & $\begin{array}{c}46.78 \\
(47.34)\end{array}$ & $\begin{array}{c}3.50 \\
(3.83)\end{array}$ & $\begin{array}{c}7.88 \\
(8.18)\end{array}$ & $\begin{array}{c}18.28 \\
(18.63)\end{array}$ & $\begin{array}{c}9.98 \\
(10.21)\end{array}$ & 55.73 \\
\hline IV & $\begin{array}{c}\text { (Sal-TMP).ArTeCl } \\
\text { (3-methyl-4- } \\
\text { hydroxyphenyl) }\end{array}$ & $\begin{array}{c}\mathrm{C}_{28} \mathrm{H}_{28} \mathrm{Cl}_{2} \mathrm{~N}_{4} \mathrm{O}_{5} \mathrm{Te} \\
(699.05)\end{array}$ & $\begin{array}{l}\text { Light } \\
\text { cream } \\
(80)\end{array}$ & $198-200$ & $\begin{array}{c}47.58 \\
(48.11)\end{array}$ & $\begin{array}{c}3.84 \\
(4.04)\end{array}$ & $\begin{array}{c}7.80 \\
(8.01)\end{array}$ & $\begin{array}{c}18.15 \\
(18.24)\end{array}$ & $\begin{array}{c}9.85 \\
(10.01)\end{array}$ & 58.68 \\
\hline $\mathrm{V}$ & $\begin{array}{c}(\mathrm{Sal}-\mathrm{TMP}) \cdot \mathrm{Ar}_{2} \mathrm{TeCl} \\
(p \text {-methoxyphenyl })\end{array}$ & $\begin{array}{c}\mathrm{C}_{35} \mathrm{H}_{35} \mathrm{ClN}_{4} \mathrm{O}_{6} \mathrm{Te} \\
(770.73)\end{array}$ & $\begin{array}{c}\text { Pale yellow } \\
\text { (84) }\end{array}$ & $130-132$ & $\begin{array}{c}53.84 \\
(54.54)\end{array}$ & $\begin{array}{c}4.31 \\
(4.58)\end{array}$ & $\begin{array}{c}7.11 \\
(7.27)\end{array}$ & $\begin{array}{c}16.43 \\
(16.56)\end{array}$ & $\begin{array}{c}4.42 \\
(4.60)\end{array}$ & 91.83 \\
\hline VI & $\begin{array}{c}\text { (Sal-TMP).Ar }{ }_{2} \mathrm{TeCl} \\
(p \text {-ethoxyphenyl) }\end{array}$ & $\begin{array}{c}\mathrm{C}_{37} \mathrm{H}_{39} \mathrm{ClN}_{4} \mathrm{O}_{6} \mathrm{Te} \\
(798.78)\end{array}$ & $\begin{array}{l}\text { Light yellow } \\
\text { (86) }\end{array}$ & $150-152$ & $\begin{array}{c}54.84 \\
(55.63)\end{array}$ & $\begin{array}{c}4.53 \\
(4.92)\end{array}$ & $\begin{array}{c}6.84 \\
(7.01)\end{array}$ & $\begin{array}{c}15.50 \\
(15.97)\end{array}$ & $\begin{array}{c}4.30 \\
(4.44)\end{array}$ & 35.90 \\
\hline VII & $\begin{array}{l}\text { (Sal-TMP).Ar } \mathrm{Ar}_{2} \mathrm{TeCl} \\
(p \text {-hydroxyphenyl) }\end{array}$ & $\begin{array}{c}\mathrm{C}_{33} \mathrm{H}_{31} \mathrm{ClN}_{4} \mathrm{O}_{6} \mathrm{Te} \\
(742.68)\end{array}$ & $\begin{array}{l}\text { Red } \\
(89)\end{array}$ & $146-148$ & $\begin{array}{c}52.84 \\
(53.37)\end{array}$ & $\begin{array}{c}4.01 \\
(4.21)\end{array}$ & $\begin{array}{c}7.27 \\
(7.54)\end{array}$ & $\begin{array}{c}16.89 \\
(17.18)\end{array}$ & $\begin{array}{c}4.52 \\
(4.77)\end{array}$ & 36.00 \\
\hline VIII & $\begin{array}{c}\text { (Sal-TMP). } \mathrm{Ar}_{2} \mathrm{TeCl} \\
\text { (3-methyl-4- } \\
\text { hydroxyphenyl) }\end{array}$ & $\begin{array}{c}\mathrm{C}_{35} \mathrm{H}_{35} \mathrm{ClN}_{4} \mathrm{O}_{6} \mathrm{Te} \\
(770.73)\end{array}$ & $\begin{array}{l}\text { Brown } \\
(78)\end{array}$ & $140-142$ & $\begin{array}{l}53.80 \\
(54.54)\end{array}$ & $\begin{array}{c}4.34 \\
(4.58)\end{array}$ & $\begin{array}{c}7.09 \\
(7.27)\end{array}$ & $\begin{array}{c}16.45 \\
(16.56)\end{array}$ & $\begin{array}{c}4.32 \\
(4.60)\end{array}$ & 27.36 \\
\hline
\end{tabular}


Table 2. Important infrared absorption bands $\left(\mathrm{cm}^{-1}\right)$ of Schiff base (Sal-TMP) and complexes

\begin{tabular}{|c|c|c|c|c|c|c|c|}
\hline Compound & $v_{(O-}$ & $v_{(1}$ & $v_{(C}$ & $v_{(C}$ & $v_{(\mathrm{C}-}$ & $v_{(\mathrm{Te}-\mathrm{N})}$ & $v_{(\mathrm{Te}}$ \\
\hline Sal-TMP & $2836 \mathrm{w}$ & $3317 \mathrm{~m} \mathrm{3119} \mathrm{m}$ & $1636 \mathrm{sh}$ & $1633 \mathrm{w} 1593 \mathrm{~s}$ & $1263 \mathrm{~s}$ & - & - \\
\hline $\bar{I}$ & - & $3323 \mathrm{~m} 3184 \mathrm{~m}$ & $1674 \mathrm{mb}$ & $1644 \mathrm{mb} 1587 \mathrm{~s}$ & $1341 \mathrm{~s}$ & $415 \mathrm{~m}$ & $288 \mathrm{w}$ \\
\hline II & - & $3323 \mathrm{~m} 3170 \mathrm{~m}$ & $1647 \mathrm{sh}$ & **1586 s & $1304 \mathrm{~s}$ & $420 \mathrm{~m}$ & $295 w$ \\
\hline III & 3405 m* & $3323 \mathrm{~m} 3150 \mathrm{~m}$ & $1674 \mathrm{mb}$ & $1641 \mathrm{mb} 1586 \mathrm{~s}$ & $1341 \mathrm{~s}$ & $419 \mathrm{~s}$ & $270 \mathrm{w}$ \\
\hline IV & 3398 m* & $3319 \mathrm{~m} 3172 \mathrm{~m}$ & $1652 \mathrm{mb}$ & 1649 & $1333 \mathrm{~s}$ & $450 \mathrm{~s}$ & $277 \mathrm{w}$ \\
\hline V & - & $3325 \mathrm{~m}$ & 164 & $* * 1$ & $1333 \mathrm{~s}$ & $416 \mathrm{~s}$ & $290 \mathrm{w}$ \\
\hline VI & - & $3320 \mathrm{~m} 3158 \mathrm{~m}$ & $1640 \mathrm{sh}$ & $* * 1590 \mathrm{~s}$ & $1331 \mathrm{~s}$ & $410 \mathrm{~m}$ & $273 w$ \\
\hline VII & $3401 \mathrm{w}^{*}$ & $3324 \mathrm{~m} 3168 \mathrm{~m}$ & $1674 \mathrm{mb}$ & $1643 \mathrm{mb} 1584 \mathrm{~s}$ & $1340 \mathrm{~s}$ & $418 \mathrm{~m}$ & $285 \mathrm{w}$ \\
\hline VIII & $3463 \mathrm{w}^{*}$ & $3304 \mathrm{~m} 3106 \mathrm{~m}$ & $1634 \mathrm{sh}$ & $1643 \mathrm{mb} 1584 \mathrm{~s}$ & $1340 \mathrm{~s}$ & $422 \mathrm{~m}$ & $287 \mathrm{w}$ \\
\hline
\end{tabular}

$s=$ sharp, m=medium, mb=medium broad, sh=shoulder, $w=w e a k$, "Due to phenolic $\mathrm{OH}$ of Rte and $\mathrm{R}_{2} \mathrm{Te}$ moieties; ${ }^{* *}$ band not resolved due to overlapping of band $\boldsymbol{v}_{(C=N)}$

Examination of the Schiff base (Sal-TMP) spectrum shows the presence of the hydrogen bonded conjugated- chelate ring system ${ }^{66-68}$ with its centre at $\sim 2832 \mathrm{~cm}^{-1}$. Thus intramolecular $\mathrm{H}$ - bonding is occurring by means of the formation of a quasi six- membered ring involving the $\mathrm{OH}----\mathrm{N}=\mathrm{C}$ bond.<smiles>C1=Cc2ccccc2O[NH+]=1</smiles>

Figure 1. Hydrogen bonding

Thus band disappear on chelation with aryltellurium(IV) chlorides ${ }^{69}$. Hydrogen bond contributes to planarity of the molecule which helps in chelation ${ }^{70}$. Also an intense ligand band at $1263 \mathrm{~cm}^{-1}$ (phenolic -C-O) in free ligand has shifted to higher frequency side in complexes. All these indicate that the hydroxyl group of salicylaldehyde of Schiff base is involved in coordination with tellurium ${ }^{71-73}$.

In addition, the spectra of the Schiff base shown shoulder at $1636 \mathrm{~cm}^{-1}$ with slightly resolved weak band at $1633 \mathrm{~cm}^{-1}$ and sharp band at $1593 \mathrm{~cm}^{-1}$ assigned to $v_{(\mathrm{C}=\mathrm{N})}$ mode for vibration of azomethine group ${ }^{2,4,65,74-76}$ and $v_{(\mathrm{C}=\mathrm{N}) \text { pyrim. For pyrimidine ring }}{ }^{4,77}$. These shift in aryltellurium Schiff base complexes towards higher and lower value ${ }^{74-79}$ reflecting that ligand coordinate through nitrogen atom of azomethine and pyrimidine ring.

The medium intensity band at $3317 \mathrm{~cm}^{-1}$ and $3119 \mathrm{~cm}^{-1}$ due to $v_{(\mathrm{N}-\mathrm{H})}$ asymmetric and symmetric vibrations respectively indicate the non involvement of the nitrogen atom of $\mathrm{NH}_{2}$ group attached to pyrimidine in coordination. The appearance of new weak bands around $270-295 \mathrm{~cm}^{-1}$ due to $v_{(\mathrm{Te}-\mathrm{O})}^{80-83}$ mode and medium to strong band in the range of 410-422 $\mathrm{cm}^{-1}$ due to $v_{(\mathrm{Te}-\mathrm{N})}$ mode ${ }^{84}$ further supports the involvement of phenolic oxygen (after deprotonation), azomethine and pyrimidine nitrogen atoms of Schiff base in the coordination.

Thus, IR data predict the tridentate nature of Sal-TMP involving azomethine nitrogen atom, phenolic oxygen after deprotonation and pyrimidine ring nitrogen giving rise to twosix and four membered chelate rings with the tellurium centre. 


\section{${ }^{1}$ H NMR spectra}

In order to identify the solution structure of Schiff base (Sal-TMP) and its complexes, ${ }^{1} \mathrm{H}$ NMR spectra were recorded in DMSO- $\mathrm{d}_{6}$ and given in Table 3. The proton resonance of the $\mathrm{OH}$ group at $10.92 \delta \mathrm{ppm}^{76,85}$ in Schiff base due to presence of intramolecular hydrogen bonding ${ }^{74}$ disappear on complexation indicating the involvement of phenolic oxygen in the coordination via deprotonation ${ }^{76}$. The azomethine protons which resonate as a singlet at $10.02 \delta \mathrm{ppm}$, the coordination of azomethine nitrogen to tellurium in the complexes are clearly demonstrated by a downfield shift of the peak ${ }^{86}$.

Table 3. ${ }^{1} \mathrm{H}$ NMR spectral data of Schiff base (Sal-TMP) and complexes in DMSO- $\mathrm{d}_{6}$

\begin{tabular}{|c|c|}
\hline Compound & Chemical Shift, $\delta$ ppm \\
\hline Sal-TMP & $\begin{array}{l}2.525(\mathrm{~s}, 2 \mathrm{H}, \text { methylene }), \quad 3.551-3.745\left(\mathrm{~s}, \quad 9 \mathrm{H},-\mathrm{OCH}_{3}\right), \quad 5.672(\mathrm{~s}, 1 \mathrm{H}, \\
\text { pyrimidine }), 6.508\left(\mathrm{~s}, 2 \mathrm{H},-\mathrm{NH}_{2}\right), 7.526-8.169(\mathrm{~m}, 6 \mathrm{H}, \text { aromatic proton), } \\
10.021(\mathrm{~s}, 1 \mathrm{H}, \text { azomethine }), 10.921(\mathrm{~s}, 1 \mathrm{H}, \mathrm{Schiff} \text { base } \mathrm{OH})\end{array}$ \\
\hline I & $\begin{array}{l}2.539(\mathrm{~s}, 2 \mathrm{H}, \text { methylene }), 3.598-3.840\left(\mathrm{~s}, 12 \mathrm{H},-\mathrm{OCH}_{3}\right), 6.574(\mathrm{~s}, 1 \mathrm{H}, \\
\text { pyrimidine }), 6.926\left(\mathrm{~s}, 2 \mathrm{H},-\mathrm{NH}_{2}\right), 7.383-7.522(\mathrm{~m}, 10 \mathrm{H}, \text { aromatic proton }), \\
10.209(\mathrm{~s}, 1 \mathrm{H}, \text { azomethine })\end{array}$ \\
\hline II & $\begin{array}{l}1.335\left(\mathrm{t}, 3 \mathrm{H},-\mathrm{OCH}_{2} \mathrm{CH}_{3}\right), 2.546(\mathrm{~s}, 2 \mathrm{H}, \text { methylene }), 3.963\left(\mathrm{q}, 2 \mathrm{H},-\mathrm{OCH}_{2} \mathrm{CH}_{3}\right) \text {, } \\
3.515-3.875\left(\mathrm{~s}, 9 \mathrm{H},-\mathrm{OCH}_{3}\right), 6.540(\mathrm{~s}, 1 \mathrm{H}, \text { pyrimidine }), 6.950\left(\mathrm{~s}, 2 \mathrm{H},-\mathrm{NH}_{2}\right), \\
6.993-7.809(\mathrm{~m}, 10 \mathrm{H} \text {, aromatic proton), } 10.193(\mathrm{~s}, 1 \mathrm{H} \text {, azomethine })\end{array}$ \\
\hline III & $\begin{array}{l}2.548(\mathrm{~s}, 2 \mathrm{H}, \quad \text { methylene }), \quad 3.601-3.783\left(\mathrm{~s}, 9 \mathrm{H},-\mathrm{OCH}_{3}\right), \quad 6.569(\mathrm{~s}, 1 \mathrm{H}, \\
\text { pyrimidine }), 6.914\left(\mathrm{~s}, 2 \mathrm{H},-\mathrm{NH}_{2}\right), 7.370-7.787(\mathrm{~m}, 10 \mathrm{H}, \text { aromatic proton }), \\
8.155(\mathrm{~s}, 1 \mathrm{H}, \text { phenolic } \mathrm{OH} \text { of } \mathrm{RTe}), 10.201(\mathrm{~s}, 1 \mathrm{H}, \text { azomethine })\end{array}$ \\
\hline IV & $\begin{array}{l}2.536(\mathrm{~s}, 2 \mathrm{H}, \text { methylene }), 2.527\left(\mathrm{~s}, 3 \mathrm{H},-\mathrm{CH}_{3}\right), 3.587-3.770\left(\mathrm{~s}, 9 \mathrm{H},-\mathrm{OCH}_{3}\right) \text {, } \\
\text { 6.605(s, } 1 \mathrm{H}, \text { pyrimidine }), 6.918\left(\mathrm{~s}, 2 \mathrm{H},-\mathrm{NH}_{2}\right), 7.051-7.671(\mathrm{~m}, 9 \mathrm{H} \text {, aromatic } \\
\text { proton }), 8.251(\mathrm{~s}, 1 \mathrm{H} \text {, phenolic } \mathrm{OH} \text { of } \mathrm{RTe}), 10.239(\mathrm{~s}, 1 \mathrm{H} \text {, azomethine })\end{array}$ \\
\hline V & $\begin{array}{l}2.528(\mathrm{~s}, 2 \mathrm{H}, \text { methylene }), 3.673-3.877\left(\mathrm{~s}, 15 \mathrm{H},-\mathrm{OCH}_{3}\right), 6.516(\mathrm{~s}, 1 \mathrm{H} \text {, } \\
\text { pyrimidine }), 6.882\left(\mathrm{~s}, 2 \mathrm{H},-\mathrm{NH}_{2}\right), 6.910-7.804(\mathrm{~m}, 14 \mathrm{H}, \text { aromatic proton }) \\
10.186(\mathrm{~s}, 1 \mathrm{H}, \text { azomethine })\end{array}$ \\
\hline VI & $\begin{array}{l}\left.\text { 1.363(t, } 6 \mathrm{H},-\mathrm{OCH}_{2} \mathbf{C H}_{3}\right), 2.531(\mathrm{~s}, 2 \mathrm{H}, \text { methylene }), 4.019\left(\mathrm{q}, 4 \mathrm{H},-\mathrm{OCH}_{2} \mathrm{CH}_{3}\right) \text {, } \\
3.555-3.765\left(\mathrm{~s}, 9 \mathrm{H},-\mathrm{OCH}_{3}\right), 6.510(\mathrm{~s}, 1 \mathrm{H}, \text { pyrimidine }), 6.924\left(\mathrm{~s}, 2 \mathrm{H},-\mathrm{NH}_{2}\right) \text {, } \\
\text { 7.524-7.805(m, } 14 \mathrm{H} \text {, aromatic proton }), 10.216(\mathrm{~s}, 1 \mathrm{H} \text {, azomethine })\end{array}$ \\
\hline VII & $\begin{array}{l}2.545(\mathrm{~s}, 2 \mathrm{H}, \quad \text { methylene }), 3.568-3.926\left(\mathrm{~s}, 9 \mathrm{H},-\mathrm{OCH}_{3}\right), 6.554(\mathrm{~s}, 1 \mathrm{H} \text {, } \\
\text { pyrimidine }), 6.884\left(\mathrm{~s}, 2 \mathrm{H},-\mathrm{NH}_{2}\right), 7.488-7.678(\mathrm{~m}, 14 \mathrm{H}, \text { aromatic proton), } \\
8.249\left(\mathrm{~s}, 2 \mathrm{H}, \text { phenolic } \mathrm{OH} \text { of } \mathrm{R}_{2} \mathrm{Te}\right), 10.238(\mathrm{~s}, 1 \mathrm{H}, \text { azomethine })\end{array}$ \\
\hline VIII & $\begin{array}{l}2.538(\mathrm{~s}, 2 \mathrm{H}, \text { methylene }), 2.529\left(\mathrm{~s}, 6 \mathrm{H},-\mathrm{CH}_{3}\right), 3.555-3.750\left(\mathrm{~s}, 9 \mathrm{H},-\mathrm{OCH}_{3}\right) \text {, } \\
6.502(\mathrm{~s}, 1 \mathrm{H}, \text { pyrimidine }), 6.926\left(\mathrm{~s}, 2 \mathrm{H},-\mathrm{NH}_{2}\right), 7.126-7.868(\mathrm{~m}, 12 \mathrm{H} \text {, aromatic } \\
\text { proton }), 8.149\left(\mathrm{~s}, 2 \mathrm{H} \text {, phenolic } \mathrm{OH} \text { of } \mathrm{R}_{2} \mathrm{Te}\right), 10.239(\mathrm{~s}, 1 \mathrm{H} \text {, azomethine })\end{array}$ \\
\hline
\end{tabular}
$s=$ singlet,$q=$ quartet,$t=$ triplet, $m=$ multiplet

The characterstic downfield shifting of proton signal in all complexes observed in region $5.67 \delta \mathrm{ppm}$ is due to pyrimidine proton in Schiff base clearly indicate the coordination through pyrimidine nitrogen atom ${ }^{87}$. The signal due to $-\mathrm{NH}$ proton is observed around $6.51 \delta \mathrm{ppm}$ which remain intact with slight variation in complexes is due to the proton bounded to nitrogen experience quadruple effect ${ }^{87}$. Thus salicylidene-trimethoprim act as a tridentate $-\mathrm{N},-\mathrm{N},-\mathrm{O}$ chelating ligand in Sal-TMP.ArTeCl $\mathrm{C}_{2}$ and Sal-TMP. $\mathrm{Ar}_{2} \mathrm{TeCl}$ complexes giving six coordinate tellurium having distorted octahedral geometry in these complexes as predicated from IR studies as well. The proposed structures are as given below (Figure 2). 


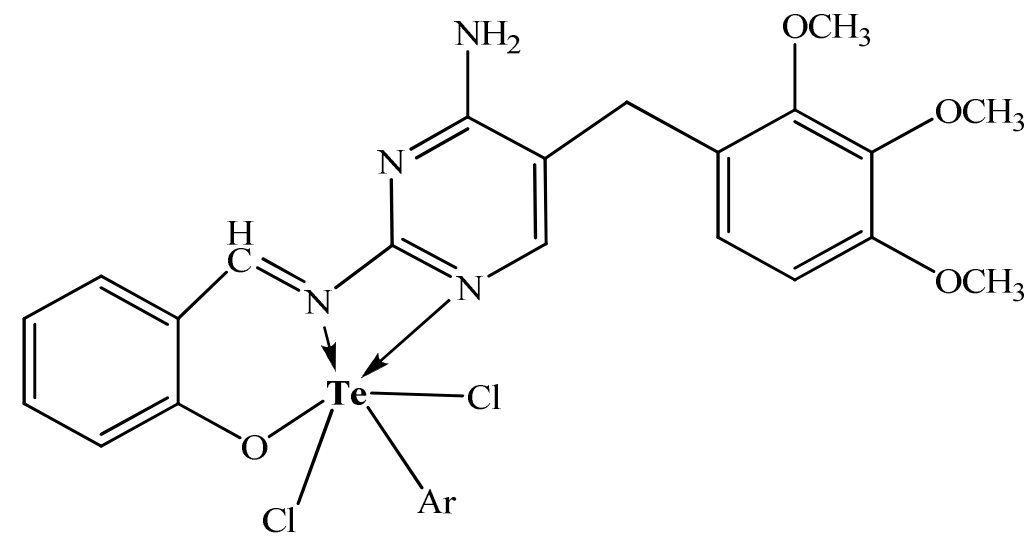<smiles></smiles>

Sal-TMP.Ar ${ }_{2} \mathrm{TeCl}$

$\mathrm{Ar}=p$-methoxyphenyl, $p$-ethoxyphenyl, $p$-hydroxyphenyl and 3-methyl-4-hydroxyphenyl

Figure 2. Proposed structures of complexes

\section{Biological Activity}

The salicylidene-trimethoprim Schiff base (Sal-TMP) and newly synthesized aryltellurium(IV) schiff base complexes were evaluated for their antimicrobial activity in vitro against Gram +ve bacteria (S. aureus ATCC 11632 and B. cereus MTCC 7350), Gram -ve bacteria (E. coli ATCC 35218, P. aeruginosa ATCC 23564, S. typhi ATCC 15499 and $P$. rettgeri DRDE) and fungal strains (A. niger, A. fumigates and A. flavus) by tube dilution method $^{88}$. Dilution of test and standard compounds were prepared double strength nutrient broth- I.P (Antibacterial) and Sabouraud Dextrose Broth -I.P (Antifungal) ${ }^{89}$. The samples were incubated at $37 \pm 1^{\circ} \mathrm{C}$ for $24 \mathrm{~h}$ (bacteria), $25 \pm 1^{\circ} \mathrm{C}$ for 7 days (A. niger), $30 \pm 1^{\circ} \mathrm{C}$ for 15 days (A. flavus), $35 \pm 1{ }^{\circ} \mathrm{C}$ for $72 \mathrm{~h}$ (A. fumigates) respectively and results were recorded in terms of MIC (The lowest concentration of test substances which inhibited values are presented in the Table 4.

The data show that the Sal-TMP complexes of aryltellurium(IV) exhibit more antimicrobial activity towards bacteria as compared to fungii. The antibacterial activity shows following trend. 
Sal-TMP.Ar $\mathrm{ArCl}_{2} \mathrm{Te}$ Sal-TMP.ArTeCl $\mathrm{Cl}_{2} \approx$ Sal-TMP Schiff base

Sal-TMP. $\mathrm{Ar}_{2} \mathrm{TeCl}$ and Sal-TMP.ArTeCl $\mathrm{Cl}_{2}$ complexes have activity towards $S$. typhi and more effectively against $B$. cereus but Schiff base does not show activity against these bacterial strains. Schiff base and its complexes show almost similar activity against fungal strains.

Table 4. Minimum Inhibitory Concentration, MIC, $\mu \mathrm{g} / \mathrm{mL}$; (-) Resistant

\begin{tabular}{|c|c|c|c|c|c|c|c|c|c|}
\hline \multirow[b]{2}{*}{ 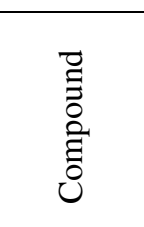 } & \multicolumn{6}{|c|}{ Bacteria strains } & \multicolumn{3}{|c|}{ Fungal strains } \\
\hline & $\begin{array}{c}S . \\
\text { aureus } \\
\text { (ATCC } \\
11632 \text { ) }\end{array}$ & $\begin{array}{l}\text { S. typhi } \\
\text { (ATCC } \\
15499 \text { ) }\end{array}$ & $\begin{array}{c}P . \\
\text { aeruginosa } \\
\text { (ATCC } \\
\text { 23564) }\end{array}$ & $\begin{array}{c}E . \\
a \quad c o l i \\
(\mathrm{ATCC} \\
35218)\end{array}$ & $\begin{array}{c}\text { B. cereus } \\
\text { (MTCC } \\
7350)\end{array}$ & $\begin{array}{c}P . \\
\text { rettgeri } \\
\text { (DRDE } \\
\text { strain) }\end{array}$ & A. niger & $\begin{array}{c}A . \\
\text { fumigates }\end{array}$ & $\begin{array}{l}\text { A. } \\
\text { flavus }\end{array}$ \\
\hline Sal-TMP & 2.5 & - & 1.25 & 5.0 & - & - & 20 & 5.0 & 1.25 \\
\hline I & 2.5 & - & 1.25 & - & 0.625 & 5.0 & - & - & - \\
\hline II & 5.0 & - & 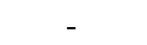 & - & 1.25 & 2.5 & 20 & 5.0 & 1.25 \\
\hline III & - & 20 & 10 & 5.0 & 1.25 & - & 5.0 & - & - \\
\hline IV & 1.25 & 2.5 & 1.25 & 5.0 & - & - & 5.0 & 10 & 5.0 \\
\hline V & - & 20 & 10 & 5.0 & 1.25 & - & 20 & - & - \\
\hline VI & 1.25 & 2.5 & 1.25 & 5.0 & - & - & - & 5.0 & - \\
\hline VII & 1.25 & - & 5.0 & 1.25 & 0.625 & 5.0 & - & 10 & - \\
\hline VIII & 2.5 & - & 1.25 & 5.0 & - & - & 20 & 5.0 & 1.25 \\
\hline
\end{tabular}

\section{Conclusion}

Aryltellurium(IV) and diaryltellurium(IV) dichlorides upon reaction with Schiff base(Sal-TMP) derived from salicylaldehyde and trimethoprim yield new complexes of tellurium(IV). The synthesized complexes were characterized by elemental analyses, conductance measurement, IR and ${ }^{1} \mathrm{H}$ NMR spectral studies. The analytical data suggest that the Sal-TMP Schiff base complexes have 1:1 stoichiometry. The Schiff bases (Sal-TMP) in these complexes functions as a uninegative tridentate ligand through azomethine nitrogen, phenolic oxygen after deprotonation and pyrimidine ring nitrogen atoms. Based on these studies, distorted octahedral geometry with two chelating rings has been assigned to these complexes. The complexes have been observed to possess substantial antimicrobial activity especially against bacteria.

\section{Acknowledgement}

The authors are grateful to M. D. University, Rohtak for providing the necessary facilities. One of the authors (Deepak) is also thankful to UGC New Delhi for providing fellowship. We also thank SAIF, Panjab University Chandigarh for providing the CHN analyses and spectral data.

\section{References}

1. Burchall J J, Corcoran J W and Hahn F E, Eds., Antibiotics, Springer, New York, NY, USA, $1975,3$.

2. Iqbal M S, Khan A H, Loothar B A and Bukhari I H, Med Chem Rev., 2009, 18, 31-42.

3. Blower P J, Аnnu Rep Prog Chem Sect A, 1999, 95, 631-655; DOI:10.1039/A808038K

4. Dhahir S A, Al-Sahib S A, Al Razaq W A and Kadhim N J, Baghdad Sci J., 2010, 7(1), 621.

5. Dr. Karl Thomae Gmb H, (Biberach an der Riss. DE), United States Patent. 4829058.

6. EL-Mossalamy E H, AL-Thabati S A, ALNomaiser F M, Commun Fac Sci Univ Ank Series B, 2005, 51(2), 21-30 
7. Sinha D, Tiwari A K, Singh S, Shukla G, Mishra P, Chandra H and Mishra A K, Eur J Med Chem., 2008, 43(1), 160-165; DOI:10.1016/j.ejmech.2007.03.022

8. Crowe A J, Smith P J and Atassi G, Chem Biol Interact., 1980, 32(1-2), 171-178; DOI:10.1016/0009-2797(80)90075-7

9. Wang M, Wang L F, Li Y Z, Li Q X, Xu Z D and Qu D M, Trans Met Chem., 2001, 26(3), 307-310; DOI:10.1023/A:1007159301849

10. Zishen Wu, Ziqi Gui and Zhenhuan Yen, Synth React Inorg Met Org Chem., 1990, 20(3), 335-344.

11. Desai S B, Desai P B and Desai K R, Hetrocycl Commun., 2001, 7(1), 83-90; DOI:10.1515/HC.2001.7.1.83

12. Dhumwad S D, Gudasi K B and Goudar T R, Indian J Chem., 1994, 33A, 320-324.

13. Przybylski P, Huczynski A, Pyta K, Brzezinski B and Bartl F, Curr Org Chem., 2009, 13(2), 124-148; DOI:10.2174/138527209787193774

14. Pandeya S N, Sriram D, Nath G and De Clercq E, Pharm Acta Hely., 1999, 74(1), 7-11.

15. Karthikeyan M S, Parsad D J, Poojary B, Bhat K S, Holla B S and Kumari N S, Bioorg. Med. Chem., 2006, 14(22), 7482-7489; DOI:10.1016/j.bmc.2006.07.015

16. Holla B S, Akberali P M and Shivananda M K, II Farmaco, 2001, 56(12), 919-927; DOI:10.1016/S0014-827X(01)01124-7

17. Jarrahpour A, Khalili D, De Clercq E, Salmi C and Brunel J M, Molecules, 2007, 12(8), 1720-1730; DOI:10.3390/12081720

18. Da Silva C M, da Silva D L, Modolo L V, Alves R B, de Resende, M A, Martins C V B and de Fatima A J, Adv Res., 2011, 2, 1-8; DOI:10.1016/j.jare.2010.05.004

19. Singh H, Yadav L D S and Mishra S B S, J Inorg Nucl Chem., 1981, 43(7), 17011704; DOI:10.1016/0022-1902(81)80367-3

20. Saravanan G, Pannerselvam P and Prakash C R, J Adv Pharm Techn Res., 2010, 1(3), 320-325; DOI:10.4103/0110-5558.72426

21. Panneerselvam P, Nair R R, Vijayalakshmi G, Subramanian E H and Sridhar S K, Eur. J. Med. Chem. 2005, 40, 225-229; DOI:10.1016/j.ejmech.2004.09.003

22. Singh N K and Singh S B, Indian J Chem., 2001, 40A, 1070-1075.

23. Walsh O M, Meegan M J, Prendergast R M, Nakib T A, Eur J Med Chem., 1996, 31, 989-1000; DOI:10.1016/S0223-5234(97)86178-8

24. Odabasoglu M, Arslan F, Ölmez H and Büyükgüngör O, Dyes and Pigments, 2007, 75(3), 507-515; DOI:10.1016/j.dyepig.2006.06.033

25. Vicini P, Geronikaki A, Incerti M, Busonera B, Poni G, Cabras C A and Colla P L, Bioorg Med Chem., 2003, 11(23), 4785-4789; DOI:10.1016/S09680896(03)00493-0

26. Pandeya S N, Sriram D, Nath G and DeClercq E, Eur J Pharm Sci., 1999, 9, 25-31.

27. Samadhiya S and Halve A, Orient J Chem., 2001, 17, 119-122.

28. Celik Ö, Ulusoy M, Tas E and Ide S, Anal Sciences, 2007, 23, x185.

29. Mahmoud M R, El-Gyar S A, Mousrafa A A and Shaker A, Polyhedron, 1987, 6(5), 1017-1020; DOI:10.1016/S0277-5387(00)80947-X

30. Qing-Yu H, Zheng-Hua M and Ya-Me Z, J Coord Chem., 1990, 21(3), 199-207; DOI:10.1080/00958979009409716

31. Cozzi P G, Chem Soc Rev., 2004, 33, 410-421; DOI:10.1039/B307853C

32. Tarafder M T H, Jin KT, Crouse K A, Ali A M. Yamin, B $M$ and Fun H K, Polyhedron, 2002, 21(25-26), 2547-2554; DOI:10.1016/S0277-5387(02)01188-9

33. Pandeya S N, Sriram D, Clercq E DE., Pannecouque C and Witvrouw M, Indian J Pharm Sci., 1998, 60(4), 207-212. 
34. Fleischman S H and Brooks C L, Proteins: Structure, Function and Genetics, 1990, 7, 52-61.

35. Suling W J, Reynolds R C, Barrow E W, Wilson L N, Piper J R and Barrow W W, J Antimicrobial Chemother., 1998, 42(6), 811-815; DOI:10.1093/jac/42.6.811

36. Gulcan M, Sonmez M and Berber I, Turkey J Chem., 2012, 36(1), 189-200.

37. Raman N, Kulandaisamy A and Jeyasubramanian K, Synth React Inorg Met Org Chem., 2001, 31(7), 1249-1270.

38. Wynne K J and Pearson P S, Inorg Chem., 1971, 10(12), 2735-2739; DOI:10.1021/ic50106a022

39. Wynne K J and Pearson P S, J Chem Soc Commun., 1970, 556-557; DOI:10.1039/C2970000556B

40. Wynne K J, Clark A J and Berg M, J Chem Soc Dalton Trans., 1972, 2370-2374; DOI:10.1039/DT9720002370

41. Clark E R, Collet A J and Naik D G, J Chem Soc Dalton Trans., 1973, 1961-1962; DOI:10.1039/DT9730001961

42. Berg M C, Diss Abstr Int., 1972, 33, 2982.

43. Srivastava T N, Singh M and Singh H B, Indian J Chem., 1982, 21A, 307-309.

44. Srivastava T N, Srivastava R C and Srivastava M, Indian J Chem., 1982, 21A, 539.

45. Srivastava T N, Srivastava R C and Srivastava V K, J Indian Chem Soc., 1983, 60, 891-892.

46. Garad M V, Polyhedron, 1985, 4(8), 1353-1355; DOI:10.1016/S0277-5387(00)86963-6

47. Verma K K and Reena, Synth React Inorg Met-Org Chem., 1999, 29(3), 499-512; DOI:10.1080/00945719909349465

48. Verma K K, Dahiya R and Soni D, Synth React Inorg Met Org Chem., 1999, 29(6), 1033-1052; DOI:10.1080/00945719909349509

49. Verma K K and Dahiya R, Synth React Inorg Met Org Chem., 1999, 29(7), 12991314; DOI:10.1080/00945719909349529

50. Verma K K and Reena, Phosphorus, Sulfur Silicon Related Elements, 1999, 148(1), 227-234; DOI:10.1080/10426509908037013

51. Verma K K and Seema, Int J Chem Sci., 2008, 6, 371-380.

52. Srivastava S, Soni D K and Gupta H S, J Indian Chem Soc., 1996, 73, 255.

53. Narwal J K, Chhabra S, Malik R K, Garg S and Verma K K, Oriental J Chem., 2013, 29, 1339-1349.

54. Chhabra S and Verma K K, J Chem Pharm Res., 2010, 2(4), 569-575.

55. Vogel A I, A Test Book of Organic Chemistry, $3^{\text {rd }}$ Edn., Longman, London, 1975.

56. Weissberger A, Ed., Technique of Organic Chemistry, Vol. 7, $2^{\text {nd }}$ Edn., Interscience Publishers, Inc. N. Y., 1967.

57. Morgan G T and Kellet R E, J Chem Soc., 1926, 1080-1088; DOI:10.1039/JR9262901080

58. Petragnani $\mathrm{N}$ and Stefani $\mathrm{H}$ A, Tellurium in Organic Chemistry, $2^{\text {nd }}$ Edn., Academic Press, London, 2007, 67, 76.

59. Bergman J, Tetrahedron, 1972, 28(12), 3323-3331; DOI:10.1016/S0040-4020(01)93674-9

60. Khandelwal B L, Kumar K and Berry F J, Inorg Chim Acta, 1981, 99(2), 135-137; DOI:10.1016/S0020-1693(00)87958-X

61. Berry F J, Kustan E H, Roshani M and Smith B C, J Organometal Chem., 1975, 99(1), 115-117; DOI:10.1016/S0022-328X(00)86367-6

62. Khandelwal B L, Kumar K and Raina K, Synth React Inorg Met Org Chem., 1981, 11, 65-78.

63. Geary W J, Coord Chem Rev., 1971, 7(1), 81-122; DOI:10.1016/S0010-8545(00)80009-0 
64. Greenwood N N, Straughan B P and Wilson A E, J Chem Soc A, 1968, 2209-2212; DOI: $10.1039 / \mathrm{J} 19680002209$

65. Srivastava K P, Singh A and Singh S K, IOSR J Appl Chem., 2014, 7(4), 16-23; DOI:10.9790/5736-07411623

66. Baker A W and Shulgin A T, J Am Chem Soc., 1959, 81(7), 1523-1529; DOI:10.1021/ja01516a001

67. Freedman H H, J Am Chem Soc., 1961, 83(13), 2900-2905; DOI:10.1021/ja01474a026

68. Flett M St C, Spectrochim Acta, 1957, 10(1), 21-37; DOI:10.1016/0371-1951(57)80160-X

69. Bellamy I J, Infrared Spectra of Complex Molecules, Chapman and Hall Ltd. London, 1975.

70. Matijević-Sosa J, Vinković M and Vikić-Topić D, Croatica Chemica Acta., 2006, 79(3), 489-495.

71. Mishra A P and Soni M, Metal Baesd Drugs, 2008, 71, 243; DOI:10.1155/2008/875410

72. Aminabhavi T M, Biradar N S and Patil C S, Inorganica Chimica Acta., 1983, 78, 107-111; DOI:10.1016/S0020-1693(00)86498-1

73. Biradar N S, Aminabhavi T M and Patil C S, Inorganica Chimica Acta., 1983, 78, 47-50; DOI:10.1016/S0020-1693(00)86487-7

74. Tumer M, Celik C, Koksal H and Serin S, Transition Metal Chem., 1999, 24(5), 525532; DOI:10.1023/A:1006982622965

75. Gwaram N S, Mohd Ali H, Khaledi H, Abdulla M A, Hamid A, Hadi A, Kawi Lin T, Ching C L and Ooi C L, Molecules, 2012, 17(5), 5952;

DOI:10.3390/molecules17055952

76. Rudzinski W E and Aminabhavi T M, Inorganica Chimica Acta., 1982, 67, 177-182; DOI:10.1016/S0020-1693(00)85061-6

77. Osowole A A, Wakil S M and Alao O K, World Appl Sci J., 2015, 33(2), 336-342; DOI:10.5829/idosi.wasj.2015.33.02.22206

78. UR-Rehman S, Faiz A and Nawaz R, J Chil Chem Soc., 2013, 58(1), 1624-1627; DOI:10.4067/S0717-97072013000100026.

79. Mohamed G G and Abd El-Wahab Z H, J Thermal Anal Calorimetry, 2003, 73(1), 347-359; DOI:10.1023/A:1025126801265

80. Verma K K, Soni D and verma, Phosphorus, Sulfur Silicon, 2000, 166(1), 231-241; DOI:10.1080/10426500008076544

81. Pant B C, McWhinnie W R and Dance N S, J Organmetal Chem., 1973, 63, 305-310; DOI:10.1016/S0022-328X(73)80043-9

82. Srivastava T N, Singh J D, Indian J. Chem., 1987, 26A, 260.

83. Chauhan S, Garg S and Verma K K, Chem Sci Trans., 2016, 5(2), 431-441; DOI:10.7598/cst2016.1193

84. Kulkarani Y D, Srivastava S, Abdi S H R and Athar M, Synth React Inorg Met Org Chem., 1985, 15(8), 1043-1059; DOI:10.1080/00945718508060634

85. Chauhan S, Garg S and Verma K K, Res J Pharm Biol Chem Sci., 2016, 7(2), 265-274.

86. Aminabhavi T M and Rudzinski W E, Inorganica Chimica Acta., 1983, 76, L131L134; DOI:10.1016/S0020-1693(00)81478-4

87. Aminabhavi T M and Rudzinski W E, Inorganica Chimica Acta., 1983, 78, 51-55; DOI:10.1016/S0020-1693(00)86488-9

88. Cappuccino J C, Sherman N, Microbiology- A Laboratory Manual, Addison Wesley, California, 1999, 263.

89. Pharmacopoeia of India, Volume 1, Controller of Publications, Ministry of Health Department, Government of India, New Delhi, 2007, 37. 


\title{
Synthesis, Spectral and Biological Studies of Some Salicylidene-Trimethoprim Schiff Base Complexes of Aryltellurium(IV)
}

\author{
DEEPAK, K. K. VERMA and SAPANA GARG* \\ Department of Chemistry, Maharshi Dayanand University, Rohtak-124001, India \\ sapanagarg1511@gmail.com
}

Received 14 February 2017 / Accepted 2 March 2017

\begin{abstract}
A novel monobasic tridentate Schiff base salicylidene-trimethoprim, Sal-TMP, synthesized from trimethoprim and salicylaldehyde, form stable complexes with aryltellurium(IV) trichlorides and diaryltellurium(IV) dichlorides of the type Sal-TMP.ArTeCl ${ }_{2}$ and $\mathrm{Sal}_{-} \mathrm{TMP}_{\mathrm{Ar}} \mathrm{AeCl}$ (where $\mathrm{Ar}=p$-methoxyphenyl, $p$-ethoxyphenyl, $p$-hydroxyphenyl and 3-methyl-4-hydroxyphenyl). These have been characterized by elemental analyses, molar conductance, IR and ${ }^{1} \mathrm{H}$ NMR spectroscopy. The spectral studies predict the bonding of ligand through phenolic oxygen of Schiff base after deprotonation, nitrogen of the azomethine group and pyrimidine nitrogen to give hexacoordinated tellurium(IV) complexes. The complexes have also been screened for their antimicrobial activities against various bacteria and fungi organisms.
\end{abstract}

Keywords: Salicylidene-trimethoprim Schiff base, Aryltellurium(IV), Diaryltellurium(IV), Antibacterial, Antifungal activities

\section{Introduction}

Trimethoprim and its derivatives are broad spectrum antimicrobial agents with anti-parasitic activity $^{1-6}$ and were first described by Roth and coworker ${ }^{5}$. Schiff bases, also known as azomethine due to presence of $-\mathrm{C}=\mathrm{N}$ - group, play important role in biological system, such as anticancer $^{7-12}$, antibacterial ${ }^{13-15}$, antiviral ${ }^{16-18}$, antifungal ${ }^{19-21}$ and other biological properties $^{22-27}$ and also have been extensively used as ligands in coordination chemistry because of their excellent donor abilities as chelating agent ${ }^{28-32}$. Medicinal chemists have reported new derivatives of trimethoprim(TMZ) ${ }^{33-35}$ including the Schiff base derived from salicylaldehyde $2-4,36,37$.

Also, aryltellurium(IV) chlorides are known ${ }^{38-54}$ to act as Lewis acids and form complexes with several N-, O- and S- donor bases. In view of this, we herein report some new complexes derived from aryltellurium(IV) trichlorides, $\mathrm{RTeCl}_{3}$ and diaryltellurium(IV) dichlorides, $\mathrm{R}_{2} \mathrm{TeCl}_{2}$ with salicylidene-trimethoprim Schiff base(Sal-TMP). 


\section{Experimental}

All preparations were carried out under dry $\mathrm{N}_{2}$ atmosphere and the solvents used were purified by standard method ${ }^{55,56}$ before use. The purity of compounds was checked by thin layer chromatography using silica gel-G (Merck). Melting points were determined in open capillary tube and are uncorrected.

Carbon, hydrogen and nitrogen analyses were obtained microanalytically from SAIF, Panjab University Chandigarh on a ThermoFinnigan CHNS analyser. Conductivity was measured in DMSO at $25 \pm 2{ }^{\circ} \mathrm{C}$ with dip type conductivity cell on a microprocessor based conductivity bridge type MICROSIL.

IR $\left(4000-400 \mathrm{~cm}^{-1}\right)$ and far IR $\left(400-50 \mathrm{~cm}^{-1}\right)$ spectra were recorded in $\mathrm{KBr} /$ polyethylene pellets on a FT-Infrared spectrophotometer model RZX (Perkin Elmer) at SAIF, Panjab University Chandigarh. Proton magnetic resonance spectra were recorded in DMSO- $\mathrm{d}_{6}$ using TMS as an internal reference on BRUKER AVANCE II 400 NMR spectrometer. The antimicrobial screening was carried out by tube dilution method at Department of Pharmaceutical Sciences, M. D. University, Rohtak, India.

\section{Preparation of aryltellurium(IV) trichlorides and diaryltellurium(IV) dichlorides}

p-Methoxyphenyltellurium(IV) $\quad$ trichloride $^{57,58}, \quad$ bis(p-methoxyphenyl)tellurium(IV) dichloride ${ }^{58,59}, \quad p$-ethoxyphenyltellurium (IV) trichloride ${ }^{60}$, bis( $p$-ethoxyphenyl)tellurium dichloride $^{60} p$-hydroxyphenyltellurium(IV) trichloride ${ }^{61}$, bis( $p$-hydroxyphenyl) tellurium(IV) dichloride $^{61}$, 3-methyl-4-hydroxyphenyltellurium(IV) trichloride $^{62}$ and bis(3-methyl-4hydroxyphenyl)tellurium(IV) dichloride ${ }^{62}$ were prepared by the reactions of $\mathrm{TeCl}_{4}$ with anisole, phenetole, phenol, $o$-cresol respectively, by the methods reported in the literature ${ }^{57-62}$.

\section{Preparation of salicylidene-trimethoprim Schiff base (Sal-TMP)}

Equimolar quantity of saturated methanolic solution of drug and salicylaldehyde were mixed thoroughly. To this mixture $0.1 \%$ methanolic $\mathrm{KOH}$ was added to adjust the $\mathrm{pH}$ of the solution between 7-8 and was refluxed for 2 hours. A clear yellowish-green coloured solution was obtained. After completion of the reaction, the Schiff base ligand was isolated by crystallization after volume reduction by evaporation. The crystalline product was filtered and dried under vacuum and kept in desiccator over $\mathrm{P}_{4} \mathrm{O}_{10}$ until further use. Yield $=$ $80 \%$, M.pt.(decomp.) $=188-190{ }^{\circ} \mathrm{C}$. Analysis (Calculated) $\mathrm{C}_{21} \mathrm{H}_{22} \mathrm{~N}_{4} \mathrm{O}_{4}: \mathrm{C}(63.95), \mathrm{H}(5.62)$ and $\mathrm{N}(14.20)$; Found: $\mathrm{C}(63.50), \mathrm{H}(5.27)$ and $\mathrm{N}(13.99)$.

\section{Preparation of salicylidene-trimethoprim complexes of aryltellurium(IV) trichlorides} and diaryltellurium(IV) dichlorides

Aryltellurium(IV) trichlorides, $\mathrm{ArTeCl}_{3}$ and diaryltellurium(IV) dichlorides $\mathrm{Ar}_{2} \mathrm{TeCl}_{2}$ (Ar= $p$-methoxyphenyl, $p$-ethoxyphenyl, $p$-hydroxyphenyl and 3-methyl-4-hydroxyphenyl), when reacted with sodium salt of salicylidene-trimethoprim in equimolar ratio, yield SalTMP.ArTeCl $\mathrm{Cl}_{2}$ and Sal-TMP.Ar $\mathrm{TeCl}_{2} \mathrm{Type}$ complexes.

Sodium salt of the ligand was prepared by reacting equimolar (1:1) quantity of sodium metal and Schiff base in methanol. The solvent was distilled off to obtain sodium salt of Schiff base. Then a methanolic saturated solution of $2 \mathrm{mmol}$ of aryltellurium(IV) trichloride or diaryltellurium(IV) dichloride was added dropwise to suspension of $2 \mathrm{mmol}$ of sodium salt of Schiff base in about $50 \mathrm{~mL}$ benzene under reflux. The reaction mixture was further refluxed for 3-4 hours, cooled and precipitated sodium chloride was filtered off. The filtrate was then concentrated to about one third of original volume under reduced pressure and cooled in an ice bath to obtain coloured product. This was filtered, washed with benzene + methanol (1:1) and dried in vacuum desiccator over $\mathrm{P}_{4} \mathrm{O}_{10}$. 


\section{Results and Discussion}

$\mathrm{TeCl}_{4}$ when heated with anisole ${ }^{57-59}$, phenetole ${ }^{60}$, phenol ${ }^{61}, o$-cresol ${ }^{62}$ (Ar-H) appears to undergo Friedel-Crafts type condensation reaction whereby $\mathrm{TeCl}_{3}{ }^{+}$unit attacks a position para to the methoxy/ethoxy/hydroxy groups in the aromatic rings, thus resulting in the formation of aryltellurium(IV) trichlorides and diaryltellurium(IV) dichlorides.

$$
\begin{gathered}
\mathrm{Ar}-\mathrm{H}+\mathrm{TeCl}_{4} \longrightarrow \mathrm{ArTeCl}_{3}+\mathrm{HCl} \\
2 \mathrm{Ar}-\mathrm{H}+\mathrm{TeCl}_{4} \longrightarrow \mathrm{Ar}_{2} \mathrm{TeCl}_{2}+2 \mathrm{HCl}
\end{gathered}
$$

Preparation of salicylidene-trimethoprim Schiff base (Sal-TMP) by the reaction of trimethoprim drug and salicylaldehyde can be represented by following equations.

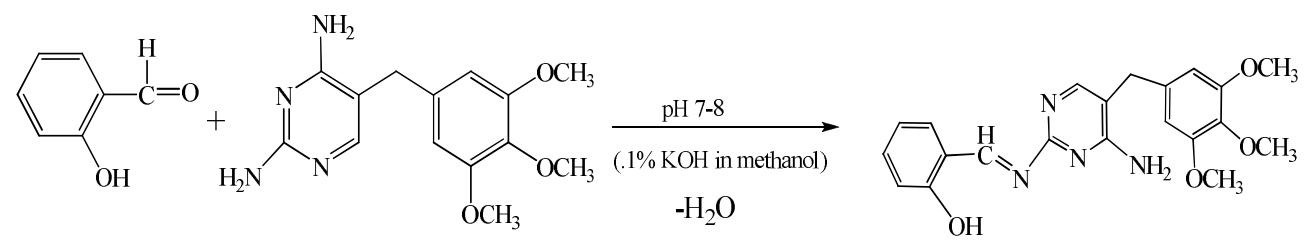

Sodium salt of salicylidene-trimethoprim Schiff base (Sal-TMP) reacts with aryltellurium(IV) trichlorides and diaryltellurium(IV) dichlorides in 1:1 molar ratio to yield the corresponding aryltellurium(IV) complexes.

$$
\begin{aligned}
& \mathrm{Sal}-\mathrm{TMP}+\mathrm{ArTeCl}_{3} \underset{\mathrm{NaCl}}{\stackrel{\mathrm{Na} / \mathrm{CH}_{3} \mathrm{OH}}{\longrightarrow}} \text { (Sal-TMP).ArTeCl } 2 \\
& \mathrm{Sal}-\mathrm{TMP}+\mathrm{Ar}_{2} \mathrm{TeCl}_{2} \underset{-\mathrm{NaCl}}{\stackrel{\mathrm{Na} / \mathrm{CH}_{3} \mathrm{OH}}{\longrightarrow}} \text { (Sal-TMP). } \mathrm{Ar}_{2} \mathrm{TeCl}_{2}
\end{aligned}
$$

All the tellurium(IV) complexes are coloured, crystalline solids, stable at room temperature and non-hygroscopic in nature. The complexes have been analysed for their tellurium, chlorine, carbon, hydrogen and nitrogen contents and the data along with their physical properties and yields are presented in Table 1.

\section{Conductance studies}

Molar conductance $\left(\Lambda_{M}\right)$ data for the complexes in DMSO are complied in Table 1. The $\Lambda_{M}$ value at $c a .10^{-3} \mathrm{M}$ for aryltellurium(IV) complexes in DMSO lie in the range 27.36-91.83 S $\mathrm{cm}^{2} \mathrm{~mol}^{-1}$ which predict the non electrolyte to $1: 1$ electrolyte $^{63,64}$ type behaviour of these complexes in DMSO, probably due to ionization into $\mathrm{ArTeCl}$.Sal- $\mathrm{TMP}^{+} / \mathrm{Ar}_{2} \mathrm{Te}$.Sal-TMP ${ }^{+}$ and $\mathrm{Cl}^{-}$in DMSO. This conductance behavoiur of tellurium(IV) salicylidene-trimethoprim Schiff base complexes is different from those of transition metal complexes ${ }^{65}$, which are reported to be non-electrolytes. The higher $\Lambda_{\mathrm{M}}$ values for some complexes may be due to steric factors and donor behaviour of DMSO to result in probable dissociation into solvated cation and $\mathrm{L}^{-}$along with $\mathrm{Cl}^{-}$in DMSO.

\section{Infrared spectra}

The IR data of Schiff base and its tellurium(IV) complexes are listed in Table 2. The spectra of Sal-TMP Schiff base complexes are quite complex and an attempt has therefore been made to identify the donor sites by comparing the spectra of complexes with parent ligand and $\mathrm{ArTeCl}_{3} / \mathrm{Ar}_{2} \mathrm{TeCl}_{2}$. 
Table 1. Analytical data, molar conductance and physical properties of salicylidene-trimethoprim Schiff base (Sal-TMP) complexes of tellurium(IV)

\begin{tabular}{|c|c|c|c|c|c|c|c|c|c|c|}
\hline \multirow[b]{2}{*}{$\begin{array}{l}\text { Compound } \\
\text { No. }\end{array}$} & \multirow[b]{2}{*}{$\begin{array}{l}\text { Complex } \\
\text { (Ar) }\end{array}$} & \multirow{2}{*}{$\begin{array}{c}\text { Empirical } \\
\text { formula } \\
\text { (Formula Wt.) }\end{array}$} & \multirow[b]{2}{*}{$\begin{array}{l}\text { Colour } \\
\text { (Yield, \%) }\end{array}$} & \multirow{2}{*}{$\begin{array}{l}\text { M. Pt. } \\
{ }^{\circ} \mathrm{C} \\
\text { dec. }\end{array}$} & \multicolumn{5}{|c|}{ Analyses \% Found (Calculated) } & \multirow{2}{*}{$\begin{array}{l}\Lambda_{\mathrm{M}} \text { at } c a \\
10^{-3} \mathrm{M} \mathrm{S} \\
\mathrm{cm}^{2} \mathrm{~mol}^{-1} \\
\text { in DMSO }\end{array}$} \\
\hline & & & & & $\mathrm{C}$ & $\mathrm{H}$ & $\mathrm{N}$ & $\mathrm{Te}$ & $\mathrm{Cl}$ & \\
\hline $\begin{array}{l}\text { Schiff } \\
\text { Base }\end{array}$ & Sal-TMP & $\mathrm{C}_{21} \mathrm{H}_{22} \mathrm{~N}_{4} \mathrm{O}_{4}$ & $\begin{array}{l}\text { Yellowish } \\
\text {-green }(80)\end{array}$ & $188-190$ & $\begin{array}{c}63.50 \\
(63.95)\end{array}$ & $\begin{array}{c}5.27 \\
(5.62)\end{array}$ & $\begin{array}{l}13.99 \\
(14.20)\end{array}$ & - & - & - \\
\hline I & $\begin{array}{l}\text { (Sal-TMP).ArTeCl }{ }_{2} \\
\text { (p-methoxyphenyl) }\end{array}$ & $\begin{array}{c}\mathrm{C}_{28} \mathrm{H}_{28} \mathrm{Cl}_{2} \mathrm{~N}_{4} \mathrm{O}_{5} \mathrm{Te} \\
(699.05)\end{array}$ & $\begin{array}{l}\text { Light cream } \\
(92)\end{array}$ & $230-232$ & $\begin{array}{c}47.88 \\
(48.11)\end{array}$ & $\begin{array}{c}3.99 \\
(4.04)\end{array}$ & $\begin{array}{c}7.75 \\
(8.01)\end{array}$ & $\begin{array}{c}18.07 \\
(18.24)\end{array}$ & $\begin{array}{c}9.89 \\
(10.01)\end{array}$ & 53.19 \\
\hline II & $\begin{array}{c}\text { (Sal-TMP).ArTeCl } \\
\text { (p-ethoxyphenyl) }\end{array}$ & $\begin{array}{c}\mathrm{C}_{29} \mathrm{H}_{30} \mathrm{Cl}_{2} \mathrm{~N}_{4} \mathrm{O}_{5} \mathrm{Te} \\
(713.08)\end{array}$ & $\begin{array}{c}\text { Cream } \\
(85)\end{array}$ & $258-260$ & $\begin{array}{c}48.12 \\
(48.85)\end{array}$ & $\begin{array}{c}3.87 \\
(4.24)\end{array}$ & $\begin{array}{l}7.52 \\
(7.86)\end{array}$ & $\begin{array}{c}17.38 \\
(17.89)\end{array}$ & $\begin{array}{c}9.52 \\
(9.81)\end{array}$ & 52.88 \\
\hline III & $\begin{array}{c}(\mathrm{Sal}-\mathrm{TMP}) \cdot \mathrm{ArTeCl}_{2} \\
(p \text {-hydroxyphenyl })\end{array}$ & $\begin{array}{c}\mathrm{C}_{27} \mathrm{H}_{26} \mathrm{Cl}_{2} \mathrm{~N}_{4} \mathrm{O}_{5} \mathrm{Te} \\
(685.03)\end{array}$ & $\begin{array}{l}\text { Dark cream } \\
\quad(94)\end{array}$ & $208-210$ & $\begin{array}{c}46.78 \\
(47.34)\end{array}$ & $\begin{array}{c}3.50 \\
(3.83)\end{array}$ & $\begin{array}{c}7.88 \\
(8.18)\end{array}$ & $\begin{array}{c}18.28 \\
(18.63)\end{array}$ & $\begin{array}{c}9.98 \\
(10.21)\end{array}$ & 55.73 \\
\hline IV & $\begin{array}{c}\text { (Sal-TMP).ArTeCl } \\
\text { (3-methyl-4- } \\
\text { hydroxyphenyl) }\end{array}$ & $\begin{array}{c}\mathrm{C}_{28} \mathrm{H}_{28} \mathrm{Cl}_{2} \mathrm{~N}_{4} \mathrm{O}_{5} \mathrm{Te} \\
(699.05)\end{array}$ & $\begin{array}{l}\text { Light } \\
\text { cream } \\
(80)\end{array}$ & $198-200$ & $\begin{array}{c}47.58 \\
(48.11)\end{array}$ & $\begin{array}{c}3.84 \\
(4.04)\end{array}$ & $\begin{array}{c}7.80 \\
(8.01)\end{array}$ & $\begin{array}{c}18.15 \\
(18.24)\end{array}$ & $\begin{array}{c}9.85 \\
(10.01)\end{array}$ & 58.68 \\
\hline $\mathrm{V}$ & $\begin{array}{c}(\mathrm{Sal}-\mathrm{TMP}) \cdot \mathrm{Ar}_{2} \mathrm{TeCl} \\
(p \text {-methoxyphenyl })\end{array}$ & $\begin{array}{c}\mathrm{C}_{35} \mathrm{H}_{35} \mathrm{ClN}_{4} \mathrm{O}_{6} \mathrm{Te} \\
(770.73)\end{array}$ & $\begin{array}{c}\text { Pale yellow } \\
\text { (84) }\end{array}$ & $130-132$ & $\begin{array}{c}53.84 \\
(54.54)\end{array}$ & $\begin{array}{c}4.31 \\
(4.58)\end{array}$ & $\begin{array}{c}7.11 \\
(7.27)\end{array}$ & $\begin{array}{c}16.43 \\
(16.56)\end{array}$ & $\begin{array}{c}4.42 \\
(4.60)\end{array}$ & 91.83 \\
\hline VI & $\begin{array}{c}\text { (Sal-TMP).Ar }{ }_{2} \mathrm{TeCl} \\
(p \text {-ethoxyphenyl) }\end{array}$ & $\begin{array}{c}\mathrm{C}_{37} \mathrm{H}_{39} \mathrm{ClN}_{4} \mathrm{O}_{6} \mathrm{Te} \\
(798.78)\end{array}$ & $\begin{array}{l}\text { Light yellow } \\
\text { (86) }\end{array}$ & $150-152$ & $\begin{array}{c}54.84 \\
(55.63)\end{array}$ & $\begin{array}{c}4.53 \\
(4.92)\end{array}$ & $\begin{array}{c}6.84 \\
(7.01)\end{array}$ & $\begin{array}{c}15.50 \\
(15.97)\end{array}$ & $\begin{array}{c}4.30 \\
(4.44)\end{array}$ & 35.90 \\
\hline VII & $\begin{array}{l}\text { (Sal-TMP).Ar } \mathrm{Ar}_{2} \mathrm{TeCl} \\
(p \text {-hydroxyphenyl) }\end{array}$ & $\begin{array}{c}\mathrm{C}_{33} \mathrm{H}_{31} \mathrm{ClN}_{4} \mathrm{O}_{6} \mathrm{Te} \\
(742.68)\end{array}$ & $\begin{array}{l}\text { Red } \\
(89)\end{array}$ & $146-148$ & $\begin{array}{c}52.84 \\
(53.37)\end{array}$ & $\begin{array}{c}4.01 \\
(4.21)\end{array}$ & $\begin{array}{c}7.27 \\
(7.54)\end{array}$ & $\begin{array}{c}16.89 \\
(17.18)\end{array}$ & $\begin{array}{c}4.52 \\
(4.77)\end{array}$ & 36.00 \\
\hline VIII & $\begin{array}{c}\text { (Sal-TMP). } \mathrm{Ar}_{2} \mathrm{TeCl} \\
\text { (3-methyl-4- } \\
\text { hydroxyphenyl) }\end{array}$ & $\begin{array}{c}\mathrm{C}_{35} \mathrm{H}_{35} \mathrm{ClN}_{4} \mathrm{O}_{6} \mathrm{Te} \\
(770.73)\end{array}$ & $\begin{array}{l}\text { Brown } \\
(78)\end{array}$ & $140-142$ & $\begin{array}{l}53.80 \\
(54.54)\end{array}$ & $\begin{array}{c}4.34 \\
(4.58)\end{array}$ & $\begin{array}{c}7.09 \\
(7.27)\end{array}$ & $\begin{array}{c}16.45 \\
(16.56)\end{array}$ & $\begin{array}{c}4.32 \\
(4.60)\end{array}$ & 27.36 \\
\hline
\end{tabular}


Table 2. Important infrared absorption bands $\left(\mathrm{cm}^{-1}\right)$ of Schiff base (Sal-TMP) and complexes

\begin{tabular}{|c|c|c|c|c|c|c|c|}
\hline Compound & $v_{(O-}$ & $v_{(1}$ & $v_{(C}$ & $v_{(C}$ & $v_{(\mathrm{C}-}$ & $v_{(\mathrm{Te}-\mathrm{N})}$ & $v_{(\mathrm{Te}}$ \\
\hline Sal-TMP & $2836 \mathrm{w}$ & $3317 \mathrm{~m} \mathrm{3119} \mathrm{m}$ & $1636 \mathrm{sh}$ & $1633 \mathrm{w} 1593 \mathrm{~s}$ & $1263 \mathrm{~s}$ & - & - \\
\hline $\bar{I}$ & - & $3323 \mathrm{~m} 3184 \mathrm{~m}$ & $1674 \mathrm{mb}$ & $1644 \mathrm{mb} 1587 \mathrm{~s}$ & $1341 \mathrm{~s}$ & $415 \mathrm{~m}$ & $288 \mathrm{w}$ \\
\hline II & - & $3323 \mathrm{~m} 3170 \mathrm{~m}$ & $1647 \mathrm{sh}$ & **1586 s & $1304 \mathrm{~s}$ & $420 \mathrm{~m}$ & $295 w$ \\
\hline III & 3405 m* & $3323 \mathrm{~m} 3150 \mathrm{~m}$ & $1674 \mathrm{mb}$ & $1641 \mathrm{mb} 1586 \mathrm{~s}$ & $1341 \mathrm{~s}$ & $419 \mathrm{~s}$ & $270 \mathrm{w}$ \\
\hline IV & 3398 m* & $3319 \mathrm{~m} 3172 \mathrm{~m}$ & $1652 \mathrm{mb}$ & 1649 & $1333 \mathrm{~s}$ & $450 \mathrm{~s}$ & $277 \mathrm{w}$ \\
\hline V & - & $3325 \mathrm{~m}$ & 164 & $* * 1$ & $1333 \mathrm{~s}$ & $416 \mathrm{~s}$ & $290 \mathrm{w}$ \\
\hline VI & - & $3320 \mathrm{~m} 3158 \mathrm{~m}$ & $1640 \mathrm{sh}$ & $* * 1590 \mathrm{~s}$ & $1331 \mathrm{~s}$ & $410 \mathrm{~m}$ & $273 w$ \\
\hline VII & $3401 \mathrm{w}^{*}$ & $3324 \mathrm{~m} 3168 \mathrm{~m}$ & $1674 \mathrm{mb}$ & $1643 \mathrm{mb} 1584 \mathrm{~s}$ & $1340 \mathrm{~s}$ & $418 \mathrm{~m}$ & $285 \mathrm{w}$ \\
\hline VIII & $3463 \mathrm{w}^{*}$ & $3304 \mathrm{~m} 3106 \mathrm{~m}$ & $1634 \mathrm{sh}$ & $1643 \mathrm{mb} 1584 \mathrm{~s}$ & $1340 \mathrm{~s}$ & $422 \mathrm{~m}$ & $287 \mathrm{w}$ \\
\hline
\end{tabular}

$s=$ sharp, m=medium, mb=medium broad, sh=shoulder, $w=w e a k$, "Due to phenolic $\mathrm{OH}$ of Rte and $\mathrm{R}_{2} \mathrm{Te}$ moieties; ${ }^{* *}$ band not resolved due to overlapping of band $\boldsymbol{v}_{(C=N)}$

Examination of the Schiff base (Sal-TMP) spectrum shows the presence of the hydrogen bonded conjugated- chelate ring system ${ }^{66-68}$ with its centre at $\sim 2832 \mathrm{~cm}^{-1}$. Thus intramolecular $\mathrm{H}$ - bonding is occurring by means of the formation of a quasi six- membered ring involving the $\mathrm{OH}----\mathrm{N}=\mathrm{C}$ bond.<smiles>C1=Cc2ccccc2O[NH+]=1</smiles>

Figure 1. Hydrogen bonding

Thus band disappear on chelation with aryltellurium(IV) chlorides ${ }^{69}$. Hydrogen bond contributes to planarity of the molecule which helps in chelation ${ }^{70}$. Also an intense ligand band at $1263 \mathrm{~cm}^{-1}$ (phenolic -C-O) in free ligand has shifted to higher frequency side in complexes. All these indicate that the hydroxyl group of salicylaldehyde of Schiff base is involved in coordination with tellurium ${ }^{71-73}$.

In addition, the spectra of the Schiff base shown shoulder at $1636 \mathrm{~cm}^{-1}$ with slightly resolved weak band at $1633 \mathrm{~cm}^{-1}$ and sharp band at $1593 \mathrm{~cm}^{-1}$ assigned to $v_{(\mathrm{C}=\mathrm{N})}$ mode for vibration of azomethine group ${ }^{2,4,65,74-76}$ and $v_{(\mathrm{C}=\mathrm{N}) \text { pyrim. For pyrimidine ring }}{ }^{4,77}$. These shift in aryltellurium Schiff base complexes towards higher and lower value ${ }^{74-79}$ reflecting that ligand coordinate through nitrogen atom of azomethine and pyrimidine ring.

The medium intensity band at $3317 \mathrm{~cm}^{-1}$ and $3119 \mathrm{~cm}^{-1}$ due to $v_{(\mathrm{N}-\mathrm{H})}$ asymmetric and symmetric vibrations respectively indicate the non involvement of the nitrogen atom of $\mathrm{NH}_{2}$ group attached to pyrimidine in coordination. The appearance of new weak bands around $270-295 \mathrm{~cm}^{-1}$ due to $v_{(\mathrm{Te}-\mathrm{O})}^{80-83}$ mode and medium to strong band in the range of 410-422 $\mathrm{cm}^{-1}$ due to $v_{(\mathrm{Te}-\mathrm{N})}$ mode ${ }^{84}$ further supports the involvement of phenolic oxygen (after deprotonation), azomethine and pyrimidine nitrogen atoms of Schiff base in the coordination.

Thus, IR data predict the tridentate nature of Sal-TMP involving azomethine nitrogen atom, phenolic oxygen after deprotonation and pyrimidine ring nitrogen giving rise to twosix and four membered chelate rings with the tellurium centre. 


\section{${ }^{1}$ H NMR spectra}

In order to identify the solution structure of Schiff base (Sal-TMP) and its complexes, ${ }^{1} \mathrm{H}$ NMR spectra were recorded in DMSO- $\mathrm{d}_{6}$ and given in Table 3. The proton resonance of the $\mathrm{OH}$ group at $10.92 \delta \mathrm{ppm}^{76,85}$ in Schiff base due to presence of intramolecular hydrogen bonding ${ }^{74}$ disappear on complexation indicating the involvement of phenolic oxygen in the coordination via deprotonation ${ }^{76}$. The azomethine protons which resonate as a singlet at $10.02 \delta \mathrm{ppm}$, the coordination of azomethine nitrogen to tellurium in the complexes are clearly demonstrated by a downfield shift of the peak ${ }^{86}$.

Table 3. ${ }^{1} \mathrm{H}$ NMR spectral data of Schiff base (Sal-TMP) and complexes in DMSO- $\mathrm{d}_{6}$

\begin{tabular}{|c|c|}
\hline Compound & Chemical Shift, $\delta$ ppm \\
\hline Sal-TMP & $\begin{array}{l}2.525(\mathrm{~s}, 2 \mathrm{H}, \text { methylene }), \quad 3.551-3.745\left(\mathrm{~s}, \quad 9 \mathrm{H},-\mathrm{OCH}_{3}\right), \quad 5.672(\mathrm{~s}, 1 \mathrm{H}, \\
\text { pyrimidine }), 6.508\left(\mathrm{~s}, 2 \mathrm{H},-\mathrm{NH}_{2}\right), 7.526-8.169(\mathrm{~m}, 6 \mathrm{H}, \text { aromatic proton), } \\
10.021(\mathrm{~s}, 1 \mathrm{H}, \text { azomethine }), 10.921(\mathrm{~s}, 1 \mathrm{H}, \mathrm{Schiff} \text { base } \mathrm{OH})\end{array}$ \\
\hline I & $\begin{array}{l}2.539(\mathrm{~s}, 2 \mathrm{H}, \text { methylene }), 3.598-3.840\left(\mathrm{~s}, 12 \mathrm{H},-\mathrm{OCH}_{3}\right), 6.574(\mathrm{~s}, 1 \mathrm{H}, \\
\text { pyrimidine }), 6.926\left(\mathrm{~s}, 2 \mathrm{H},-\mathrm{NH}_{2}\right), 7.383-7.522(\mathrm{~m}, 10 \mathrm{H}, \text { aromatic proton }), \\
10.209(\mathrm{~s}, 1 \mathrm{H}, \text { azomethine })\end{array}$ \\
\hline II & $\begin{array}{l}1.335\left(\mathrm{t}, 3 \mathrm{H},-\mathrm{OCH}_{2} \mathrm{CH}_{3}\right), 2.546(\mathrm{~s}, 2 \mathrm{H}, \text { methylene }), 3.963\left(\mathrm{q}, 2 \mathrm{H},-\mathrm{OCH}_{2} \mathrm{CH}_{3}\right) \text {, } \\
3.515-3.875\left(\mathrm{~s}, 9 \mathrm{H},-\mathrm{OCH}_{3}\right), 6.540(\mathrm{~s}, 1 \mathrm{H}, \text { pyrimidine }), 6.950\left(\mathrm{~s}, 2 \mathrm{H},-\mathrm{NH}_{2}\right), \\
6.993-7.809(\mathrm{~m}, 10 \mathrm{H} \text {, aromatic proton), } 10.193(\mathrm{~s}, 1 \mathrm{H} \text {, azomethine })\end{array}$ \\
\hline III & $\begin{array}{l}2.548(\mathrm{~s}, 2 \mathrm{H}, \quad \text { methylene }), \quad 3.601-3.783\left(\mathrm{~s}, 9 \mathrm{H},-\mathrm{OCH}_{3}\right), \quad 6.569(\mathrm{~s}, 1 \mathrm{H}, \\
\text { pyrimidine }), 6.914\left(\mathrm{~s}, 2 \mathrm{H},-\mathrm{NH}_{2}\right), 7.370-7.787(\mathrm{~m}, 10 \mathrm{H}, \text { aromatic proton }), \\
8.155(\mathrm{~s}, 1 \mathrm{H}, \text { phenolic } \mathrm{OH} \text { of } \mathrm{RTe}), 10.201(\mathrm{~s}, 1 \mathrm{H}, \text { azomethine })\end{array}$ \\
\hline IV & $\begin{array}{l}2.536(\mathrm{~s}, 2 \mathrm{H}, \text { methylene }), 2.527\left(\mathrm{~s}, 3 \mathrm{H},-\mathrm{CH}_{3}\right), 3.587-3.770\left(\mathrm{~s}, 9 \mathrm{H},-\mathrm{OCH}_{3}\right) \text {, } \\
\text { 6.605(s, } 1 \mathrm{H}, \text { pyrimidine }), 6.918\left(\mathrm{~s}, 2 \mathrm{H},-\mathrm{NH}_{2}\right), 7.051-7.671(\mathrm{~m}, 9 \mathrm{H} \text {, aromatic } \\
\text { proton }), 8.251(\mathrm{~s}, 1 \mathrm{H} \text {, phenolic } \mathrm{OH} \text { of } \mathrm{RTe}), 10.239(\mathrm{~s}, 1 \mathrm{H} \text {, azomethine })\end{array}$ \\
\hline V & $\begin{array}{l}2.528(\mathrm{~s}, 2 \mathrm{H}, \text { methylene }), 3.673-3.877\left(\mathrm{~s}, 15 \mathrm{H},-\mathrm{OCH}_{3}\right), 6.516(\mathrm{~s}, 1 \mathrm{H} \text {, } \\
\text { pyrimidine }), 6.882\left(\mathrm{~s}, 2 \mathrm{H},-\mathrm{NH}_{2}\right), 6.910-7.804(\mathrm{~m}, 14 \mathrm{H}, \text { aromatic proton }) \\
10.186(\mathrm{~s}, 1 \mathrm{H}, \text { azomethine })\end{array}$ \\
\hline VI & $\begin{array}{l}\left.\text { 1.363(t, } 6 \mathrm{H},-\mathrm{OCH}_{2} \mathbf{C H}_{3}\right), 2.531(\mathrm{~s}, 2 \mathrm{H}, \text { methylene }), 4.019\left(\mathrm{q}, 4 \mathrm{H},-\mathrm{OCH}_{2} \mathrm{CH}_{3}\right) \text {, } \\
3.555-3.765\left(\mathrm{~s}, 9 \mathrm{H},-\mathrm{OCH}_{3}\right), 6.510(\mathrm{~s}, 1 \mathrm{H}, \text { pyrimidine }), 6.924\left(\mathrm{~s}, 2 \mathrm{H},-\mathrm{NH}_{2}\right) \text {, } \\
\text { 7.524-7.805(m, } 14 \mathrm{H} \text {, aromatic proton }), 10.216(\mathrm{~s}, 1 \mathrm{H} \text {, azomethine })\end{array}$ \\
\hline VII & $\begin{array}{l}2.545(\mathrm{~s}, 2 \mathrm{H}, \quad \text { methylene }), 3.568-3.926\left(\mathrm{~s}, 9 \mathrm{H},-\mathrm{OCH}_{3}\right), 6.554(\mathrm{~s}, 1 \mathrm{H} \text {, } \\
\text { pyrimidine }), 6.884\left(\mathrm{~s}, 2 \mathrm{H},-\mathrm{NH}_{2}\right), 7.488-7.678(\mathrm{~m}, 14 \mathrm{H}, \text { aromatic proton), } \\
8.249\left(\mathrm{~s}, 2 \mathrm{H}, \text { phenolic } \mathrm{OH} \text { of } \mathrm{R}_{2} \mathrm{Te}\right), 10.238(\mathrm{~s}, 1 \mathrm{H}, \text { azomethine })\end{array}$ \\
\hline VIII & $\begin{array}{l}2.538(\mathrm{~s}, 2 \mathrm{H}, \text { methylene }), 2.529\left(\mathrm{~s}, 6 \mathrm{H},-\mathrm{CH}_{3}\right), 3.555-3.750\left(\mathrm{~s}, 9 \mathrm{H},-\mathrm{OCH}_{3}\right) \text {, } \\
6.502(\mathrm{~s}, 1 \mathrm{H}, \text { pyrimidine }), 6.926\left(\mathrm{~s}, 2 \mathrm{H},-\mathrm{NH}_{2}\right), 7.126-7.868(\mathrm{~m}, 12 \mathrm{H} \text {, aromatic } \\
\text { proton }), 8.149\left(\mathrm{~s}, 2 \mathrm{H} \text {, phenolic } \mathrm{OH} \text { of } \mathrm{R}_{2} \mathrm{Te}\right), 10.239(\mathrm{~s}, 1 \mathrm{H} \text {, azomethine })\end{array}$ \\
\hline
\end{tabular}
$s=$ singlet,$q=$ quartet,$t=$ triplet, $m=$ multiplet

The characterstic downfield shifting of proton signal in all complexes observed in region $5.67 \delta \mathrm{ppm}$ is due to pyrimidine proton in Schiff base clearly indicate the coordination through pyrimidine nitrogen atom ${ }^{87}$. The signal due to $-\mathrm{NH}$ proton is observed around $6.51 \delta \mathrm{ppm}$ which remain intact with slight variation in complexes is due to the proton bounded to nitrogen experience quadruple effect ${ }^{87}$. Thus salicylidene-trimethoprim act as a tridentate $-\mathrm{N},-\mathrm{N},-\mathrm{O}$ chelating ligand in Sal-TMP.ArTeCl $\mathrm{C}_{2}$ and Sal-TMP. $\mathrm{Ar}_{2} \mathrm{TeCl}$ complexes giving six coordinate tellurium having distorted octahedral geometry in these complexes as predicated from IR studies as well. The proposed structures are as given below (Figure 2). 


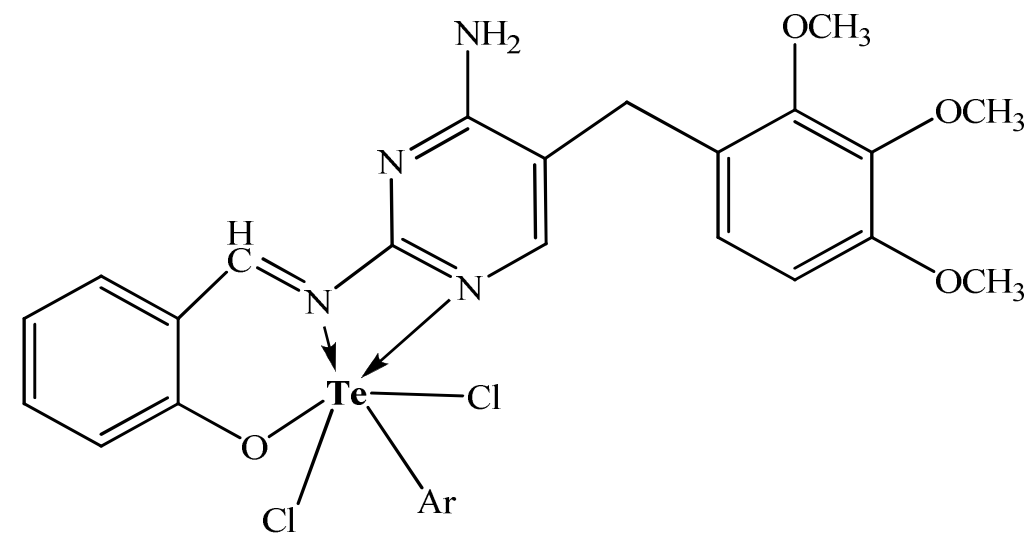<smiles></smiles>

Sal-TMP.Ar ${ }_{2} \mathrm{TeCl}$

$\mathrm{Ar}=p$-methoxyphenyl, $p$-ethoxyphenyl, $p$-hydroxyphenyl and 3-methyl-4-hydroxyphenyl

Figure 2. Proposed structures of complexes

\section{Biological Activity}

The salicylidene-trimethoprim Schiff base (Sal-TMP) and newly synthesized aryltellurium(IV) schiff base complexes were evaluated for their antimicrobial activity in vitro against Gram +ve bacteria (S. aureus ATCC 11632 and B. cereus MTCC 7350), Gram -ve bacteria (E. coli ATCC 35218, P. aeruginosa ATCC 23564, S. typhi ATCC 15499 and $P$. rettgeri DRDE) and fungal strains (A. niger, A. fumigates and A. flavus) by tube dilution method $^{88}$. Dilution of test and standard compounds were prepared double strength nutrient broth- I.P (Antibacterial) and Sabouraud Dextrose Broth -I.P (Antifungal) ${ }^{89}$. The samples were incubated at $37 \pm 1^{\circ} \mathrm{C}$ for $24 \mathrm{~h}$ (bacteria), $25 \pm 1^{\circ} \mathrm{C}$ for 7 days (A. niger), $30 \pm 1^{\circ} \mathrm{C}$ for 15 days (A. flavus), $35 \pm 1{ }^{\circ} \mathrm{C}$ for $72 \mathrm{~h}$ (A. fumigates) respectively and results were recorded in terms of MIC (The lowest concentration of test substances which inhibited values are presented in the Table 4.

The data show that the Sal-TMP complexes of aryltellurium(IV) exhibit more antimicrobial activity towards bacteria as compared to fungii. The antibacterial activity shows following trend. 
Sal-TMP.Ar $\mathrm{ArCl}_{2} \mathrm{Te}$ Sal-TMP.ArTeCl $\mathrm{Cl}_{2} \approx$ Sal-TMP Schiff base

Sal-TMP. $\mathrm{Ar}_{2} \mathrm{TeCl}$ and Sal-TMP.ArTeCl $\mathrm{Cl}_{2}$ complexes have activity towards $S$. typhi and more effectively against $B$. cereus but Schiff base does not show activity against these bacterial strains. Schiff base and its complexes show almost similar activity against fungal strains.

Table 4. Minimum Inhibitory Concentration, MIC, $\mu \mathrm{g} / \mathrm{mL}$; (-) Resistant

\begin{tabular}{|c|c|c|c|c|c|c|c|c|c|}
\hline \multirow[b]{2}{*}{ 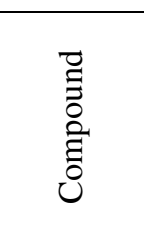 } & \multicolumn{6}{|c|}{ Bacteria strains } & \multicolumn{3}{|c|}{ Fungal strains } \\
\hline & $\begin{array}{c}S . \\
\text { aureus } \\
\text { (ATCC } \\
11632 \text { ) }\end{array}$ & $\begin{array}{l}\text { S. typhi } \\
\text { (ATCC } \\
15499 \text { ) }\end{array}$ & $\begin{array}{c}P . \\
\text { aeruginosa } \\
\text { (ATCC } \\
\text { 23564) }\end{array}$ & $\begin{array}{c}E . \\
a \quad c o l i \\
(\mathrm{ATCC} \\
35218)\end{array}$ & $\begin{array}{c}\text { B. cereus } \\
\text { (MTCC } \\
7350)\end{array}$ & $\begin{array}{c}P . \\
\text { rettgeri } \\
\text { (DRDE } \\
\text { strain) }\end{array}$ & A. niger & $\begin{array}{c}A . \\
\text { fumigates }\end{array}$ & $\begin{array}{l}\text { A. } \\
\text { flavus }\end{array}$ \\
\hline Sal-TMP & 2.5 & - & 1.25 & 5.0 & - & - & 20 & 5.0 & 1.25 \\
\hline I & 2.5 & - & 1.25 & - & 0.625 & 5.0 & - & - & - \\
\hline II & 5.0 & - & 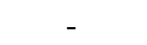 & - & 1.25 & 2.5 & 20 & 5.0 & 1.25 \\
\hline III & - & 20 & 10 & 5.0 & 1.25 & - & 5.0 & - & - \\
\hline IV & 1.25 & 2.5 & 1.25 & 5.0 & - & - & 5.0 & 10 & 5.0 \\
\hline V & - & 20 & 10 & 5.0 & 1.25 & - & 20 & - & - \\
\hline VI & 1.25 & 2.5 & 1.25 & 5.0 & - & - & - & 5.0 & - \\
\hline VII & 1.25 & - & 5.0 & 1.25 & 0.625 & 5.0 & - & 10 & - \\
\hline VIII & 2.5 & - & 1.25 & 5.0 & - & - & 20 & 5.0 & 1.25 \\
\hline
\end{tabular}

\section{Conclusion}

Aryltellurium(IV) and diaryltellurium(IV) dichlorides upon reaction with Schiff base(Sal-TMP) derived from salicylaldehyde and trimethoprim yield new complexes of tellurium(IV). The synthesized complexes were characterized by elemental analyses, conductance measurement, IR and ${ }^{1} \mathrm{H}$ NMR spectral studies. The analytical data suggest that the Sal-TMP Schiff base complexes have 1:1 stoichiometry. The Schiff bases (Sal-TMP) in these complexes functions as a uninegative tridentate ligand through azomethine nitrogen, phenolic oxygen after deprotonation and pyrimidine ring nitrogen atoms. Based on these studies, distorted octahedral geometry with two chelating rings has been assigned to these complexes. The complexes have been observed to possess substantial antimicrobial activity especially against bacteria.

\section{Acknowledgement}

The authors are grateful to M. D. University, Rohtak for providing the necessary facilities. One of the authors (Deepak) is also thankful to UGC New Delhi for providing fellowship. We also thank SAIF, Panjab University Chandigarh for providing the CHN analyses and spectral data.

\section{References}

1. Burchall J J, Corcoran J W and Hahn F E, Eds., Antibiotics, Springer, New York, NY, USA, $1975,3$.

2. Iqbal M S, Khan A H, Loothar B A and Bukhari I H, Med Chem Rev., 2009, 18, 31-42.

3. Blower P J, Аnnu Rep Prog Chem Sect A, 1999, 95, 631-655; DOI:10.1039/A808038K

4. Dhahir S A, Al-Sahib S A, Al Razaq W A and Kadhim N J, Baghdad Sci J., 2010, 7(1), 621.

5. Dr. Karl Thomae Gmb H, (Biberach an der Riss. DE), United States Patent. 4829058.

6. EL-Mossalamy E H, AL-Thabati S A, ALNomaiser F M, Commun Fac Sci Univ Ank Series B, 2005, 51(2), 21-30 
7. Sinha D, Tiwari A K, Singh S, Shukla G, Mishra P, Chandra H and Mishra A K, Eur J Med Chem., 2008, 43(1), 160-165; DOI:10.1016/j.ejmech.2007.03.022

8. Crowe A J, Smith P J and Atassi G, Chem Biol Interact., 1980, 32(1-2), 171-178; DOI:10.1016/0009-2797(80)90075-7

9. Wang M, Wang L F, Li Y Z, Li Q X, Xu Z D and Qu D M, Trans Met Chem., 2001, 26(3), 307-310; DOI:10.1023/A:1007159301849

10. Zishen Wu, Ziqi Gui and Zhenhuan Yen, Synth React Inorg Met Org Chem., 1990, 20(3), 335-344.

11. Desai S B, Desai P B and Desai K R, Hetrocycl Commun., 2001, 7(1), 83-90; DOI:10.1515/HC.2001.7.1.83

12. Dhumwad S D, Gudasi K B and Goudar T R, Indian J Chem., 1994, 33A, 320-324.

13. Przybylski P, Huczynski A, Pyta K, Brzezinski B and Bartl F, Curr Org Chem., 2009, 13(2), 124-148; DOI:10.2174/138527209787193774

14. Pandeya S N, Sriram D, Nath G and De Clercq E, Pharm Acta Hely., 1999, 74(1), 7-11.

15. Karthikeyan M S, Parsad D J, Poojary B, Bhat K S, Holla B S and Kumari N S, Bioorg. Med. Chem., 2006, 14(22), 7482-7489; DOI:10.1016/j.bmc.2006.07.015

16. Holla B S, Akberali P M and Shivananda M K, II Farmaco, 2001, 56(12), 919-927; DOI:10.1016/S0014-827X(01)01124-7

17. Jarrahpour A, Khalili D, De Clercq E, Salmi C and Brunel J M, Molecules, 2007, 12(8), 1720-1730; DOI:10.3390/12081720

18. Da Silva C M, da Silva D L, Modolo L V, Alves R B, de Resende, M A, Martins C V B and de Fatima A J, Adv Res., 2011, 2, 1-8; DOI:10.1016/j.jare.2010.05.004

19. Singh H, Yadav L D S and Mishra S B S, J Inorg Nucl Chem., 1981, 43(7), 17011704; DOI:10.1016/0022-1902(81)80367-3

20. Saravanan G, Pannerselvam P and Prakash C R, J Adv Pharm Techn Res., 2010, 1(3), 320-325; DOI:10.4103/0110-5558.72426

21. Panneerselvam P, Nair R R, Vijayalakshmi G, Subramanian E H and Sridhar S K, Eur. J. Med. Chem. 2005, 40, 225-229; DOI:10.1016/j.ejmech.2004.09.003

22. Singh N K and Singh S B, Indian J Chem., 2001, 40A, 1070-1075.

23. Walsh O M, Meegan M J, Prendergast R M, Nakib T A, Eur J Med Chem., 1996, 31, 989-1000; DOI:10.1016/S0223-5234(97)86178-8

24. Odabasoglu M, Arslan F, Ölmez H and Büyükgüngör O, Dyes and Pigments, 2007, 75(3), 507-515; DOI:10.1016/j.dyepig.2006.06.033

25. Vicini P, Geronikaki A, Incerti M, Busonera B, Poni G, Cabras C A and Colla P L, Bioorg Med Chem., 2003, 11(23), 4785-4789; DOI:10.1016/S09680896(03)00493-0

26. Pandeya S N, Sriram D, Nath G and DeClercq E, Eur J Pharm Sci., 1999, 9, 25-31.

27. Samadhiya S and Halve A, Orient J Chem., 2001, 17, 119-122.

28. Celik Ö, Ulusoy M, Tas E and Ide S, Anal Sciences, 2007, 23, x185.

29. Mahmoud M R, El-Gyar S A, Mousrafa A A and Shaker A, Polyhedron, 1987, 6(5), 1017-1020; DOI:10.1016/S0277-5387(00)80947-X

30. Qing-Yu H, Zheng-Hua M and Ya-Me Z, J Coord Chem., 1990, 21(3), 199-207; DOI:10.1080/00958979009409716

31. Cozzi P G, Chem Soc Rev., 2004, 33, 410-421; DOI:10.1039/B307853C

32. Tarafder M T H, Jin KT, Crouse K A, Ali A M. Yamin, B $M$ and Fun H K, Polyhedron, 2002, 21(25-26), 2547-2554; DOI:10.1016/S0277-5387(02)01188-9

33. Pandeya S N, Sriram D, Clercq E DE., Pannecouque C and Witvrouw M, Indian J Pharm Sci., 1998, 60(4), 207-212. 
34. Fleischman S H and Brooks C L, Proteins: Structure, Function and Genetics, 1990, 7, 52-61.

35. Suling W J, Reynolds R C, Barrow E W, Wilson L N, Piper J R and Barrow W W, J Antimicrobial Chemother., 1998, 42(6), 811-815; DOI:10.1093/jac/42.6.811

36. Gulcan M, Sonmez M and Berber I, Turkey J Chem., 2012, 36(1), 189-200.

37. Raman N, Kulandaisamy A and Jeyasubramanian K, Synth React Inorg Met Org Chem., 2001, 31(7), 1249-1270.

38. Wynne K J and Pearson P S, Inorg Chem., 1971, 10(12), 2735-2739; DOI:10.1021/ic50106a022

39. Wynne K J and Pearson P S, J Chem Soc Commun., 1970, 556-557; DOI:10.1039/C2970000556B

40. Wynne K J, Clark A J and Berg M, J Chem Soc Dalton Trans., 1972, 2370-2374; DOI:10.1039/DT9720002370

41. Clark E R, Collet A J and Naik D G, J Chem Soc Dalton Trans., 1973, 1961-1962; DOI:10.1039/DT9730001961

42. Berg M C, Diss Abstr Int., 1972, 33, 2982.

43. Srivastava T N, Singh M and Singh H B, Indian J Chem., 1982, 21A, 307-309.

44. Srivastava T N, Srivastava R C and Srivastava M, Indian J Chem., 1982, 21A, 539.

45. Srivastava T N, Srivastava R C and Srivastava V K, J Indian Chem Soc., 1983, 60, 891-892.

46. Garad M V, Polyhedron, 1985, 4(8), 1353-1355; DOI:10.1016/S0277-5387(00)86963-6

47. Verma K K and Reena, Synth React Inorg Met-Org Chem., 1999, 29(3), 499-512; DOI:10.1080/00945719909349465

48. Verma K K, Dahiya R and Soni D, Synth React Inorg Met Org Chem., 1999, 29(6), 1033-1052; DOI:10.1080/00945719909349509

49. Verma K K and Dahiya R, Synth React Inorg Met Org Chem., 1999, 29(7), 12991314; DOI:10.1080/00945719909349529

50. Verma K K and Reena, Phosphorus, Sulfur Silicon Related Elements, 1999, 148(1), 227-234; DOI:10.1080/10426509908037013

51. Verma K K and Seema, Int J Chem Sci., 2008, 6, 371-380.

52. Srivastava S, Soni D K and Gupta H S, J Indian Chem Soc., 1996, 73, 255.

53. Narwal J K, Chhabra S, Malik R K, Garg S and Verma K K, Oriental J Chem., 2013, 29, 1339-1349.

54. Chhabra S and Verma K K, J Chem Pharm Res., 2010, 2(4), 569-575.

55. Vogel A I, A Test Book of Organic Chemistry, $3^{\text {rd }}$ Edn., Longman, London, 1975.

56. Weissberger A, Ed., Technique of Organic Chemistry, Vol. 7, $2^{\text {nd }}$ Edn., Interscience Publishers, Inc. N. Y., 1967.

57. Morgan G T and Kellet R E, J Chem Soc., 1926, 1080-1088; DOI:10.1039/JR9262901080

58. Petragnani $\mathrm{N}$ and Stefani $\mathrm{H}$ A, Tellurium in Organic Chemistry, $2^{\text {nd }}$ Edn., Academic Press, London, 2007, 67, 76.

59. Bergman J, Tetrahedron, 1972, 28(12), 3323-3331; DOI:10.1016/S0040-4020(01)93674-9

60. Khandelwal B L, Kumar K and Berry F J, Inorg Chim Acta, 1981, 99(2), 135-137; DOI:10.1016/S0020-1693(00)87958-X

61. Berry F J, Kustan E H, Roshani M and Smith B C, J Organometal Chem., 1975, 99(1), 115-117; DOI:10.1016/S0022-328X(00)86367-6

62. Khandelwal B L, Kumar K and Raina K, Synth React Inorg Met Org Chem., 1981, 11, 65-78.

63. Geary W J, Coord Chem Rev., 1971, 7(1), 81-122; DOI:10.1016/S0010-8545(00)80009-0 
64. Greenwood N N, Straughan B P and Wilson A E, J Chem Soc A, 1968, 2209-2212; DOI: $10.1039 / \mathrm{J} 19680002209$

65. Srivastava K P, Singh A and Singh S K, IOSR J Appl Chem., 2014, 7(4), 16-23; DOI:10.9790/5736-07411623

66. Baker A W and Shulgin A T, J Am Chem Soc., 1959, 81(7), 1523-1529; DOI:10.1021/ja01516a001

67. Freedman H H, J Am Chem Soc., 1961, 83(13), 2900-2905; DOI:10.1021/ja01474a026

68. Flett M St C, Spectrochim Acta, 1957, 10(1), 21-37; DOI:10.1016/0371-1951(57)80160-X

69. Bellamy I J, Infrared Spectra of Complex Molecules, Chapman and Hall Ltd. London, 1975.

70. Matijević-Sosa J, Vinković M and Vikić-Topić D, Croatica Chemica Acta., 2006, 79(3), 489-495.

71. Mishra A P and Soni M, Metal Baesd Drugs, 2008, 71, 243; DOI:10.1155/2008/875410

72. Aminabhavi T M, Biradar N S and Patil C S, Inorganica Chimica Acta., 1983, 78, 107-111; DOI:10.1016/S0020-1693(00)86498-1

73. Biradar N S, Aminabhavi T M and Patil C S, Inorganica Chimica Acta., 1983, 78, 47-50; DOI:10.1016/S0020-1693(00)86487-7

74. Tumer M, Celik C, Koksal H and Serin S, Transition Metal Chem., 1999, 24(5), 525532; DOI:10.1023/A:1006982622965

75. Gwaram N S, Mohd Ali H, Khaledi H, Abdulla M A, Hamid A, Hadi A, Kawi Lin T, Ching C L and Ooi C L, Molecules, 2012, 17(5), 5952;

DOI:10.3390/molecules17055952

76. Rudzinski W E and Aminabhavi T M, Inorganica Chimica Acta., 1982, 67, 177-182; DOI:10.1016/S0020-1693(00)85061-6

77. Osowole A A, Wakil S M and Alao O K, World Appl Sci J., 2015, 33(2), 336-342; DOI:10.5829/idosi.wasj.2015.33.02.22206

78. UR-Rehman S, Faiz A and Nawaz R, J Chil Chem Soc., 2013, 58(1), 1624-1627; DOI:10.4067/S0717-97072013000100026.

79. Mohamed G G and Abd El-Wahab Z H, J Thermal Anal Calorimetry, 2003, 73(1), 347-359; DOI:10.1023/A:1025126801265

80. Verma K K, Soni D and verma, Phosphorus, Sulfur Silicon, 2000, 166(1), 231-241; DOI:10.1080/10426500008076544

81. Pant B C, McWhinnie W R and Dance N S, J Organmetal Chem., 1973, 63, 305-310; DOI:10.1016/S0022-328X(73)80043-9

82. Srivastava T N, Singh J D, Indian J. Chem., 1987, 26A, 260.

83. Chauhan S, Garg S and Verma K K, Chem Sci Trans., 2016, 5(2), 431-441; DOI:10.7598/cst2016.1193

84. Kulkarani Y D, Srivastava S, Abdi S H R and Athar M, Synth React Inorg Met Org Chem., 1985, 15(8), 1043-1059; DOI:10.1080/00945718508060634

85. Chauhan S, Garg S and Verma K K, Res J Pharm Biol Chem Sci., 2016, 7(2), 265-274.

86. Aminabhavi T M and Rudzinski W E, Inorganica Chimica Acta., 1983, 76, L131L134; DOI:10.1016/S0020-1693(00)81478-4

87. Aminabhavi T M and Rudzinski W E, Inorganica Chimica Acta., 1983, 78, 51-55; DOI:10.1016/S0020-1693(00)86488-9

88. Cappuccino J C, Sherman N, Microbiology- A Laboratory Manual, Addison Wesley, California, 1999, 263.

89. Pharmacopoeia of India, Volume 1, Controller of Publications, Ministry of Health Department, Government of India, New Delhi, 2007, 37. 


\title{
Synthesis, Spectral and Biological Studies of Some Salicylidene-Trimethoprim Schiff Base Complexes of Aryltellurium(IV)
}

\author{
DEEPAK, K. K. VERMA and SAPANA GARG* \\ Department of Chemistry, Maharshi Dayanand University, Rohtak-124001, India \\ sapanagarg1511@gmail.com
}

Received 14 February 2017 / Accepted 2 March 2017

\begin{abstract}
A novel monobasic tridentate Schiff base salicylidene-trimethoprim, Sal-TMP, synthesized from trimethoprim and salicylaldehyde, form stable complexes with aryltellurium(IV) trichlorides and diaryltellurium(IV) dichlorides of the type Sal-TMP.ArTeCl ${ }_{2}$ and $\mathrm{Sal}_{-} \mathrm{TMP}_{\mathrm{Ar}} \mathrm{AeCl}$ (where $\mathrm{Ar}=p$-methoxyphenyl, $p$-ethoxyphenyl, $p$-hydroxyphenyl and 3-methyl-4-hydroxyphenyl). These have been characterized by elemental analyses, molar conductance, IR and ${ }^{1} \mathrm{H}$ NMR spectroscopy. The spectral studies predict the bonding of ligand through phenolic oxygen of Schiff base after deprotonation, nitrogen of the azomethine group and pyrimidine nitrogen to give hexacoordinated tellurium(IV) complexes. The complexes have also been screened for their antimicrobial activities against various bacteria and fungi organisms.
\end{abstract}

Keywords: Salicylidene-trimethoprim Schiff base, Aryltellurium(IV), Diaryltellurium(IV), Antibacterial, Antifungal activities

\section{Introduction}

Trimethoprim and its derivatives are broad spectrum antimicrobial agents with anti-parasitic activity $^{1-6}$ and were first described by Roth and coworker ${ }^{5}$. Schiff bases, also known as azomethine due to presence of $-\mathrm{C}=\mathrm{N}$ - group, play important role in biological system, such as anticancer $^{7-12}$, antibacterial ${ }^{13-15}$, antiviral ${ }^{16-18}$, antifungal ${ }^{19-21}$ and other biological properties $^{22-27}$ and also have been extensively used as ligands in coordination chemistry because of their excellent donor abilities as chelating agent ${ }^{28-32}$. Medicinal chemists have reported new derivatives of trimethoprim(TMZ) ${ }^{33-35}$ including the Schiff base derived from salicylaldehyde $2-4,36,37$.

Also, aryltellurium(IV) chlorides are known ${ }^{38-54}$ to act as Lewis acids and form complexes with several N-, O- and S- donor bases. In view of this, we herein report some new complexes derived from aryltellurium(IV) trichlorides, $\mathrm{RTeCl}_{3}$ and diaryltellurium(IV) dichlorides, $\mathrm{R}_{2} \mathrm{TeCl}_{2}$ with salicylidene-trimethoprim Schiff base(Sal-TMP). 


\section{Experimental}

All preparations were carried out under dry $\mathrm{N}_{2}$ atmosphere and the solvents used were purified by standard method ${ }^{55,56}$ before use. The purity of compounds was checked by thin layer chromatography using silica gel-G (Merck). Melting points were determined in open capillary tube and are uncorrected.

Carbon, hydrogen and nitrogen analyses were obtained microanalytically from SAIF, Panjab University Chandigarh on a ThermoFinnigan CHNS analyser. Conductivity was measured in DMSO at $25 \pm 2{ }^{\circ} \mathrm{C}$ with dip type conductivity cell on a microprocessor based conductivity bridge type MICROSIL.

IR $\left(4000-400 \mathrm{~cm}^{-1}\right)$ and far IR $\left(400-50 \mathrm{~cm}^{-1}\right)$ spectra were recorded in $\mathrm{KBr} /$ polyethylene pellets on a FT-Infrared spectrophotometer model RZX (Perkin Elmer) at SAIF, Panjab University Chandigarh. Proton magnetic resonance spectra were recorded in DMSO- $\mathrm{d}_{6}$ using TMS as an internal reference on BRUKER AVANCE II 400 NMR spectrometer. The antimicrobial screening was carried out by tube dilution method at Department of Pharmaceutical Sciences, M. D. University, Rohtak, India.

\section{Preparation of aryltellurium(IV) trichlorides and diaryltellurium(IV) dichlorides}

p-Methoxyphenyltellurium(IV) $\quad$ trichloride $^{57,58}, \quad$ bis(p-methoxyphenyl)tellurium(IV) dichloride ${ }^{58,59}, \quad p$-ethoxyphenyltellurium (IV) trichloride ${ }^{60}$, bis( $p$-ethoxyphenyl)tellurium dichloride $^{60} p$-hydroxyphenyltellurium(IV) trichloride ${ }^{61}$, bis( $p$-hydroxyphenyl) tellurium(IV) dichloride $^{61}$, 3-methyl-4-hydroxyphenyltellurium(IV) trichloride $^{62}$ and bis(3-methyl-4hydroxyphenyl)tellurium(IV) dichloride ${ }^{62}$ were prepared by the reactions of $\mathrm{TeCl}_{4}$ with anisole, phenetole, phenol, $o$-cresol respectively, by the methods reported in the literature ${ }^{57-62}$.

\section{Preparation of salicylidene-trimethoprim Schiff base (Sal-TMP)}

Equimolar quantity of saturated methanolic solution of drug and salicylaldehyde were mixed thoroughly. To this mixture $0.1 \%$ methanolic $\mathrm{KOH}$ was added to adjust the $\mathrm{pH}$ of the solution between 7-8 and was refluxed for 2 hours. A clear yellowish-green coloured solution was obtained. After completion of the reaction, the Schiff base ligand was isolated by crystallization after volume reduction by evaporation. The crystalline product was filtered and dried under vacuum and kept in desiccator over $\mathrm{P}_{4} \mathrm{O}_{10}$ until further use. Yield $=$ $80 \%$, M.pt.(decomp.) $=188-190{ }^{\circ} \mathrm{C}$. Analysis (Calculated) $\mathrm{C}_{21} \mathrm{H}_{22} \mathrm{~N}_{4} \mathrm{O}_{4}: \mathrm{C}(63.95), \mathrm{H}(5.62)$ and $\mathrm{N}(14.20)$; Found: $\mathrm{C}(63.50), \mathrm{H}(5.27)$ and $\mathrm{N}(13.99)$.

\section{Preparation of salicylidene-trimethoprim complexes of aryltellurium(IV) trichlorides} and diaryltellurium(IV) dichlorides

Aryltellurium(IV) trichlorides, $\mathrm{ArTeCl}_{3}$ and diaryltellurium(IV) dichlorides $\mathrm{Ar}_{2} \mathrm{TeCl}_{2}$ (Ar= $p$-methoxyphenyl, $p$-ethoxyphenyl, $p$-hydroxyphenyl and 3-methyl-4-hydroxyphenyl), when reacted with sodium salt of salicylidene-trimethoprim in equimolar ratio, yield SalTMP.ArTeCl $\mathrm{Cl}_{2}$ and Sal-TMP.Ar $\mathrm{TeCl}_{2} \mathrm{Type}$ complexes.

Sodium salt of the ligand was prepared by reacting equimolar (1:1) quantity of sodium metal and Schiff base in methanol. The solvent was distilled off to obtain sodium salt of Schiff base. Then a methanolic saturated solution of $2 \mathrm{mmol}$ of aryltellurium(IV) trichloride or diaryltellurium(IV) dichloride was added dropwise to suspension of $2 \mathrm{mmol}$ of sodium salt of Schiff base in about $50 \mathrm{~mL}$ benzene under reflux. The reaction mixture was further refluxed for 3-4 hours, cooled and precipitated sodium chloride was filtered off. The filtrate was then concentrated to about one third of original volume under reduced pressure and cooled in an ice bath to obtain coloured product. This was filtered, washed with benzene + methanol (1:1) and dried in vacuum desiccator over $\mathrm{P}_{4} \mathrm{O}_{10}$. 


\section{Results and Discussion}

$\mathrm{TeCl}_{4}$ when heated with anisole ${ }^{57-59}$, phenetole ${ }^{60}$, phenol ${ }^{61}, o$-cresol ${ }^{62}$ (Ar-H) appears to undergo Friedel-Crafts type condensation reaction whereby $\mathrm{TeCl}_{3}{ }^{+}$unit attacks a position para to the methoxy/ethoxy/hydroxy groups in the aromatic rings, thus resulting in the formation of aryltellurium(IV) trichlorides and diaryltellurium(IV) dichlorides.

$$
\begin{gathered}
\mathrm{Ar}-\mathrm{H}+\mathrm{TeCl}_{4} \longrightarrow \mathrm{ArTeCl}_{3}+\mathrm{HCl} \\
2 \mathrm{Ar}-\mathrm{H}+\mathrm{TeCl}_{4} \longrightarrow \mathrm{Ar}_{2} \mathrm{TeCl}_{2}+2 \mathrm{HCl}
\end{gathered}
$$

Preparation of salicylidene-trimethoprim Schiff base (Sal-TMP) by the reaction of trimethoprim drug and salicylaldehyde can be represented by following equations.

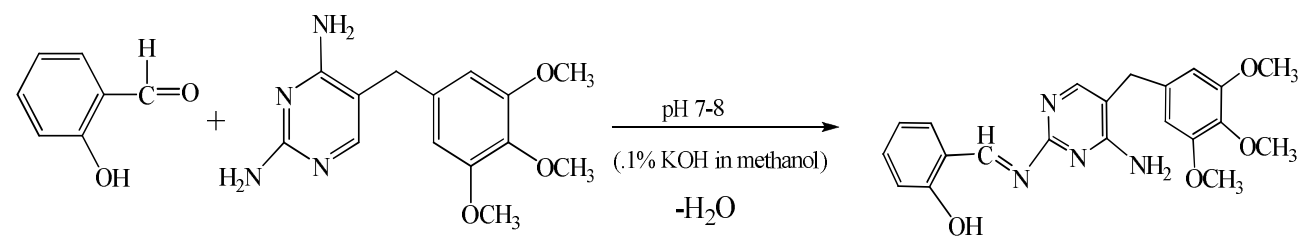

Sodium salt of salicylidene-trimethoprim Schiff base (Sal-TMP) reacts with aryltellurium(IV) trichlorides and diaryltellurium(IV) dichlorides in 1:1 molar ratio to yield the corresponding aryltellurium(IV) complexes.

$$
\begin{aligned}
& \mathrm{Sal}-\mathrm{TMP}+\mathrm{ArTeCl}_{3} \underset{\mathrm{NaCl}}{\stackrel{\mathrm{Na} / \mathrm{CH}_{3} \mathrm{OH}}{\longrightarrow}} \text { (Sal-TMP).ArTeCl } 2 \\
& \mathrm{Sal}-\mathrm{TMP}+\mathrm{Ar}_{2} \mathrm{TeCl}_{2} \underset{-\mathrm{NaCl}}{\stackrel{\mathrm{Na} / \mathrm{CH}_{3} \mathrm{OH}}{\longrightarrow}} \text { (Sal-TMP). } \mathrm{Ar}_{2} \mathrm{TeCl}_{2}
\end{aligned}
$$

All the tellurium(IV) complexes are coloured, crystalline solids, stable at room temperature and non-hygroscopic in nature. The complexes have been analysed for their tellurium, chlorine, carbon, hydrogen and nitrogen contents and the data along with their physical properties and yields are presented in Table 1.

\section{Conductance studies}

Molar conductance $\left(\Lambda_{M}\right)$ data for the complexes in DMSO are complied in Table 1. The $\Lambda_{M}$ value at $c a .10^{-3} \mathrm{M}$ for aryltellurium(IV) complexes in DMSO lie in the range 27.36-91.83 S $\mathrm{cm}^{2} \mathrm{~mol}^{-1}$ which predict the non electrolyte to $1: 1$ electrolyte $^{63,64}$ type behaviour of these complexes in DMSO, probably due to ionization into $\mathrm{ArTeCl}$.Sal- $\mathrm{TMP}^{+} / \mathrm{Ar}_{2} \mathrm{Te}$.Sal-TMP ${ }^{+}$ and $\mathrm{Cl}^{-}$in DMSO. This conductance behavoiur of tellurium(IV) salicylidene-trimethoprim Schiff base complexes is different from those of transition metal complexes ${ }^{65}$, which are reported to be non-electrolytes. The higher $\Lambda_{\mathrm{M}}$ values for some complexes may be due to steric factors and donor behaviour of DMSO to result in probable dissociation into solvated cation and $\mathrm{L}^{-}$along with $\mathrm{Cl}^{-}$in DMSO.

\section{Infrared spectra}

The IR data of Schiff base and its tellurium(IV) complexes are listed in Table 2. The spectra of Sal-TMP Schiff base complexes are quite complex and an attempt has therefore been made to identify the donor sites by comparing the spectra of complexes with parent ligand and $\mathrm{ArTeCl}_{3} / \mathrm{Ar}_{2} \mathrm{TeCl}_{2}$. 
Table 1. Analytical data, molar conductance and physical properties of salicylidene-trimethoprim Schiff base (Sal-TMP) complexes of tellurium(IV)

\begin{tabular}{|c|c|c|c|c|c|c|c|c|c|c|}
\hline \multirow[b]{2}{*}{$\begin{array}{l}\text { Compound } \\
\text { No. }\end{array}$} & \multirow[b]{2}{*}{$\begin{array}{l}\text { Complex } \\
\text { (Ar) }\end{array}$} & \multirow{2}{*}{$\begin{array}{c}\text { Empirical } \\
\text { formula } \\
\text { (Formula Wt.) }\end{array}$} & \multirow[b]{2}{*}{$\begin{array}{l}\text { Colour } \\
\text { (Yield, \%) }\end{array}$} & \multirow{2}{*}{$\begin{array}{l}\text { M. Pt. } \\
{ }^{\circ} \mathrm{C} \\
\text { dec. }\end{array}$} & \multicolumn{5}{|c|}{ Analyses \% Found (Calculated) } & \multirow{2}{*}{$\begin{array}{l}\Lambda_{\mathrm{M}} \text { at } c a \\
10^{-3} \mathrm{M} \mathrm{S} \\
\mathrm{cm}^{2} \mathrm{~mol}^{-1} \\
\text { in DMSO }\end{array}$} \\
\hline & & & & & $\mathrm{C}$ & $\mathrm{H}$ & $\mathrm{N}$ & $\mathrm{Te}$ & $\mathrm{Cl}$ & \\
\hline $\begin{array}{l}\text { Schiff } \\
\text { Base }\end{array}$ & Sal-TMP & $\mathrm{C}_{21} \mathrm{H}_{22} \mathrm{~N}_{4} \mathrm{O}_{4}$ & $\begin{array}{l}\text { Yellowish } \\
\text {-green }(80)\end{array}$ & $188-190$ & $\begin{array}{c}63.50 \\
(63.95)\end{array}$ & $\begin{array}{c}5.27 \\
(5.62)\end{array}$ & $\begin{array}{l}13.99 \\
(14.20)\end{array}$ & - & - & - \\
\hline I & $\begin{array}{l}\text { (Sal-TMP).ArTeCl }{ }_{2} \\
\text { (p-methoxyphenyl) }\end{array}$ & $\begin{array}{c}\mathrm{C}_{28} \mathrm{H}_{28} \mathrm{Cl}_{2} \mathrm{~N}_{4} \mathrm{O}_{5} \mathrm{Te} \\
(699.05)\end{array}$ & $\begin{array}{l}\text { Light cream } \\
(92)\end{array}$ & $230-232$ & $\begin{array}{c}47.88 \\
(48.11)\end{array}$ & $\begin{array}{c}3.99 \\
(4.04)\end{array}$ & $\begin{array}{c}7.75 \\
(8.01)\end{array}$ & $\begin{array}{c}18.07 \\
(18.24)\end{array}$ & $\begin{array}{c}9.89 \\
(10.01)\end{array}$ & 53.19 \\
\hline II & $\begin{array}{c}\text { (Sal-TMP).ArTeCl } \\
\text { (p-ethoxyphenyl) }\end{array}$ & $\begin{array}{c}\mathrm{C}_{29} \mathrm{H}_{30} \mathrm{Cl}_{2} \mathrm{~N}_{4} \mathrm{O}_{5} \mathrm{Te} \\
(713.08)\end{array}$ & $\begin{array}{c}\text { Cream } \\
(85)\end{array}$ & $258-260$ & $\begin{array}{c}48.12 \\
(48.85)\end{array}$ & $\begin{array}{c}3.87 \\
(4.24)\end{array}$ & $\begin{array}{l}7.52 \\
(7.86)\end{array}$ & $\begin{array}{c}17.38 \\
(17.89)\end{array}$ & $\begin{array}{c}9.52 \\
(9.81)\end{array}$ & 52.88 \\
\hline III & $\begin{array}{c}(\mathrm{Sal}-\mathrm{TMP}) \cdot \mathrm{ArTeCl}_{2} \\
(p \text {-hydroxyphenyl })\end{array}$ & $\begin{array}{c}\mathrm{C}_{27} \mathrm{H}_{26} \mathrm{Cl}_{2} \mathrm{~N}_{4} \mathrm{O}_{5} \mathrm{Te} \\
(685.03)\end{array}$ & $\begin{array}{l}\text { Dark cream } \\
\quad(94)\end{array}$ & $208-210$ & $\begin{array}{c}46.78 \\
(47.34)\end{array}$ & $\begin{array}{c}3.50 \\
(3.83)\end{array}$ & $\begin{array}{c}7.88 \\
(8.18)\end{array}$ & $\begin{array}{c}18.28 \\
(18.63)\end{array}$ & $\begin{array}{c}9.98 \\
(10.21)\end{array}$ & 55.73 \\
\hline IV & $\begin{array}{c}\text { (Sal-TMP).ArTeCl } \\
\text { (3-methyl-4- } \\
\text { hydroxyphenyl) }\end{array}$ & $\begin{array}{c}\mathrm{C}_{28} \mathrm{H}_{28} \mathrm{Cl}_{2} \mathrm{~N}_{4} \mathrm{O}_{5} \mathrm{Te} \\
(699.05)\end{array}$ & $\begin{array}{l}\text { Light } \\
\text { cream } \\
(80)\end{array}$ & $198-200$ & $\begin{array}{c}47.58 \\
(48.11)\end{array}$ & $\begin{array}{c}3.84 \\
(4.04)\end{array}$ & $\begin{array}{c}7.80 \\
(8.01)\end{array}$ & $\begin{array}{c}18.15 \\
(18.24)\end{array}$ & $\begin{array}{c}9.85 \\
(10.01)\end{array}$ & 58.68 \\
\hline $\mathrm{V}$ & $\begin{array}{c}(\mathrm{Sal}-\mathrm{TMP}) \cdot \mathrm{Ar}_{2} \mathrm{TeCl} \\
(p \text {-methoxyphenyl })\end{array}$ & $\begin{array}{c}\mathrm{C}_{35} \mathrm{H}_{35} \mathrm{ClN}_{4} \mathrm{O}_{6} \mathrm{Te} \\
(770.73)\end{array}$ & $\begin{array}{c}\text { Pale yellow } \\
\text { (84) }\end{array}$ & $130-132$ & $\begin{array}{c}53.84 \\
(54.54)\end{array}$ & $\begin{array}{c}4.31 \\
(4.58)\end{array}$ & $\begin{array}{c}7.11 \\
(7.27)\end{array}$ & $\begin{array}{c}16.43 \\
(16.56)\end{array}$ & $\begin{array}{c}4.42 \\
(4.60)\end{array}$ & 91.83 \\
\hline VI & $\begin{array}{c}\text { (Sal-TMP).Ar }{ }_{2} \mathrm{TeCl} \\
(p \text {-ethoxyphenyl) }\end{array}$ & $\begin{array}{c}\mathrm{C}_{37} \mathrm{H}_{39} \mathrm{ClN}_{4} \mathrm{O}_{6} \mathrm{Te} \\
(798.78)\end{array}$ & $\begin{array}{l}\text { Light yellow } \\
\text { (86) }\end{array}$ & $150-152$ & $\begin{array}{c}54.84 \\
(55.63)\end{array}$ & $\begin{array}{c}4.53 \\
(4.92)\end{array}$ & $\begin{array}{c}6.84 \\
(7.01)\end{array}$ & $\begin{array}{c}15.50 \\
(15.97)\end{array}$ & $\begin{array}{c}4.30 \\
(4.44)\end{array}$ & 35.90 \\
\hline VII & $\begin{array}{l}\text { (Sal-TMP).Ar } \mathrm{Ar}_{2} \mathrm{TeCl} \\
(p \text {-hydroxyphenyl) }\end{array}$ & $\begin{array}{c}\mathrm{C}_{33} \mathrm{H}_{31} \mathrm{ClN}_{4} \mathrm{O}_{6} \mathrm{Te} \\
(742.68)\end{array}$ & $\begin{array}{l}\text { Red } \\
(89)\end{array}$ & $146-148$ & $\begin{array}{c}52.84 \\
(53.37)\end{array}$ & $\begin{array}{c}4.01 \\
(4.21)\end{array}$ & $\begin{array}{c}7.27 \\
(7.54)\end{array}$ & $\begin{array}{c}16.89 \\
(17.18)\end{array}$ & $\begin{array}{c}4.52 \\
(4.77)\end{array}$ & 36.00 \\
\hline VIII & $\begin{array}{c}\text { (Sal-TMP). } \mathrm{Ar}_{2} \mathrm{TeCl} \\
\text { (3-methyl-4- } \\
\text { hydroxyphenyl) }\end{array}$ & $\begin{array}{c}\mathrm{C}_{35} \mathrm{H}_{35} \mathrm{ClN}_{4} \mathrm{O}_{6} \mathrm{Te} \\
(770.73)\end{array}$ & $\begin{array}{l}\text { Brown } \\
(78)\end{array}$ & $140-142$ & $\begin{array}{l}53.80 \\
(54.54)\end{array}$ & $\begin{array}{c}4.34 \\
(4.58)\end{array}$ & $\begin{array}{c}7.09 \\
(7.27)\end{array}$ & $\begin{array}{c}16.45 \\
(16.56)\end{array}$ & $\begin{array}{c}4.32 \\
(4.60)\end{array}$ & 27.36 \\
\hline
\end{tabular}


Table 2. Important infrared absorption bands $\left(\mathrm{cm}^{-1}\right)$ of Schiff base (Sal-TMP) and complexes

\begin{tabular}{|c|c|c|c|c|c|c|c|}
\hline Compound & $v_{(O-}$ & $v_{(1}$ & $v_{(C}$ & $v_{(C}$ & $v_{(\mathrm{C}-}$ & $v_{(\mathrm{Te}-\mathrm{N})}$ & $v_{(\mathrm{Te}}$ \\
\hline Sal-TMP & $2836 \mathrm{w}$ & $3317 \mathrm{~m} \mathrm{3119} \mathrm{m}$ & $1636 \mathrm{sh}$ & $1633 \mathrm{w} 1593 \mathrm{~s}$ & $1263 \mathrm{~s}$ & - & - \\
\hline $\bar{I}$ & - & $3323 \mathrm{~m} 3184 \mathrm{~m}$ & $1674 \mathrm{mb}$ & $1644 \mathrm{mb} 1587 \mathrm{~s}$ & $1341 \mathrm{~s}$ & $415 \mathrm{~m}$ & $288 \mathrm{w}$ \\
\hline II & - & $3323 \mathrm{~m} 3170 \mathrm{~m}$ & $1647 \mathrm{sh}$ & **1586 s & $1304 \mathrm{~s}$ & $420 \mathrm{~m}$ & $295 w$ \\
\hline III & 3405 m* & $3323 \mathrm{~m} 3150 \mathrm{~m}$ & $1674 \mathrm{mb}$ & $1641 \mathrm{mb} 1586 \mathrm{~s}$ & $1341 \mathrm{~s}$ & $419 \mathrm{~s}$ & $270 \mathrm{w}$ \\
\hline IV & 3398 m* & $3319 \mathrm{~m} 3172 \mathrm{~m}$ & $1652 \mathrm{mb}$ & 1649 & $1333 \mathrm{~s}$ & $450 \mathrm{~s}$ & $277 \mathrm{w}$ \\
\hline V & - & $3325 \mathrm{~m}$ & 164 & $* * 1$ & $1333 \mathrm{~s}$ & $416 \mathrm{~s}$ & $290 \mathrm{w}$ \\
\hline VI & - & $3320 \mathrm{~m} 3158 \mathrm{~m}$ & $1640 \mathrm{sh}$ & $* * 1590 \mathrm{~s}$ & $1331 \mathrm{~s}$ & $410 \mathrm{~m}$ & $273 w$ \\
\hline VII & $3401 \mathrm{w}^{*}$ & $3324 \mathrm{~m} 3168 \mathrm{~m}$ & $1674 \mathrm{mb}$ & $1643 \mathrm{mb} 1584 \mathrm{~s}$ & $1340 \mathrm{~s}$ & $418 \mathrm{~m}$ & $285 \mathrm{w}$ \\
\hline VIII & $3463 \mathrm{w}^{*}$ & $3304 \mathrm{~m} 3106 \mathrm{~m}$ & $1634 \mathrm{sh}$ & $1643 \mathrm{mb} 1584 \mathrm{~s}$ & $1340 \mathrm{~s}$ & $422 \mathrm{~m}$ & $287 \mathrm{w}$ \\
\hline
\end{tabular}

$s=$ sharp, m=medium, mb=medium broad, sh=shoulder, $w=w e a k$, "Due to phenolic $\mathrm{OH}$ of Rte and $\mathrm{R}_{2} \mathrm{Te}$ moieties; ${ }^{* *}$ band not resolved due to overlapping of band $\boldsymbol{v}_{(C=N)}$

Examination of the Schiff base (Sal-TMP) spectrum shows the presence of the hydrogen bonded conjugated- chelate ring system ${ }^{66-68}$ with its centre at $\sim 2832 \mathrm{~cm}^{-1}$. Thus intramolecular $\mathrm{H}$ - bonding is occurring by means of the formation of a quasi six- membered ring involving the $\mathrm{OH}----\mathrm{N}=\mathrm{C}$ bond.<smiles>C1=Cc2ccccc2O[NH+]=1</smiles>

Figure 1. Hydrogen bonding

Thus band disappear on chelation with aryltellurium(IV) chlorides ${ }^{69}$. Hydrogen bond contributes to planarity of the molecule which helps in chelation ${ }^{70}$. Also an intense ligand band at $1263 \mathrm{~cm}^{-1}$ (phenolic -C-O) in free ligand has shifted to higher frequency side in complexes. All these indicate that the hydroxyl group of salicylaldehyde of Schiff base is involved in coordination with tellurium ${ }^{71-73}$.

In addition, the spectra of the Schiff base shown shoulder at $1636 \mathrm{~cm}^{-1}$ with slightly resolved weak band at $1633 \mathrm{~cm}^{-1}$ and sharp band at $1593 \mathrm{~cm}^{-1}$ assigned to $v_{(\mathrm{C}=\mathrm{N})}$ mode for vibration of azomethine group ${ }^{2,4,65,74-76}$ and $v_{(\mathrm{C}=\mathrm{N}) \text { pyrim. For pyrimidine ring }}{ }^{4,77}$. These shift in aryltellurium Schiff base complexes towards higher and lower value ${ }^{74-79}$ reflecting that ligand coordinate through nitrogen atom of azomethine and pyrimidine ring.

The medium intensity band at $3317 \mathrm{~cm}^{-1}$ and $3119 \mathrm{~cm}^{-1}$ due to $v_{(\mathrm{N}-\mathrm{H})}$ asymmetric and symmetric vibrations respectively indicate the non involvement of the nitrogen atom of $\mathrm{NH}_{2}$ group attached to pyrimidine in coordination. The appearance of new weak bands around $270-295 \mathrm{~cm}^{-1}$ due to $v_{(\mathrm{Te}-\mathrm{O})}^{80-83}$ mode and medium to strong band in the range of 410-422 $\mathrm{cm}^{-1}$ due to $v_{(\mathrm{Te}-\mathrm{N})}$ mode ${ }^{84}$ further supports the involvement of phenolic oxygen (after deprotonation), azomethine and pyrimidine nitrogen atoms of Schiff base in the coordination.

Thus, IR data predict the tridentate nature of Sal-TMP involving azomethine nitrogen atom, phenolic oxygen after deprotonation and pyrimidine ring nitrogen giving rise to twosix and four membered chelate rings with the tellurium centre. 


\section{${ }^{1}$ H NMR spectra}

In order to identify the solution structure of Schiff base (Sal-TMP) and its complexes, ${ }^{1} \mathrm{H}$ NMR spectra were recorded in DMSO- $\mathrm{d}_{6}$ and given in Table 3. The proton resonance of the $\mathrm{OH}$ group at $10.92 \delta \mathrm{ppm}^{76,85}$ in Schiff base due to presence of intramolecular hydrogen bonding ${ }^{74}$ disappear on complexation indicating the involvement of phenolic oxygen in the coordination via deprotonation ${ }^{76}$. The azomethine protons which resonate as a singlet at $10.02 \delta \mathrm{ppm}$, the coordination of azomethine nitrogen to tellurium in the complexes are clearly demonstrated by a downfield shift of the peak ${ }^{86}$.

Table 3. ${ }^{1} \mathrm{H}$ NMR spectral data of Schiff base (Sal-TMP) and complexes in DMSO- $\mathrm{d}_{6}$

\begin{tabular}{|c|c|}
\hline Compound & Chemical Shift, $\delta$ ppm \\
\hline Sal-TMP & $\begin{array}{l}2.525(\mathrm{~s}, 2 \mathrm{H}, \text { methylene }), \quad 3.551-3.745\left(\mathrm{~s}, \quad 9 \mathrm{H},-\mathrm{OCH}_{3}\right), \quad 5.672(\mathrm{~s}, 1 \mathrm{H}, \\
\text { pyrimidine }), 6.508\left(\mathrm{~s}, 2 \mathrm{H},-\mathrm{NH}_{2}\right), 7.526-8.169(\mathrm{~m}, 6 \mathrm{H}, \text { aromatic proton), } \\
10.021(\mathrm{~s}, 1 \mathrm{H}, \text { azomethine }), 10.921(\mathrm{~s}, 1 \mathrm{H}, \mathrm{Schiff} \text { base } \mathrm{OH})\end{array}$ \\
\hline I & $\begin{array}{l}2.539(\mathrm{~s}, 2 \mathrm{H}, \text { methylene }), 3.598-3.840\left(\mathrm{~s}, 12 \mathrm{H},-\mathrm{OCH}_{3}\right), 6.574(\mathrm{~s}, 1 \mathrm{H}, \\
\text { pyrimidine }), 6.926\left(\mathrm{~s}, 2 \mathrm{H},-\mathrm{NH}_{2}\right), 7.383-7.522(\mathrm{~m}, 10 \mathrm{H}, \text { aromatic proton }), \\
10.209(\mathrm{~s}, 1 \mathrm{H}, \text { azomethine })\end{array}$ \\
\hline II & $\begin{array}{l}1.335\left(\mathrm{t}, 3 \mathrm{H},-\mathrm{OCH}_{2} \mathrm{CH}_{3}\right), 2.546(\mathrm{~s}, 2 \mathrm{H}, \text { methylene }), 3.963\left(\mathrm{q}, 2 \mathrm{H},-\mathrm{OCH}_{2} \mathrm{CH}_{3}\right) \text {, } \\
3.515-3.875\left(\mathrm{~s}, 9 \mathrm{H},-\mathrm{OCH}_{3}\right), 6.540(\mathrm{~s}, 1 \mathrm{H}, \text { pyrimidine }), 6.950\left(\mathrm{~s}, 2 \mathrm{H},-\mathrm{NH}_{2}\right), \\
6.993-7.809(\mathrm{~m}, 10 \mathrm{H} \text {, aromatic proton), } 10.193(\mathrm{~s}, 1 \mathrm{H} \text {, azomethine })\end{array}$ \\
\hline III & $\begin{array}{l}2.548(\mathrm{~s}, 2 \mathrm{H}, \quad \text { methylene }), \quad 3.601-3.783\left(\mathrm{~s}, 9 \mathrm{H},-\mathrm{OCH}_{3}\right), \quad 6.569(\mathrm{~s}, 1 \mathrm{H}, \\
\text { pyrimidine }), 6.914\left(\mathrm{~s}, 2 \mathrm{H},-\mathrm{NH}_{2}\right), 7.370-7.787(\mathrm{~m}, 10 \mathrm{H}, \text { aromatic proton }), \\
8.155(\mathrm{~s}, 1 \mathrm{H}, \text { phenolic } \mathrm{OH} \text { of } \mathrm{RTe}), 10.201(\mathrm{~s}, 1 \mathrm{H}, \text { azomethine })\end{array}$ \\
\hline IV & $\begin{array}{l}2.536(\mathrm{~s}, 2 \mathrm{H}, \text { methylene }), 2.527\left(\mathrm{~s}, 3 \mathrm{H},-\mathrm{CH}_{3}\right), 3.587-3.770\left(\mathrm{~s}, 9 \mathrm{H},-\mathrm{OCH}_{3}\right) \text {, } \\
\text { 6.605(s, } 1 \mathrm{H}, \text { pyrimidine }), 6.918\left(\mathrm{~s}, 2 \mathrm{H},-\mathrm{NH}_{2}\right), 7.051-7.671(\mathrm{~m}, 9 \mathrm{H} \text {, aromatic } \\
\text { proton }), 8.251(\mathrm{~s}, 1 \mathrm{H} \text {, phenolic } \mathrm{OH} \text { of } \mathrm{RTe}), 10.239(\mathrm{~s}, 1 \mathrm{H} \text {, azomethine })\end{array}$ \\
\hline V & $\begin{array}{l}2.528(\mathrm{~s}, 2 \mathrm{H}, \text { methylene }), 3.673-3.877\left(\mathrm{~s}, 15 \mathrm{H},-\mathrm{OCH}_{3}\right), 6.516(\mathrm{~s}, 1 \mathrm{H} \text {, } \\
\text { pyrimidine }), 6.882\left(\mathrm{~s}, 2 \mathrm{H},-\mathrm{NH}_{2}\right), 6.910-7.804(\mathrm{~m}, 14 \mathrm{H}, \text { aromatic proton }) \\
10.186(\mathrm{~s}, 1 \mathrm{H}, \text { azomethine })\end{array}$ \\
\hline VI & $\begin{array}{l}\left.\text { 1.363(t, } 6 \mathrm{H},-\mathrm{OCH}_{2} \mathbf{C H}_{3}\right), 2.531(\mathrm{~s}, 2 \mathrm{H}, \text { methylene }), 4.019\left(\mathrm{q}, 4 \mathrm{H},-\mathrm{OCH}_{2} \mathrm{CH}_{3}\right) \text {, } \\
3.555-3.765\left(\mathrm{~s}, 9 \mathrm{H},-\mathrm{OCH}_{3}\right), 6.510(\mathrm{~s}, 1 \mathrm{H}, \text { pyrimidine }), 6.924\left(\mathrm{~s}, 2 \mathrm{H},-\mathrm{NH}_{2}\right) \text {, } \\
\text { 7.524-7.805(m, } 14 \mathrm{H} \text {, aromatic proton }), 10.216(\mathrm{~s}, 1 \mathrm{H} \text {, azomethine })\end{array}$ \\
\hline VII & $\begin{array}{l}2.545(\mathrm{~s}, 2 \mathrm{H}, \quad \text { methylene }), 3.568-3.926\left(\mathrm{~s}, 9 \mathrm{H},-\mathrm{OCH}_{3}\right), 6.554(\mathrm{~s}, 1 \mathrm{H} \text {, } \\
\text { pyrimidine }), 6.884\left(\mathrm{~s}, 2 \mathrm{H},-\mathrm{NH}_{2}\right), 7.488-7.678(\mathrm{~m}, 14 \mathrm{H}, \text { aromatic proton), } \\
8.249\left(\mathrm{~s}, 2 \mathrm{H}, \text { phenolic } \mathrm{OH} \text { of } \mathrm{R}_{2} \mathrm{Te}\right), 10.238(\mathrm{~s}, 1 \mathrm{H}, \text { azomethine })\end{array}$ \\
\hline VIII & $\begin{array}{l}2.538(\mathrm{~s}, 2 \mathrm{H}, \text { methylene }), 2.529\left(\mathrm{~s}, 6 \mathrm{H},-\mathrm{CH}_{3}\right), 3.555-3.750\left(\mathrm{~s}, 9 \mathrm{H},-\mathrm{OCH}_{3}\right) \text {, } \\
6.502(\mathrm{~s}, 1 \mathrm{H}, \text { pyrimidine }), 6.926\left(\mathrm{~s}, 2 \mathrm{H},-\mathrm{NH}_{2}\right), 7.126-7.868(\mathrm{~m}, 12 \mathrm{H} \text {, aromatic } \\
\text { proton }), 8.149\left(\mathrm{~s}, 2 \mathrm{H} \text {, phenolic } \mathrm{OH} \text { of } \mathrm{R}_{2} \mathrm{Te}\right), 10.239(\mathrm{~s}, 1 \mathrm{H} \text {, azomethine })\end{array}$ \\
\hline
\end{tabular}
$s=$ singlet,$q=$ quartet,$t=$ triplet, $m=$ multiplet

The characterstic downfield shifting of proton signal in all complexes observed in region $5.67 \delta \mathrm{ppm}$ is due to pyrimidine proton in Schiff base clearly indicate the coordination through pyrimidine nitrogen atom ${ }^{87}$. The signal due to $-\mathrm{NH}$ proton is observed around $6.51 \delta \mathrm{ppm}$ which remain intact with slight variation in complexes is due to the proton bounded to nitrogen experience quadruple effect ${ }^{87}$. Thus salicylidene-trimethoprim act as a tridentate $-\mathrm{N},-\mathrm{N},-\mathrm{O}$ chelating ligand in Sal-TMP.ArTeCl $\mathrm{C}_{2}$ and Sal-TMP. $\mathrm{Ar}_{2} \mathrm{TeCl}$ complexes giving six coordinate tellurium having distorted octahedral geometry in these complexes as predicated from IR studies as well. The proposed structures are as given below (Figure 2). 


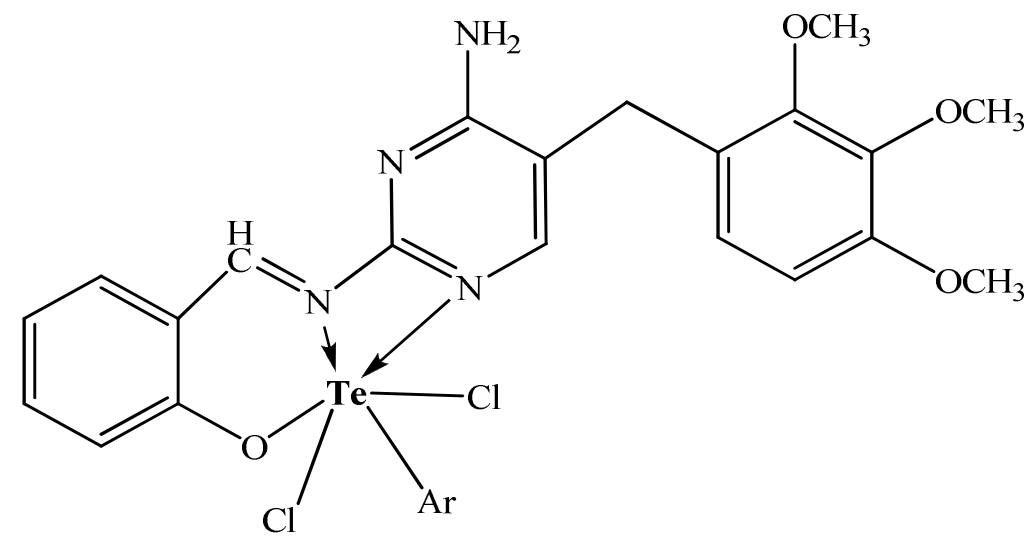<smiles></smiles>

Sal-TMP.Ar ${ }_{2} \mathrm{TeCl}$

$\mathrm{Ar}=p$-methoxyphenyl, $p$-ethoxyphenyl, $p$-hydroxyphenyl and 3-methyl-4-hydroxyphenyl

Figure 2. Proposed structures of complexes

\section{Biological Activity}

The salicylidene-trimethoprim Schiff base (Sal-TMP) and newly synthesized aryltellurium(IV) schiff base complexes were evaluated for their antimicrobial activity in vitro against Gram +ve bacteria (S. aureus ATCC 11632 and B. cereus MTCC 7350), Gram -ve bacteria (E. coli ATCC 35218, P. aeruginosa ATCC 23564, S. typhi ATCC 15499 and $P$. rettgeri DRDE) and fungal strains (A. niger, A. fumigates and A. flavus) by tube dilution method $^{88}$. Dilution of test and standard compounds were prepared double strength nutrient broth- I.P (Antibacterial) and Sabouraud Dextrose Broth -I.P (Antifungal) ${ }^{89}$. The samples were incubated at $37 \pm 1^{\circ} \mathrm{C}$ for $24 \mathrm{~h}$ (bacteria), $25 \pm 1^{\circ} \mathrm{C}$ for 7 days (A. niger), $30 \pm 1^{\circ} \mathrm{C}$ for 15 days (A. flavus), $35 \pm 1{ }^{\circ} \mathrm{C}$ for $72 \mathrm{~h}$ (A. fumigates) respectively and results were recorded in terms of MIC (The lowest concentration of test substances which inhibited values are presented in the Table 4.

The data show that the Sal-TMP complexes of aryltellurium(IV) exhibit more antimicrobial activity towards bacteria as compared to fungii. The antibacterial activity shows following trend. 
Sal-TMP.Ar $\mathrm{ArCl}_{2} \mathrm{Te}$ Sal-TMP.ArTeCl $\mathrm{Cl}_{2} \approx$ Sal-TMP Schiff base

Sal-TMP. $\mathrm{Ar}_{2} \mathrm{TeCl}$ and Sal-TMP.ArTeCl $\mathrm{Cl}_{2}$ complexes have activity towards $S$. typhi and more effectively against $B$. cereus but Schiff base does not show activity against these bacterial strains. Schiff base and its complexes show almost similar activity against fungal strains.

Table 4. Minimum Inhibitory Concentration, MIC, $\mu \mathrm{g} / \mathrm{mL}$; (-) Resistant

\begin{tabular}{|c|c|c|c|c|c|c|c|c|c|}
\hline \multirow[b]{2}{*}{ 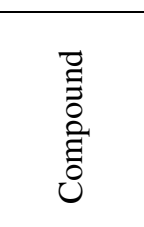 } & \multicolumn{6}{|c|}{ Bacteria strains } & \multicolumn{3}{|c|}{ Fungal strains } \\
\hline & $\begin{array}{c}S . \\
\text { aureus } \\
\text { (ATCC } \\
11632 \text { ) }\end{array}$ & $\begin{array}{l}\text { S. typhi } \\
\text { (ATCC } \\
15499 \text { ) }\end{array}$ & $\begin{array}{c}P . \\
\text { aeruginosa } \\
\text { (ATCC } \\
\text { 23564) }\end{array}$ & $\begin{array}{c}E . \\
a \quad c o l i \\
(\mathrm{ATCC} \\
35218)\end{array}$ & $\begin{array}{c}\text { B. cereus } \\
\text { (MTCC } \\
7350)\end{array}$ & $\begin{array}{c}P . \\
\text { rettgeri } \\
\text { (DRDE } \\
\text { strain) }\end{array}$ & A. niger & $\begin{array}{c}A . \\
\text { fumigates }\end{array}$ & $\begin{array}{l}\text { A. } \\
\text { flavus }\end{array}$ \\
\hline Sal-TMP & 2.5 & - & 1.25 & 5.0 & - & - & 20 & 5.0 & 1.25 \\
\hline I & 2.5 & - & 1.25 & - & 0.625 & 5.0 & - & - & - \\
\hline II & 5.0 & - & 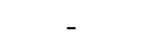 & - & 1.25 & 2.5 & 20 & 5.0 & 1.25 \\
\hline III & - & 20 & 10 & 5.0 & 1.25 & - & 5.0 & - & - \\
\hline IV & 1.25 & 2.5 & 1.25 & 5.0 & - & - & 5.0 & 10 & 5.0 \\
\hline V & - & 20 & 10 & 5.0 & 1.25 & - & 20 & - & - \\
\hline VI & 1.25 & 2.5 & 1.25 & 5.0 & - & - & - & 5.0 & - \\
\hline VII & 1.25 & - & 5.0 & 1.25 & 0.625 & 5.0 & - & 10 & - \\
\hline VIII & 2.5 & - & 1.25 & 5.0 & - & - & 20 & 5.0 & 1.25 \\
\hline
\end{tabular}

\section{Conclusion}

Aryltellurium(IV) and diaryltellurium(IV) dichlorides upon reaction with Schiff base(Sal-TMP) derived from salicylaldehyde and trimethoprim yield new complexes of tellurium(IV). The synthesized complexes were characterized by elemental analyses, conductance measurement, IR and ${ }^{1} \mathrm{H}$ NMR spectral studies. The analytical data suggest that the Sal-TMP Schiff base complexes have 1:1 stoichiometry. The Schiff bases (Sal-TMP) in these complexes functions as a uninegative tridentate ligand through azomethine nitrogen, phenolic oxygen after deprotonation and pyrimidine ring nitrogen atoms. Based on these studies, distorted octahedral geometry with two chelating rings has been assigned to these complexes. The complexes have been observed to possess substantial antimicrobial activity especially against bacteria.

\section{Acknowledgement}

The authors are grateful to M. D. University, Rohtak for providing the necessary facilities. One of the authors (Deepak) is also thankful to UGC New Delhi for providing fellowship. We also thank SAIF, Panjab University Chandigarh for providing the CHN analyses and spectral data.

\section{References}

1. Burchall J J, Corcoran J W and Hahn F E, Eds., Antibiotics, Springer, New York, NY, USA, $1975,3$.

2. Iqbal M S, Khan A H, Loothar B A and Bukhari I H, Med Chem Rev., 2009, 18, 31-42.

3. Blower P J, Аnnu Rep Prog Chem Sect A, 1999, 95, 631-655; DOI:10.1039/A808038K

4. Dhahir S A, Al-Sahib S A, Al Razaq W A and Kadhim N J, Baghdad Sci J., 2010, 7(1), 621.

5. Dr. Karl Thomae Gmb H, (Biberach an der Riss. DE), United States Patent. 4829058.

6. EL-Mossalamy E H, AL-Thabati S A, ALNomaiser F M, Commun Fac Sci Univ Ank Series B, 2005, 51(2), 21-30 
7. Sinha D, Tiwari A K, Singh S, Shukla G, Mishra P, Chandra H and Mishra A K, Eur J Med Chem., 2008, 43(1), 160-165; DOI:10.1016/j.ejmech.2007.03.022

8. Crowe A J, Smith P J and Atassi G, Chem Biol Interact., 1980, 32(1-2), 171-178; DOI:10.1016/0009-2797(80)90075-7

9. Wang M, Wang L F, Li Y Z, Li Q X, Xu Z D and Qu D M, Trans Met Chem., 2001, 26(3), 307-310; DOI:10.1023/A:1007159301849

10. Zishen Wu, Ziqi Gui and Zhenhuan Yen, Synth React Inorg Met Org Chem., 1990, 20(3), 335-344.

11. Desai S B, Desai P B and Desai K R, Hetrocycl Commun., 2001, 7(1), 83-90; DOI:10.1515/HC.2001.7.1.83

12. Dhumwad S D, Gudasi K B and Goudar T R, Indian J Chem., 1994, 33A, 320-324.

13. Przybylski P, Huczynski A, Pyta K, Brzezinski B and Bartl F, Curr Org Chem., 2009, 13(2), 124-148; DOI:10.2174/138527209787193774

14. Pandeya S N, Sriram D, Nath G and De Clercq E, Pharm Acta Hely., 1999, 74(1), 7-11.

15. Karthikeyan M S, Parsad D J, Poojary B, Bhat K S, Holla B S and Kumari N S, Bioorg. Med. Chem., 2006, 14(22), 7482-7489; DOI:10.1016/j.bmc.2006.07.015

16. Holla B S, Akberali P M and Shivananda M K, II Farmaco, 2001, 56(12), 919-927; DOI:10.1016/S0014-827X(01)01124-7

17. Jarrahpour A, Khalili D, De Clercq E, Salmi C and Brunel J M, Molecules, 2007, 12(8), 1720-1730; DOI:10.3390/12081720

18. Da Silva C M, da Silva D L, Modolo L V, Alves R B, de Resende, M A, Martins C V B and de Fatima A J, Adv Res., 2011, 2, 1-8; DOI:10.1016/j.jare.2010.05.004

19. Singh H, Yadav L D S and Mishra S B S, J Inorg Nucl Chem., 1981, 43(7), 17011704; DOI:10.1016/0022-1902(81)80367-3

20. Saravanan G, Pannerselvam P and Prakash C R, J Adv Pharm Techn Res., 2010, 1(3), 320-325; DOI:10.4103/0110-5558.72426

21. Panneerselvam P, Nair R R, Vijayalakshmi G, Subramanian E H and Sridhar S K, Eur. J. Med. Chem. 2005, 40, 225-229; DOI:10.1016/j.ejmech.2004.09.003

22. Singh N K and Singh S B, Indian J Chem., 2001, 40A, 1070-1075.

23. Walsh O M, Meegan M J, Prendergast R M, Nakib T A, Eur J Med Chem., 1996, 31, 989-1000; DOI:10.1016/S0223-5234(97)86178-8

24. Odabasoglu M, Arslan F, Ölmez H and Büyükgüngör O, Dyes and Pigments, 2007, 75(3), 507-515; DOI:10.1016/j.dyepig.2006.06.033

25. Vicini P, Geronikaki A, Incerti M, Busonera B, Poni G, Cabras C A and Colla P L, Bioorg Med Chem., 2003, 11(23), 4785-4789; DOI:10.1016/S09680896(03)00493-0

26. Pandeya S N, Sriram D, Nath G and DeClercq E, Eur J Pharm Sci., 1999, 9, 25-31.

27. Samadhiya S and Halve A, Orient J Chem., 2001, 17, 119-122.

28. Celik Ö, Ulusoy M, Tas E and Ide S, Anal Sciences, 2007, 23, x185.

29. Mahmoud M R, El-Gyar S A, Mousrafa A A and Shaker A, Polyhedron, 1987, 6(5), 1017-1020; DOI:10.1016/S0277-5387(00)80947-X

30. Qing-Yu H, Zheng-Hua M and Ya-Me Z, J Coord Chem., 1990, 21(3), 199-207; DOI:10.1080/00958979009409716

31. Cozzi P G, Chem Soc Rev., 2004, 33, 410-421; DOI:10.1039/B307853C

32. Tarafder M T H, Jin KT, Crouse K A, Ali A M. Yamin, B $M$ and Fun H K, Polyhedron, 2002, 21(25-26), 2547-2554; DOI:10.1016/S0277-5387(02)01188-9

33. Pandeya S N, Sriram D, Clercq E DE., Pannecouque C and Witvrouw M, Indian J Pharm Sci., 1998, 60(4), 207-212. 
34. Fleischman S H and Brooks C L, Proteins: Structure, Function and Genetics, 1990, 7, 52-61.

35. Suling W J, Reynolds R C, Barrow E W, Wilson L N, Piper J R and Barrow W W, J Antimicrobial Chemother., 1998, 42(6), 811-815; DOI:10.1093/jac/42.6.811

36. Gulcan M, Sonmez M and Berber I, Turkey J Chem., 2012, 36(1), 189-200.

37. Raman N, Kulandaisamy A and Jeyasubramanian K, Synth React Inorg Met Org Chem., 2001, 31(7), 1249-1270.

38. Wynne K J and Pearson P S, Inorg Chem., 1971, 10(12), 2735-2739; DOI:10.1021/ic50106a022

39. Wynne K J and Pearson P S, J Chem Soc Commun., 1970, 556-557; DOI:10.1039/C2970000556B

40. Wynne K J, Clark A J and Berg M, J Chem Soc Dalton Trans., 1972, 2370-2374; DOI:10.1039/DT9720002370

41. Clark E R, Collet A J and Naik D G, J Chem Soc Dalton Trans., 1973, 1961-1962; DOI:10.1039/DT9730001961

42. Berg M C, Diss Abstr Int., 1972, 33, 2982.

43. Srivastava T N, Singh M and Singh H B, Indian J Chem., 1982, 21A, 307-309.

44. Srivastava T N, Srivastava R C and Srivastava M, Indian J Chem., 1982, 21A, 539.

45. Srivastava T N, Srivastava R C and Srivastava V K, J Indian Chem Soc., 1983, 60, 891-892.

46. Garad M V, Polyhedron, 1985, 4(8), 1353-1355; DOI:10.1016/S0277-5387(00)86963-6

47. Verma K K and Reena, Synth React Inorg Met-Org Chem., 1999, 29(3), 499-512; DOI:10.1080/00945719909349465

48. Verma K K, Dahiya R and Soni D, Synth React Inorg Met Org Chem., 1999, 29(6), 1033-1052; DOI:10.1080/00945719909349509

49. Verma K K and Dahiya R, Synth React Inorg Met Org Chem., 1999, 29(7), 12991314; DOI:10.1080/00945719909349529

50. Verma K K and Reena, Phosphorus, Sulfur Silicon Related Elements, 1999, 148(1), 227-234; DOI:10.1080/10426509908037013

51. Verma K K and Seema, Int J Chem Sci., 2008, 6, 371-380.

52. Srivastava S, Soni D K and Gupta H S, J Indian Chem Soc., 1996, 73, 255.

53. Narwal J K, Chhabra S, Malik R K, Garg S and Verma K K, Oriental J Chem., 2013, 29, 1339-1349.

54. Chhabra S and Verma K K, J Chem Pharm Res., 2010, 2(4), 569-575.

55. Vogel A I, A Test Book of Organic Chemistry, $3^{\text {rd }}$ Edn., Longman, London, 1975.

56. Weissberger A, Ed., Technique of Organic Chemistry, Vol. 7, $2^{\text {nd }}$ Edn., Interscience Publishers, Inc. N. Y., 1967.

57. Morgan G T and Kellet R E, J Chem Soc., 1926, 1080-1088; DOI:10.1039/JR9262901080

58. Petragnani $\mathrm{N}$ and Stefani $\mathrm{H}$ A, Tellurium in Organic Chemistry, $2^{\text {nd }}$ Edn., Academic Press, London, 2007, 67, 76.

59. Bergman J, Tetrahedron, 1972, 28(12), 3323-3331; DOI:10.1016/S0040-4020(01)93674-9

60. Khandelwal B L, Kumar K and Berry F J, Inorg Chim Acta, 1981, 99(2), 135-137; DOI:10.1016/S0020-1693(00)87958-X

61. Berry F J, Kustan E H, Roshani M and Smith B C, J Organometal Chem., 1975, 99(1), 115-117; DOI:10.1016/S0022-328X(00)86367-6

62. Khandelwal B L, Kumar K and Raina K, Synth React Inorg Met Org Chem., 1981, 11, 65-78.

63. Geary W J, Coord Chem Rev., 1971, 7(1), 81-122; DOI:10.1016/S0010-8545(00)80009-0 
64. Greenwood N N, Straughan B P and Wilson A E, J Chem Soc A, 1968, 2209-2212; DOI: $10.1039 / \mathrm{J} 19680002209$

65. Srivastava K P, Singh A and Singh S K, IOSR J Appl Chem., 2014, 7(4), 16-23; DOI:10.9790/5736-07411623

66. Baker A W and Shulgin A T, J Am Chem Soc., 1959, 81(7), 1523-1529; DOI:10.1021/ja01516a001

67. Freedman H H, J Am Chem Soc., 1961, 83(13), 2900-2905; DOI:10.1021/ja01474a026

68. Flett M St C, Spectrochim Acta, 1957, 10(1), 21-37; DOI:10.1016/0371-1951(57)80160-X

69. Bellamy I J, Infrared Spectra of Complex Molecules, Chapman and Hall Ltd. London, 1975.

70. Matijević-Sosa J, Vinković M and Vikić-Topić D, Croatica Chemica Acta., 2006, 79(3), 489-495.

71. Mishra A P and Soni M, Metal Baesd Drugs, 2008, 71, 243; DOI:10.1155/2008/875410

72. Aminabhavi T M, Biradar N S and Patil C S, Inorganica Chimica Acta., 1983, 78, 107-111; DOI:10.1016/S0020-1693(00)86498-1

73. Biradar N S, Aminabhavi T M and Patil C S, Inorganica Chimica Acta., 1983, 78, 47-50; DOI:10.1016/S0020-1693(00)86487-7

74. Tumer M, Celik C, Koksal H and Serin S, Transition Metal Chem., 1999, 24(5), 525532; DOI:10.1023/A:1006982622965

75. Gwaram N S, Mohd Ali H, Khaledi H, Abdulla M A, Hamid A, Hadi A, Kawi Lin T, Ching C L and Ooi C L, Molecules, 2012, 17(5), 5952;

DOI:10.3390/molecules17055952

76. Rudzinski W E and Aminabhavi T M, Inorganica Chimica Acta., 1982, 67, 177-182; DOI:10.1016/S0020-1693(00)85061-6

77. Osowole A A, Wakil S M and Alao O K, World Appl Sci J., 2015, 33(2), 336-342; DOI:10.5829/idosi.wasj.2015.33.02.22206

78. UR-Rehman S, Faiz A and Nawaz R, J Chil Chem Soc., 2013, 58(1), 1624-1627; DOI:10.4067/S0717-97072013000100026.

79. Mohamed G G and Abd El-Wahab Z H, J Thermal Anal Calorimetry, 2003, 73(1), 347-359; DOI:10.1023/A:1025126801265

80. Verma K K, Soni D and verma, Phosphorus, Sulfur Silicon, 2000, 166(1), 231-241; DOI:10.1080/10426500008076544

81. Pant B C, McWhinnie W R and Dance N S, J Organmetal Chem., 1973, 63, 305-310; DOI:10.1016/S0022-328X(73)80043-9

82. Srivastava T N, Singh J D, Indian J. Chem., 1987, 26A, 260.

83. Chauhan S, Garg S and Verma K K, Chem Sci Trans., 2016, 5(2), 431-441; DOI:10.7598/cst2016.1193

84. Kulkarani Y D, Srivastava S, Abdi S H R and Athar M, Synth React Inorg Met Org Chem., 1985, 15(8), 1043-1059; DOI:10.1080/00945718508060634

85. Chauhan S, Garg S and Verma K K, Res J Pharm Biol Chem Sci., 2016, 7(2), 265-274.

86. Aminabhavi T M and Rudzinski W E, Inorganica Chimica Acta., 1983, 76, L131L134; DOI:10.1016/S0020-1693(00)81478-4

87. Aminabhavi T M and Rudzinski W E, Inorganica Chimica Acta., 1983, 78, 51-55; DOI:10.1016/S0020-1693(00)86488-9

88. Cappuccino J C, Sherman N, Microbiology- A Laboratory Manual, Addison Wesley, California, 1999, 263.

89. Pharmacopoeia of India, Volume 1, Controller of Publications, Ministry of Health Department, Government of India, New Delhi, 2007, 37. 


\title{
Synthesis, Spectral and Biological Studies of Some Salicylidene-Trimethoprim Schiff Base Complexes of Aryltellurium(IV)
}

\author{
DEEPAK, K. K. VERMA and SAPANA GARG* \\ Department of Chemistry, Maharshi Dayanand University, Rohtak-124001, India \\ sapanagarg1511@gmail.com
}

Received 14 February 2017 / Accepted 2 March 2017

\begin{abstract}
A novel monobasic tridentate Schiff base salicylidene-trimethoprim, Sal-TMP, synthesized from trimethoprim and salicylaldehyde, form stable complexes with aryltellurium(IV) trichlorides and diaryltellurium(IV) dichlorides of the type Sal-TMP.ArTeCl ${ }_{2}$ and $\mathrm{Sal}_{-} \mathrm{TMP}_{\mathrm{Ar}} \mathrm{AeCl}$ (where $\mathrm{Ar}=p$-methoxyphenyl, $p$-ethoxyphenyl, $p$-hydroxyphenyl and 3-methyl-4-hydroxyphenyl). These have been characterized by elemental analyses, molar conductance, IR and ${ }^{1} \mathrm{H}$ NMR spectroscopy. The spectral studies predict the bonding of ligand through phenolic oxygen of Schiff base after deprotonation, nitrogen of the azomethine group and pyrimidine nitrogen to give hexacoordinated tellurium(IV) complexes. The complexes have also been screened for their antimicrobial activities against various bacteria and fungi organisms.
\end{abstract}

Keywords: Salicylidene-trimethoprim Schiff base, Aryltellurium(IV), Diaryltellurium(IV), Antibacterial, Antifungal activities

\section{Introduction}

Trimethoprim and its derivatives are broad spectrum antimicrobial agents with anti-parasitic activity $^{1-6}$ and were first described by Roth and coworker ${ }^{5}$. Schiff bases, also known as azomethine due to presence of $-\mathrm{C}=\mathrm{N}$ - group, play important role in biological system, such as anticancer $^{7-12}$, antibacterial ${ }^{13-15}$, antiviral ${ }^{16-18}$, antifungal ${ }^{19-21}$ and other biological properties $^{22-27}$ and also have been extensively used as ligands in coordination chemistry because of their excellent donor abilities as chelating agent ${ }^{28-32}$. Medicinal chemists have reported new derivatives of trimethoprim(TMZ) ${ }^{33-35}$ including the Schiff base derived from salicylaldehyde $2-4,36,37$.

Also, aryltellurium(IV) chlorides are known ${ }^{38-54}$ to act as Lewis acids and form complexes with several N-, O- and S- donor bases. In view of this, we herein report some new complexes derived from aryltellurium(IV) trichlorides, $\mathrm{RTeCl}_{3}$ and diaryltellurium(IV) dichlorides, $\mathrm{R}_{2} \mathrm{TeCl}_{2}$ with salicylidene-trimethoprim Schiff base(Sal-TMP). 


\section{Experimental}

All preparations were carried out under dry $\mathrm{N}_{2}$ atmosphere and the solvents used were purified by standard method ${ }^{55,56}$ before use. The purity of compounds was checked by thin layer chromatography using silica gel-G (Merck). Melting points were determined in open capillary tube and are uncorrected.

Carbon, hydrogen and nitrogen analyses were obtained microanalytically from SAIF, Panjab University Chandigarh on a ThermoFinnigan CHNS analyser. Conductivity was measured in DMSO at $25 \pm 2{ }^{\circ} \mathrm{C}$ with dip type conductivity cell on a microprocessor based conductivity bridge type MICROSIL.

IR $\left(4000-400 \mathrm{~cm}^{-1}\right)$ and far IR $\left(400-50 \mathrm{~cm}^{-1}\right)$ spectra were recorded in $\mathrm{KBr} /$ polyethylene pellets on a FT-Infrared spectrophotometer model RZX (Perkin Elmer) at SAIF, Panjab University Chandigarh. Proton magnetic resonance spectra were recorded in DMSO- $\mathrm{d}_{6}$ using TMS as an internal reference on BRUKER AVANCE II 400 NMR spectrometer. The antimicrobial screening was carried out by tube dilution method at Department of Pharmaceutical Sciences, M. D. University, Rohtak, India.

\section{Preparation of aryltellurium(IV) trichlorides and diaryltellurium(IV) dichlorides}

p-Methoxyphenyltellurium(IV) $\quad$ trichloride $^{57,58}, \quad$ bis(p-methoxyphenyl)tellurium(IV) dichloride ${ }^{58,59}, \quad p$-ethoxyphenyltellurium (IV) trichloride ${ }^{60}$, bis( $p$-ethoxyphenyl)tellurium dichloride $^{60} p$-hydroxyphenyltellurium(IV) trichloride ${ }^{61}$, bis( $p$-hydroxyphenyl) tellurium(IV) dichloride $^{61}$, 3-methyl-4-hydroxyphenyltellurium(IV) trichloride $^{62}$ and bis(3-methyl-4hydroxyphenyl)tellurium(IV) dichloride ${ }^{62}$ were prepared by the reactions of $\mathrm{TeCl}_{4}$ with anisole, phenetole, phenol, $o$-cresol respectively, by the methods reported in the literature ${ }^{57-62}$.

\section{Preparation of salicylidene-trimethoprim Schiff base (Sal-TMP)}

Equimolar quantity of saturated methanolic solution of drug and salicylaldehyde were mixed thoroughly. To this mixture $0.1 \%$ methanolic $\mathrm{KOH}$ was added to adjust the $\mathrm{pH}$ of the solution between 7-8 and was refluxed for 2 hours. A clear yellowish-green coloured solution was obtained. After completion of the reaction, the Schiff base ligand was isolated by crystallization after volume reduction by evaporation. The crystalline product was filtered and dried under vacuum and kept in desiccator over $\mathrm{P}_{4} \mathrm{O}_{10}$ until further use. Yield $=$ $80 \%$, M.pt.(decomp.) $=188-190{ }^{\circ} \mathrm{C}$. Analysis (Calculated) $\mathrm{C}_{21} \mathrm{H}_{22} \mathrm{~N}_{4} \mathrm{O}_{4}: \mathrm{C}(63.95), \mathrm{H}(5.62)$ and $\mathrm{N}(14.20)$; Found: $\mathrm{C}(63.50), \mathrm{H}(5.27)$ and $\mathrm{N}(13.99)$.

\section{Preparation of salicylidene-trimethoprim complexes of aryltellurium(IV) trichlorides} and diaryltellurium(IV) dichlorides

Aryltellurium(IV) trichlorides, $\mathrm{ArTeCl}_{3}$ and diaryltellurium(IV) dichlorides $\mathrm{Ar}_{2} \mathrm{TeCl}_{2}$ (Ar= $p$-methoxyphenyl, $p$-ethoxyphenyl, $p$-hydroxyphenyl and 3-methyl-4-hydroxyphenyl), when reacted with sodium salt of salicylidene-trimethoprim in equimolar ratio, yield SalTMP.ArTeCl $\mathrm{Cl}_{2}$ and Sal-TMP.Ar $\mathrm{TeCl}_{2} \mathrm{Type}$ complexes.

Sodium salt of the ligand was prepared by reacting equimolar (1:1) quantity of sodium metal and Schiff base in methanol. The solvent was distilled off to obtain sodium salt of Schiff base. Then a methanolic saturated solution of $2 \mathrm{mmol}$ of aryltellurium(IV) trichloride or diaryltellurium(IV) dichloride was added dropwise to suspension of $2 \mathrm{mmol}$ of sodium salt of Schiff base in about $50 \mathrm{~mL}$ benzene under reflux. The reaction mixture was further refluxed for 3-4 hours, cooled and precipitated sodium chloride was filtered off. The filtrate was then concentrated to about one third of original volume under reduced pressure and cooled in an ice bath to obtain coloured product. This was filtered, washed with benzene + methanol (1:1) and dried in vacuum desiccator over $\mathrm{P}_{4} \mathrm{O}_{10}$. 


\section{Results and Discussion}

$\mathrm{TeCl}_{4}$ when heated with anisole ${ }^{57-59}$, phenetole ${ }^{60}$, phenol ${ }^{61}, o$-cresol ${ }^{62}$ (Ar-H) appears to undergo Friedel-Crafts type condensation reaction whereby $\mathrm{TeCl}_{3}{ }^{+}$unit attacks a position para to the methoxy/ethoxy/hydroxy groups in the aromatic rings, thus resulting in the formation of aryltellurium(IV) trichlorides and diaryltellurium(IV) dichlorides.

$$
\begin{gathered}
\mathrm{Ar}-\mathrm{H}+\mathrm{TeCl}_{4} \longrightarrow \mathrm{ArTeCl}_{3}+\mathrm{HCl} \\
2 \mathrm{Ar}-\mathrm{H}+\mathrm{TeCl}_{4} \longrightarrow \mathrm{Ar}_{2} \mathrm{TeCl}_{2}+2 \mathrm{HCl}
\end{gathered}
$$

Preparation of salicylidene-trimethoprim Schiff base (Sal-TMP) by the reaction of trimethoprim drug and salicylaldehyde can be represented by following equations.

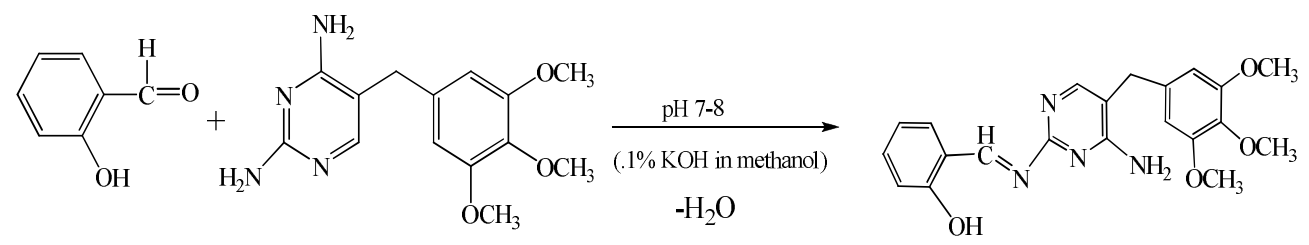

Sodium salt of salicylidene-trimethoprim Schiff base (Sal-TMP) reacts with aryltellurium(IV) trichlorides and diaryltellurium(IV) dichlorides in 1:1 molar ratio to yield the corresponding aryltellurium(IV) complexes.

$$
\begin{aligned}
& \mathrm{Sal}-\mathrm{TMP}+\mathrm{ArTeCl}_{3} \underset{\mathrm{NaCl}}{\stackrel{\mathrm{Na} / \mathrm{CH}_{3} \mathrm{OH}}{\longrightarrow}} \text { (Sal-TMP).ArTeCl } 2 \\
& \mathrm{Sal}-\mathrm{TMP}+\mathrm{Ar}_{2} \mathrm{TeCl}_{2} \underset{-\mathrm{NaCl}}{\stackrel{\mathrm{Na} / \mathrm{CH}_{3} \mathrm{OH}}{\longrightarrow}} \text { (Sal-TMP). } \mathrm{Ar}_{2} \mathrm{TeCl}_{2}
\end{aligned}
$$

All the tellurium(IV) complexes are coloured, crystalline solids, stable at room temperature and non-hygroscopic in nature. The complexes have been analysed for their tellurium, chlorine, carbon, hydrogen and nitrogen contents and the data along with their physical properties and yields are presented in Table 1.

\section{Conductance studies}

Molar conductance $\left(\Lambda_{M}\right)$ data for the complexes in DMSO are complied in Table 1. The $\Lambda_{M}$ value at $c a .10^{-3} \mathrm{M}$ for aryltellurium(IV) complexes in DMSO lie in the range 27.36-91.83 S $\mathrm{cm}^{2} \mathrm{~mol}^{-1}$ which predict the non electrolyte to $1: 1$ electrolyte $^{63,64}$ type behaviour of these complexes in DMSO, probably due to ionization into $\mathrm{ArTeCl}$.Sal- $\mathrm{TMP}^{+} / \mathrm{Ar}_{2} \mathrm{Te}$.Sal-TMP ${ }^{+}$ and $\mathrm{Cl}^{-}$in DMSO. This conductance behavoiur of tellurium(IV) salicylidene-trimethoprim Schiff base complexes is different from those of transition metal complexes ${ }^{65}$, which are reported to be non-electrolytes. The higher $\Lambda_{\mathrm{M}}$ values for some complexes may be due to steric factors and donor behaviour of DMSO to result in probable dissociation into solvated cation and $\mathrm{L}^{-}$along with $\mathrm{Cl}^{-}$in DMSO.

\section{Infrared spectra}

The IR data of Schiff base and its tellurium(IV) complexes are listed in Table 2. The spectra of Sal-TMP Schiff base complexes are quite complex and an attempt has therefore been made to identify the donor sites by comparing the spectra of complexes with parent ligand and $\mathrm{ArTeCl}_{3} / \mathrm{Ar}_{2} \mathrm{TeCl}_{2}$. 
Table 1. Analytical data, molar conductance and physical properties of salicylidene-trimethoprim Schiff base (Sal-TMP) complexes of tellurium(IV)

\begin{tabular}{|c|c|c|c|c|c|c|c|c|c|c|}
\hline \multirow[b]{2}{*}{$\begin{array}{l}\text { Compound } \\
\text { No. }\end{array}$} & \multirow[b]{2}{*}{$\begin{array}{l}\text { Complex } \\
\text { (Ar) }\end{array}$} & \multirow{2}{*}{$\begin{array}{c}\text { Empirical } \\
\text { formula } \\
\text { (Formula Wt.) }\end{array}$} & \multirow[b]{2}{*}{$\begin{array}{l}\text { Colour } \\
\text { (Yield, \%) }\end{array}$} & \multirow{2}{*}{$\begin{array}{l}\text { M. Pt. } \\
{ }^{\circ} \mathrm{C} \\
\text { dec. }\end{array}$} & \multicolumn{5}{|c|}{ Analyses \% Found (Calculated) } & \multirow{2}{*}{$\begin{array}{l}\Lambda_{\mathrm{M}} \text { at } c a \\
10^{-3} \mathrm{M} \mathrm{S} \\
\mathrm{cm}^{2} \mathrm{~mol}^{-1} \\
\text { in DMSO }\end{array}$} \\
\hline & & & & & $\mathrm{C}$ & $\mathrm{H}$ & $\mathrm{N}$ & $\mathrm{Te}$ & $\mathrm{Cl}$ & \\
\hline $\begin{array}{l}\text { Schiff } \\
\text { Base }\end{array}$ & Sal-TMP & $\mathrm{C}_{21} \mathrm{H}_{22} \mathrm{~N}_{4} \mathrm{O}_{4}$ & $\begin{array}{l}\text { Yellowish } \\
\text {-green }(80)\end{array}$ & $188-190$ & $\begin{array}{c}63.50 \\
(63.95)\end{array}$ & $\begin{array}{c}5.27 \\
(5.62)\end{array}$ & $\begin{array}{l}13.99 \\
(14.20)\end{array}$ & - & - & - \\
\hline I & $\begin{array}{l}\text { (Sal-TMP).ArTeCl }{ }_{2} \\
\text { (p-methoxyphenyl) }\end{array}$ & $\begin{array}{c}\mathrm{C}_{28} \mathrm{H}_{28} \mathrm{Cl}_{2} \mathrm{~N}_{4} \mathrm{O}_{5} \mathrm{Te} \\
(699.05)\end{array}$ & $\begin{array}{l}\text { Light cream } \\
(92)\end{array}$ & $230-232$ & $\begin{array}{c}47.88 \\
(48.11)\end{array}$ & $\begin{array}{c}3.99 \\
(4.04)\end{array}$ & $\begin{array}{c}7.75 \\
(8.01)\end{array}$ & $\begin{array}{c}18.07 \\
(18.24)\end{array}$ & $\begin{array}{c}9.89 \\
(10.01)\end{array}$ & 53.19 \\
\hline II & $\begin{array}{c}\text { (Sal-TMP).ArTeCl } \\
\text { (p-ethoxyphenyl) }\end{array}$ & $\begin{array}{c}\mathrm{C}_{29} \mathrm{H}_{30} \mathrm{Cl}_{2} \mathrm{~N}_{4} \mathrm{O}_{5} \mathrm{Te} \\
(713.08)\end{array}$ & $\begin{array}{c}\text { Cream } \\
(85)\end{array}$ & $258-260$ & $\begin{array}{c}48.12 \\
(48.85)\end{array}$ & $\begin{array}{c}3.87 \\
(4.24)\end{array}$ & $\begin{array}{l}7.52 \\
(7.86)\end{array}$ & $\begin{array}{c}17.38 \\
(17.89)\end{array}$ & $\begin{array}{c}9.52 \\
(9.81)\end{array}$ & 52.88 \\
\hline III & $\begin{array}{c}(\mathrm{Sal}-\mathrm{TMP}) \cdot \mathrm{ArTeCl}_{2} \\
(p \text {-hydroxyphenyl })\end{array}$ & $\begin{array}{c}\mathrm{C}_{27} \mathrm{H}_{26} \mathrm{Cl}_{2} \mathrm{~N}_{4} \mathrm{O}_{5} \mathrm{Te} \\
(685.03)\end{array}$ & $\begin{array}{l}\text { Dark cream } \\
\quad(94)\end{array}$ & $208-210$ & $\begin{array}{c}46.78 \\
(47.34)\end{array}$ & $\begin{array}{c}3.50 \\
(3.83)\end{array}$ & $\begin{array}{c}7.88 \\
(8.18)\end{array}$ & $\begin{array}{c}18.28 \\
(18.63)\end{array}$ & $\begin{array}{c}9.98 \\
(10.21)\end{array}$ & 55.73 \\
\hline IV & $\begin{array}{c}\text { (Sal-TMP).ArTeCl } \\
\text { (3-methyl-4- } \\
\text { hydroxyphenyl) }\end{array}$ & $\begin{array}{c}\mathrm{C}_{28} \mathrm{H}_{28} \mathrm{Cl}_{2} \mathrm{~N}_{4} \mathrm{O}_{5} \mathrm{Te} \\
(699.05)\end{array}$ & $\begin{array}{l}\text { Light } \\
\text { cream } \\
(80)\end{array}$ & $198-200$ & $\begin{array}{c}47.58 \\
(48.11)\end{array}$ & $\begin{array}{c}3.84 \\
(4.04)\end{array}$ & $\begin{array}{c}7.80 \\
(8.01)\end{array}$ & $\begin{array}{c}18.15 \\
(18.24)\end{array}$ & $\begin{array}{c}9.85 \\
(10.01)\end{array}$ & 58.68 \\
\hline $\mathrm{V}$ & $\begin{array}{c}(\mathrm{Sal}-\mathrm{TMP}) \cdot \mathrm{Ar}_{2} \mathrm{TeCl} \\
(p \text {-methoxyphenyl })\end{array}$ & $\begin{array}{c}\mathrm{C}_{35} \mathrm{H}_{35} \mathrm{ClN}_{4} \mathrm{O}_{6} \mathrm{Te} \\
(770.73)\end{array}$ & $\begin{array}{c}\text { Pale yellow } \\
\text { (84) }\end{array}$ & $130-132$ & $\begin{array}{c}53.84 \\
(54.54)\end{array}$ & $\begin{array}{c}4.31 \\
(4.58)\end{array}$ & $\begin{array}{c}7.11 \\
(7.27)\end{array}$ & $\begin{array}{c}16.43 \\
(16.56)\end{array}$ & $\begin{array}{c}4.42 \\
(4.60)\end{array}$ & 91.83 \\
\hline VI & $\begin{array}{c}\text { (Sal-TMP).Ar }{ }_{2} \mathrm{TeCl} \\
(p \text {-ethoxyphenyl) }\end{array}$ & $\begin{array}{c}\mathrm{C}_{37} \mathrm{H}_{39} \mathrm{ClN}_{4} \mathrm{O}_{6} \mathrm{Te} \\
(798.78)\end{array}$ & $\begin{array}{l}\text { Light yellow } \\
\text { (86) }\end{array}$ & $150-152$ & $\begin{array}{c}54.84 \\
(55.63)\end{array}$ & $\begin{array}{c}4.53 \\
(4.92)\end{array}$ & $\begin{array}{c}6.84 \\
(7.01)\end{array}$ & $\begin{array}{c}15.50 \\
(15.97)\end{array}$ & $\begin{array}{c}4.30 \\
(4.44)\end{array}$ & 35.90 \\
\hline VII & $\begin{array}{l}\text { (Sal-TMP).Ar } \mathrm{Ar}_{2} \mathrm{TeCl} \\
(p \text {-hydroxyphenyl) }\end{array}$ & $\begin{array}{c}\mathrm{C}_{33} \mathrm{H}_{31} \mathrm{ClN}_{4} \mathrm{O}_{6} \mathrm{Te} \\
(742.68)\end{array}$ & $\begin{array}{l}\text { Red } \\
(89)\end{array}$ & $146-148$ & $\begin{array}{c}52.84 \\
(53.37)\end{array}$ & $\begin{array}{c}4.01 \\
(4.21)\end{array}$ & $\begin{array}{c}7.27 \\
(7.54)\end{array}$ & $\begin{array}{c}16.89 \\
(17.18)\end{array}$ & $\begin{array}{c}4.52 \\
(4.77)\end{array}$ & 36.00 \\
\hline VIII & $\begin{array}{c}\text { (Sal-TMP). } \mathrm{Ar}_{2} \mathrm{TeCl} \\
\text { (3-methyl-4- } \\
\text { hydroxyphenyl) }\end{array}$ & $\begin{array}{c}\mathrm{C}_{35} \mathrm{H}_{35} \mathrm{ClN}_{4} \mathrm{O}_{6} \mathrm{Te} \\
(770.73)\end{array}$ & $\begin{array}{l}\text { Brown } \\
(78)\end{array}$ & $140-142$ & $\begin{array}{l}53.80 \\
(54.54)\end{array}$ & $\begin{array}{c}4.34 \\
(4.58)\end{array}$ & $\begin{array}{c}7.09 \\
(7.27)\end{array}$ & $\begin{array}{c}16.45 \\
(16.56)\end{array}$ & $\begin{array}{c}4.32 \\
(4.60)\end{array}$ & 27.36 \\
\hline
\end{tabular}


Table 2. Important infrared absorption bands $\left(\mathrm{cm}^{-1}\right)$ of Schiff base (Sal-TMP) and complexes

\begin{tabular}{|c|c|c|c|c|c|c|c|}
\hline Compound & $v_{(O-}$ & $v_{(1}$ & $v_{(C}$ & $v_{(C}$ & $v_{(\mathrm{C}-}$ & $v_{(\mathrm{Te}-\mathrm{N})}$ & $v_{(\mathrm{Te}}$ \\
\hline Sal-TMP & $2836 \mathrm{w}$ & $3317 \mathrm{~m} \mathrm{3119} \mathrm{m}$ & $1636 \mathrm{sh}$ & $1633 \mathrm{w} 1593 \mathrm{~s}$ & $1263 \mathrm{~s}$ & - & - \\
\hline $\bar{I}$ & - & $3323 \mathrm{~m} 3184 \mathrm{~m}$ & $1674 \mathrm{mb}$ & $1644 \mathrm{mb} 1587 \mathrm{~s}$ & $1341 \mathrm{~s}$ & $415 \mathrm{~m}$ & $288 \mathrm{w}$ \\
\hline II & - & $3323 \mathrm{~m} 3170 \mathrm{~m}$ & $1647 \mathrm{sh}$ & **1586 s & $1304 \mathrm{~s}$ & $420 \mathrm{~m}$ & $295 w$ \\
\hline III & 3405 m* & $3323 \mathrm{~m} 3150 \mathrm{~m}$ & $1674 \mathrm{mb}$ & $1641 \mathrm{mb} 1586 \mathrm{~s}$ & $1341 \mathrm{~s}$ & $419 \mathrm{~s}$ & $270 \mathrm{w}$ \\
\hline IV & 3398 m* & $3319 \mathrm{~m} 3172 \mathrm{~m}$ & $1652 \mathrm{mb}$ & 1649 & $1333 \mathrm{~s}$ & $450 \mathrm{~s}$ & $277 \mathrm{w}$ \\
\hline V & - & $3325 \mathrm{~m}$ & 164 & $* * 1$ & $1333 \mathrm{~s}$ & $416 \mathrm{~s}$ & $290 \mathrm{w}$ \\
\hline VI & - & $3320 \mathrm{~m} 3158 \mathrm{~m}$ & $1640 \mathrm{sh}$ & $* * 1590 \mathrm{~s}$ & $1331 \mathrm{~s}$ & $410 \mathrm{~m}$ & $273 w$ \\
\hline VII & $3401 \mathrm{w}^{*}$ & $3324 \mathrm{~m} 3168 \mathrm{~m}$ & $1674 \mathrm{mb}$ & $1643 \mathrm{mb} 1584 \mathrm{~s}$ & $1340 \mathrm{~s}$ & $418 \mathrm{~m}$ & $285 \mathrm{w}$ \\
\hline VIII & $3463 \mathrm{w}^{*}$ & $3304 \mathrm{~m} 3106 \mathrm{~m}$ & $1634 \mathrm{sh}$ & $1643 \mathrm{mb} 1584 \mathrm{~s}$ & $1340 \mathrm{~s}$ & $422 \mathrm{~m}$ & $287 \mathrm{w}$ \\
\hline
\end{tabular}

$s=$ sharp, m=medium, mb=medium broad, sh=shoulder, $w=w e a k$, "Due to phenolic $\mathrm{OH}$ of Rte and $\mathrm{R}_{2} \mathrm{Te}$ moieties; ${ }^{* *}$ band not resolved due to overlapping of band $\boldsymbol{v}_{(C=N)}$

Examination of the Schiff base (Sal-TMP) spectrum shows the presence of the hydrogen bonded conjugated- chelate ring system ${ }^{66-68}$ with its centre at $\sim 2832 \mathrm{~cm}^{-1}$. Thus intramolecular $\mathrm{H}$ - bonding is occurring by means of the formation of a quasi six- membered ring involving the $\mathrm{OH}----\mathrm{N}=\mathrm{C}$ bond.<smiles>C1=Cc2ccccc2O[NH+]=1</smiles>

Figure 1. Hydrogen bonding

Thus band disappear on chelation with aryltellurium(IV) chlorides ${ }^{69}$. Hydrogen bond contributes to planarity of the molecule which helps in chelation ${ }^{70}$. Also an intense ligand band at $1263 \mathrm{~cm}^{-1}$ (phenolic -C-O) in free ligand has shifted to higher frequency side in complexes. All these indicate that the hydroxyl group of salicylaldehyde of Schiff base is involved in coordination with tellurium ${ }^{71-73}$.

In addition, the spectra of the Schiff base shown shoulder at $1636 \mathrm{~cm}^{-1}$ with slightly resolved weak band at $1633 \mathrm{~cm}^{-1}$ and sharp band at $1593 \mathrm{~cm}^{-1}$ assigned to $v_{(\mathrm{C}=\mathrm{N})}$ mode for vibration of azomethine group ${ }^{2,4,65,74-76}$ and $v_{(\mathrm{C}=\mathrm{N}) \text { pyrim. For pyrimidine ring }}{ }^{4,77}$. These shift in aryltellurium Schiff base complexes towards higher and lower value ${ }^{74-79}$ reflecting that ligand coordinate through nitrogen atom of azomethine and pyrimidine ring.

The medium intensity band at $3317 \mathrm{~cm}^{-1}$ and $3119 \mathrm{~cm}^{-1}$ due to $v_{(\mathrm{N}-\mathrm{H})}$ asymmetric and symmetric vibrations respectively indicate the non involvement of the nitrogen atom of $\mathrm{NH}_{2}$ group attached to pyrimidine in coordination. The appearance of new weak bands around $270-295 \mathrm{~cm}^{-1}$ due to $v_{(\mathrm{Te}-\mathrm{O})}^{80-83}$ mode and medium to strong band in the range of 410-422 $\mathrm{cm}^{-1}$ due to $v_{(\mathrm{Te}-\mathrm{N})}$ mode ${ }^{84}$ further supports the involvement of phenolic oxygen (after deprotonation), azomethine and pyrimidine nitrogen atoms of Schiff base in the coordination.

Thus, IR data predict the tridentate nature of Sal-TMP involving azomethine nitrogen atom, phenolic oxygen after deprotonation and pyrimidine ring nitrogen giving rise to twosix and four membered chelate rings with the tellurium centre. 


\section{${ }^{1}$ H NMR spectra}

In order to identify the solution structure of Schiff base (Sal-TMP) and its complexes, ${ }^{1} \mathrm{H}$ NMR spectra were recorded in DMSO- $\mathrm{d}_{6}$ and given in Table 3. The proton resonance of the $\mathrm{OH}$ group at $10.92 \delta \mathrm{ppm}^{76,85}$ in Schiff base due to presence of intramolecular hydrogen bonding ${ }^{74}$ disappear on complexation indicating the involvement of phenolic oxygen in the coordination via deprotonation ${ }^{76}$. The azomethine protons which resonate as a singlet at $10.02 \delta \mathrm{ppm}$, the coordination of azomethine nitrogen to tellurium in the complexes are clearly demonstrated by a downfield shift of the peak ${ }^{86}$.

Table 3. ${ }^{1} \mathrm{H}$ NMR spectral data of Schiff base (Sal-TMP) and complexes in DMSO- $\mathrm{d}_{6}$

\begin{tabular}{|c|c|}
\hline Compound & Chemical Shift, $\delta$ ppm \\
\hline Sal-TMP & $\begin{array}{l}2.525(\mathrm{~s}, 2 \mathrm{H}, \text { methylene }), \quad 3.551-3.745\left(\mathrm{~s}, \quad 9 \mathrm{H},-\mathrm{OCH}_{3}\right), \quad 5.672(\mathrm{~s}, 1 \mathrm{H}, \\
\text { pyrimidine }), 6.508\left(\mathrm{~s}, 2 \mathrm{H},-\mathrm{NH}_{2}\right), 7.526-8.169(\mathrm{~m}, 6 \mathrm{H}, \text { aromatic proton), } \\
10.021(\mathrm{~s}, 1 \mathrm{H}, \text { azomethine }), 10.921(\mathrm{~s}, 1 \mathrm{H}, \mathrm{Schiff} \text { base } \mathrm{OH})\end{array}$ \\
\hline I & $\begin{array}{l}2.539(\mathrm{~s}, 2 \mathrm{H}, \text { methylene }), 3.598-3.840\left(\mathrm{~s}, 12 \mathrm{H},-\mathrm{OCH}_{3}\right), 6.574(\mathrm{~s}, 1 \mathrm{H}, \\
\text { pyrimidine }), 6.926\left(\mathrm{~s}, 2 \mathrm{H},-\mathrm{NH}_{2}\right), 7.383-7.522(\mathrm{~m}, 10 \mathrm{H}, \text { aromatic proton }), \\
10.209(\mathrm{~s}, 1 \mathrm{H}, \text { azomethine })\end{array}$ \\
\hline II & $\begin{array}{l}1.335\left(\mathrm{t}, 3 \mathrm{H},-\mathrm{OCH}_{2} \mathrm{CH}_{3}\right), 2.546(\mathrm{~s}, 2 \mathrm{H}, \text { methylene }), 3.963\left(\mathrm{q}, 2 \mathrm{H},-\mathrm{OCH}_{2} \mathrm{CH}_{3}\right) \text {, } \\
3.515-3.875\left(\mathrm{~s}, 9 \mathrm{H},-\mathrm{OCH}_{3}\right), 6.540(\mathrm{~s}, 1 \mathrm{H}, \text { pyrimidine }), 6.950\left(\mathrm{~s}, 2 \mathrm{H},-\mathrm{NH}_{2}\right), \\
6.993-7.809(\mathrm{~m}, 10 \mathrm{H} \text {, aromatic proton), } 10.193(\mathrm{~s}, 1 \mathrm{H} \text {, azomethine })\end{array}$ \\
\hline III & $\begin{array}{l}2.548(\mathrm{~s}, 2 \mathrm{H}, \quad \text { methylene }), \quad 3.601-3.783\left(\mathrm{~s}, 9 \mathrm{H},-\mathrm{OCH}_{3}\right), \quad 6.569(\mathrm{~s}, 1 \mathrm{H}, \\
\text { pyrimidine }), 6.914\left(\mathrm{~s}, 2 \mathrm{H},-\mathrm{NH}_{2}\right), 7.370-7.787(\mathrm{~m}, 10 \mathrm{H}, \text { aromatic proton }), \\
8.155(\mathrm{~s}, 1 \mathrm{H}, \text { phenolic } \mathrm{OH} \text { of } \mathrm{RTe}), 10.201(\mathrm{~s}, 1 \mathrm{H}, \text { azomethine })\end{array}$ \\
\hline IV & $\begin{array}{l}2.536(\mathrm{~s}, 2 \mathrm{H}, \text { methylene }), 2.527\left(\mathrm{~s}, 3 \mathrm{H},-\mathrm{CH}_{3}\right), 3.587-3.770\left(\mathrm{~s}, 9 \mathrm{H},-\mathrm{OCH}_{3}\right) \text {, } \\
\text { 6.605(s, } 1 \mathrm{H}, \text { pyrimidine }), 6.918\left(\mathrm{~s}, 2 \mathrm{H},-\mathrm{NH}_{2}\right), 7.051-7.671(\mathrm{~m}, 9 \mathrm{H} \text {, aromatic } \\
\text { proton }), 8.251(\mathrm{~s}, 1 \mathrm{H} \text {, phenolic } \mathrm{OH} \text { of } \mathrm{RTe}), 10.239(\mathrm{~s}, 1 \mathrm{H} \text {, azomethine })\end{array}$ \\
\hline V & $\begin{array}{l}2.528(\mathrm{~s}, 2 \mathrm{H}, \text { methylene }), 3.673-3.877\left(\mathrm{~s}, 15 \mathrm{H},-\mathrm{OCH}_{3}\right), 6.516(\mathrm{~s}, 1 \mathrm{H} \text {, } \\
\text { pyrimidine }), 6.882\left(\mathrm{~s}, 2 \mathrm{H},-\mathrm{NH}_{2}\right), 6.910-7.804(\mathrm{~m}, 14 \mathrm{H}, \text { aromatic proton }) \\
10.186(\mathrm{~s}, 1 \mathrm{H}, \text { azomethine })\end{array}$ \\
\hline VI & $\begin{array}{l}\left.\text { 1.363(t, } 6 \mathrm{H},-\mathrm{OCH}_{2} \mathbf{C H}_{3}\right), 2.531(\mathrm{~s}, 2 \mathrm{H}, \text { methylene }), 4.019\left(\mathrm{q}, 4 \mathrm{H},-\mathrm{OCH}_{2} \mathrm{CH}_{3}\right) \text {, } \\
3.555-3.765\left(\mathrm{~s}, 9 \mathrm{H},-\mathrm{OCH}_{3}\right), 6.510(\mathrm{~s}, 1 \mathrm{H}, \text { pyrimidine }), 6.924\left(\mathrm{~s}, 2 \mathrm{H},-\mathrm{NH}_{2}\right) \text {, } \\
\text { 7.524-7.805(m, } 14 \mathrm{H} \text {, aromatic proton }), 10.216(\mathrm{~s}, 1 \mathrm{H} \text {, azomethine })\end{array}$ \\
\hline VII & $\begin{array}{l}2.545(\mathrm{~s}, 2 \mathrm{H}, \quad \text { methylene }), 3.568-3.926\left(\mathrm{~s}, 9 \mathrm{H},-\mathrm{OCH}_{3}\right), 6.554(\mathrm{~s}, 1 \mathrm{H} \text {, } \\
\text { pyrimidine }), 6.884\left(\mathrm{~s}, 2 \mathrm{H},-\mathrm{NH}_{2}\right), 7.488-7.678(\mathrm{~m}, 14 \mathrm{H}, \text { aromatic proton), } \\
8.249\left(\mathrm{~s}, 2 \mathrm{H}, \text { phenolic } \mathrm{OH} \text { of } \mathrm{R}_{2} \mathrm{Te}\right), 10.238(\mathrm{~s}, 1 \mathrm{H}, \text { azomethine })\end{array}$ \\
\hline VIII & $\begin{array}{l}2.538(\mathrm{~s}, 2 \mathrm{H}, \text { methylene }), 2.529\left(\mathrm{~s}, 6 \mathrm{H},-\mathrm{CH}_{3}\right), 3.555-3.750\left(\mathrm{~s}, 9 \mathrm{H},-\mathrm{OCH}_{3}\right) \text {, } \\
6.502(\mathrm{~s}, 1 \mathrm{H}, \text { pyrimidine }), 6.926\left(\mathrm{~s}, 2 \mathrm{H},-\mathrm{NH}_{2}\right), 7.126-7.868(\mathrm{~m}, 12 \mathrm{H} \text {, aromatic } \\
\text { proton }), 8.149\left(\mathrm{~s}, 2 \mathrm{H} \text {, phenolic } \mathrm{OH} \text { of } \mathrm{R}_{2} \mathrm{Te}\right), 10.239(\mathrm{~s}, 1 \mathrm{H} \text {, azomethine })\end{array}$ \\
\hline
\end{tabular}
$s=$ singlet,$q=$ quartet,$t=$ triplet, $m=$ multiplet

The characterstic downfield shifting of proton signal in all complexes observed in region $5.67 \delta \mathrm{ppm}$ is due to pyrimidine proton in Schiff base clearly indicate the coordination through pyrimidine nitrogen atom ${ }^{87}$. The signal due to $-\mathrm{NH}$ proton is observed around $6.51 \delta \mathrm{ppm}$ which remain intact with slight variation in complexes is due to the proton bounded to nitrogen experience quadruple effect ${ }^{87}$. Thus salicylidene-trimethoprim act as a tridentate $-\mathrm{N},-\mathrm{N},-\mathrm{O}$ chelating ligand in Sal-TMP.ArTeCl $\mathrm{C}_{2}$ and Sal-TMP. $\mathrm{Ar}_{2} \mathrm{TeCl}$ complexes giving six coordinate tellurium having distorted octahedral geometry in these complexes as predicated from IR studies as well. The proposed structures are as given below (Figure 2). 


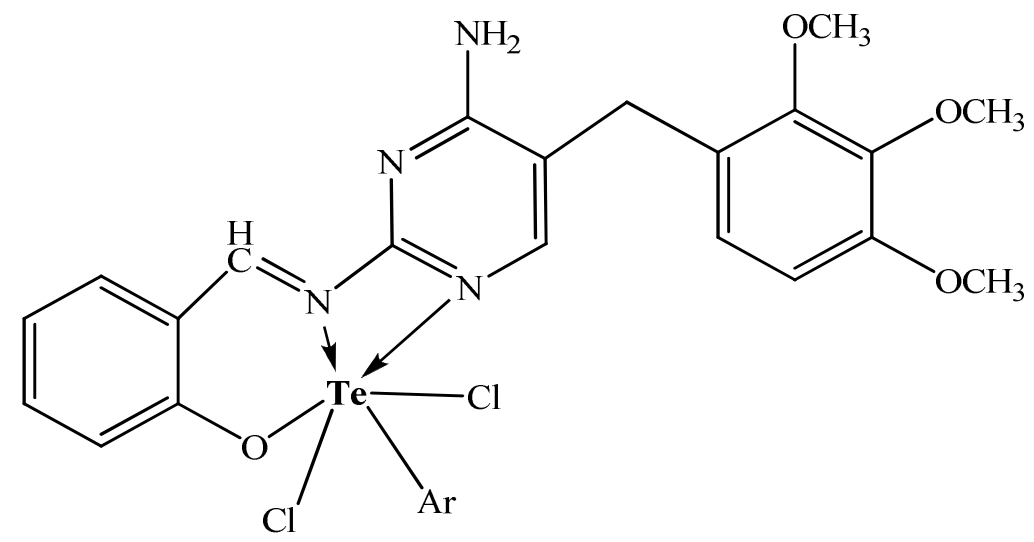<smiles></smiles>

Sal-TMP.Ar ${ }_{2} \mathrm{TeCl}$

$\mathrm{Ar}=p$-methoxyphenyl, $p$-ethoxyphenyl, $p$-hydroxyphenyl and 3-methyl-4-hydroxyphenyl

Figure 2. Proposed structures of complexes

\section{Biological Activity}

The salicylidene-trimethoprim Schiff base (Sal-TMP) and newly synthesized aryltellurium(IV) schiff base complexes were evaluated for their antimicrobial activity in vitro against Gram +ve bacteria (S. aureus ATCC 11632 and B. cereus MTCC 7350), Gram -ve bacteria (E. coli ATCC 35218, P. aeruginosa ATCC 23564, S. typhi ATCC 15499 and $P$. rettgeri DRDE) and fungal strains (A. niger, A. fumigates and A. flavus) by tube dilution method $^{88}$. Dilution of test and standard compounds were prepared double strength nutrient broth- I.P (Antibacterial) and Sabouraud Dextrose Broth -I.P (Antifungal) ${ }^{89}$. The samples were incubated at $37 \pm 1^{\circ} \mathrm{C}$ for $24 \mathrm{~h}$ (bacteria), $25 \pm 1^{\circ} \mathrm{C}$ for 7 days (A. niger), $30 \pm 1^{\circ} \mathrm{C}$ for 15 days (A. flavus), $35 \pm 1{ }^{\circ} \mathrm{C}$ for $72 \mathrm{~h}$ (A. fumigates) respectively and results were recorded in terms of MIC (The lowest concentration of test substances which inhibited values are presented in the Table 4.

The data show that the Sal-TMP complexes of aryltellurium(IV) exhibit more antimicrobial activity towards bacteria as compared to fungii. The antibacterial activity shows following trend. 
Sal-TMP.Ar $\mathrm{ArCl}_{2} \mathrm{Te}$ Sal-TMP.ArTeCl $\mathrm{Cl}_{2} \approx$ Sal-TMP Schiff base

Sal-TMP. $\mathrm{Ar}_{2} \mathrm{TeCl}$ and Sal-TMP.ArTeCl $\mathrm{Cl}_{2}$ complexes have activity towards $S$. typhi and more effectively against $B$. cereus but Schiff base does not show activity against these bacterial strains. Schiff base and its complexes show almost similar activity against fungal strains.

Table 4. Minimum Inhibitory Concentration, MIC, $\mu \mathrm{g} / \mathrm{mL}$; (-) Resistant

\begin{tabular}{|c|c|c|c|c|c|c|c|c|c|}
\hline \multirow[b]{2}{*}{ 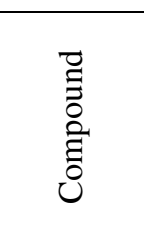 } & \multicolumn{6}{|c|}{ Bacteria strains } & \multicolumn{3}{|c|}{ Fungal strains } \\
\hline & $\begin{array}{c}S . \\
\text { aureus } \\
\text { (ATCC } \\
11632 \text { ) }\end{array}$ & $\begin{array}{l}\text { S. typhi } \\
\text { (ATCC } \\
15499 \text { ) }\end{array}$ & $\begin{array}{c}P . \\
\text { aeruginosa } \\
\text { (ATCC } \\
\text { 23564) }\end{array}$ & $\begin{array}{c}E . \\
a \quad c o l i \\
(\mathrm{ATCC} \\
35218)\end{array}$ & $\begin{array}{c}\text { B. cereus } \\
\text { (MTCC } \\
7350)\end{array}$ & $\begin{array}{c}P . \\
\text { rettgeri } \\
\text { (DRDE } \\
\text { strain) }\end{array}$ & A. niger & $\begin{array}{c}A . \\
\text { fumigates }\end{array}$ & $\begin{array}{l}\text { A. } \\
\text { flavus }\end{array}$ \\
\hline Sal-TMP & 2.5 & - & 1.25 & 5.0 & - & - & 20 & 5.0 & 1.25 \\
\hline I & 2.5 & - & 1.25 & - & 0.625 & 5.0 & - & - & - \\
\hline II & 5.0 & - & 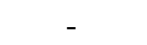 & - & 1.25 & 2.5 & 20 & 5.0 & 1.25 \\
\hline III & - & 20 & 10 & 5.0 & 1.25 & - & 5.0 & - & - \\
\hline IV & 1.25 & 2.5 & 1.25 & 5.0 & - & - & 5.0 & 10 & 5.0 \\
\hline V & - & 20 & 10 & 5.0 & 1.25 & - & 20 & - & - \\
\hline VI & 1.25 & 2.5 & 1.25 & 5.0 & - & - & - & 5.0 & - \\
\hline VII & 1.25 & - & 5.0 & 1.25 & 0.625 & 5.0 & - & 10 & - \\
\hline VIII & 2.5 & - & 1.25 & 5.0 & - & - & 20 & 5.0 & 1.25 \\
\hline
\end{tabular}

\section{Conclusion}

Aryltellurium(IV) and diaryltellurium(IV) dichlorides upon reaction with Schiff base(Sal-TMP) derived from salicylaldehyde and trimethoprim yield new complexes of tellurium(IV). The synthesized complexes were characterized by elemental analyses, conductance measurement, IR and ${ }^{1} \mathrm{H}$ NMR spectral studies. The analytical data suggest that the Sal-TMP Schiff base complexes have 1:1 stoichiometry. The Schiff bases (Sal-TMP) in these complexes functions as a uninegative tridentate ligand through azomethine nitrogen, phenolic oxygen after deprotonation and pyrimidine ring nitrogen atoms. Based on these studies, distorted octahedral geometry with two chelating rings has been assigned to these complexes. The complexes have been observed to possess substantial antimicrobial activity especially against bacteria.

\section{Acknowledgement}

The authors are grateful to M. D. University, Rohtak for providing the necessary facilities. One of the authors (Deepak) is also thankful to UGC New Delhi for providing fellowship. We also thank SAIF, Panjab University Chandigarh for providing the CHN analyses and spectral data.

\section{References}

1. Burchall J J, Corcoran J W and Hahn F E, Eds., Antibiotics, Springer, New York, NY, USA, $1975,3$.

2. Iqbal M S, Khan A H, Loothar B A and Bukhari I H, Med Chem Rev., 2009, 18, 31-42.

3. Blower P J, Аnnu Rep Prog Chem Sect A, 1999, 95, 631-655; DOI:10.1039/A808038K

4. Dhahir S A, Al-Sahib S A, Al Razaq W A and Kadhim N J, Baghdad Sci J., 2010, 7(1), 621.

5. Dr. Karl Thomae Gmb H, (Biberach an der Riss. DE), United States Patent. 4829058.

6. EL-Mossalamy E H, AL-Thabati S A, ALNomaiser F M, Commun Fac Sci Univ Ank Series B, 2005, 51(2), 21-30 
7. Sinha D, Tiwari A K, Singh S, Shukla G, Mishra P, Chandra H and Mishra A K, Eur J Med Chem., 2008, 43(1), 160-165; DOI:10.1016/j.ejmech.2007.03.022

8. Crowe A J, Smith P J and Atassi G, Chem Biol Interact., 1980, 32(1-2), 171-178; DOI:10.1016/0009-2797(80)90075-7

9. Wang M, Wang L F, Li Y Z, Li Q X, Xu Z D and Qu D M, Trans Met Chem., 2001, 26(3), 307-310; DOI:10.1023/A:1007159301849

10. Zishen Wu, Ziqi Gui and Zhenhuan Yen, Synth React Inorg Met Org Chem., 1990, 20(3), 335-344.

11. Desai S B, Desai P B and Desai K R, Hetrocycl Commun., 2001, 7(1), 83-90; DOI:10.1515/HC.2001.7.1.83

12. Dhumwad S D, Gudasi K B and Goudar T R, Indian J Chem., 1994, 33A, 320-324.

13. Przybylski P, Huczynski A, Pyta K, Brzezinski B and Bartl F, Curr Org Chem., 2009, 13(2), 124-148; DOI:10.2174/138527209787193774

14. Pandeya S N, Sriram D, Nath G and De Clercq E, Pharm Acta Hely., 1999, 74(1), 7-11.

15. Karthikeyan M S, Parsad D J, Poojary B, Bhat K S, Holla B S and Kumari N S, Bioorg. Med. Chem., 2006, 14(22), 7482-7489; DOI:10.1016/j.bmc.2006.07.015

16. Holla B S, Akberali P M and Shivananda M K, II Farmaco, 2001, 56(12), 919-927; DOI:10.1016/S0014-827X(01)01124-7

17. Jarrahpour A, Khalili D, De Clercq E, Salmi C and Brunel J M, Molecules, 2007, 12(8), 1720-1730; DOI:10.3390/12081720

18. Da Silva C M, da Silva D L, Modolo L V, Alves R B, de Resende, M A, Martins C V B and de Fatima A J, Adv Res., 2011, 2, 1-8; DOI:10.1016/j.jare.2010.05.004

19. Singh H, Yadav L D S and Mishra S B S, J Inorg Nucl Chem., 1981, 43(7), 17011704; DOI:10.1016/0022-1902(81)80367-3

20. Saravanan G, Pannerselvam P and Prakash C R, J Adv Pharm Techn Res., 2010, 1(3), 320-325; DOI:10.4103/0110-5558.72426

21. Panneerselvam P, Nair R R, Vijayalakshmi G, Subramanian E H and Sridhar S K, Eur. J. Med. Chem. 2005, 40, 225-229; DOI:10.1016/j.ejmech.2004.09.003

22. Singh N K and Singh S B, Indian J Chem., 2001, 40A, 1070-1075.

23. Walsh O M, Meegan M J, Prendergast R M, Nakib T A, Eur J Med Chem., 1996, 31, 989-1000; DOI:10.1016/S0223-5234(97)86178-8

24. Odabasoglu M, Arslan F, Ölmez H and Büyükgüngör O, Dyes and Pigments, 2007, 75(3), 507-515; DOI:10.1016/j.dyepig.2006.06.033

25. Vicini P, Geronikaki A, Incerti M, Busonera B, Poni G, Cabras C A and Colla P L, Bioorg Med Chem., 2003, 11(23), 4785-4789; DOI:10.1016/S09680896(03)00493-0

26. Pandeya S N, Sriram D, Nath G and DeClercq E, Eur J Pharm Sci., 1999, 9, 25-31.

27. Samadhiya S and Halve A, Orient J Chem., 2001, 17, 119-122.

28. Celik Ö, Ulusoy M, Tas E and Ide S, Anal Sciences, 2007, 23, x185.

29. Mahmoud M R, El-Gyar S A, Mousrafa A A and Shaker A, Polyhedron, 1987, 6(5), 1017-1020; DOI:10.1016/S0277-5387(00)80947-X

30. Qing-Yu H, Zheng-Hua M and Ya-Me Z, J Coord Chem., 1990, 21(3), 199-207; DOI:10.1080/00958979009409716

31. Cozzi P G, Chem Soc Rev., 2004, 33, 410-421; DOI:10.1039/B307853C

32. Tarafder M T H, Jin KT, Crouse K A, Ali A M. Yamin, B $M$ and Fun H K, Polyhedron, 2002, 21(25-26), 2547-2554; DOI:10.1016/S0277-5387(02)01188-9

33. Pandeya S N, Sriram D, Clercq E DE., Pannecouque C and Witvrouw M, Indian J Pharm Sci., 1998, 60(4), 207-212. 
34. Fleischman S H and Brooks C L, Proteins: Structure, Function and Genetics, 1990, 7, 52-61.

35. Suling W J, Reynolds R C, Barrow E W, Wilson L N, Piper J R and Barrow W W, J Antimicrobial Chemother., 1998, 42(6), 811-815; DOI:10.1093/jac/42.6.811

36. Gulcan M, Sonmez M and Berber I, Turkey J Chem., 2012, 36(1), 189-200.

37. Raman N, Kulandaisamy A and Jeyasubramanian K, Synth React Inorg Met Org Chem., 2001, 31(7), 1249-1270.

38. Wynne K J and Pearson P S, Inorg Chem., 1971, 10(12), 2735-2739; DOI:10.1021/ic50106a022

39. Wynne K J and Pearson P S, J Chem Soc Commun., 1970, 556-557; DOI:10.1039/C2970000556B

40. Wynne K J, Clark A J and Berg M, J Chem Soc Dalton Trans., 1972, 2370-2374; DOI:10.1039/DT9720002370

41. Clark E R, Collet A J and Naik D G, J Chem Soc Dalton Trans., 1973, 1961-1962; DOI:10.1039/DT9730001961

42. Berg M C, Diss Abstr Int., 1972, 33, 2982.

43. Srivastava T N, Singh M and Singh H B, Indian J Chem., 1982, 21A, 307-309.

44. Srivastava T N, Srivastava R C and Srivastava M, Indian J Chem., 1982, 21A, 539.

45. Srivastava T N, Srivastava R C and Srivastava V K, J Indian Chem Soc., 1983, 60, 891-892.

46. Garad M V, Polyhedron, 1985, 4(8), 1353-1355; DOI:10.1016/S0277-5387(00)86963-6

47. Verma K K and Reena, Synth React Inorg Met-Org Chem., 1999, 29(3), 499-512; DOI:10.1080/00945719909349465

48. Verma K K, Dahiya R and Soni D, Synth React Inorg Met Org Chem., 1999, 29(6), 1033-1052; DOI:10.1080/00945719909349509

49. Verma K K and Dahiya R, Synth React Inorg Met Org Chem., 1999, 29(7), 12991314; DOI:10.1080/00945719909349529

50. Verma K K and Reena, Phosphorus, Sulfur Silicon Related Elements, 1999, 148(1), 227-234; DOI:10.1080/10426509908037013

51. Verma K K and Seema, Int J Chem Sci., 2008, 6, 371-380.

52. Srivastava S, Soni D K and Gupta H S, J Indian Chem Soc., 1996, 73, 255.

53. Narwal J K, Chhabra S, Malik R K, Garg S and Verma K K, Oriental J Chem., 2013, 29, 1339-1349.

54. Chhabra S and Verma K K, J Chem Pharm Res., 2010, 2(4), 569-575.

55. Vogel A I, A Test Book of Organic Chemistry, $3^{\text {rd }}$ Edn., Longman, London, 1975.

56. Weissberger A, Ed., Technique of Organic Chemistry, Vol. 7, $2^{\text {nd }}$ Edn., Interscience Publishers, Inc. N. Y., 1967.

57. Morgan G T and Kellet R E, J Chem Soc., 1926, 1080-1088; DOI:10.1039/JR9262901080

58. Petragnani $\mathrm{N}$ and Stefani $\mathrm{H}$ A, Tellurium in Organic Chemistry, $2^{\text {nd }}$ Edn., Academic Press, London, 2007, 67, 76.

59. Bergman J, Tetrahedron, 1972, 28(12), 3323-3331; DOI:10.1016/S0040-4020(01)93674-9

60. Khandelwal B L, Kumar K and Berry F J, Inorg Chim Acta, 1981, 99(2), 135-137; DOI:10.1016/S0020-1693(00)87958-X

61. Berry F J, Kustan E H, Roshani M and Smith B C, J Organometal Chem., 1975, 99(1), 115-117; DOI:10.1016/S0022-328X(00)86367-6

62. Khandelwal B L, Kumar K and Raina K, Synth React Inorg Met Org Chem., 1981, 11, 65-78.

63. Geary W J, Coord Chem Rev., 1971, 7(1), 81-122; DOI:10.1016/S0010-8545(00)80009-0 
64. Greenwood N N, Straughan B P and Wilson A E, J Chem Soc A, 1968, 2209-2212; DOI: $10.1039 / \mathrm{J} 19680002209$

65. Srivastava K P, Singh A and Singh S K, IOSR J Appl Chem., 2014, 7(4), 16-23; DOI:10.9790/5736-07411623

66. Baker A W and Shulgin A T, J Am Chem Soc., 1959, 81(7), 1523-1529; DOI:10.1021/ja01516a001

67. Freedman H H, J Am Chem Soc., 1961, 83(13), 2900-2905; DOI:10.1021/ja01474a026

68. Flett M St C, Spectrochim Acta, 1957, 10(1), 21-37; DOI:10.1016/0371-1951(57)80160-X

69. Bellamy I J, Infrared Spectra of Complex Molecules, Chapman and Hall Ltd. London, 1975.

70. Matijević-Sosa J, Vinković M and Vikić-Topić D, Croatica Chemica Acta., 2006, 79(3), 489-495.

71. Mishra A P and Soni M, Metal Baesd Drugs, 2008, 71, 243; DOI:10.1155/2008/875410

72. Aminabhavi T M, Biradar N S and Patil C S, Inorganica Chimica Acta., 1983, 78, 107-111; DOI:10.1016/S0020-1693(00)86498-1

73. Biradar N S, Aminabhavi T M and Patil C S, Inorganica Chimica Acta., 1983, 78, 47-50; DOI:10.1016/S0020-1693(00)86487-7

74. Tumer M, Celik C, Koksal H and Serin S, Transition Metal Chem., 1999, 24(5), 525532; DOI:10.1023/A:1006982622965

75. Gwaram N S, Mohd Ali H, Khaledi H, Abdulla M A, Hamid A, Hadi A, Kawi Lin T, Ching C L and Ooi C L, Molecules, 2012, 17(5), 5952;

DOI:10.3390/molecules17055952

76. Rudzinski W E and Aminabhavi T M, Inorganica Chimica Acta., 1982, 67, 177-182; DOI:10.1016/S0020-1693(00)85061-6

77. Osowole A A, Wakil S M and Alao O K, World Appl Sci J., 2015, 33(2), 336-342; DOI:10.5829/idosi.wasj.2015.33.02.22206

78. UR-Rehman S, Faiz A and Nawaz R, J Chil Chem Soc., 2013, 58(1), 1624-1627; DOI:10.4067/S0717-97072013000100026.

79. Mohamed G G and Abd El-Wahab Z H, J Thermal Anal Calorimetry, 2003, 73(1), 347-359; DOI:10.1023/A:1025126801265

80. Verma K K, Soni D and verma, Phosphorus, Sulfur Silicon, 2000, 166(1), 231-241; DOI:10.1080/10426500008076544

81. Pant B C, McWhinnie W R and Dance N S, J Organmetal Chem., 1973, 63, 305-310; DOI:10.1016/S0022-328X(73)80043-9

82. Srivastava T N, Singh J D, Indian J. Chem., 1987, 26A, 260.

83. Chauhan S, Garg S and Verma K K, Chem Sci Trans., 2016, 5(2), 431-441; DOI:10.7598/cst2016.1193

84. Kulkarani Y D, Srivastava S, Abdi S H R and Athar M, Synth React Inorg Met Org Chem., 1985, 15(8), 1043-1059; DOI:10.1080/00945718508060634

85. Chauhan S, Garg S and Verma K K, Res J Pharm Biol Chem Sci., 2016, 7(2), 265-274.

86. Aminabhavi T M and Rudzinski W E, Inorganica Chimica Acta., 1983, 76, L131L134; DOI:10.1016/S0020-1693(00)81478-4

87. Aminabhavi T M and Rudzinski W E, Inorganica Chimica Acta., 1983, 78, 51-55; DOI:10.1016/S0020-1693(00)86488-9

88. Cappuccino J C, Sherman N, Microbiology- A Laboratory Manual, Addison Wesley, California, 1999, 263.

89. Pharmacopoeia of India, Volume 1, Controller of Publications, Ministry of Health Department, Government of India, New Delhi, 2007, 37. 


\title{
Synthesis, Spectral and Biological Studies of Some Salicylidene-Trimethoprim Schiff Base Complexes of Aryltellurium(IV)
}

\author{
DEEPAK, K. K. VERMA and SAPANA GARG* \\ Department of Chemistry, Maharshi Dayanand University, Rohtak-124001, India \\ sapanagarg1511@gmail.com
}

Received 14 February 2017 / Accepted 2 March 2017

\begin{abstract}
A novel monobasic tridentate Schiff base salicylidene-trimethoprim, Sal-TMP, synthesized from trimethoprim and salicylaldehyde, form stable complexes with aryltellurium(IV) trichlorides and diaryltellurium(IV) dichlorides of the type Sal-TMP.ArTeCl ${ }_{2}$ and $\mathrm{Sal}_{-} \mathrm{TMP}_{\mathrm{Ar}} \mathrm{AeCl}$ (where $\mathrm{Ar}=p$-methoxyphenyl, $p$-ethoxyphenyl, $p$-hydroxyphenyl and 3-methyl-4-hydroxyphenyl). These have been characterized by elemental analyses, molar conductance, IR and ${ }^{1} \mathrm{H}$ NMR spectroscopy. The spectral studies predict the bonding of ligand through phenolic oxygen of Schiff base after deprotonation, nitrogen of the azomethine group and pyrimidine nitrogen to give hexacoordinated tellurium(IV) complexes. The complexes have also been screened for their antimicrobial activities against various bacteria and fungi organisms.
\end{abstract}

Keywords: Salicylidene-trimethoprim Schiff base, Aryltellurium(IV), Diaryltellurium(IV), Antibacterial, Antifungal activities

\section{Introduction}

Trimethoprim and its derivatives are broad spectrum antimicrobial agents with anti-parasitic activity $^{1-6}$ and were first described by Roth and coworker ${ }^{5}$. Schiff bases, also known as azomethine due to presence of $-\mathrm{C}=\mathrm{N}$ - group, play important role in biological system, such as anticancer $^{7-12}$, antibacterial ${ }^{13-15}$, antiviral ${ }^{16-18}$, antifungal ${ }^{19-21}$ and other biological properties $^{22-27}$ and also have been extensively used as ligands in coordination chemistry because of their excellent donor abilities as chelating agent ${ }^{28-32}$. Medicinal chemists have reported new derivatives of trimethoprim(TMZ) ${ }^{33-35}$ including the Schiff base derived from salicylaldehyde $2-4,36,37$.

Also, aryltellurium(IV) chlorides are known ${ }^{38-54}$ to act as Lewis acids and form complexes with several N-, O- and S- donor bases. In view of this, we herein report some new complexes derived from aryltellurium(IV) trichlorides, $\mathrm{RTeCl}_{3}$ and diaryltellurium(IV) dichlorides, $\mathrm{R}_{2} \mathrm{TeCl}_{2}$ with salicylidene-trimethoprim Schiff base(Sal-TMP). 


\section{Experimental}

All preparations were carried out under dry $\mathrm{N}_{2}$ atmosphere and the solvents used were purified by standard method ${ }^{55,56}$ before use. The purity of compounds was checked by thin layer chromatography using silica gel-G (Merck). Melting points were determined in open capillary tube and are uncorrected.

Carbon, hydrogen and nitrogen analyses were obtained microanalytically from SAIF, Panjab University Chandigarh on a ThermoFinnigan CHNS analyser. Conductivity was measured in DMSO at $25 \pm 2{ }^{\circ} \mathrm{C}$ with dip type conductivity cell on a microprocessor based conductivity bridge type MICROSIL.

IR $\left(4000-400 \mathrm{~cm}^{-1}\right)$ and far IR $\left(400-50 \mathrm{~cm}^{-1}\right)$ spectra were recorded in $\mathrm{KBr} /$ polyethylene pellets on a FT-Infrared spectrophotometer model RZX (Perkin Elmer) at SAIF, Panjab University Chandigarh. Proton magnetic resonance spectra were recorded in DMSO- $\mathrm{d}_{6}$ using TMS as an internal reference on BRUKER AVANCE II 400 NMR spectrometer. The antimicrobial screening was carried out by tube dilution method at Department of Pharmaceutical Sciences, M. D. University, Rohtak, India.

\section{Preparation of aryltellurium(IV) trichlorides and diaryltellurium(IV) dichlorides}

p-Methoxyphenyltellurium(IV) $\quad$ trichloride $^{57,58}, \quad$ bis(p-methoxyphenyl)tellurium(IV) dichloride ${ }^{58,59}, \quad p$-ethoxyphenyltellurium (IV) trichloride ${ }^{60}$, bis( $p$-ethoxyphenyl)tellurium dichloride $^{60} p$-hydroxyphenyltellurium(IV) trichloride ${ }^{61}$, bis( $p$-hydroxyphenyl) tellurium(IV) dichloride $^{61}$, 3-methyl-4-hydroxyphenyltellurium(IV) trichloride $^{62}$ and bis(3-methyl-4hydroxyphenyl)tellurium(IV) dichloride ${ }^{62}$ were prepared by the reactions of $\mathrm{TeCl}_{4}$ with anisole, phenetole, phenol, $o$-cresol respectively, by the methods reported in the literature ${ }^{57-62}$.

\section{Preparation of salicylidene-trimethoprim Schiff base (Sal-TMP)}

Equimolar quantity of saturated methanolic solution of drug and salicylaldehyde were mixed thoroughly. To this mixture $0.1 \%$ methanolic $\mathrm{KOH}$ was added to adjust the $\mathrm{pH}$ of the solution between 7-8 and was refluxed for 2 hours. A clear yellowish-green coloured solution was obtained. After completion of the reaction, the Schiff base ligand was isolated by crystallization after volume reduction by evaporation. The crystalline product was filtered and dried under vacuum and kept in desiccator over $\mathrm{P}_{4} \mathrm{O}_{10}$ until further use. Yield $=$ $80 \%$, M.pt.(decomp.) $=188-190{ }^{\circ} \mathrm{C}$. Analysis (Calculated) $\mathrm{C}_{21} \mathrm{H}_{22} \mathrm{~N}_{4} \mathrm{O}_{4}: \mathrm{C}(63.95), \mathrm{H}(5.62)$ and $\mathrm{N}(14.20)$; Found: $\mathrm{C}(63.50), \mathrm{H}(5.27)$ and $\mathrm{N}(13.99)$.

\section{Preparation of salicylidene-trimethoprim complexes of aryltellurium(IV) trichlorides} and diaryltellurium(IV) dichlorides

Aryltellurium(IV) trichlorides, $\mathrm{ArTeCl}_{3}$ and diaryltellurium(IV) dichlorides $\mathrm{Ar}_{2} \mathrm{TeCl}_{2}$ (Ar= $p$-methoxyphenyl, $p$-ethoxyphenyl, $p$-hydroxyphenyl and 3-methyl-4-hydroxyphenyl), when reacted with sodium salt of salicylidene-trimethoprim in equimolar ratio, yield SalTMP.ArTeCl $\mathrm{Cl}_{2}$ and Sal-TMP.Ar $\mathrm{TeCl}_{2} \mathrm{Type}$ complexes.

Sodium salt of the ligand was prepared by reacting equimolar (1:1) quantity of sodium metal and Schiff base in methanol. The solvent was distilled off to obtain sodium salt of Schiff base. Then a methanolic saturated solution of $2 \mathrm{mmol}$ of aryltellurium(IV) trichloride or diaryltellurium(IV) dichloride was added dropwise to suspension of $2 \mathrm{mmol}$ of sodium salt of Schiff base in about $50 \mathrm{~mL}$ benzene under reflux. The reaction mixture was further refluxed for 3-4 hours, cooled and precipitated sodium chloride was filtered off. The filtrate was then concentrated to about one third of original volume under reduced pressure and cooled in an ice bath to obtain coloured product. This was filtered, washed with benzene + methanol (1:1) and dried in vacuum desiccator over $\mathrm{P}_{4} \mathrm{O}_{10}$. 


\section{Results and Discussion}

$\mathrm{TeCl}_{4}$ when heated with anisole ${ }^{57-59}$, phenetole ${ }^{60}$, phenol ${ }^{61}, o$-cresol ${ }^{62}$ (Ar-H) appears to undergo Friedel-Crafts type condensation reaction whereby $\mathrm{TeCl}_{3}{ }^{+}$unit attacks a position para to the methoxy/ethoxy/hydroxy groups in the aromatic rings, thus resulting in the formation of aryltellurium(IV) trichlorides and diaryltellurium(IV) dichlorides.

$$
\begin{gathered}
\mathrm{Ar}-\mathrm{H}+\mathrm{TeCl}_{4} \longrightarrow \mathrm{ArTeCl}_{3}+\mathrm{HCl} \\
2 \mathrm{Ar}-\mathrm{H}+\mathrm{TeCl}_{4} \longrightarrow \mathrm{Ar}_{2} \mathrm{TeCl}_{2}+2 \mathrm{HCl}
\end{gathered}
$$

Preparation of salicylidene-trimethoprim Schiff base (Sal-TMP) by the reaction of trimethoprim drug and salicylaldehyde can be represented by following equations.

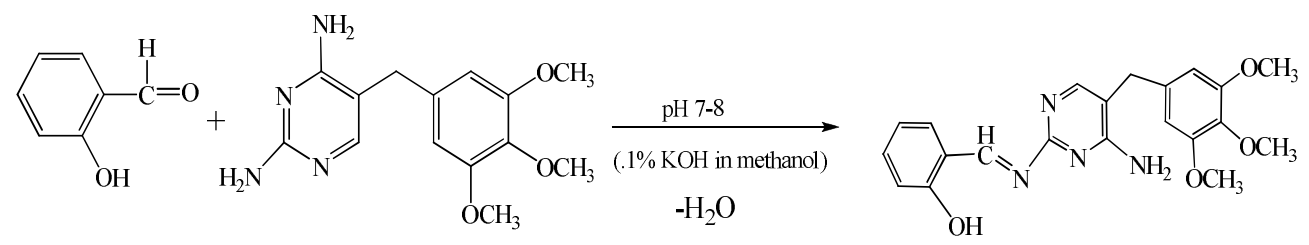

Sodium salt of salicylidene-trimethoprim Schiff base (Sal-TMP) reacts with aryltellurium(IV) trichlorides and diaryltellurium(IV) dichlorides in 1:1 molar ratio to yield the corresponding aryltellurium(IV) complexes.

$$
\begin{aligned}
& \mathrm{Sal}-\mathrm{TMP}+\mathrm{ArTeCl}_{3} \underset{\mathrm{NaCl}}{\stackrel{\mathrm{Na} / \mathrm{CH}_{3} \mathrm{OH}}{\longrightarrow}} \text { (Sal-TMP).ArTeCl } 2 \\
& \mathrm{Sal}-\mathrm{TMP}+\mathrm{Ar}_{2} \mathrm{TeCl}_{2} \underset{-\mathrm{NaCl}}{\stackrel{\mathrm{Na} / \mathrm{CH}_{3} \mathrm{OH}}{\longrightarrow}} \text { (Sal-TMP). } \mathrm{Ar}_{2} \mathrm{TeCl}_{2}
\end{aligned}
$$

All the tellurium(IV) complexes are coloured, crystalline solids, stable at room temperature and non-hygroscopic in nature. The complexes have been analysed for their tellurium, chlorine, carbon, hydrogen and nitrogen contents and the data along with their physical properties and yields are presented in Table 1.

\section{Conductance studies}

Molar conductance $\left(\Lambda_{M}\right)$ data for the complexes in DMSO are complied in Table 1. The $\Lambda_{M}$ value at $c a .10^{-3} \mathrm{M}$ for aryltellurium(IV) complexes in DMSO lie in the range 27.36-91.83 S $\mathrm{cm}^{2} \mathrm{~mol}^{-1}$ which predict the non electrolyte to $1: 1$ electrolyte $^{63,64}$ type behaviour of these complexes in DMSO, probably due to ionization into $\mathrm{ArTeCl}$.Sal- $\mathrm{TMP}^{+} / \mathrm{Ar}_{2} \mathrm{Te}$.Sal-TMP ${ }^{+}$ and $\mathrm{Cl}^{-}$in DMSO. This conductance behavoiur of tellurium(IV) salicylidene-trimethoprim Schiff base complexes is different from those of transition metal complexes ${ }^{65}$, which are reported to be non-electrolytes. The higher $\Lambda_{\mathrm{M}}$ values for some complexes may be due to steric factors and donor behaviour of DMSO to result in probable dissociation into solvated cation and $\mathrm{L}^{-}$along with $\mathrm{Cl}^{-}$in DMSO.

\section{Infrared spectra}

The IR data of Schiff base and its tellurium(IV) complexes are listed in Table 2. The spectra of Sal-TMP Schiff base complexes are quite complex and an attempt has therefore been made to identify the donor sites by comparing the spectra of complexes with parent ligand and $\mathrm{ArTeCl}_{3} / \mathrm{Ar}_{2} \mathrm{TeCl}_{2}$. 
Table 1. Analytical data, molar conductance and physical properties of salicylidene-trimethoprim Schiff base (Sal-TMP) complexes of tellurium(IV)

\begin{tabular}{|c|c|c|c|c|c|c|c|c|c|c|}
\hline \multirow[b]{2}{*}{$\begin{array}{l}\text { Compound } \\
\text { No. }\end{array}$} & \multirow[b]{2}{*}{$\begin{array}{l}\text { Complex } \\
\text { (Ar) }\end{array}$} & \multirow{2}{*}{$\begin{array}{c}\text { Empirical } \\
\text { formula } \\
\text { (Formula Wt.) }\end{array}$} & \multirow[b]{2}{*}{$\begin{array}{l}\text { Colour } \\
\text { (Yield, \%) }\end{array}$} & \multirow{2}{*}{$\begin{array}{l}\text { M. Pt. } \\
{ }^{\circ} \mathrm{C} \\
\text { dec. }\end{array}$} & \multicolumn{5}{|c|}{ Analyses \% Found (Calculated) } & \multirow{2}{*}{$\begin{array}{l}\Lambda_{\mathrm{M}} \text { at } c a \\
10^{-3} \mathrm{M} \mathrm{S} \\
\mathrm{cm}^{2} \mathrm{~mol}^{-1} \\
\text { in DMSO }\end{array}$} \\
\hline & & & & & $\mathrm{C}$ & $\mathrm{H}$ & $\mathrm{N}$ & $\mathrm{Te}$ & $\mathrm{Cl}$ & \\
\hline $\begin{array}{l}\text { Schiff } \\
\text { Base }\end{array}$ & Sal-TMP & $\mathrm{C}_{21} \mathrm{H}_{22} \mathrm{~N}_{4} \mathrm{O}_{4}$ & $\begin{array}{l}\text { Yellowish } \\
\text {-green }(80)\end{array}$ & $188-190$ & $\begin{array}{c}63.50 \\
(63.95)\end{array}$ & $\begin{array}{c}5.27 \\
(5.62)\end{array}$ & $\begin{array}{l}13.99 \\
(14.20)\end{array}$ & - & - & - \\
\hline I & $\begin{array}{l}\text { (Sal-TMP).ArTeCl }{ }_{2} \\
\text { (p-methoxyphenyl) }\end{array}$ & $\begin{array}{c}\mathrm{C}_{28} \mathrm{H}_{28} \mathrm{Cl}_{2} \mathrm{~N}_{4} \mathrm{O}_{5} \mathrm{Te} \\
(699.05)\end{array}$ & $\begin{array}{l}\text { Light cream } \\
(92)\end{array}$ & $230-232$ & $\begin{array}{c}47.88 \\
(48.11)\end{array}$ & $\begin{array}{c}3.99 \\
(4.04)\end{array}$ & $\begin{array}{c}7.75 \\
(8.01)\end{array}$ & $\begin{array}{c}18.07 \\
(18.24)\end{array}$ & $\begin{array}{c}9.89 \\
(10.01)\end{array}$ & 53.19 \\
\hline II & $\begin{array}{c}\text { (Sal-TMP).ArTeCl } \\
\text { (p-ethoxyphenyl) }\end{array}$ & $\begin{array}{c}\mathrm{C}_{29} \mathrm{H}_{30} \mathrm{Cl}_{2} \mathrm{~N}_{4} \mathrm{O}_{5} \mathrm{Te} \\
(713.08)\end{array}$ & $\begin{array}{c}\text { Cream } \\
(85)\end{array}$ & $258-260$ & $\begin{array}{c}48.12 \\
(48.85)\end{array}$ & $\begin{array}{c}3.87 \\
(4.24)\end{array}$ & $\begin{array}{l}7.52 \\
(7.86)\end{array}$ & $\begin{array}{c}17.38 \\
(17.89)\end{array}$ & $\begin{array}{c}9.52 \\
(9.81)\end{array}$ & 52.88 \\
\hline III & $\begin{array}{c}(\mathrm{Sal}-\mathrm{TMP}) \cdot \mathrm{ArTeCl}_{2} \\
(p \text {-hydroxyphenyl })\end{array}$ & $\begin{array}{c}\mathrm{C}_{27} \mathrm{H}_{26} \mathrm{Cl}_{2} \mathrm{~N}_{4} \mathrm{O}_{5} \mathrm{Te} \\
(685.03)\end{array}$ & $\begin{array}{l}\text { Dark cream } \\
\quad(94)\end{array}$ & $208-210$ & $\begin{array}{c}46.78 \\
(47.34)\end{array}$ & $\begin{array}{c}3.50 \\
(3.83)\end{array}$ & $\begin{array}{c}7.88 \\
(8.18)\end{array}$ & $\begin{array}{c}18.28 \\
(18.63)\end{array}$ & $\begin{array}{c}9.98 \\
(10.21)\end{array}$ & 55.73 \\
\hline IV & $\begin{array}{c}\text { (Sal-TMP).ArTeCl } \\
\text { (3-methyl-4- } \\
\text { hydroxyphenyl) }\end{array}$ & $\begin{array}{c}\mathrm{C}_{28} \mathrm{H}_{28} \mathrm{Cl}_{2} \mathrm{~N}_{4} \mathrm{O}_{5} \mathrm{Te} \\
(699.05)\end{array}$ & $\begin{array}{l}\text { Light } \\
\text { cream } \\
(80)\end{array}$ & $198-200$ & $\begin{array}{c}47.58 \\
(48.11)\end{array}$ & $\begin{array}{c}3.84 \\
(4.04)\end{array}$ & $\begin{array}{c}7.80 \\
(8.01)\end{array}$ & $\begin{array}{c}18.15 \\
(18.24)\end{array}$ & $\begin{array}{c}9.85 \\
(10.01)\end{array}$ & 58.68 \\
\hline $\mathrm{V}$ & $\begin{array}{c}(\mathrm{Sal}-\mathrm{TMP}) \cdot \mathrm{Ar}_{2} \mathrm{TeCl} \\
(p \text {-methoxyphenyl })\end{array}$ & $\begin{array}{c}\mathrm{C}_{35} \mathrm{H}_{35} \mathrm{ClN}_{4} \mathrm{O}_{6} \mathrm{Te} \\
(770.73)\end{array}$ & $\begin{array}{c}\text { Pale yellow } \\
\text { (84) }\end{array}$ & $130-132$ & $\begin{array}{c}53.84 \\
(54.54)\end{array}$ & $\begin{array}{c}4.31 \\
(4.58)\end{array}$ & $\begin{array}{c}7.11 \\
(7.27)\end{array}$ & $\begin{array}{c}16.43 \\
(16.56)\end{array}$ & $\begin{array}{c}4.42 \\
(4.60)\end{array}$ & 91.83 \\
\hline VI & $\begin{array}{c}\text { (Sal-TMP).Ar }{ }_{2} \mathrm{TeCl} \\
(p \text {-ethoxyphenyl) }\end{array}$ & $\begin{array}{c}\mathrm{C}_{37} \mathrm{H}_{39} \mathrm{ClN}_{4} \mathrm{O}_{6} \mathrm{Te} \\
(798.78)\end{array}$ & $\begin{array}{l}\text { Light yellow } \\
\text { (86) }\end{array}$ & $150-152$ & $\begin{array}{c}54.84 \\
(55.63)\end{array}$ & $\begin{array}{c}4.53 \\
(4.92)\end{array}$ & $\begin{array}{c}6.84 \\
(7.01)\end{array}$ & $\begin{array}{c}15.50 \\
(15.97)\end{array}$ & $\begin{array}{c}4.30 \\
(4.44)\end{array}$ & 35.90 \\
\hline VII & $\begin{array}{l}\text { (Sal-TMP).Ar } \mathrm{Ar}_{2} \mathrm{TeCl} \\
(p \text {-hydroxyphenyl) }\end{array}$ & $\begin{array}{c}\mathrm{C}_{33} \mathrm{H}_{31} \mathrm{ClN}_{4} \mathrm{O}_{6} \mathrm{Te} \\
(742.68)\end{array}$ & $\begin{array}{l}\text { Red } \\
(89)\end{array}$ & $146-148$ & $\begin{array}{c}52.84 \\
(53.37)\end{array}$ & $\begin{array}{c}4.01 \\
(4.21)\end{array}$ & $\begin{array}{c}7.27 \\
(7.54)\end{array}$ & $\begin{array}{c}16.89 \\
(17.18)\end{array}$ & $\begin{array}{c}4.52 \\
(4.77)\end{array}$ & 36.00 \\
\hline VIII & $\begin{array}{c}\text { (Sal-TMP). } \mathrm{Ar}_{2} \mathrm{TeCl} \\
\text { (3-methyl-4- } \\
\text { hydroxyphenyl) }\end{array}$ & $\begin{array}{c}\mathrm{C}_{35} \mathrm{H}_{35} \mathrm{ClN}_{4} \mathrm{O}_{6} \mathrm{Te} \\
(770.73)\end{array}$ & $\begin{array}{l}\text { Brown } \\
(78)\end{array}$ & $140-142$ & $\begin{array}{l}53.80 \\
(54.54)\end{array}$ & $\begin{array}{c}4.34 \\
(4.58)\end{array}$ & $\begin{array}{c}7.09 \\
(7.27)\end{array}$ & $\begin{array}{c}16.45 \\
(16.56)\end{array}$ & $\begin{array}{c}4.32 \\
(4.60)\end{array}$ & 27.36 \\
\hline
\end{tabular}


Table 2. Important infrared absorption bands $\left(\mathrm{cm}^{-1}\right)$ of Schiff base (Sal-TMP) and complexes

\begin{tabular}{|c|c|c|c|c|c|c|c|}
\hline Compound & $v_{(O-}$ & $v_{(1}$ & $v_{(C}$ & $v_{(C}$ & $v_{(\mathrm{C}-}$ & $v_{(\mathrm{Te}-\mathrm{N})}$ & $v_{(\mathrm{Te}}$ \\
\hline Sal-TMP & $2836 \mathrm{w}$ & $3317 \mathrm{~m} \mathrm{3119} \mathrm{m}$ & $1636 \mathrm{sh}$ & $1633 \mathrm{w} 1593 \mathrm{~s}$ & $1263 \mathrm{~s}$ & - & - \\
\hline $\bar{I}$ & - & $3323 \mathrm{~m} 3184 \mathrm{~m}$ & $1674 \mathrm{mb}$ & $1644 \mathrm{mb} 1587 \mathrm{~s}$ & $1341 \mathrm{~s}$ & $415 \mathrm{~m}$ & $288 \mathrm{w}$ \\
\hline II & - & $3323 \mathrm{~m} 3170 \mathrm{~m}$ & $1647 \mathrm{sh}$ & **1586 s & $1304 \mathrm{~s}$ & $420 \mathrm{~m}$ & $295 w$ \\
\hline III & 3405 m* & $3323 \mathrm{~m} 3150 \mathrm{~m}$ & $1674 \mathrm{mb}$ & $1641 \mathrm{mb} 1586 \mathrm{~s}$ & $1341 \mathrm{~s}$ & $419 \mathrm{~s}$ & $270 \mathrm{w}$ \\
\hline IV & 3398 m* & $3319 \mathrm{~m} 3172 \mathrm{~m}$ & $1652 \mathrm{mb}$ & 1649 & $1333 \mathrm{~s}$ & $450 \mathrm{~s}$ & $277 \mathrm{w}$ \\
\hline V & - & $3325 \mathrm{~m}$ & 164 & $* * 1$ & $1333 \mathrm{~s}$ & $416 \mathrm{~s}$ & $290 \mathrm{w}$ \\
\hline VI & - & $3320 \mathrm{~m} 3158 \mathrm{~m}$ & $1640 \mathrm{sh}$ & $* * 1590 \mathrm{~s}$ & $1331 \mathrm{~s}$ & $410 \mathrm{~m}$ & $273 w$ \\
\hline VII & $3401 \mathrm{w}^{*}$ & $3324 \mathrm{~m} 3168 \mathrm{~m}$ & $1674 \mathrm{mb}$ & $1643 \mathrm{mb} 1584 \mathrm{~s}$ & $1340 \mathrm{~s}$ & $418 \mathrm{~m}$ & $285 \mathrm{w}$ \\
\hline VIII & $3463 \mathrm{w}^{*}$ & $3304 \mathrm{~m} 3106 \mathrm{~m}$ & $1634 \mathrm{sh}$ & $1643 \mathrm{mb} 1584 \mathrm{~s}$ & $1340 \mathrm{~s}$ & $422 \mathrm{~m}$ & $287 \mathrm{w}$ \\
\hline
\end{tabular}

$s=$ sharp, m=medium, mb=medium broad, sh=shoulder, $w=w e a k$, "Due to phenolic $\mathrm{OH}$ of Rte and $\mathrm{R}_{2} \mathrm{Te}$ moieties; ${ }^{* *}$ band not resolved due to overlapping of band $\boldsymbol{v}_{(C=N)}$

Examination of the Schiff base (Sal-TMP) spectrum shows the presence of the hydrogen bonded conjugated- chelate ring system ${ }^{66-68}$ with its centre at $\sim 2832 \mathrm{~cm}^{-1}$. Thus intramolecular $\mathrm{H}$ - bonding is occurring by means of the formation of a quasi six- membered ring involving the $\mathrm{OH}----\mathrm{N}=\mathrm{C}$ bond.<smiles>C1=Cc2ccccc2O[NH+]=1</smiles>

Figure 1. Hydrogen bonding

Thus band disappear on chelation with aryltellurium(IV) chlorides ${ }^{69}$. Hydrogen bond contributes to planarity of the molecule which helps in chelation ${ }^{70}$. Also an intense ligand band at $1263 \mathrm{~cm}^{-1}$ (phenolic -C-O) in free ligand has shifted to higher frequency side in complexes. All these indicate that the hydroxyl group of salicylaldehyde of Schiff base is involved in coordination with tellurium ${ }^{71-73}$.

In addition, the spectra of the Schiff base shown shoulder at $1636 \mathrm{~cm}^{-1}$ with slightly resolved weak band at $1633 \mathrm{~cm}^{-1}$ and sharp band at $1593 \mathrm{~cm}^{-1}$ assigned to $v_{(\mathrm{C}=\mathrm{N})}$ mode for vibration of azomethine group ${ }^{2,4,65,74-76}$ and $v_{(\mathrm{C}=\mathrm{N}) \text { pyrim. For pyrimidine ring }}{ }^{4,77}$. These shift in aryltellurium Schiff base complexes towards higher and lower value ${ }^{74-79}$ reflecting that ligand coordinate through nitrogen atom of azomethine and pyrimidine ring.

The medium intensity band at $3317 \mathrm{~cm}^{-1}$ and $3119 \mathrm{~cm}^{-1}$ due to $v_{(\mathrm{N}-\mathrm{H})}$ asymmetric and symmetric vibrations respectively indicate the non involvement of the nitrogen atom of $\mathrm{NH}_{2}$ group attached to pyrimidine in coordination. The appearance of new weak bands around $270-295 \mathrm{~cm}^{-1}$ due to $v_{(\mathrm{Te}-\mathrm{O})}^{80-83}$ mode and medium to strong band in the range of 410-422 $\mathrm{cm}^{-1}$ due to $v_{(\mathrm{Te}-\mathrm{N})}$ mode ${ }^{84}$ further supports the involvement of phenolic oxygen (after deprotonation), azomethine and pyrimidine nitrogen atoms of Schiff base in the coordination.

Thus, IR data predict the tridentate nature of Sal-TMP involving azomethine nitrogen atom, phenolic oxygen after deprotonation and pyrimidine ring nitrogen giving rise to twosix and four membered chelate rings with the tellurium centre. 


\section{${ }^{1}$ H NMR spectra}

In order to identify the solution structure of Schiff base (Sal-TMP) and its complexes, ${ }^{1} \mathrm{H}$ NMR spectra were recorded in DMSO- $\mathrm{d}_{6}$ and given in Table 3. The proton resonance of the $\mathrm{OH}$ group at $10.92 \delta \mathrm{ppm}^{76,85}$ in Schiff base due to presence of intramolecular hydrogen bonding ${ }^{74}$ disappear on complexation indicating the involvement of phenolic oxygen in the coordination via deprotonation ${ }^{76}$. The azomethine protons which resonate as a singlet at $10.02 \delta \mathrm{ppm}$, the coordination of azomethine nitrogen to tellurium in the complexes are clearly demonstrated by a downfield shift of the peak ${ }^{86}$.

Table 3. ${ }^{1} \mathrm{H}$ NMR spectral data of Schiff base (Sal-TMP) and complexes in DMSO- $\mathrm{d}_{6}$

\begin{tabular}{|c|c|}
\hline Compound & Chemical Shift, $\delta$ ppm \\
\hline Sal-TMP & $\begin{array}{l}2.525(\mathrm{~s}, 2 \mathrm{H}, \text { methylene }), \quad 3.551-3.745\left(\mathrm{~s}, \quad 9 \mathrm{H},-\mathrm{OCH}_{3}\right), \quad 5.672(\mathrm{~s}, 1 \mathrm{H}, \\
\text { pyrimidine }), 6.508\left(\mathrm{~s}, 2 \mathrm{H},-\mathrm{NH}_{2}\right), 7.526-8.169(\mathrm{~m}, 6 \mathrm{H}, \text { aromatic proton), } \\
10.021(\mathrm{~s}, 1 \mathrm{H}, \text { azomethine }), 10.921(\mathrm{~s}, 1 \mathrm{H}, \mathrm{Schiff} \text { base } \mathrm{OH})\end{array}$ \\
\hline I & $\begin{array}{l}2.539(\mathrm{~s}, 2 \mathrm{H}, \text { methylene }), 3.598-3.840\left(\mathrm{~s}, 12 \mathrm{H},-\mathrm{OCH}_{3}\right), 6.574(\mathrm{~s}, 1 \mathrm{H}, \\
\text { pyrimidine }), 6.926\left(\mathrm{~s}, 2 \mathrm{H},-\mathrm{NH}_{2}\right), 7.383-7.522(\mathrm{~m}, 10 \mathrm{H}, \text { aromatic proton }), \\
10.209(\mathrm{~s}, 1 \mathrm{H}, \text { azomethine })\end{array}$ \\
\hline II & $\begin{array}{l}1.335\left(\mathrm{t}, 3 \mathrm{H},-\mathrm{OCH}_{2} \mathrm{CH}_{3}\right), 2.546(\mathrm{~s}, 2 \mathrm{H}, \text { methylene }), 3.963\left(\mathrm{q}, 2 \mathrm{H},-\mathrm{OCH}_{2} \mathrm{CH}_{3}\right) \text {, } \\
3.515-3.875\left(\mathrm{~s}, 9 \mathrm{H},-\mathrm{OCH}_{3}\right), 6.540(\mathrm{~s}, 1 \mathrm{H}, \text { pyrimidine }), 6.950\left(\mathrm{~s}, 2 \mathrm{H},-\mathrm{NH}_{2}\right), \\
6.993-7.809(\mathrm{~m}, 10 \mathrm{H} \text {, aromatic proton), } 10.193(\mathrm{~s}, 1 \mathrm{H} \text {, azomethine })\end{array}$ \\
\hline III & $\begin{array}{l}2.548(\mathrm{~s}, 2 \mathrm{H}, \quad \text { methylene }), \quad 3.601-3.783\left(\mathrm{~s}, 9 \mathrm{H},-\mathrm{OCH}_{3}\right), \quad 6.569(\mathrm{~s}, 1 \mathrm{H}, \\
\text { pyrimidine }), 6.914\left(\mathrm{~s}, 2 \mathrm{H},-\mathrm{NH}_{2}\right), 7.370-7.787(\mathrm{~m}, 10 \mathrm{H}, \text { aromatic proton }), \\
8.155(\mathrm{~s}, 1 \mathrm{H}, \text { phenolic } \mathrm{OH} \text { of } \mathrm{RTe}), 10.201(\mathrm{~s}, 1 \mathrm{H}, \text { azomethine })\end{array}$ \\
\hline IV & $\begin{array}{l}2.536(\mathrm{~s}, 2 \mathrm{H}, \text { methylene }), 2.527\left(\mathrm{~s}, 3 \mathrm{H},-\mathrm{CH}_{3}\right), 3.587-3.770\left(\mathrm{~s}, 9 \mathrm{H},-\mathrm{OCH}_{3}\right) \text {, } \\
\text { 6.605(s, } 1 \mathrm{H}, \text { pyrimidine }), 6.918\left(\mathrm{~s}, 2 \mathrm{H},-\mathrm{NH}_{2}\right), 7.051-7.671(\mathrm{~m}, 9 \mathrm{H} \text {, aromatic } \\
\text { proton }), 8.251(\mathrm{~s}, 1 \mathrm{H} \text {, phenolic } \mathrm{OH} \text { of } \mathrm{RTe}), 10.239(\mathrm{~s}, 1 \mathrm{H} \text {, azomethine })\end{array}$ \\
\hline V & $\begin{array}{l}2.528(\mathrm{~s}, 2 \mathrm{H}, \text { methylene }), 3.673-3.877\left(\mathrm{~s}, 15 \mathrm{H},-\mathrm{OCH}_{3}\right), 6.516(\mathrm{~s}, 1 \mathrm{H} \text {, } \\
\text { pyrimidine }), 6.882\left(\mathrm{~s}, 2 \mathrm{H},-\mathrm{NH}_{2}\right), 6.910-7.804(\mathrm{~m}, 14 \mathrm{H}, \text { aromatic proton }) \\
10.186(\mathrm{~s}, 1 \mathrm{H}, \text { azomethine })\end{array}$ \\
\hline VI & $\begin{array}{l}\left.\text { 1.363(t, } 6 \mathrm{H},-\mathrm{OCH}_{2} \mathbf{C H}_{3}\right), 2.531(\mathrm{~s}, 2 \mathrm{H}, \text { methylene }), 4.019\left(\mathrm{q}, 4 \mathrm{H},-\mathrm{OCH}_{2} \mathrm{CH}_{3}\right) \text {, } \\
3.555-3.765\left(\mathrm{~s}, 9 \mathrm{H},-\mathrm{OCH}_{3}\right), 6.510(\mathrm{~s}, 1 \mathrm{H}, \text { pyrimidine }), 6.924\left(\mathrm{~s}, 2 \mathrm{H},-\mathrm{NH}_{2}\right) \text {, } \\
\text { 7.524-7.805(m, } 14 \mathrm{H} \text {, aromatic proton }), 10.216(\mathrm{~s}, 1 \mathrm{H} \text {, azomethine })\end{array}$ \\
\hline VII & $\begin{array}{l}2.545(\mathrm{~s}, 2 \mathrm{H}, \quad \text { methylene }), 3.568-3.926\left(\mathrm{~s}, 9 \mathrm{H},-\mathrm{OCH}_{3}\right), 6.554(\mathrm{~s}, 1 \mathrm{H} \text {, } \\
\text { pyrimidine }), 6.884\left(\mathrm{~s}, 2 \mathrm{H},-\mathrm{NH}_{2}\right), 7.488-7.678(\mathrm{~m}, 14 \mathrm{H}, \text { aromatic proton), } \\
8.249\left(\mathrm{~s}, 2 \mathrm{H}, \text { phenolic } \mathrm{OH} \text { of } \mathrm{R}_{2} \mathrm{Te}\right), 10.238(\mathrm{~s}, 1 \mathrm{H}, \text { azomethine })\end{array}$ \\
\hline VIII & $\begin{array}{l}2.538(\mathrm{~s}, 2 \mathrm{H}, \text { methylene }), 2.529\left(\mathrm{~s}, 6 \mathrm{H},-\mathrm{CH}_{3}\right), 3.555-3.750\left(\mathrm{~s}, 9 \mathrm{H},-\mathrm{OCH}_{3}\right) \text {, } \\
6.502(\mathrm{~s}, 1 \mathrm{H}, \text { pyrimidine }), 6.926\left(\mathrm{~s}, 2 \mathrm{H},-\mathrm{NH}_{2}\right), 7.126-7.868(\mathrm{~m}, 12 \mathrm{H} \text {, aromatic } \\
\text { proton }), 8.149\left(\mathrm{~s}, 2 \mathrm{H} \text {, phenolic } \mathrm{OH} \text { of } \mathrm{R}_{2} \mathrm{Te}\right), 10.239(\mathrm{~s}, 1 \mathrm{H} \text {, azomethine })\end{array}$ \\
\hline
\end{tabular}
$s=$ singlet,$q=$ quartet,$t=$ triplet, $m=$ multiplet

The characterstic downfield shifting of proton signal in all complexes observed in region $5.67 \delta \mathrm{ppm}$ is due to pyrimidine proton in Schiff base clearly indicate the coordination through pyrimidine nitrogen atom ${ }^{87}$. The signal due to $-\mathrm{NH}$ proton is observed around $6.51 \delta \mathrm{ppm}$ which remain intact with slight variation in complexes is due to the proton bounded to nitrogen experience quadruple effect ${ }^{87}$. Thus salicylidene-trimethoprim act as a tridentate $-\mathrm{N},-\mathrm{N},-\mathrm{O}$ chelating ligand in Sal-TMP.ArTeCl $\mathrm{C}_{2}$ and Sal-TMP. $\mathrm{Ar}_{2} \mathrm{TeCl}$ complexes giving six coordinate tellurium having distorted octahedral geometry in these complexes as predicated from IR studies as well. The proposed structures are as given below (Figure 2). 


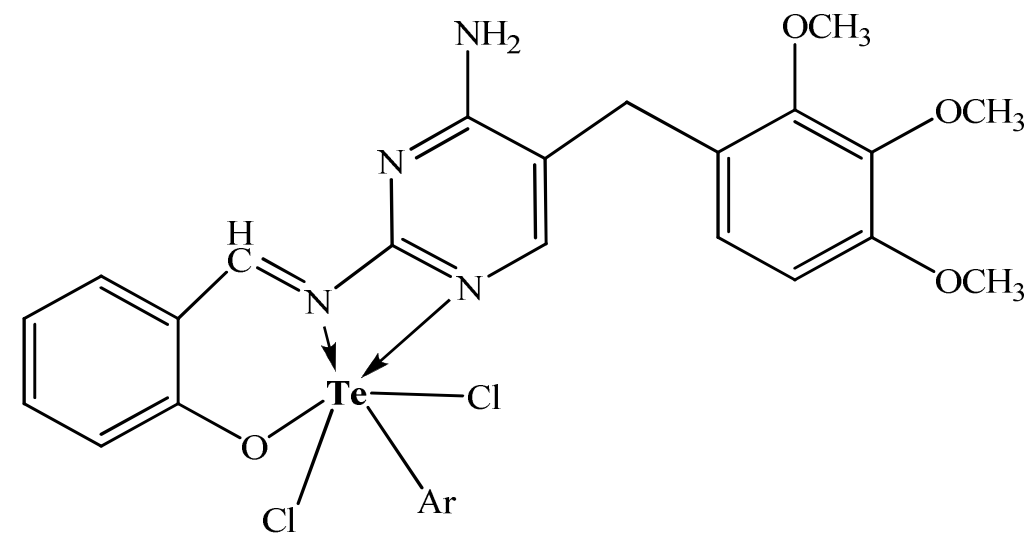<smiles></smiles>

Sal-TMP.Ar ${ }_{2} \mathrm{TeCl}$

$\mathrm{Ar}=p$-methoxyphenyl, $p$-ethoxyphenyl, $p$-hydroxyphenyl and 3-methyl-4-hydroxyphenyl

Figure 2. Proposed structures of complexes

\section{Biological Activity}

The salicylidene-trimethoprim Schiff base (Sal-TMP) and newly synthesized aryltellurium(IV) schiff base complexes were evaluated for their antimicrobial activity in vitro against Gram +ve bacteria (S. aureus ATCC 11632 and B. cereus MTCC 7350), Gram -ve bacteria (E. coli ATCC 35218, P. aeruginosa ATCC 23564, S. typhi ATCC 15499 and $P$. rettgeri DRDE) and fungal strains (A. niger, A. fumigates and A. flavus) by tube dilution method $^{88}$. Dilution of test and standard compounds were prepared double strength nutrient broth- I.P (Antibacterial) and Sabouraud Dextrose Broth -I.P (Antifungal) ${ }^{89}$. The samples were incubated at $37 \pm 1^{\circ} \mathrm{C}$ for $24 \mathrm{~h}$ (bacteria), $25 \pm 1^{\circ} \mathrm{C}$ for 7 days (A. niger), $30 \pm 1^{\circ} \mathrm{C}$ for 15 days (A. flavus), $35 \pm 1{ }^{\circ} \mathrm{C}$ for $72 \mathrm{~h}$ (A. fumigates) respectively and results were recorded in terms of MIC (The lowest concentration of test substances which inhibited values are presented in the Table 4.

The data show that the Sal-TMP complexes of aryltellurium(IV) exhibit more antimicrobial activity towards bacteria as compared to fungii. The antibacterial activity shows following trend. 
Sal-TMP.Ar $\mathrm{ArCl}_{2} \mathrm{Te}$ Sal-TMP.ArTeCl $\mathrm{Cl}_{2} \approx$ Sal-TMP Schiff base

Sal-TMP. $\mathrm{Ar}_{2} \mathrm{TeCl}$ and Sal-TMP.ArTeCl $\mathrm{Cl}_{2}$ complexes have activity towards $S$. typhi and more effectively against $B$. cereus but Schiff base does not show activity against these bacterial strains. Schiff base and its complexes show almost similar activity against fungal strains.

Table 4. Minimum Inhibitory Concentration, MIC, $\mu \mathrm{g} / \mathrm{mL}$; (-) Resistant

\begin{tabular}{|c|c|c|c|c|c|c|c|c|c|}
\hline \multirow[b]{2}{*}{ 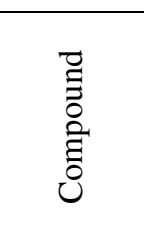 } & \multicolumn{6}{|c|}{ Bacteria strains } & \multicolumn{3}{|c|}{ Fungal strains } \\
\hline & $\begin{array}{c}S . \\
\text { aureus } \\
\text { (ATCC } \\
11632 \text { ) }\end{array}$ & $\begin{array}{l}\text { S. typhi } \\
\text { (ATCC } \\
15499 \text { ) }\end{array}$ & $\begin{array}{c}P . \\
\text { aeruginosa } \\
\text { (ATCC } \\
\text { 23564) }\end{array}$ & $\begin{array}{c}E . \\
a \quad c o l i \\
(\mathrm{ATCC} \\
35218)\end{array}$ & $\begin{array}{c}\text { B. cereus } \\
\text { (MTCC } \\
7350)\end{array}$ & $\begin{array}{c}P . \\
\text { rettgeri } \\
\text { (DRDE } \\
\text { strain) }\end{array}$ & A. niger & $\begin{array}{c}A . \\
\text { fumigates }\end{array}$ & $\begin{array}{l}\text { A. } \\
\text { flavus }\end{array}$ \\
\hline Sal-TMP & 2.5 & - & 1.25 & 5.0 & - & - & 20 & 5.0 & 1.25 \\
\hline I & 2.5 & - & 1.25 & - & 0.625 & 5.0 & - & - & - \\
\hline II & 5.0 & - & 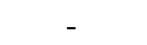 & - & 1.25 & 2.5 & 20 & 5.0 & 1.25 \\
\hline III & - & 20 & 10 & 5.0 & 1.25 & - & 5.0 & - & - \\
\hline IV & 1.25 & 2.5 & 1.25 & 5.0 & - & - & 5.0 & 10 & 5.0 \\
\hline V & - & 20 & 10 & 5.0 & 1.25 & - & 20 & - & - \\
\hline VI & 1.25 & 2.5 & 1.25 & 5.0 & - & - & - & 5.0 & - \\
\hline VII & 1.25 & - & 5.0 & 1.25 & 0.625 & 5.0 & - & 10 & - \\
\hline VIII & 2.5 & - & 1.25 & 5.0 & - & - & 20 & 5.0 & 1.25 \\
\hline
\end{tabular}

\section{Conclusion}

Aryltellurium(IV) and diaryltellurium(IV) dichlorides upon reaction with Schiff base(Sal-TMP) derived from salicylaldehyde and trimethoprim yield new complexes of tellurium(IV). The synthesized complexes were characterized by elemental analyses, conductance measurement, IR and ${ }^{1} \mathrm{H}$ NMR spectral studies. The analytical data suggest that the Sal-TMP Schiff base complexes have 1:1 stoichiometry. The Schiff bases (Sal-TMP) in these complexes functions as a uninegative tridentate ligand through azomethine nitrogen, phenolic oxygen after deprotonation and pyrimidine ring nitrogen atoms. Based on these studies, distorted octahedral geometry with two chelating rings has been assigned to these complexes. The complexes have been observed to possess substantial antimicrobial activity especially against bacteria.

\section{Acknowledgement}

The authors are grateful to M. D. University, Rohtak for providing the necessary facilities. One of the authors (Deepak) is also thankful to UGC New Delhi for providing fellowship. We also thank SAIF, Panjab University Chandigarh for providing the CHN analyses and spectral data.

\section{References}

1. Burchall J J, Corcoran J W and Hahn F E, Eds., Antibiotics, Springer, New York, NY, USA, $1975,3$.

2. Iqbal M S, Khan A H, Loothar B A and Bukhari I H, Med Chem Rev., 2009, 18, 31-42.

3. Blower P J, Аnnu Rep Prog Chem Sect A, 1999, 95, 631-655; DOI:10.1039/A808038K

4. Dhahir S A, Al-Sahib S A, Al Razaq W A and Kadhim N J, Baghdad Sci J., 2010, 7(1), 621.

5. Dr. Karl Thomae Gmb H, (Biberach an der Riss. DE), United States Patent. 4829058.

6. EL-Mossalamy E H, AL-Thabati S A, ALNomaiser F M, Commun Fac Sci Univ Ank Series B, 2005, 51(2), 21-30 
7. Sinha D, Tiwari A K, Singh S, Shukla G, Mishra P, Chandra H and Mishra A K, Eur J Med Chem., 2008, 43(1), 160-165; DOI:10.1016/j.ejmech.2007.03.022

8. Crowe A J, Smith P J and Atassi G, Chem Biol Interact., 1980, 32(1-2), 171-178; DOI:10.1016/0009-2797(80)90075-7

9. Wang M, Wang L F, Li Y Z, Li Q X, Xu Z D and Qu D M, Trans Met Chem., 2001, 26(3), 307-310; DOI:10.1023/A:1007159301849

10. Zishen Wu, Ziqi Gui and Zhenhuan Yen, Synth React Inorg Met Org Chem., 1990, 20(3), 335-344.

11. Desai S B, Desai P B and Desai K R, Hetrocycl Commun., 2001, 7(1), 83-90; DOI:10.1515/HC.2001.7.1.83

12. Dhumwad S D, Gudasi K B and Goudar T R, Indian J Chem., 1994, 33A, 320-324.

13. Przybylski P, Huczynski A, Pyta K, Brzezinski B and Bartl F, Curr Org Chem., 2009, 13(2), 124-148; DOI:10.2174/138527209787193774

14. Pandeya S N, Sriram D, Nath G and De Clercq E, Pharm Acta Hely., 1999, 74(1), 7-11.

15. Karthikeyan M S, Parsad D J, Poojary B, Bhat K S, Holla B S and Kumari N S, Bioorg. Med. Chem., 2006, 14(22), 7482-7489; DOI:10.1016/j.bmc.2006.07.015

16. Holla B S, Akberali P M and Shivananda M K, II Farmaco, 2001, 56(12), 919-927; DOI:10.1016/S0014-827X(01)01124-7

17. Jarrahpour A, Khalili D, De Clercq E, Salmi C and Brunel J M, Molecules, 2007, 12(8), 1720-1730; DOI:10.3390/12081720

18. Da Silva C M, da Silva D L, Modolo L V, Alves R B, de Resende, M A, Martins C V B and de Fatima A J, Adv Res., 2011, 2, 1-8; DOI:10.1016/j.jare.2010.05.004

19. Singh H, Yadav L D S and Mishra S B S, J Inorg Nucl Chem., 1981, 43(7), 17011704; DOI:10.1016/0022-1902(81)80367-3

20. Saravanan G, Pannerselvam P and Prakash C R, J Adv Pharm Techn Res., 2010, 1(3), 320-325; DOI:10.4103/0110-5558.72426

21. Panneerselvam P, Nair R R, Vijayalakshmi G, Subramanian E H and Sridhar S K, Eur. J. Med. Chem. 2005, 40, 225-229; DOI:10.1016/j.ejmech.2004.09.003

22. Singh N K and Singh S B, Indian J Chem., 2001, 40A, 1070-1075.

23. Walsh O M, Meegan M J, Prendergast R M, Nakib T A, Eur J Med Chem., 1996, 31, 989-1000; DOI:10.1016/S0223-5234(97)86178-8

24. Odabasoglu M, Arslan F, Ölmez H and Büyükgüngör O, Dyes and Pigments, 2007, 75(3), 507-515; DOI:10.1016/j.dyepig.2006.06.033

25. Vicini P, Geronikaki A, Incerti M, Busonera B, Poni G, Cabras C A and Colla P L, Bioorg Med Chem., 2003, 11(23), 4785-4789; DOI:10.1016/S09680896(03)00493-0

26. Pandeya S N, Sriram D, Nath G and DeClercq E, Eur J Pharm Sci., 1999, 9, 25-31.

27. Samadhiya S and Halve A, Orient J Chem., 2001, 17, 119-122.

28. Celik Ö, Ulusoy M, Tas E and Ide S, Anal Sciences, 2007, 23, x185.

29. Mahmoud M R, El-Gyar S A, Mousrafa A A and Shaker A, Polyhedron, 1987, 6(5), 1017-1020; DOI:10.1016/S0277-5387(00)80947-X

30. Qing-Yu H, Zheng-Hua M and Ya-Me Z, J Coord Chem., 1990, 21(3), 199-207; DOI:10.1080/00958979009409716

31. Cozzi P G, Chem Soc Rev., 2004, 33, 410-421; DOI:10.1039/B307853C

32. Tarafder M T H, Jin KT, Crouse K A, Ali A M. Yamin, B $M$ and Fun H K, Polyhedron, 2002, 21(25-26), 2547-2554; DOI:10.1016/S0277-5387(02)01188-9

33. Pandeya S N, Sriram D, Clercq E DE., Pannecouque C and Witvrouw M, Indian J Pharm Sci., 1998, 60(4), 207-212. 
34. Fleischman S H and Brooks C L, Proteins: Structure, Function and Genetics, 1990, 7, 52-61.

35. Suling W J, Reynolds R C, Barrow E W, Wilson L N, Piper J R and Barrow W W, J Antimicrobial Chemother., 1998, 42(6), 811-815; DOI:10.1093/jac/42.6.811

36. Gulcan M, Sonmez M and Berber I, Turkey J Chem., 2012, 36(1), 189-200.

37. Raman N, Kulandaisamy A and Jeyasubramanian K, Synth React Inorg Met Org Chem., 2001, 31(7), 1249-1270.

38. Wynne K J and Pearson P S, Inorg Chem., 1971, 10(12), 2735-2739; DOI:10.1021/ic50106a022

39. Wynne K J and Pearson P S, J Chem Soc Commun., 1970, 556-557; DOI:10.1039/C2970000556B

40. Wynne K J, Clark A J and Berg M, J Chem Soc Dalton Trans., 1972, 2370-2374; DOI:10.1039/DT9720002370

41. Clark E R, Collet A J and Naik D G, J Chem Soc Dalton Trans., 1973, 1961-1962; DOI:10.1039/DT9730001961

42. Berg M C, Diss Abstr Int., 1972, 33, 2982.

43. Srivastava T N, Singh M and Singh H B, Indian J Chem., 1982, 21A, 307-309.

44. Srivastava T N, Srivastava R C and Srivastava M, Indian J Chem., 1982, 21A, 539.

45. Srivastava T N, Srivastava R C and Srivastava V K, J Indian Chem Soc., 1983, 60, 891-892.

46. Garad M V, Polyhedron, 1985, 4(8), 1353-1355; DOI:10.1016/S0277-5387(00)86963-6

47. Verma K K and Reena, Synth React Inorg Met-Org Chem., 1999, 29(3), 499-512; DOI:10.1080/00945719909349465

48. Verma K K, Dahiya R and Soni D, Synth React Inorg Met Org Chem., 1999, 29(6), 1033-1052; DOI:10.1080/00945719909349509

49. Verma K K and Dahiya R, Synth React Inorg Met Org Chem., 1999, 29(7), 12991314; DOI:10.1080/00945719909349529

50. Verma K K and Reena, Phosphorus, Sulfur Silicon Related Elements, 1999, 148(1), 227-234; DOI:10.1080/10426509908037013

51. Verma K K and Seema, Int J Chem Sci., 2008, 6, 371-380.

52. Srivastava S, Soni D K and Gupta H S, J Indian Chem Soc., 1996, 73, 255.

53. Narwal J K, Chhabra S, Malik R K, Garg S and Verma K K, Oriental J Chem., 2013, 29, 1339-1349.

54. Chhabra S and Verma K K, J Chem Pharm Res., 2010, 2(4), 569-575.

55. Vogel A I, A Test Book of Organic Chemistry, $3^{\text {rd }}$ Edn., Longman, London, 1975.

56. Weissberger A, Ed., Technique of Organic Chemistry, Vol. 7, $2^{\text {nd }}$ Edn., Interscience Publishers, Inc. N. Y., 1967.

57. Morgan G T and Kellet R E, J Chem Soc., 1926, 1080-1088; DOI:10.1039/JR9262901080

58. Petragnani $\mathrm{N}$ and Stefani $\mathrm{H}$ A, Tellurium in Organic Chemistry, $2^{\text {nd }}$ Edn., Academic Press, London, 2007, 67, 76.

59. Bergman J, Tetrahedron, 1972, 28(12), 3323-3331; DOI:10.1016/S0040-4020(01)93674-9

60. Khandelwal B L, Kumar K and Berry F J, Inorg Chim Acta, 1981, 99(2), 135-137; DOI:10.1016/S0020-1693(00)87958-X

61. Berry F J, Kustan E H, Roshani M and Smith B C, J Organometal Chem., 1975, 99(1), 115-117; DOI:10.1016/S0022-328X(00)86367-6

62. Khandelwal B L, Kumar K and Raina K, Synth React Inorg Met Org Chem., 1981, 11, 65-78.

63. Geary W J, Coord Chem Rev., 1971, 7(1), 81-122; DOI:10.1016/S0010-8545(00)80009-0 
64. Greenwood N N, Straughan B P and Wilson A E, J Chem Soc A, 1968, 2209-2212; DOI: $10.1039 / \mathrm{J} 19680002209$

65. Srivastava K P, Singh A and Singh S K, IOSR J Appl Chem., 2014, 7(4), 16-23; DOI:10.9790/5736-07411623

66. Baker A W and Shulgin A T, J Am Chem Soc., 1959, 81(7), 1523-1529; DOI:10.1021/ja01516a001

67. Freedman H H, J Am Chem Soc., 1961, 83(13), 2900-2905; DOI:10.1021/ja01474a026

68. Flett M St C, Spectrochim Acta, 1957, 10(1), 21-37; DOI:10.1016/0371-1951(57)80160-X

69. Bellamy I J, Infrared Spectra of Complex Molecules, Chapman and Hall Ltd. London, 1975.

70. Matijević-Sosa J, Vinković M and Vikić-Topić D, Croatica Chemica Acta., 2006, 79(3), 489-495.

71. Mishra A P and Soni M, Metal Baesd Drugs, 2008, 71, 243; DOI:10.1155/2008/875410

72. Aminabhavi T M, Biradar N S and Patil C S, Inorganica Chimica Acta., 1983, 78, 107-111; DOI:10.1016/S0020-1693(00)86498-1

73. Biradar N S, Aminabhavi T M and Patil C S, Inorganica Chimica Acta., 1983, 78, 47-50; DOI:10.1016/S0020-1693(00)86487-7

74. Tumer M, Celik C, Koksal H and Serin S, Transition Metal Chem., 1999, 24(5), 525532; DOI:10.1023/A:1006982622965

75. Gwaram N S, Mohd Ali H, Khaledi H, Abdulla M A, Hamid A, Hadi A, Kawi Lin T, Ching C L and Ooi C L, Molecules, 2012, 17(5), 5952;

DOI:10.3390/molecules17055952

76. Rudzinski W E and Aminabhavi T M, Inorganica Chimica Acta., 1982, 67, 177-182; DOI:10.1016/S0020-1693(00)85061-6

77. Osowole A A, Wakil S M and Alao O K, World Appl Sci J., 2015, 33(2), 336-342; DOI:10.5829/idosi.wasj.2015.33.02.22206

78. UR-Rehman S, Faiz A and Nawaz R, J Chil Chem Soc., 2013, 58(1), 1624-1627; DOI:10.4067/S0717-97072013000100026.

79. Mohamed G G and Abd El-Wahab Z H, J Thermal Anal Calorimetry, 2003, 73(1), 347-359; DOI:10.1023/A:1025126801265

80. Verma K K, Soni D and verma, Phosphorus, Sulfur Silicon, 2000, 166(1), 231-241; DOI:10.1080/10426500008076544

81. Pant B C, McWhinnie W R and Dance N S, J Organmetal Chem., 1973, 63, 305-310; DOI:10.1016/S0022-328X(73)80043-9

82. Srivastava T N, Singh J D, Indian J. Chem., 1987, 26A, 260.

83. Chauhan S, Garg S and Verma K K, Chem Sci Trans., 2016, 5(2), 431-441; DOI:10.7598/cst2016.1193

84. Kulkarani Y D, Srivastava S, Abdi S H R and Athar M, Synth React Inorg Met Org Chem., 1985, 15(8), 1043-1059; DOI:10.1080/00945718508060634

85. Chauhan S, Garg S and Verma K K, Res J Pharm Biol Chem Sci., 2016, 7(2), 265-274.

86. Aminabhavi T M and Rudzinski W E, Inorganica Chimica Acta., 1983, 76, L131L134; DOI:10.1016/S0020-1693(00)81478-4

87. Aminabhavi T M and Rudzinski W E, Inorganica Chimica Acta., 1983, 78, 51-55; DOI:10.1016/S0020-1693(00)86488-9

88. Cappuccino J C, Sherman N, Microbiology- A Laboratory Manual, Addison Wesley, California, 1999, 263.

89. Pharmacopoeia of India, Volume 1, Controller of Publications, Ministry of Health Department, Government of India, New Delhi, 2007, 37. 


\title{
Synthesis, Spectral and Biological Studies of Some Salicylidene-Trimethoprim Schiff Base Complexes of Aryltellurium(IV)
}

\author{
DEEPAK, K. K. VERMA and SAPANA GARG* \\ Department of Chemistry, Maharshi Dayanand University, Rohtak-124001, India \\ sapanagarg1511@gmail.com
}

Received 14 February 2017 / Accepted 2 March 2017

\begin{abstract}
A novel monobasic tridentate Schiff base salicylidene-trimethoprim, Sal-TMP, synthesized from trimethoprim and salicylaldehyde, form stable complexes with aryltellurium(IV) trichlorides and diaryltellurium(IV) dichlorides of the type Sal-TMP.ArTeCl ${ }_{2}$ and $\mathrm{Sal}_{-} \mathrm{TMP}_{\mathrm{Ar}} \mathrm{AeCl}$ (where $\mathrm{Ar}=p$-methoxyphenyl, $p$-ethoxyphenyl, $p$-hydroxyphenyl and 3-methyl-4-hydroxyphenyl). These have been characterized by elemental analyses, molar conductance, IR and ${ }^{1} \mathrm{H}$ NMR spectroscopy. The spectral studies predict the bonding of ligand through phenolic oxygen of Schiff base after deprotonation, nitrogen of the azomethine group and pyrimidine nitrogen to give hexacoordinated tellurium(IV) complexes. The complexes have also been screened for their antimicrobial activities against various bacteria and fungi organisms.
\end{abstract}

Keywords: Salicylidene-trimethoprim Schiff base, Aryltellurium(IV), Diaryltellurium(IV), Antibacterial, Antifungal activities

\section{Introduction}

Trimethoprim and its derivatives are broad spectrum antimicrobial agents with anti-parasitic activity $^{1-6}$ and were first described by Roth and coworker ${ }^{5}$. Schiff bases, also known as azomethine due to presence of $-\mathrm{C}=\mathrm{N}$ - group, play important role in biological system, such as anticancer $^{7-12}$, antibacterial ${ }^{13-15}$, antiviral ${ }^{16-18}$, antifungal ${ }^{19-21}$ and other biological properties $^{22-27}$ and also have been extensively used as ligands in coordination chemistry because of their excellent donor abilities as chelating agent ${ }^{28-32}$. Medicinal chemists have reported new derivatives of trimethoprim(TMZ) ${ }^{33-35}$ including the Schiff base derived from salicylaldehyde $2-4,36,37$.

Also, aryltellurium(IV) chlorides are known ${ }^{38-54}$ to act as Lewis acids and form complexes with several N-, O- and S- donor bases. In view of this, we herein report some new complexes derived from aryltellurium(IV) trichlorides, $\mathrm{RTeCl}_{3}$ and diaryltellurium(IV) dichlorides, $\mathrm{R}_{2} \mathrm{TeCl}_{2}$ with salicylidene-trimethoprim Schiff base(Sal-TMP). 


\section{Experimental}

All preparations were carried out under dry $\mathrm{N}_{2}$ atmosphere and the solvents used were purified by standard method ${ }^{55,56}$ before use. The purity of compounds was checked by thin layer chromatography using silica gel-G (Merck). Melting points were determined in open capillary tube and are uncorrected.

Carbon, hydrogen and nitrogen analyses were obtained microanalytically from SAIF, Panjab University Chandigarh on a ThermoFinnigan CHNS analyser. Conductivity was measured in DMSO at $25 \pm 2{ }^{\circ} \mathrm{C}$ with dip type conductivity cell on a microprocessor based conductivity bridge type MICROSIL.

IR $\left(4000-400 \mathrm{~cm}^{-1}\right)$ and far IR $\left(400-50 \mathrm{~cm}^{-1}\right)$ spectra were recorded in $\mathrm{KBr} /$ polyethylene pellets on a FT-Infrared spectrophotometer model RZX (Perkin Elmer) at SAIF, Panjab University Chandigarh. Proton magnetic resonance spectra were recorded in DMSO- $\mathrm{d}_{6}$ using TMS as an internal reference on BRUKER AVANCE II 400 NMR spectrometer. The antimicrobial screening was carried out by tube dilution method at Department of Pharmaceutical Sciences, M. D. University, Rohtak, India.

\section{Preparation of aryltellurium(IV) trichlorides and diaryltellurium(IV) dichlorides}

p-Methoxyphenyltellurium(IV) $\quad$ trichloride $^{57,58}, \quad$ bis(p-methoxyphenyl)tellurium(IV) dichloride ${ }^{58,59}, \quad p$-ethoxyphenyltellurium (IV) trichloride ${ }^{60}$, bis( $p$-ethoxyphenyl)tellurium dichloride $^{60} p$-hydroxyphenyltellurium(IV) trichloride ${ }^{61}$, bis( $p$-hydroxyphenyl) tellurium(IV) dichloride $^{61}$, 3-methyl-4-hydroxyphenyltellurium(IV) trichloride $^{62}$ and bis(3-methyl-4hydroxyphenyl)tellurium(IV) dichloride ${ }^{62}$ were prepared by the reactions of $\mathrm{TeCl}_{4}$ with anisole, phenetole, phenol, $o$-cresol respectively, by the methods reported in the literature ${ }^{57-62}$.

\section{Preparation of salicylidene-trimethoprim Schiff base (Sal-TMP)}

Equimolar quantity of saturated methanolic solution of drug and salicylaldehyde were mixed thoroughly. To this mixture $0.1 \%$ methanolic $\mathrm{KOH}$ was added to adjust the $\mathrm{pH}$ of the solution between 7-8 and was refluxed for 2 hours. A clear yellowish-green coloured solution was obtained. After completion of the reaction, the Schiff base ligand was isolated by crystallization after volume reduction by evaporation. The crystalline product was filtered and dried under vacuum and kept in desiccator over $\mathrm{P}_{4} \mathrm{O}_{10}$ until further use. Yield $=$ $80 \%$, M.pt.(decomp.) $=188-190{ }^{\circ} \mathrm{C}$. Analysis (Calculated) $\mathrm{C}_{21} \mathrm{H}_{22} \mathrm{~N}_{4} \mathrm{O}_{4}: \mathrm{C}(63.95), \mathrm{H}(5.62)$ and $\mathrm{N}(14.20)$; Found: $\mathrm{C}(63.50), \mathrm{H}(5.27)$ and $\mathrm{N}(13.99)$.

\section{Preparation of salicylidene-trimethoprim complexes of aryltellurium(IV) trichlorides} and diaryltellurium(IV) dichlorides

Aryltellurium(IV) trichlorides, $\mathrm{ArTeCl}_{3}$ and diaryltellurium(IV) dichlorides $\mathrm{Ar}_{2} \mathrm{TeCl}_{2}$ (Ar= $p$-methoxyphenyl, $p$-ethoxyphenyl, $p$-hydroxyphenyl and 3-methyl-4-hydroxyphenyl), when reacted with sodium salt of salicylidene-trimethoprim in equimolar ratio, yield SalTMP.ArTeCl $\mathrm{Cl}_{2}$ and Sal-TMP.Ar $\mathrm{TeCl}_{2} \mathrm{Type}$ complexes.

Sodium salt of the ligand was prepared by reacting equimolar (1:1) quantity of sodium metal and Schiff base in methanol. The solvent was distilled off to obtain sodium salt of Schiff base. Then a methanolic saturated solution of $2 \mathrm{mmol}$ of aryltellurium(IV) trichloride or diaryltellurium(IV) dichloride was added dropwise to suspension of $2 \mathrm{mmol}$ of sodium salt of Schiff base in about $50 \mathrm{~mL}$ benzene under reflux. The reaction mixture was further refluxed for 3-4 hours, cooled and precipitated sodium chloride was filtered off. The filtrate was then concentrated to about one third of original volume under reduced pressure and cooled in an ice bath to obtain coloured product. This was filtered, washed with benzene + methanol (1:1) and dried in vacuum desiccator over $\mathrm{P}_{4} \mathrm{O}_{10}$. 


\section{Results and Discussion}

$\mathrm{TeCl}_{4}$ when heated with anisole ${ }^{57-59}$, phenetole ${ }^{60}$, phenol ${ }^{61}, o$-cresol ${ }^{62}$ (Ar-H) appears to undergo Friedel-Crafts type condensation reaction whereby $\mathrm{TeCl}_{3}{ }^{+}$unit attacks a position para to the methoxy/ethoxy/hydroxy groups in the aromatic rings, thus resulting in the formation of aryltellurium(IV) trichlorides and diaryltellurium(IV) dichlorides.

$$
\begin{gathered}
\mathrm{Ar}-\mathrm{H}+\mathrm{TeCl}_{4} \longrightarrow \mathrm{ArTeCl}_{3}+\mathrm{HCl} \\
2 \mathrm{Ar}-\mathrm{H}+\mathrm{TeCl}_{4} \longrightarrow \mathrm{Ar}_{2} \mathrm{TeCl}_{2}+2 \mathrm{HCl}
\end{gathered}
$$

Preparation of salicylidene-trimethoprim Schiff base (Sal-TMP) by the reaction of trimethoprim drug and salicylaldehyde can be represented by following equations.

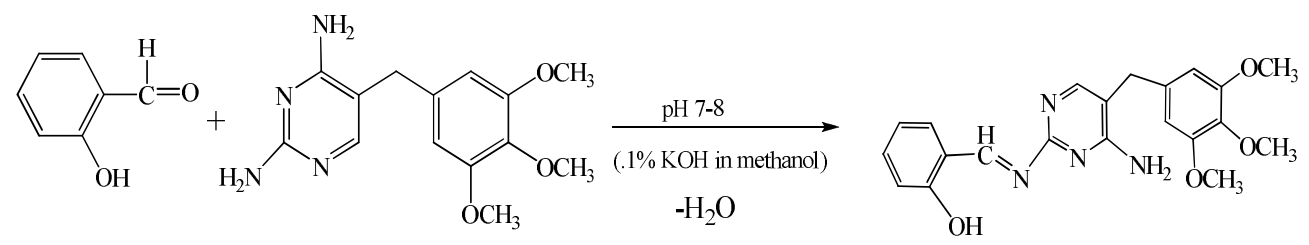

Sodium salt of salicylidene-trimethoprim Schiff base (Sal-TMP) reacts with aryltellurium(IV) trichlorides and diaryltellurium(IV) dichlorides in 1:1 molar ratio to yield the corresponding aryltellurium(IV) complexes.

$$
\begin{aligned}
& \mathrm{Sal}-\mathrm{TMP}+\mathrm{ArTeCl}_{3} \underset{\mathrm{NaCl}}{\stackrel{\mathrm{Na} / \mathrm{CH}_{3} \mathrm{OH}}{\longrightarrow}} \text { (Sal-TMP).ArTeCl } 2 \\
& \mathrm{Sal}-\mathrm{TMP}+\mathrm{Ar}_{2} \mathrm{TeCl}_{2} \underset{-\mathrm{NaCl}}{\stackrel{\mathrm{Na} / \mathrm{CH}_{3} \mathrm{OH}}{\longrightarrow}} \text { (Sal-TMP). } \mathrm{Ar}_{2} \mathrm{TeCl}_{2}
\end{aligned}
$$

All the tellurium(IV) complexes are coloured, crystalline solids, stable at room temperature and non-hygroscopic in nature. The complexes have been analysed for their tellurium, chlorine, carbon, hydrogen and nitrogen contents and the data along with their physical properties and yields are presented in Table 1.

\section{Conductance studies}

Molar conductance $\left(\Lambda_{M}\right)$ data for the complexes in DMSO are complied in Table 1. The $\Lambda_{M}$ value at $c a .10^{-3} \mathrm{M}$ for aryltellurium(IV) complexes in DMSO lie in the range 27.36-91.83 S $\mathrm{cm}^{2} \mathrm{~mol}^{-1}$ which predict the non electrolyte to $1: 1$ electrolyte $^{63,64}$ type behaviour of these complexes in DMSO, probably due to ionization into $\mathrm{ArTeCl}$.Sal- $\mathrm{TMP}^{+} / \mathrm{Ar}_{2} \mathrm{Te}$.Sal-TMP ${ }^{+}$ and $\mathrm{Cl}^{-}$in DMSO. This conductance behavoiur of tellurium(IV) salicylidene-trimethoprim Schiff base complexes is different from those of transition metal complexes ${ }^{65}$, which are reported to be non-electrolytes. The higher $\Lambda_{\mathrm{M}}$ values for some complexes may be due to steric factors and donor behaviour of DMSO to result in probable dissociation into solvated cation and $\mathrm{L}^{-}$along with $\mathrm{Cl}^{-}$in DMSO.

\section{Infrared spectra}

The IR data of Schiff base and its tellurium(IV) complexes are listed in Table 2. The spectra of Sal-TMP Schiff base complexes are quite complex and an attempt has therefore been made to identify the donor sites by comparing the spectra of complexes with parent ligand and $\mathrm{ArTeCl}_{3} / \mathrm{Ar}_{2} \mathrm{TeCl}_{2}$. 
Table 1. Analytical data, molar conductance and physical properties of salicylidene-trimethoprim Schiff base (Sal-TMP) complexes of tellurium(IV)

\begin{tabular}{|c|c|c|c|c|c|c|c|c|c|c|}
\hline \multirow[b]{2}{*}{$\begin{array}{l}\text { Compound } \\
\text { No. }\end{array}$} & \multirow[b]{2}{*}{$\begin{array}{l}\text { Complex } \\
\text { (Ar) }\end{array}$} & \multirow{2}{*}{$\begin{array}{c}\text { Empirical } \\
\text { formula } \\
\text { (Formula Wt.) }\end{array}$} & \multirow[b]{2}{*}{$\begin{array}{l}\text { Colour } \\
\text { (Yield, \%) }\end{array}$} & \multirow{2}{*}{$\begin{array}{l}\text { M. Pt. } \\
{ }^{\circ} \mathrm{C} \\
\text { dec. }\end{array}$} & \multicolumn{5}{|c|}{ Analyses \% Found (Calculated) } & \multirow{2}{*}{$\begin{array}{l}\Lambda_{\mathrm{M}} \text { at } c a \\
10^{-3} \mathrm{M} \mathrm{S} \\
\mathrm{cm}^{2} \mathrm{~mol}^{-1} \\
\text { in DMSO }\end{array}$} \\
\hline & & & & & $\mathrm{C}$ & $\mathrm{H}$ & $\mathrm{N}$ & $\mathrm{Te}$ & $\mathrm{Cl}$ & \\
\hline $\begin{array}{l}\text { Schiff } \\
\text { Base }\end{array}$ & Sal-TMP & $\mathrm{C}_{21} \mathrm{H}_{22} \mathrm{~N}_{4} \mathrm{O}_{4}$ & $\begin{array}{l}\text { Yellowish } \\
\text {-green }(80)\end{array}$ & $188-190$ & $\begin{array}{c}63.50 \\
(63.95)\end{array}$ & $\begin{array}{c}5.27 \\
(5.62)\end{array}$ & $\begin{array}{l}13.99 \\
(14.20)\end{array}$ & - & - & - \\
\hline I & $\begin{array}{l}\text { (Sal-TMP).ArTeCl }{ }_{2} \\
\text { (p-methoxyphenyl) }\end{array}$ & $\begin{array}{c}\mathrm{C}_{28} \mathrm{H}_{28} \mathrm{Cl}_{2} \mathrm{~N}_{4} \mathrm{O}_{5} \mathrm{Te} \\
(699.05)\end{array}$ & $\begin{array}{l}\text { Light cream } \\
(92)\end{array}$ & $230-232$ & $\begin{array}{c}47.88 \\
(48.11)\end{array}$ & $\begin{array}{c}3.99 \\
(4.04)\end{array}$ & $\begin{array}{c}7.75 \\
(8.01)\end{array}$ & $\begin{array}{c}18.07 \\
(18.24)\end{array}$ & $\begin{array}{c}9.89 \\
(10.01)\end{array}$ & 53.19 \\
\hline II & $\begin{array}{c}\text { (Sal-TMP).ArTeCl } \\
\text { (p-ethoxyphenyl) }\end{array}$ & $\begin{array}{c}\mathrm{C}_{29} \mathrm{H}_{30} \mathrm{Cl}_{2} \mathrm{~N}_{4} \mathrm{O}_{5} \mathrm{Te} \\
(713.08)\end{array}$ & $\begin{array}{c}\text { Cream } \\
(85)\end{array}$ & $258-260$ & $\begin{array}{c}48.12 \\
(48.85)\end{array}$ & $\begin{array}{c}3.87 \\
(4.24)\end{array}$ & $\begin{array}{l}7.52 \\
(7.86)\end{array}$ & $\begin{array}{c}17.38 \\
(17.89)\end{array}$ & $\begin{array}{c}9.52 \\
(9.81)\end{array}$ & 52.88 \\
\hline III & $\begin{array}{c}(\mathrm{Sal}-\mathrm{TMP}) \cdot \mathrm{ArTeCl}_{2} \\
(p \text {-hydroxyphenyl })\end{array}$ & $\begin{array}{c}\mathrm{C}_{27} \mathrm{H}_{26} \mathrm{Cl}_{2} \mathrm{~N}_{4} \mathrm{O}_{5} \mathrm{Te} \\
(685.03)\end{array}$ & $\begin{array}{l}\text { Dark cream } \\
\quad(94)\end{array}$ & $208-210$ & $\begin{array}{c}46.78 \\
(47.34)\end{array}$ & $\begin{array}{c}3.50 \\
(3.83)\end{array}$ & $\begin{array}{c}7.88 \\
(8.18)\end{array}$ & $\begin{array}{c}18.28 \\
(18.63)\end{array}$ & $\begin{array}{c}9.98 \\
(10.21)\end{array}$ & 55.73 \\
\hline IV & $\begin{array}{c}\text { (Sal-TMP).ArTeCl } \\
\text { (3-methyl-4- } \\
\text { hydroxyphenyl) }\end{array}$ & $\begin{array}{c}\mathrm{C}_{28} \mathrm{H}_{28} \mathrm{Cl}_{2} \mathrm{~N}_{4} \mathrm{O}_{5} \mathrm{Te} \\
(699.05)\end{array}$ & $\begin{array}{l}\text { Light } \\
\text { cream } \\
(80)\end{array}$ & $198-200$ & $\begin{array}{c}47.58 \\
(48.11)\end{array}$ & $\begin{array}{c}3.84 \\
(4.04)\end{array}$ & $\begin{array}{c}7.80 \\
(8.01)\end{array}$ & $\begin{array}{c}18.15 \\
(18.24)\end{array}$ & $\begin{array}{c}9.85 \\
(10.01)\end{array}$ & 58.68 \\
\hline $\mathrm{V}$ & $\begin{array}{c}(\mathrm{Sal}-\mathrm{TMP}) \cdot \mathrm{Ar}_{2} \mathrm{TeCl} \\
(p \text {-methoxyphenyl })\end{array}$ & $\begin{array}{c}\mathrm{C}_{35} \mathrm{H}_{35} \mathrm{ClN}_{4} \mathrm{O}_{6} \mathrm{Te} \\
(770.73)\end{array}$ & $\begin{array}{c}\text { Pale yellow } \\
\text { (84) }\end{array}$ & $130-132$ & $\begin{array}{c}53.84 \\
(54.54)\end{array}$ & $\begin{array}{c}4.31 \\
(4.58)\end{array}$ & $\begin{array}{c}7.11 \\
(7.27)\end{array}$ & $\begin{array}{c}16.43 \\
(16.56)\end{array}$ & $\begin{array}{c}4.42 \\
(4.60)\end{array}$ & 91.83 \\
\hline VI & $\begin{array}{c}\text { (Sal-TMP).Ar }{ }_{2} \mathrm{TeCl} \\
(p \text {-ethoxyphenyl) }\end{array}$ & $\begin{array}{c}\mathrm{C}_{37} \mathrm{H}_{39} \mathrm{ClN}_{4} \mathrm{O}_{6} \mathrm{Te} \\
(798.78)\end{array}$ & $\begin{array}{l}\text { Light yellow } \\
\text { (86) }\end{array}$ & $150-152$ & $\begin{array}{c}54.84 \\
(55.63)\end{array}$ & $\begin{array}{c}4.53 \\
(4.92)\end{array}$ & $\begin{array}{c}6.84 \\
(7.01)\end{array}$ & $\begin{array}{c}15.50 \\
(15.97)\end{array}$ & $\begin{array}{c}4.30 \\
(4.44)\end{array}$ & 35.90 \\
\hline VII & $\begin{array}{l}\text { (Sal-TMP).Ar } \mathrm{Ar}_{2} \mathrm{TeCl} \\
(p \text {-hydroxyphenyl) }\end{array}$ & $\begin{array}{c}\mathrm{C}_{33} \mathrm{H}_{31} \mathrm{ClN}_{4} \mathrm{O}_{6} \mathrm{Te} \\
(742.68)\end{array}$ & $\begin{array}{l}\text { Red } \\
(89)\end{array}$ & $146-148$ & $\begin{array}{c}52.84 \\
(53.37)\end{array}$ & $\begin{array}{c}4.01 \\
(4.21)\end{array}$ & $\begin{array}{c}7.27 \\
(7.54)\end{array}$ & $\begin{array}{c}16.89 \\
(17.18)\end{array}$ & $\begin{array}{c}4.52 \\
(4.77)\end{array}$ & 36.00 \\
\hline VIII & $\begin{array}{c}\text { (Sal-TMP). } \mathrm{Ar}_{2} \mathrm{TeCl} \\
\text { (3-methyl-4- } \\
\text { hydroxyphenyl) }\end{array}$ & $\begin{array}{c}\mathrm{C}_{35} \mathrm{H}_{35} \mathrm{ClN}_{4} \mathrm{O}_{6} \mathrm{Te} \\
(770.73)\end{array}$ & $\begin{array}{l}\text { Brown } \\
(78)\end{array}$ & $140-142$ & $\begin{array}{l}53.80 \\
(54.54)\end{array}$ & $\begin{array}{c}4.34 \\
(4.58)\end{array}$ & $\begin{array}{c}7.09 \\
(7.27)\end{array}$ & $\begin{array}{c}16.45 \\
(16.56)\end{array}$ & $\begin{array}{c}4.32 \\
(4.60)\end{array}$ & 27.36 \\
\hline
\end{tabular}


Table 2. Important infrared absorption bands $\left(\mathrm{cm}^{-1}\right)$ of Schiff base (Sal-TMP) and complexes

\begin{tabular}{|c|c|c|c|c|c|c|c|}
\hline Compound & $v_{(O-}$ & $v_{(1}$ & $v_{(C}$ & $v_{(C}$ & $v_{(\mathrm{C}-}$ & $v_{(\mathrm{Te}-\mathrm{N})}$ & $v_{(\mathrm{Te}}$ \\
\hline Sal-TMP & $2836 \mathrm{w}$ & $3317 \mathrm{~m} \mathrm{3119} \mathrm{m}$ & $1636 \mathrm{sh}$ & $1633 \mathrm{w} 1593 \mathrm{~s}$ & $1263 \mathrm{~s}$ & - & - \\
\hline $\bar{I}$ & - & $3323 \mathrm{~m} 3184 \mathrm{~m}$ & $1674 \mathrm{mb}$ & $1644 \mathrm{mb} 1587 \mathrm{~s}$ & $1341 \mathrm{~s}$ & $415 \mathrm{~m}$ & $288 \mathrm{w}$ \\
\hline II & - & $3323 \mathrm{~m} 3170 \mathrm{~m}$ & $1647 \mathrm{sh}$ & **1586 s & $1304 \mathrm{~s}$ & $420 \mathrm{~m}$ & $295 w$ \\
\hline III & 3405 m* & $3323 \mathrm{~m} 3150 \mathrm{~m}$ & $1674 \mathrm{mb}$ & $1641 \mathrm{mb} 1586 \mathrm{~s}$ & $1341 \mathrm{~s}$ & $419 \mathrm{~s}$ & $270 \mathrm{w}$ \\
\hline IV & 3398 m* & $3319 \mathrm{~m} 3172 \mathrm{~m}$ & $1652 \mathrm{mb}$ & 1649 & $1333 \mathrm{~s}$ & $450 \mathrm{~s}$ & $277 \mathrm{w}$ \\
\hline V & - & $3325 \mathrm{~m}$ & 164 & $* * 1$ & $1333 \mathrm{~s}$ & $416 \mathrm{~s}$ & $290 \mathrm{w}$ \\
\hline VI & - & $3320 \mathrm{~m} 3158 \mathrm{~m}$ & $1640 \mathrm{sh}$ & $* * 1590 \mathrm{~s}$ & $1331 \mathrm{~s}$ & $410 \mathrm{~m}$ & $273 w$ \\
\hline VII & $3401 \mathrm{w}^{*}$ & $3324 \mathrm{~m} 3168 \mathrm{~m}$ & $1674 \mathrm{mb}$ & $1643 \mathrm{mb} 1584 \mathrm{~s}$ & $1340 \mathrm{~s}$ & $418 \mathrm{~m}$ & $285 \mathrm{w}$ \\
\hline VIII & $3463 \mathrm{w}^{*}$ & $3304 \mathrm{~m} 3106 \mathrm{~m}$ & $1634 \mathrm{sh}$ & $1643 \mathrm{mb} 1584 \mathrm{~s}$ & $1340 \mathrm{~s}$ & $422 \mathrm{~m}$ & $287 \mathrm{w}$ \\
\hline
\end{tabular}

$s=$ sharp, m=medium, mb=medium broad, sh=shoulder, $w=w e a k$, "Due to phenolic $\mathrm{OH}$ of Rte and $\mathrm{R}_{2} \mathrm{Te}$ moieties; ${ }^{* *}$ band not resolved due to overlapping of band $\boldsymbol{v}_{(C=N)}$

Examination of the Schiff base (Sal-TMP) spectrum shows the presence of the hydrogen bonded conjugated- chelate ring system ${ }^{66-68}$ with its centre at $\sim 2832 \mathrm{~cm}^{-1}$. Thus intramolecular $\mathrm{H}$ - bonding is occurring by means of the formation of a quasi six- membered ring involving the $\mathrm{OH}----\mathrm{N}=\mathrm{C}$ bond.<smiles>C1=Cc2ccccc2O[NH+]=1</smiles>

Figure 1. Hydrogen bonding

Thus band disappear on chelation with aryltellurium(IV) chlorides ${ }^{69}$. Hydrogen bond contributes to planarity of the molecule which helps in chelation ${ }^{70}$. Also an intense ligand band at $1263 \mathrm{~cm}^{-1}$ (phenolic -C-O) in free ligand has shifted to higher frequency side in complexes. All these indicate that the hydroxyl group of salicylaldehyde of Schiff base is involved in coordination with tellurium ${ }^{71-73}$.

In addition, the spectra of the Schiff base shown shoulder at $1636 \mathrm{~cm}^{-1}$ with slightly resolved weak band at $1633 \mathrm{~cm}^{-1}$ and sharp band at $1593 \mathrm{~cm}^{-1}$ assigned to $v_{(\mathrm{C}=\mathrm{N})}$ mode for vibration of azomethine group ${ }^{2,4,65,74-76}$ and $v_{(\mathrm{C}=\mathrm{N}) \text { pyrim. For pyrimidine ring }}{ }^{4,77}$. These shift in aryltellurium Schiff base complexes towards higher and lower value ${ }^{74-79}$ reflecting that ligand coordinate through nitrogen atom of azomethine and pyrimidine ring.

The medium intensity band at $3317 \mathrm{~cm}^{-1}$ and $3119 \mathrm{~cm}^{-1}$ due to $v_{(\mathrm{N}-\mathrm{H})}$ asymmetric and symmetric vibrations respectively indicate the non involvement of the nitrogen atom of $\mathrm{NH}_{2}$ group attached to pyrimidine in coordination. The appearance of new weak bands around $270-295 \mathrm{~cm}^{-1}$ due to $v_{(\mathrm{Te}-\mathrm{O})}^{80-83}$ mode and medium to strong band in the range of 410-422 $\mathrm{cm}^{-1}$ due to $v_{(\mathrm{Te}-\mathrm{N})}$ mode ${ }^{84}$ further supports the involvement of phenolic oxygen (after deprotonation), azomethine and pyrimidine nitrogen atoms of Schiff base in the coordination.

Thus, IR data predict the tridentate nature of Sal-TMP involving azomethine nitrogen atom, phenolic oxygen after deprotonation and pyrimidine ring nitrogen giving rise to twosix and four membered chelate rings with the tellurium centre. 


\section{${ }^{1}$ H NMR spectra}

In order to identify the solution structure of Schiff base (Sal-TMP) and its complexes, ${ }^{1} \mathrm{H}$ NMR spectra were recorded in DMSO- $\mathrm{d}_{6}$ and given in Table 3. The proton resonance of the $\mathrm{OH}$ group at $10.92 \delta \mathrm{ppm}^{76,85}$ in Schiff base due to presence of intramolecular hydrogen bonding ${ }^{74}$ disappear on complexation indicating the involvement of phenolic oxygen in the coordination via deprotonation ${ }^{76}$. The azomethine protons which resonate as a singlet at $10.02 \delta \mathrm{ppm}$, the coordination of azomethine nitrogen to tellurium in the complexes are clearly demonstrated by a downfield shift of the peak ${ }^{86}$.

Table 3. ${ }^{1} \mathrm{H}$ NMR spectral data of Schiff base (Sal-TMP) and complexes in DMSO- $\mathrm{d}_{6}$

\begin{tabular}{|c|c|}
\hline Compound & Chemical Shift, $\delta$ ppm \\
\hline Sal-TMP & $\begin{array}{l}2.525(\mathrm{~s}, 2 \mathrm{H}, \text { methylene }), \quad 3.551-3.745\left(\mathrm{~s}, \quad 9 \mathrm{H},-\mathrm{OCH}_{3}\right), \quad 5.672(\mathrm{~s}, 1 \mathrm{H}, \\
\text { pyrimidine }), 6.508\left(\mathrm{~s}, 2 \mathrm{H},-\mathrm{NH}_{2}\right), 7.526-8.169(\mathrm{~m}, 6 \mathrm{H}, \text { aromatic proton), } \\
10.021(\mathrm{~s}, 1 \mathrm{H}, \text { azomethine }), 10.921(\mathrm{~s}, 1 \mathrm{H}, \mathrm{Schiff} \text { base } \mathrm{OH})\end{array}$ \\
\hline I & $\begin{array}{l}2.539(\mathrm{~s}, 2 \mathrm{H}, \text { methylene }), 3.598-3.840\left(\mathrm{~s}, 12 \mathrm{H},-\mathrm{OCH}_{3}\right), 6.574(\mathrm{~s}, 1 \mathrm{H}, \\
\text { pyrimidine }), 6.926\left(\mathrm{~s}, 2 \mathrm{H},-\mathrm{NH}_{2}\right), 7.383-7.522(\mathrm{~m}, 10 \mathrm{H}, \text { aromatic proton }), \\
10.209(\mathrm{~s}, 1 \mathrm{H}, \text { azomethine })\end{array}$ \\
\hline II & $\begin{array}{l}1.335\left(\mathrm{t}, 3 \mathrm{H},-\mathrm{OCH}_{2} \mathrm{CH}_{3}\right), 2.546(\mathrm{~s}, 2 \mathrm{H}, \text { methylene }), 3.963\left(\mathrm{q}, 2 \mathrm{H},-\mathrm{OCH}_{2} \mathrm{CH}_{3}\right) \text {, } \\
3.515-3.875\left(\mathrm{~s}, 9 \mathrm{H},-\mathrm{OCH}_{3}\right), 6.540(\mathrm{~s}, 1 \mathrm{H}, \text { pyrimidine }), 6.950\left(\mathrm{~s}, 2 \mathrm{H},-\mathrm{NH}_{2}\right), \\
6.993-7.809(\mathrm{~m}, 10 \mathrm{H} \text {, aromatic proton), } 10.193(\mathrm{~s}, 1 \mathrm{H} \text {, azomethine })\end{array}$ \\
\hline III & $\begin{array}{l}2.548(\mathrm{~s}, 2 \mathrm{H}, \quad \text { methylene }), \quad 3.601-3.783\left(\mathrm{~s}, 9 \mathrm{H},-\mathrm{OCH}_{3}\right), \quad 6.569(\mathrm{~s}, 1 \mathrm{H}, \\
\text { pyrimidine }), 6.914\left(\mathrm{~s}, 2 \mathrm{H},-\mathrm{NH}_{2}\right), 7.370-7.787(\mathrm{~m}, 10 \mathrm{H}, \text { aromatic proton }), \\
8.155(\mathrm{~s}, 1 \mathrm{H}, \text { phenolic } \mathrm{OH} \text { of } \mathrm{RTe}), 10.201(\mathrm{~s}, 1 \mathrm{H}, \text { azomethine })\end{array}$ \\
\hline IV & $\begin{array}{l}2.536(\mathrm{~s}, 2 \mathrm{H}, \text { methylene }), 2.527\left(\mathrm{~s}, 3 \mathrm{H},-\mathrm{CH}_{3}\right), 3.587-3.770\left(\mathrm{~s}, 9 \mathrm{H},-\mathrm{OCH}_{3}\right) \text {, } \\
\text { 6.605(s, } 1 \mathrm{H}, \text { pyrimidine }), 6.918\left(\mathrm{~s}, 2 \mathrm{H},-\mathrm{NH}_{2}\right), 7.051-7.671(\mathrm{~m}, 9 \mathrm{H} \text {, aromatic } \\
\text { proton }), 8.251(\mathrm{~s}, 1 \mathrm{H} \text {, phenolic } \mathrm{OH} \text { of } \mathrm{RTe}), 10.239(\mathrm{~s}, 1 \mathrm{H} \text {, azomethine })\end{array}$ \\
\hline V & $\begin{array}{l}2.528(\mathrm{~s}, 2 \mathrm{H}, \text { methylene }), 3.673-3.877\left(\mathrm{~s}, 15 \mathrm{H},-\mathrm{OCH}_{3}\right), 6.516(\mathrm{~s}, 1 \mathrm{H} \text {, } \\
\text { pyrimidine }), 6.882\left(\mathrm{~s}, 2 \mathrm{H},-\mathrm{NH}_{2}\right), 6.910-7.804(\mathrm{~m}, 14 \mathrm{H}, \text { aromatic proton }) \\
10.186(\mathrm{~s}, 1 \mathrm{H}, \text { azomethine })\end{array}$ \\
\hline VI & $\begin{array}{l}\left.\text { 1.363(t, } 6 \mathrm{H},-\mathrm{OCH}_{2} \mathbf{C H}_{3}\right), 2.531(\mathrm{~s}, 2 \mathrm{H}, \text { methylene }), 4.019\left(\mathrm{q}, 4 \mathrm{H},-\mathrm{OCH}_{2} \mathrm{CH}_{3}\right) \text {, } \\
3.555-3.765\left(\mathrm{~s}, 9 \mathrm{H},-\mathrm{OCH}_{3}\right), 6.510(\mathrm{~s}, 1 \mathrm{H}, \text { pyrimidine }), 6.924\left(\mathrm{~s}, 2 \mathrm{H},-\mathrm{NH}_{2}\right) \text {, } \\
\text { 7.524-7.805(m, } 14 \mathrm{H} \text {, aromatic proton }), 10.216(\mathrm{~s}, 1 \mathrm{H} \text {, azomethine })\end{array}$ \\
\hline VII & $\begin{array}{l}2.545(\mathrm{~s}, 2 \mathrm{H}, \quad \text { methylene }), 3.568-3.926\left(\mathrm{~s}, 9 \mathrm{H},-\mathrm{OCH}_{3}\right), 6.554(\mathrm{~s}, 1 \mathrm{H} \text {, } \\
\text { pyrimidine }), 6.884\left(\mathrm{~s}, 2 \mathrm{H},-\mathrm{NH}_{2}\right), 7.488-7.678(\mathrm{~m}, 14 \mathrm{H}, \text { aromatic proton), } \\
8.249\left(\mathrm{~s}, 2 \mathrm{H}, \text { phenolic } \mathrm{OH} \text { of } \mathrm{R}_{2} \mathrm{Te}\right), 10.238(\mathrm{~s}, 1 \mathrm{H}, \text { azomethine })\end{array}$ \\
\hline VIII & $\begin{array}{l}2.538(\mathrm{~s}, 2 \mathrm{H}, \text { methylene }), 2.529\left(\mathrm{~s}, 6 \mathrm{H},-\mathrm{CH}_{3}\right), 3.555-3.750\left(\mathrm{~s}, 9 \mathrm{H},-\mathrm{OCH}_{3}\right) \text {, } \\
6.502(\mathrm{~s}, 1 \mathrm{H}, \text { pyrimidine }), 6.926\left(\mathrm{~s}, 2 \mathrm{H},-\mathrm{NH}_{2}\right), 7.126-7.868(\mathrm{~m}, 12 \mathrm{H} \text {, aromatic } \\
\text { proton }), 8.149\left(\mathrm{~s}, 2 \mathrm{H} \text {, phenolic } \mathrm{OH} \text { of } \mathrm{R}_{2} \mathrm{Te}\right), 10.239(\mathrm{~s}, 1 \mathrm{H} \text {, azomethine })\end{array}$ \\
\hline
\end{tabular}
$s=$ singlet,$q=$ quartet,$t=$ triplet, $m=$ multiplet

The characterstic downfield shifting of proton signal in all complexes observed in region $5.67 \delta \mathrm{ppm}$ is due to pyrimidine proton in Schiff base clearly indicate the coordination through pyrimidine nitrogen atom ${ }^{87}$. The signal due to $-\mathrm{NH}$ proton is observed around $6.51 \delta \mathrm{ppm}$ which remain intact with slight variation in complexes is due to the proton bounded to nitrogen experience quadruple effect ${ }^{87}$. Thus salicylidene-trimethoprim act as a tridentate $-\mathrm{N},-\mathrm{N},-\mathrm{O}$ chelating ligand in Sal-TMP.ArTeCl $\mathrm{C}_{2}$ and Sal-TMP. $\mathrm{Ar}_{2} \mathrm{TeCl}$ complexes giving six coordinate tellurium having distorted octahedral geometry in these complexes as predicated from IR studies as well. The proposed structures are as given below (Figure 2). 


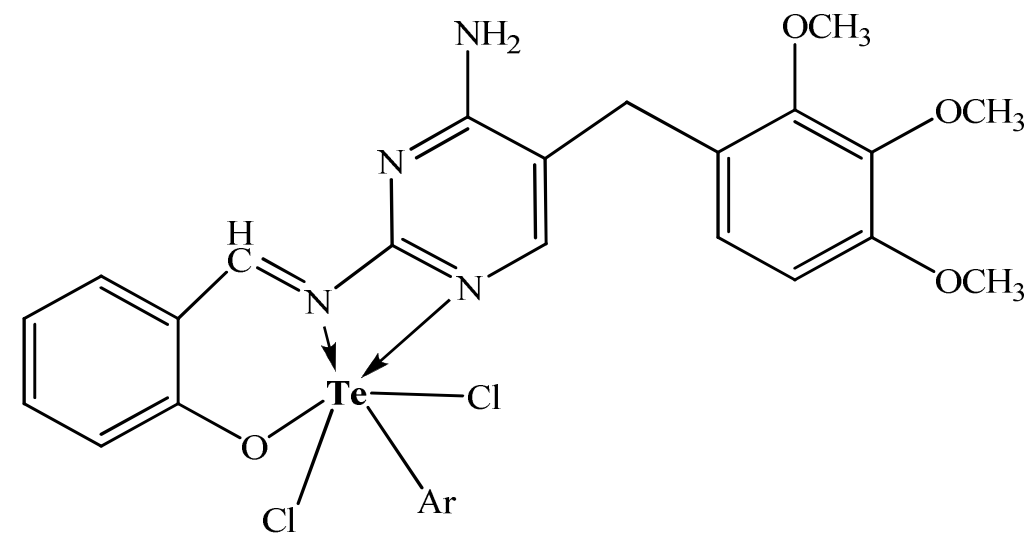<smiles></smiles>

Sal-TMP.Ar ${ }_{2} \mathrm{TeCl}$

$\mathrm{Ar}=p$-methoxyphenyl, $p$-ethoxyphenyl, $p$-hydroxyphenyl and 3-methyl-4-hydroxyphenyl

Figure 2. Proposed structures of complexes

\section{Biological Activity}

The salicylidene-trimethoprim Schiff base (Sal-TMP) and newly synthesized aryltellurium(IV) schiff base complexes were evaluated for their antimicrobial activity in vitro against Gram +ve bacteria (S. aureus ATCC 11632 and B. cereus MTCC 7350), Gram -ve bacteria (E. coli ATCC 35218, P. aeruginosa ATCC 23564, S. typhi ATCC 15499 and $P$. rettgeri DRDE) and fungal strains (A. niger, A. fumigates and A. flavus) by tube dilution method $^{88}$. Dilution of test and standard compounds were prepared double strength nutrient broth- I.P (Antibacterial) and Sabouraud Dextrose Broth -I.P (Antifungal) ${ }^{89}$. The samples were incubated at $37 \pm 1^{\circ} \mathrm{C}$ for $24 \mathrm{~h}$ (bacteria), $25 \pm 1^{\circ} \mathrm{C}$ for 7 days (A. niger), $30 \pm 1^{\circ} \mathrm{C}$ for 15 days (A. flavus), $35 \pm 1{ }^{\circ} \mathrm{C}$ for $72 \mathrm{~h}$ (A. fumigates) respectively and results were recorded in terms of MIC (The lowest concentration of test substances which inhibited values are presented in the Table 4.

The data show that the Sal-TMP complexes of aryltellurium(IV) exhibit more antimicrobial activity towards bacteria as compared to fungii. The antibacterial activity shows following trend. 
Sal-TMP.Ar $\mathrm{ArCl}_{2} \mathrm{Te}$ Sal-TMP.ArTeCl $\mathrm{Cl}_{2} \approx$ Sal-TMP Schiff base

Sal-TMP. $\mathrm{Ar}_{2} \mathrm{TeCl}$ and Sal-TMP.ArTeCl $\mathrm{Cl}_{2}$ complexes have activity towards $S$. typhi and more effectively against $B$. cereus but Schiff base does not show activity against these bacterial strains. Schiff base and its complexes show almost similar activity against fungal strains.

Table 4. Minimum Inhibitory Concentration, MIC, $\mu \mathrm{g} / \mathrm{mL}$; (-) Resistant

\begin{tabular}{|c|c|c|c|c|c|c|c|c|c|}
\hline \multirow[b]{2}{*}{ 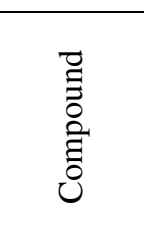 } & \multicolumn{6}{|c|}{ Bacteria strains } & \multicolumn{3}{|c|}{ Fungal strains } \\
\hline & $\begin{array}{c}S . \\
\text { aureus } \\
\text { (ATCC } \\
11632 \text { ) }\end{array}$ & $\begin{array}{l}\text { S. typhi } \\
\text { (ATCC } \\
15499 \text { ) }\end{array}$ & $\begin{array}{c}P . \\
\text { aeruginosa } \\
\text { (ATCC } \\
\text { 23564) }\end{array}$ & $\begin{array}{c}E . \\
a \quad c o l i \\
(\mathrm{ATCC} \\
35218)\end{array}$ & $\begin{array}{c}\text { B. cereus } \\
\text { (MTCC } \\
7350)\end{array}$ & $\begin{array}{c}P . \\
\text { rettgeri } \\
\text { (DRDE } \\
\text { strain) }\end{array}$ & A. niger & $\begin{array}{c}A . \\
\text { fumigates }\end{array}$ & $\begin{array}{l}\text { A. } \\
\text { flavus }\end{array}$ \\
\hline Sal-TMP & 2.5 & - & 1.25 & 5.0 & - & - & 20 & 5.0 & 1.25 \\
\hline I & 2.5 & - & 1.25 & - & 0.625 & 5.0 & - & - & - \\
\hline II & 5.0 & - & 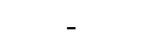 & - & 1.25 & 2.5 & 20 & 5.0 & 1.25 \\
\hline III & - & 20 & 10 & 5.0 & 1.25 & - & 5.0 & - & - \\
\hline IV & 1.25 & 2.5 & 1.25 & 5.0 & - & - & 5.0 & 10 & 5.0 \\
\hline V & - & 20 & 10 & 5.0 & 1.25 & - & 20 & - & - \\
\hline VI & 1.25 & 2.5 & 1.25 & 5.0 & - & - & - & 5.0 & - \\
\hline VII & 1.25 & - & 5.0 & 1.25 & 0.625 & 5.0 & - & 10 & - \\
\hline VIII & 2.5 & - & 1.25 & 5.0 & - & - & 20 & 5.0 & 1.25 \\
\hline
\end{tabular}

\section{Conclusion}

Aryltellurium(IV) and diaryltellurium(IV) dichlorides upon reaction with Schiff base(Sal-TMP) derived from salicylaldehyde and trimethoprim yield new complexes of tellurium(IV). The synthesized complexes were characterized by elemental analyses, conductance measurement, IR and ${ }^{1} \mathrm{H}$ NMR spectral studies. The analytical data suggest that the Sal-TMP Schiff base complexes have 1:1 stoichiometry. The Schiff bases (Sal-TMP) in these complexes functions as a uninegative tridentate ligand through azomethine nitrogen, phenolic oxygen after deprotonation and pyrimidine ring nitrogen atoms. Based on these studies, distorted octahedral geometry with two chelating rings has been assigned to these complexes. The complexes have been observed to possess substantial antimicrobial activity especially against bacteria.

\section{Acknowledgement}

The authors are grateful to M. D. University, Rohtak for providing the necessary facilities. One of the authors (Deepak) is also thankful to UGC New Delhi for providing fellowship. We also thank SAIF, Panjab University Chandigarh for providing the CHN analyses and spectral data.

\section{References}

1. Burchall J J, Corcoran J W and Hahn F E, Eds., Antibiotics, Springer, New York, NY, USA, $1975,3$.

2. Iqbal M S, Khan A H, Loothar B A and Bukhari I H, Med Chem Rev., 2009, 18, 31-42.

3. Blower P J, Аnnu Rep Prog Chem Sect A, 1999, 95, 631-655; DOI:10.1039/A808038K

4. Dhahir S A, Al-Sahib S A, Al Razaq W A and Kadhim N J, Baghdad Sci J., 2010, 7(1), 621.

5. Dr. Karl Thomae Gmb H, (Biberach an der Riss. DE), United States Patent. 4829058.

6. EL-Mossalamy E H, AL-Thabati S A, ALNomaiser F M, Commun Fac Sci Univ Ank Series B, 2005, 51(2), 21-30 
7. Sinha D, Tiwari A K, Singh S, Shukla G, Mishra P, Chandra H and Mishra A K, Eur J Med Chem., 2008, 43(1), 160-165; DOI:10.1016/j.ejmech.2007.03.022

8. Crowe A J, Smith P J and Atassi G, Chem Biol Interact., 1980, 32(1-2), 171-178; DOI:10.1016/0009-2797(80)90075-7

9. Wang M, Wang L F, Li Y Z, Li Q X, Xu Z D and Qu D M, Trans Met Chem., 2001, 26(3), 307-310; DOI:10.1023/A:1007159301849

10. Zishen Wu, Ziqi Gui and Zhenhuan Yen, Synth React Inorg Met Org Chem., 1990, 20(3), 335-344.

11. Desai S B, Desai P B and Desai K R, Hetrocycl Commun., 2001, 7(1), 83-90; DOI:10.1515/HC.2001.7.1.83

12. Dhumwad S D, Gudasi K B and Goudar T R, Indian J Chem., 1994, 33A, 320-324.

13. Przybylski P, Huczynski A, Pyta K, Brzezinski B and Bartl F, Curr Org Chem., 2009, 13(2), 124-148; DOI:10.2174/138527209787193774

14. Pandeya S N, Sriram D, Nath G and De Clercq E, Pharm Acta Hely., 1999, 74(1), 7-11.

15. Karthikeyan M S, Parsad D J, Poojary B, Bhat K S, Holla B S and Kumari N S, Bioorg. Med. Chem., 2006, 14(22), 7482-7489; DOI:10.1016/j.bmc.2006.07.015

16. Holla B S, Akberali P M and Shivananda M K, II Farmaco, 2001, 56(12), 919-927; DOI:10.1016/S0014-827X(01)01124-7

17. Jarrahpour A, Khalili D, De Clercq E, Salmi C and Brunel J M, Molecules, 2007, 12(8), 1720-1730; DOI:10.3390/12081720

18. Da Silva C M, da Silva D L, Modolo L V, Alves R B, de Resende, M A, Martins C V B and de Fatima A J, Adv Res., 2011, 2, 1-8; DOI:10.1016/j.jare.2010.05.004

19. Singh H, Yadav L D S and Mishra S B S, J Inorg Nucl Chem., 1981, 43(7), 17011704; DOI:10.1016/0022-1902(81)80367-3

20. Saravanan G, Pannerselvam P and Prakash C R, J Adv Pharm Techn Res., 2010, 1(3), 320-325; DOI:10.4103/0110-5558.72426

21. Panneerselvam P, Nair R R, Vijayalakshmi G, Subramanian E H and Sridhar S K, Eur. J. Med. Chem. 2005, 40, 225-229; DOI:10.1016/j.ejmech.2004.09.003

22. Singh N K and Singh S B, Indian J Chem., 2001, 40A, 1070-1075.

23. Walsh O M, Meegan M J, Prendergast R M, Nakib T A, Eur J Med Chem., 1996, 31, 989-1000; DOI:10.1016/S0223-5234(97)86178-8

24. Odabasoglu M, Arslan F, Ölmez H and Büyükgüngör O, Dyes and Pigments, 2007, 75(3), 507-515; DOI:10.1016/j.dyepig.2006.06.033

25. Vicini P, Geronikaki A, Incerti M, Busonera B, Poni G, Cabras C A and Colla P L, Bioorg Med Chem., 2003, 11(23), 4785-4789; DOI:10.1016/S09680896(03)00493-0

26. Pandeya S N, Sriram D, Nath G and DeClercq E, Eur J Pharm Sci., 1999, 9, 25-31.

27. Samadhiya S and Halve A, Orient J Chem., 2001, 17, 119-122.

28. Celik Ö, Ulusoy M, Tas E and Ide S, Anal Sciences, 2007, 23, x185.

29. Mahmoud M R, El-Gyar S A, Mousrafa A A and Shaker A, Polyhedron, 1987, 6(5), 1017-1020; DOI:10.1016/S0277-5387(00)80947-X

30. Qing-Yu H, Zheng-Hua M and Ya-Me Z, J Coord Chem., 1990, 21(3), 199-207; DOI:10.1080/00958979009409716

31. Cozzi P G, Chem Soc Rev., 2004, 33, 410-421; DOI:10.1039/B307853C

32. Tarafder M T H, Jin KT, Crouse K A, Ali A M. Yamin, B $M$ and Fun H K, Polyhedron, 2002, 21(25-26), 2547-2554; DOI:10.1016/S0277-5387(02)01188-9

33. Pandeya S N, Sriram D, Clercq E DE., Pannecouque C and Witvrouw M, Indian J Pharm Sci., 1998, 60(4), 207-212. 
34. Fleischman S H and Brooks C L, Proteins: Structure, Function and Genetics, 1990, 7, 52-61.

35. Suling W J, Reynolds R C, Barrow E W, Wilson L N, Piper J R and Barrow W W, J Antimicrobial Chemother., 1998, 42(6), 811-815; DOI:10.1093/jac/42.6.811

36. Gulcan M, Sonmez M and Berber I, Turkey J Chem., 2012, 36(1), 189-200.

37. Raman N, Kulandaisamy A and Jeyasubramanian K, Synth React Inorg Met Org Chem., 2001, 31(7), 1249-1270.

38. Wynne K J and Pearson P S, Inorg Chem., 1971, 10(12), 2735-2739; DOI:10.1021/ic50106a022

39. Wynne K J and Pearson P S, J Chem Soc Commun., 1970, 556-557; DOI:10.1039/C2970000556B

40. Wynne K J, Clark A J and Berg M, J Chem Soc Dalton Trans., 1972, 2370-2374; DOI:10.1039/DT9720002370

41. Clark E R, Collet A J and Naik D G, J Chem Soc Dalton Trans., 1973, 1961-1962; DOI:10.1039/DT9730001961

42. Berg M C, Diss Abstr Int., 1972, 33, 2982.

43. Srivastava T N, Singh M and Singh H B, Indian J Chem., 1982, 21A, 307-309.

44. Srivastava T N, Srivastava R C and Srivastava M, Indian J Chem., 1982, 21A, 539.

45. Srivastava T N, Srivastava R C and Srivastava V K, J Indian Chem Soc., 1983, 60, 891-892.

46. Garad M V, Polyhedron, 1985, 4(8), 1353-1355; DOI:10.1016/S0277-5387(00)86963-6

47. Verma K K and Reena, Synth React Inorg Met-Org Chem., 1999, 29(3), 499-512; DOI:10.1080/00945719909349465

48. Verma K K, Dahiya R and Soni D, Synth React Inorg Met Org Chem., 1999, 29(6), 1033-1052; DOI:10.1080/00945719909349509

49. Verma K K and Dahiya R, Synth React Inorg Met Org Chem., 1999, 29(7), 12991314; DOI:10.1080/00945719909349529

50. Verma K K and Reena, Phosphorus, Sulfur Silicon Related Elements, 1999, 148(1), 227-234; DOI:10.1080/10426509908037013

51. Verma K K and Seema, Int J Chem Sci., 2008, 6, 371-380.

52. Srivastava S, Soni D K and Gupta H S, J Indian Chem Soc., 1996, 73, 255.

53. Narwal J K, Chhabra S, Malik R K, Garg S and Verma K K, Oriental J Chem., 2013, 29, 1339-1349.

54. Chhabra S and Verma K K, J Chem Pharm Res., 2010, 2(4), 569-575.

55. Vogel A I, A Test Book of Organic Chemistry, $3^{\text {rd }}$ Edn., Longman, London, 1975.

56. Weissberger A, Ed., Technique of Organic Chemistry, Vol. 7, $2^{\text {nd }}$ Edn., Interscience Publishers, Inc. N. Y., 1967.

57. Morgan G T and Kellet R E, J Chem Soc., 1926, 1080-1088; DOI:10.1039/JR9262901080

58. Petragnani $\mathrm{N}$ and Stefani $\mathrm{H}$ A, Tellurium in Organic Chemistry, $2^{\text {nd }}$ Edn., Academic Press, London, 2007, 67, 76.

59. Bergman J, Tetrahedron, 1972, 28(12), 3323-3331; DOI:10.1016/S0040-4020(01)93674-9

60. Khandelwal B L, Kumar K and Berry F J, Inorg Chim Acta, 1981, 99(2), 135-137; DOI:10.1016/S0020-1693(00)87958-X

61. Berry F J, Kustan E H, Roshani M and Smith B C, J Organometal Chem., 1975, 99(1), 115-117; DOI:10.1016/S0022-328X(00)86367-6

62. Khandelwal B L, Kumar K and Raina K, Synth React Inorg Met Org Chem., 1981, 11, 65-78.

63. Geary W J, Coord Chem Rev., 1971, 7(1), 81-122; DOI:10.1016/S0010-8545(00)80009-0 
64. Greenwood N N, Straughan B P and Wilson A E, J Chem Soc A, 1968, 2209-2212; DOI: $10.1039 / \mathrm{J} 19680002209$

65. Srivastava K P, Singh A and Singh S K, IOSR J Appl Chem., 2014, 7(4), 16-23; DOI:10.9790/5736-07411623

66. Baker A W and Shulgin A T, J Am Chem Soc., 1959, 81(7), 1523-1529; DOI:10.1021/ja01516a001

67. Freedman H H, J Am Chem Soc., 1961, 83(13), 2900-2905; DOI:10.1021/ja01474a026

68. Flett M St C, Spectrochim Acta, 1957, 10(1), 21-37; DOI:10.1016/0371-1951(57)80160-X

69. Bellamy I J, Infrared Spectra of Complex Molecules, Chapman and Hall Ltd. London, 1975.

70. Matijević-Sosa J, Vinković M and Vikić-Topić D, Croatica Chemica Acta., 2006, 79(3), 489-495.

71. Mishra A P and Soni M, Metal Baesd Drugs, 2008, 71, 243; DOI:10.1155/2008/875410

72. Aminabhavi T M, Biradar N S and Patil C S, Inorganica Chimica Acta., 1983, 78, 107-111; DOI:10.1016/S0020-1693(00)86498-1

73. Biradar N S, Aminabhavi T M and Patil C S, Inorganica Chimica Acta., 1983, 78, 47-50; DOI:10.1016/S0020-1693(00)86487-7

74. Tumer M, Celik C, Koksal H and Serin S, Transition Metal Chem., 1999, 24(5), 525532; DOI:10.1023/A:1006982622965

75. Gwaram N S, Mohd Ali H, Khaledi H, Abdulla M A, Hamid A, Hadi A, Kawi Lin T, Ching C L and Ooi C L, Molecules, 2012, 17(5), 5952;

DOI:10.3390/molecules17055952

76. Rudzinski W E and Aminabhavi T M, Inorganica Chimica Acta., 1982, 67, 177-182; DOI:10.1016/S0020-1693(00)85061-6

77. Osowole A A, Wakil S M and Alao O K, World Appl Sci J., 2015, 33(2), 336-342; DOI:10.5829/idosi.wasj.2015.33.02.22206

78. UR-Rehman S, Faiz A and Nawaz R, J Chil Chem Soc., 2013, 58(1), 1624-1627; DOI:10.4067/S0717-97072013000100026.

79. Mohamed G G and Abd El-Wahab Z H, J Thermal Anal Calorimetry, 2003, 73(1), 347-359; DOI:10.1023/A:1025126801265

80. Verma K K, Soni D and verma, Phosphorus, Sulfur Silicon, 2000, 166(1), 231-241; DOI:10.1080/10426500008076544

81. Pant B C, McWhinnie W R and Dance N S, J Organmetal Chem., 1973, 63, 305-310; DOI:10.1016/S0022-328X(73)80043-9

82. Srivastava T N, Singh J D, Indian J. Chem., 1987, 26A, 260.

83. Chauhan S, Garg S and Verma K K, Chem Sci Trans., 2016, 5(2), 431-441; DOI:10.7598/cst2016.1193

84. Kulkarani Y D, Srivastava S, Abdi S H R and Athar M, Synth React Inorg Met Org Chem., 1985, 15(8), 1043-1059; DOI:10.1080/00945718508060634

85. Chauhan S, Garg S and Verma K K, Res J Pharm Biol Chem Sci., 2016, 7(2), 265-274.

86. Aminabhavi T M and Rudzinski W E, Inorganica Chimica Acta., 1983, 76, L131L134; DOI:10.1016/S0020-1693(00)81478-4

87. Aminabhavi T M and Rudzinski W E, Inorganica Chimica Acta., 1983, 78, 51-55; DOI:10.1016/S0020-1693(00)86488-9

88. Cappuccino J C, Sherman N, Microbiology- A Laboratory Manual, Addison Wesley, California, 1999, 263.

89. Pharmacopoeia of India, Volume 1, Controller of Publications, Ministry of Health Department, Government of India, New Delhi, 2007, 37. 


\title{
Synthesis, Spectral and Biological Studies of Some Salicylidene-Trimethoprim Schiff Base Complexes of Aryltellurium(IV)
}

\author{
DEEPAK, K. K. VERMA and SAPANA GARG* \\ Department of Chemistry, Maharshi Dayanand University, Rohtak-124001, India \\ sapanagarg1511@gmail.com
}

Received 14 February 2017 / Accepted 2 March 2017

\begin{abstract}
A novel monobasic tridentate Schiff base salicylidene-trimethoprim, Sal-TMP, synthesized from trimethoprim and salicylaldehyde, form stable complexes with aryltellurium(IV) trichlorides and diaryltellurium(IV) dichlorides of the type Sal-TMP.ArTeCl ${ }_{2}$ and $\mathrm{Sal}_{-} \mathrm{TMP}_{\mathrm{Ar}} \mathrm{AeCl}$ (where $\mathrm{Ar}=p$-methoxyphenyl, $p$-ethoxyphenyl, $p$-hydroxyphenyl and 3-methyl-4-hydroxyphenyl). These have been characterized by elemental analyses, molar conductance, IR and ${ }^{1} \mathrm{H}$ NMR spectroscopy. The spectral studies predict the bonding of ligand through phenolic oxygen of Schiff base after deprotonation, nitrogen of the azomethine group and pyrimidine nitrogen to give hexacoordinated tellurium(IV) complexes. The complexes have also been screened for their antimicrobial activities against various bacteria and fungi organisms.
\end{abstract}

Keywords: Salicylidene-trimethoprim Schiff base, Aryltellurium(IV), Diaryltellurium(IV), Antibacterial, Antifungal activities

\section{Introduction}

Trimethoprim and its derivatives are broad spectrum antimicrobial agents with anti-parasitic activity $^{1-6}$ and were first described by Roth and coworker ${ }^{5}$. Schiff bases, also known as azomethine due to presence of $-\mathrm{C}=\mathrm{N}$ - group, play important role in biological system, such as anticancer $^{7-12}$, antibacterial ${ }^{13-15}$, antiviral ${ }^{16-18}$, antifungal ${ }^{19-21}$ and other biological properties $^{22-27}$ and also have been extensively used as ligands in coordination chemistry because of their excellent donor abilities as chelating agent ${ }^{28-32}$. Medicinal chemists have reported new derivatives of trimethoprim(TMZ) ${ }^{33-35}$ including the Schiff base derived from salicylaldehyde $2-4,36,37$.

Also, aryltellurium(IV) chlorides are known ${ }^{38-54}$ to act as Lewis acids and form complexes with several N-, O- and S- donor bases. In view of this, we herein report some new complexes derived from aryltellurium(IV) trichlorides, $\mathrm{RTeCl}_{3}$ and diaryltellurium(IV) dichlorides, $\mathrm{R}_{2} \mathrm{TeCl}_{2}$ with salicylidene-trimethoprim Schiff base(Sal-TMP). 


\section{Experimental}

All preparations were carried out under dry $\mathrm{N}_{2}$ atmosphere and the solvents used were purified by standard method ${ }^{55,56}$ before use. The purity of compounds was checked by thin layer chromatography using silica gel-G (Merck). Melting points were determined in open capillary tube and are uncorrected.

Carbon, hydrogen and nitrogen analyses were obtained microanalytically from SAIF, Panjab University Chandigarh on a ThermoFinnigan CHNS analyser. Conductivity was measured in DMSO at $25 \pm 2{ }^{\circ} \mathrm{C}$ with dip type conductivity cell on a microprocessor based conductivity bridge type MICROSIL.

IR $\left(4000-400 \mathrm{~cm}^{-1}\right)$ and far IR $\left(400-50 \mathrm{~cm}^{-1}\right)$ spectra were recorded in $\mathrm{KBr} /$ polyethylene pellets on a FT-Infrared spectrophotometer model RZX (Perkin Elmer) at SAIF, Panjab University Chandigarh. Proton magnetic resonance spectra were recorded in DMSO- $\mathrm{d}_{6}$ using TMS as an internal reference on BRUKER AVANCE II 400 NMR spectrometer. The antimicrobial screening was carried out by tube dilution method at Department of Pharmaceutical Sciences, M. D. University, Rohtak, India.

\section{Preparation of aryltellurium(IV) trichlorides and diaryltellurium(IV) dichlorides}

p-Methoxyphenyltellurium(IV) $\quad$ trichloride $^{57,58}, \quad$ bis(p-methoxyphenyl)tellurium(IV) dichloride ${ }^{58,59}, \quad p$-ethoxyphenyltellurium (IV) trichloride ${ }^{60}$, bis( $p$-ethoxyphenyl)tellurium dichloride $^{60} p$-hydroxyphenyltellurium(IV) trichloride ${ }^{61}$, bis( $p$-hydroxyphenyl) tellurium(IV) dichloride $^{61}$, 3-methyl-4-hydroxyphenyltellurium(IV) trichloride $^{62}$ and bis(3-methyl-4hydroxyphenyl)tellurium(IV) dichloride ${ }^{62}$ were prepared by the reactions of $\mathrm{TeCl}_{4}$ with anisole, phenetole, phenol, $o$-cresol respectively, by the methods reported in the literature ${ }^{57-62}$.

\section{Preparation of salicylidene-trimethoprim Schiff base (Sal-TMP)}

Equimolar quantity of saturated methanolic solution of drug and salicylaldehyde were mixed thoroughly. To this mixture $0.1 \%$ methanolic $\mathrm{KOH}$ was added to adjust the $\mathrm{pH}$ of the solution between 7-8 and was refluxed for 2 hours. A clear yellowish-green coloured solution was obtained. After completion of the reaction, the Schiff base ligand was isolated by crystallization after volume reduction by evaporation. The crystalline product was filtered and dried under vacuum and kept in desiccator over $\mathrm{P}_{4} \mathrm{O}_{10}$ until further use. Yield $=$ $80 \%$, M.pt.(decomp.) $=188-190{ }^{\circ} \mathrm{C}$. Analysis (Calculated) $\mathrm{C}_{21} \mathrm{H}_{22} \mathrm{~N}_{4} \mathrm{O}_{4}: \mathrm{C}(63.95), \mathrm{H}(5.62)$ and $\mathrm{N}(14.20)$; Found: $\mathrm{C}(63.50), \mathrm{H}(5.27)$ and $\mathrm{N}(13.99)$.

\section{Preparation of salicylidene-trimethoprim complexes of aryltellurium(IV) trichlorides} and diaryltellurium(IV) dichlorides

Aryltellurium(IV) trichlorides, $\mathrm{ArTeCl}_{3}$ and diaryltellurium(IV) dichlorides $\mathrm{Ar}_{2} \mathrm{TeCl}_{2}$ (Ar= $p$-methoxyphenyl, $p$-ethoxyphenyl, $p$-hydroxyphenyl and 3-methyl-4-hydroxyphenyl), when reacted with sodium salt of salicylidene-trimethoprim in equimolar ratio, yield SalTMP.ArTeCl $\mathrm{Cl}_{2}$ and Sal-TMP.Ar $\mathrm{TeCl}_{2} \mathrm{Type}$ complexes.

Sodium salt of the ligand was prepared by reacting equimolar (1:1) quantity of sodium metal and Schiff base in methanol. The solvent was distilled off to obtain sodium salt of Schiff base. Then a methanolic saturated solution of $2 \mathrm{mmol}$ of aryltellurium(IV) trichloride or diaryltellurium(IV) dichloride was added dropwise to suspension of $2 \mathrm{mmol}$ of sodium salt of Schiff base in about $50 \mathrm{~mL}$ benzene under reflux. The reaction mixture was further refluxed for 3-4 hours, cooled and precipitated sodium chloride was filtered off. The filtrate was then concentrated to about one third of original volume under reduced pressure and cooled in an ice bath to obtain coloured product. This was filtered, washed with benzene + methanol (1:1) and dried in vacuum desiccator over $\mathrm{P}_{4} \mathrm{O}_{10}$. 


\section{Results and Discussion}

$\mathrm{TeCl}_{4}$ when heated with anisole ${ }^{57-59}$, phenetole ${ }^{60}$, phenol ${ }^{61}, o$-cresol ${ }^{62}$ (Ar-H) appears to undergo Friedel-Crafts type condensation reaction whereby $\mathrm{TeCl}_{3}{ }^{+}$unit attacks a position para to the methoxy/ethoxy/hydroxy groups in the aromatic rings, thus resulting in the formation of aryltellurium(IV) trichlorides and diaryltellurium(IV) dichlorides.

$$
\begin{gathered}
\mathrm{Ar}-\mathrm{H}+\mathrm{TeCl}_{4} \longrightarrow \mathrm{ArTeCl}_{3}+\mathrm{HCl} \\
2 \mathrm{Ar}-\mathrm{H}+\mathrm{TeCl}_{4} \longrightarrow \mathrm{Ar}_{2} \mathrm{TeCl}_{2}+2 \mathrm{HCl}
\end{gathered}
$$

Preparation of salicylidene-trimethoprim Schiff base (Sal-TMP) by the reaction of trimethoprim drug and salicylaldehyde can be represented by following equations.

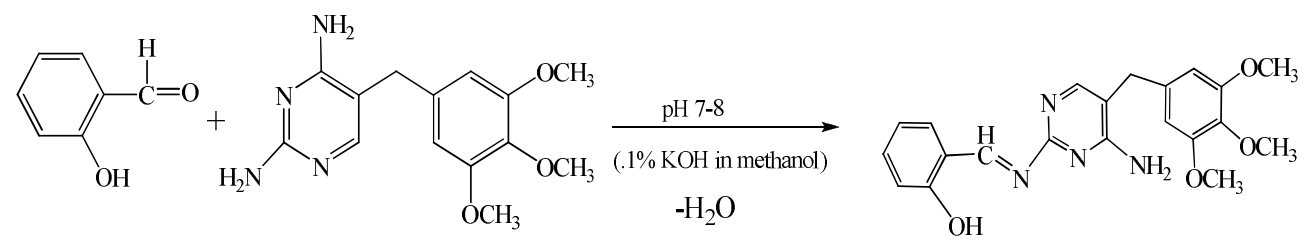

Sodium salt of salicylidene-trimethoprim Schiff base (Sal-TMP) reacts with aryltellurium(IV) trichlorides and diaryltellurium(IV) dichlorides in 1:1 molar ratio to yield the corresponding aryltellurium(IV) complexes.

$$
\begin{aligned}
& \mathrm{Sal}-\mathrm{TMP}+\mathrm{ArTeCl}_{3} \underset{\mathrm{NaCl}}{\stackrel{\mathrm{Na} / \mathrm{CH}_{3} \mathrm{OH}}{\longrightarrow}} \text { (Sal-TMP).ArTeCl } 2 \\
& \mathrm{Sal}-\mathrm{TMP}+\mathrm{Ar}_{2} \mathrm{TeCl}_{2} \underset{-\mathrm{NaCl}}{\stackrel{\mathrm{Na} / \mathrm{CH}_{3} \mathrm{OH}}{\longrightarrow}} \text { (Sal-TMP). } \mathrm{Ar}_{2} \mathrm{TeCl}_{2}
\end{aligned}
$$

All the tellurium(IV) complexes are coloured, crystalline solids, stable at room temperature and non-hygroscopic in nature. The complexes have been analysed for their tellurium, chlorine, carbon, hydrogen and nitrogen contents and the data along with their physical properties and yields are presented in Table 1.

\section{Conductance studies}

Molar conductance $\left(\Lambda_{M}\right)$ data for the complexes in DMSO are complied in Table 1. The $\Lambda_{M}$ value at $c a .10^{-3} \mathrm{M}$ for aryltellurium(IV) complexes in DMSO lie in the range 27.36-91.83 S $\mathrm{cm}^{2} \mathrm{~mol}^{-1}$ which predict the non electrolyte to $1: 1$ electrolyte $^{63,64}$ type behaviour of these complexes in DMSO, probably due to ionization into $\mathrm{ArTeCl}$.Sal- $\mathrm{TMP}^{+} / \mathrm{Ar}_{2} \mathrm{Te}$.Sal-TMP ${ }^{+}$ and $\mathrm{Cl}^{-}$in DMSO. This conductance behavoiur of tellurium(IV) salicylidene-trimethoprim Schiff base complexes is different from those of transition metal complexes ${ }^{65}$, which are reported to be non-electrolytes. The higher $\Lambda_{\mathrm{M}}$ values for some complexes may be due to steric factors and donor behaviour of DMSO to result in probable dissociation into solvated cation and $\mathrm{L}^{-}$along with $\mathrm{Cl}^{-}$in DMSO.

\section{Infrared spectra}

The IR data of Schiff base and its tellurium(IV) complexes are listed in Table 2. The spectra of Sal-TMP Schiff base complexes are quite complex and an attempt has therefore been made to identify the donor sites by comparing the spectra of complexes with parent ligand and $\mathrm{ArTeCl}_{3} / \mathrm{Ar}_{2} \mathrm{TeCl}_{2}$. 
Table 1. Analytical data, molar conductance and physical properties of salicylidene-trimethoprim Schiff base (Sal-TMP) complexes of tellurium(IV)

\begin{tabular}{|c|c|c|c|c|c|c|c|c|c|c|}
\hline \multirow[b]{2}{*}{$\begin{array}{l}\text { Compound } \\
\text { No. }\end{array}$} & \multirow[b]{2}{*}{$\begin{array}{l}\text { Complex } \\
\text { (Ar) }\end{array}$} & \multirow{2}{*}{$\begin{array}{c}\text { Empirical } \\
\text { formula } \\
\text { (Formula Wt.) }\end{array}$} & \multirow[b]{2}{*}{$\begin{array}{l}\text { Colour } \\
\text { (Yield, \%) }\end{array}$} & \multirow{2}{*}{$\begin{array}{l}\text { M. Pt. } \\
{ }^{\circ} \mathrm{C} \\
\text { dec. }\end{array}$} & \multicolumn{5}{|c|}{ Analyses \% Found (Calculated) } & \multirow{2}{*}{$\begin{array}{l}\Lambda_{\mathrm{M}} \text { at } c a \\
10^{-3} \mathrm{M} \mathrm{S} \\
\mathrm{cm}^{2} \mathrm{~mol}^{-1} \\
\text { in DMSO }\end{array}$} \\
\hline & & & & & $\mathrm{C}$ & $\mathrm{H}$ & $\mathrm{N}$ & $\mathrm{Te}$ & $\mathrm{Cl}$ & \\
\hline $\begin{array}{l}\text { Schiff } \\
\text { Base }\end{array}$ & Sal-TMP & $\mathrm{C}_{21} \mathrm{H}_{22} \mathrm{~N}_{4} \mathrm{O}_{4}$ & $\begin{array}{l}\text { Yellowish } \\
\text {-green }(80)\end{array}$ & $188-190$ & $\begin{array}{c}63.50 \\
(63.95)\end{array}$ & $\begin{array}{c}5.27 \\
(5.62)\end{array}$ & $\begin{array}{l}13.99 \\
(14.20)\end{array}$ & - & - & - \\
\hline I & $\begin{array}{l}\text { (Sal-TMP).ArTeCl }{ }_{2} \\
\text { (p-methoxyphenyl) }\end{array}$ & $\begin{array}{c}\mathrm{C}_{28} \mathrm{H}_{28} \mathrm{Cl}_{2} \mathrm{~N}_{4} \mathrm{O}_{5} \mathrm{Te} \\
(699.05)\end{array}$ & $\begin{array}{l}\text { Light cream } \\
(92)\end{array}$ & $230-232$ & $\begin{array}{c}47.88 \\
(48.11)\end{array}$ & $\begin{array}{c}3.99 \\
(4.04)\end{array}$ & $\begin{array}{c}7.75 \\
(8.01)\end{array}$ & $\begin{array}{c}18.07 \\
(18.24)\end{array}$ & $\begin{array}{c}9.89 \\
(10.01)\end{array}$ & 53.19 \\
\hline II & $\begin{array}{c}\text { (Sal-TMP).ArTeCl } \\
\text { (p-ethoxyphenyl) }\end{array}$ & $\begin{array}{c}\mathrm{C}_{29} \mathrm{H}_{30} \mathrm{Cl}_{2} \mathrm{~N}_{4} \mathrm{O}_{5} \mathrm{Te} \\
(713.08)\end{array}$ & $\begin{array}{c}\text { Cream } \\
(85)\end{array}$ & $258-260$ & $\begin{array}{c}48.12 \\
(48.85)\end{array}$ & $\begin{array}{c}3.87 \\
(4.24)\end{array}$ & $\begin{array}{l}7.52 \\
(7.86)\end{array}$ & $\begin{array}{c}17.38 \\
(17.89)\end{array}$ & $\begin{array}{c}9.52 \\
(9.81)\end{array}$ & 52.88 \\
\hline III & $\begin{array}{c}(\mathrm{Sal}-\mathrm{TMP}) \cdot \mathrm{ArTeCl}_{2} \\
(p \text {-hydroxyphenyl })\end{array}$ & $\begin{array}{c}\mathrm{C}_{27} \mathrm{H}_{26} \mathrm{Cl}_{2} \mathrm{~N}_{4} \mathrm{O}_{5} \mathrm{Te} \\
(685.03)\end{array}$ & $\begin{array}{l}\text { Dark cream } \\
\quad(94)\end{array}$ & $208-210$ & $\begin{array}{c}46.78 \\
(47.34)\end{array}$ & $\begin{array}{c}3.50 \\
(3.83)\end{array}$ & $\begin{array}{c}7.88 \\
(8.18)\end{array}$ & $\begin{array}{c}18.28 \\
(18.63)\end{array}$ & $\begin{array}{c}9.98 \\
(10.21)\end{array}$ & 55.73 \\
\hline IV & $\begin{array}{c}\text { (Sal-TMP).ArTeCl } \\
\text { (3-methyl-4- } \\
\text { hydroxyphenyl) }\end{array}$ & $\begin{array}{c}\mathrm{C}_{28} \mathrm{H}_{28} \mathrm{Cl}_{2} \mathrm{~N}_{4} \mathrm{O}_{5} \mathrm{Te} \\
(699.05)\end{array}$ & $\begin{array}{l}\text { Light } \\
\text { cream } \\
(80)\end{array}$ & $198-200$ & $\begin{array}{c}47.58 \\
(48.11)\end{array}$ & $\begin{array}{c}3.84 \\
(4.04)\end{array}$ & $\begin{array}{c}7.80 \\
(8.01)\end{array}$ & $\begin{array}{c}18.15 \\
(18.24)\end{array}$ & $\begin{array}{c}9.85 \\
(10.01)\end{array}$ & 58.68 \\
\hline $\mathrm{V}$ & $\begin{array}{c}(\mathrm{Sal}-\mathrm{TMP}) \cdot \mathrm{Ar}_{2} \mathrm{TeCl} \\
(p \text {-methoxyphenyl })\end{array}$ & $\begin{array}{c}\mathrm{C}_{35} \mathrm{H}_{35} \mathrm{ClN}_{4} \mathrm{O}_{6} \mathrm{Te} \\
(770.73)\end{array}$ & $\begin{array}{c}\text { Pale yellow } \\
\text { (84) }\end{array}$ & $130-132$ & $\begin{array}{c}53.84 \\
(54.54)\end{array}$ & $\begin{array}{c}4.31 \\
(4.58)\end{array}$ & $\begin{array}{c}7.11 \\
(7.27)\end{array}$ & $\begin{array}{c}16.43 \\
(16.56)\end{array}$ & $\begin{array}{c}4.42 \\
(4.60)\end{array}$ & 91.83 \\
\hline VI & $\begin{array}{c}\text { (Sal-TMP).Ar }{ }_{2} \mathrm{TeCl} \\
(p \text {-ethoxyphenyl) }\end{array}$ & $\begin{array}{c}\mathrm{C}_{37} \mathrm{H}_{39} \mathrm{ClN}_{4} \mathrm{O}_{6} \mathrm{Te} \\
(798.78)\end{array}$ & $\begin{array}{l}\text { Light yellow } \\
\text { (86) }\end{array}$ & $150-152$ & $\begin{array}{c}54.84 \\
(55.63)\end{array}$ & $\begin{array}{c}4.53 \\
(4.92)\end{array}$ & $\begin{array}{c}6.84 \\
(7.01)\end{array}$ & $\begin{array}{c}15.50 \\
(15.97)\end{array}$ & $\begin{array}{c}4.30 \\
(4.44)\end{array}$ & 35.90 \\
\hline VII & $\begin{array}{l}\text { (Sal-TMP).Ar } \mathrm{Ar}_{2} \mathrm{TeCl} \\
(p \text {-hydroxyphenyl) }\end{array}$ & $\begin{array}{c}\mathrm{C}_{33} \mathrm{H}_{31} \mathrm{ClN}_{4} \mathrm{O}_{6} \mathrm{Te} \\
(742.68)\end{array}$ & $\begin{array}{l}\text { Red } \\
(89)\end{array}$ & $146-148$ & $\begin{array}{c}52.84 \\
(53.37)\end{array}$ & $\begin{array}{c}4.01 \\
(4.21)\end{array}$ & $\begin{array}{c}7.27 \\
(7.54)\end{array}$ & $\begin{array}{c}16.89 \\
(17.18)\end{array}$ & $\begin{array}{c}4.52 \\
(4.77)\end{array}$ & 36.00 \\
\hline VIII & $\begin{array}{c}\text { (Sal-TMP). } \mathrm{Ar}_{2} \mathrm{TeCl} \\
\text { (3-methyl-4- } \\
\text { hydroxyphenyl) }\end{array}$ & $\begin{array}{c}\mathrm{C}_{35} \mathrm{H}_{35} \mathrm{ClN}_{4} \mathrm{O}_{6} \mathrm{Te} \\
(770.73)\end{array}$ & $\begin{array}{l}\text { Brown } \\
(78)\end{array}$ & $140-142$ & $\begin{array}{l}53.80 \\
(54.54)\end{array}$ & $\begin{array}{c}4.34 \\
(4.58)\end{array}$ & $\begin{array}{c}7.09 \\
(7.27)\end{array}$ & $\begin{array}{c}16.45 \\
(16.56)\end{array}$ & $\begin{array}{c}4.32 \\
(4.60)\end{array}$ & 27.36 \\
\hline
\end{tabular}


Table 2. Important infrared absorption bands $\left(\mathrm{cm}^{-1}\right)$ of Schiff base (Sal-TMP) and complexes

\begin{tabular}{|c|c|c|c|c|c|c|c|}
\hline Compound & $v_{(O-}$ & $v_{(1}$ & $v_{(C}$ & $v_{(C}$ & $v_{(\mathrm{C}-}$ & $v_{(\mathrm{Te}-\mathrm{N})}$ & $v_{(\mathrm{Te}}$ \\
\hline Sal-TMP & $2836 \mathrm{w}$ & $3317 \mathrm{~m} \mathrm{3119} \mathrm{m}$ & $1636 \mathrm{sh}$ & $1633 \mathrm{w} 1593 \mathrm{~s}$ & $1263 \mathrm{~s}$ & - & - \\
\hline $\bar{I}$ & - & $3323 \mathrm{~m} 3184 \mathrm{~m}$ & $1674 \mathrm{mb}$ & $1644 \mathrm{mb} 1587 \mathrm{~s}$ & $1341 \mathrm{~s}$ & $415 \mathrm{~m}$ & $288 \mathrm{w}$ \\
\hline II & - & $3323 \mathrm{~m} 3170 \mathrm{~m}$ & $1647 \mathrm{sh}$ & **1586 s & $1304 \mathrm{~s}$ & $420 \mathrm{~m}$ & $295 w$ \\
\hline III & 3405 m* & $3323 \mathrm{~m} 3150 \mathrm{~m}$ & $1674 \mathrm{mb}$ & $1641 \mathrm{mb} 1586 \mathrm{~s}$ & $1341 \mathrm{~s}$ & $419 \mathrm{~s}$ & $270 \mathrm{w}$ \\
\hline IV & 3398 m* & $3319 \mathrm{~m} 3172 \mathrm{~m}$ & $1652 \mathrm{mb}$ & 1649 & $1333 \mathrm{~s}$ & $450 \mathrm{~s}$ & $277 \mathrm{w}$ \\
\hline V & - & $3325 \mathrm{~m}$ & 164 & $* * 1$ & $1333 \mathrm{~s}$ & $416 \mathrm{~s}$ & $290 \mathrm{w}$ \\
\hline VI & - & $3320 \mathrm{~m} 3158 \mathrm{~m}$ & $1640 \mathrm{sh}$ & $* * 1590 \mathrm{~s}$ & $1331 \mathrm{~s}$ & $410 \mathrm{~m}$ & $273 w$ \\
\hline VII & $3401 \mathrm{w}^{*}$ & $3324 \mathrm{~m} 3168 \mathrm{~m}$ & $1674 \mathrm{mb}$ & $1643 \mathrm{mb} 1584 \mathrm{~s}$ & $1340 \mathrm{~s}$ & $418 \mathrm{~m}$ & $285 \mathrm{w}$ \\
\hline VIII & $3463 \mathrm{w}^{*}$ & $3304 \mathrm{~m} 3106 \mathrm{~m}$ & $1634 \mathrm{sh}$ & $1643 \mathrm{mb} 1584 \mathrm{~s}$ & $1340 \mathrm{~s}$ & $422 \mathrm{~m}$ & $287 \mathrm{w}$ \\
\hline
\end{tabular}

$s=$ sharp, m=medium, mb=medium broad, sh=shoulder, $w=w e a k$, "Due to phenolic $\mathrm{OH}$ of Rte and $\mathrm{R}_{2} \mathrm{Te}$ moieties; ${ }^{* *}$ band not resolved due to overlapping of band $\boldsymbol{v}_{(C=N)}$

Examination of the Schiff base (Sal-TMP) spectrum shows the presence of the hydrogen bonded conjugated- chelate ring system ${ }^{66-68}$ with its centre at $\sim 2832 \mathrm{~cm}^{-1}$. Thus intramolecular $\mathrm{H}$ - bonding is occurring by means of the formation of a quasi six- membered ring involving the $\mathrm{OH}----\mathrm{N}=\mathrm{C}$ bond.<smiles>C1=Cc2ccccc2O[NH+]=1</smiles>

Figure 1. Hydrogen bonding

Thus band disappear on chelation with aryltellurium(IV) chlorides ${ }^{69}$. Hydrogen bond contributes to planarity of the molecule which helps in chelation ${ }^{70}$. Also an intense ligand band at $1263 \mathrm{~cm}^{-1}$ (phenolic -C-O) in free ligand has shifted to higher frequency side in complexes. All these indicate that the hydroxyl group of salicylaldehyde of Schiff base is involved in coordination with tellurium ${ }^{71-73}$.

In addition, the spectra of the Schiff base shown shoulder at $1636 \mathrm{~cm}^{-1}$ with slightly resolved weak band at $1633 \mathrm{~cm}^{-1}$ and sharp band at $1593 \mathrm{~cm}^{-1}$ assigned to $v_{(\mathrm{C}=\mathrm{N})}$ mode for vibration of azomethine group ${ }^{2,4,65,74-76}$ and $v_{(\mathrm{C}=\mathrm{N}) \text { pyrim. For pyrimidine ring }}{ }^{4,77}$. These shift in aryltellurium Schiff base complexes towards higher and lower value ${ }^{74-79}$ reflecting that ligand coordinate through nitrogen atom of azomethine and pyrimidine ring.

The medium intensity band at $3317 \mathrm{~cm}^{-1}$ and $3119 \mathrm{~cm}^{-1}$ due to $v_{(\mathrm{N}-\mathrm{H})}$ asymmetric and symmetric vibrations respectively indicate the non involvement of the nitrogen atom of $\mathrm{NH}_{2}$ group attached to pyrimidine in coordination. The appearance of new weak bands around $270-295 \mathrm{~cm}^{-1}$ due to $v_{(\mathrm{Te}-\mathrm{O})}^{80-83}$ mode and medium to strong band in the range of 410-422 $\mathrm{cm}^{-1}$ due to $v_{(\mathrm{Te}-\mathrm{N})}$ mode ${ }^{84}$ further supports the involvement of phenolic oxygen (after deprotonation), azomethine and pyrimidine nitrogen atoms of Schiff base in the coordination.

Thus, IR data predict the tridentate nature of Sal-TMP involving azomethine nitrogen atom, phenolic oxygen after deprotonation and pyrimidine ring nitrogen giving rise to twosix and four membered chelate rings with the tellurium centre. 


\section{${ }^{1}$ H NMR spectra}

In order to identify the solution structure of Schiff base (Sal-TMP) and its complexes, ${ }^{1} \mathrm{H}$ NMR spectra were recorded in DMSO- $\mathrm{d}_{6}$ and given in Table 3. The proton resonance of the $\mathrm{OH}$ group at $10.92 \delta \mathrm{ppm}^{76,85}$ in Schiff base due to presence of intramolecular hydrogen bonding ${ }^{74}$ disappear on complexation indicating the involvement of phenolic oxygen in the coordination via deprotonation ${ }^{76}$. The azomethine protons which resonate as a singlet at $10.02 \delta \mathrm{ppm}$, the coordination of azomethine nitrogen to tellurium in the complexes are clearly demonstrated by a downfield shift of the peak ${ }^{86}$.

Table 3. ${ }^{1} \mathrm{H}$ NMR spectral data of Schiff base (Sal-TMP) and complexes in DMSO- $\mathrm{d}_{6}$

\begin{tabular}{|c|c|}
\hline Compound & Chemical Shift, $\delta$ ppm \\
\hline Sal-TMP & $\begin{array}{l}2.525(\mathrm{~s}, 2 \mathrm{H}, \text { methylene }), \quad 3.551-3.745\left(\mathrm{~s}, \quad 9 \mathrm{H},-\mathrm{OCH}_{3}\right), \quad 5.672(\mathrm{~s}, 1 \mathrm{H}, \\
\text { pyrimidine }), 6.508\left(\mathrm{~s}, 2 \mathrm{H},-\mathrm{NH}_{2}\right), 7.526-8.169(\mathrm{~m}, 6 \mathrm{H}, \text { aromatic proton), } \\
10.021(\mathrm{~s}, 1 \mathrm{H}, \text { azomethine }), 10.921(\mathrm{~s}, 1 \mathrm{H}, \mathrm{Schiff} \text { base } \mathrm{OH})\end{array}$ \\
\hline I & $\begin{array}{l}2.539(\mathrm{~s}, 2 \mathrm{H}, \text { methylene }), 3.598-3.840\left(\mathrm{~s}, 12 \mathrm{H},-\mathrm{OCH}_{3}\right), 6.574(\mathrm{~s}, 1 \mathrm{H}, \\
\text { pyrimidine }), 6.926\left(\mathrm{~s}, 2 \mathrm{H},-\mathrm{NH}_{2}\right), 7.383-7.522(\mathrm{~m}, 10 \mathrm{H}, \text { aromatic proton }), \\
10.209(\mathrm{~s}, 1 \mathrm{H}, \text { azomethine })\end{array}$ \\
\hline II & $\begin{array}{l}1.335\left(\mathrm{t}, 3 \mathrm{H},-\mathrm{OCH}_{2} \mathrm{CH}_{3}\right), 2.546(\mathrm{~s}, 2 \mathrm{H}, \text { methylene }), 3.963\left(\mathrm{q}, 2 \mathrm{H},-\mathrm{OCH}_{2} \mathrm{CH}_{3}\right) \text {, } \\
3.515-3.875\left(\mathrm{~s}, 9 \mathrm{H},-\mathrm{OCH}_{3}\right), 6.540(\mathrm{~s}, 1 \mathrm{H}, \text { pyrimidine }), 6.950\left(\mathrm{~s}, 2 \mathrm{H},-\mathrm{NH}_{2}\right), \\
6.993-7.809(\mathrm{~m}, 10 \mathrm{H} \text {, aromatic proton), } 10.193(\mathrm{~s}, 1 \mathrm{H} \text {, azomethine })\end{array}$ \\
\hline III & $\begin{array}{l}2.548(\mathrm{~s}, 2 \mathrm{H}, \quad \text { methylene }), \quad 3.601-3.783\left(\mathrm{~s}, 9 \mathrm{H},-\mathrm{OCH}_{3}\right), \quad 6.569(\mathrm{~s}, 1 \mathrm{H}, \\
\text { pyrimidine }), 6.914\left(\mathrm{~s}, 2 \mathrm{H},-\mathrm{NH}_{2}\right), 7.370-7.787(\mathrm{~m}, 10 \mathrm{H}, \text { aromatic proton }), \\
8.155(\mathrm{~s}, 1 \mathrm{H}, \text { phenolic } \mathrm{OH} \text { of } \mathrm{RTe}), 10.201(\mathrm{~s}, 1 \mathrm{H}, \text { azomethine })\end{array}$ \\
\hline IV & $\begin{array}{l}2.536(\mathrm{~s}, 2 \mathrm{H}, \text { methylene }), 2.527\left(\mathrm{~s}, 3 \mathrm{H},-\mathrm{CH}_{3}\right), 3.587-3.770\left(\mathrm{~s}, 9 \mathrm{H},-\mathrm{OCH}_{3}\right) \text {, } \\
\text { 6.605(s, } 1 \mathrm{H}, \text { pyrimidine }), 6.918\left(\mathrm{~s}, 2 \mathrm{H},-\mathrm{NH}_{2}\right), 7.051-7.671(\mathrm{~m}, 9 \mathrm{H} \text {, aromatic } \\
\text { proton }), 8.251(\mathrm{~s}, 1 \mathrm{H} \text {, phenolic } \mathrm{OH} \text { of } \mathrm{RTe}), 10.239(\mathrm{~s}, 1 \mathrm{H} \text {, azomethine })\end{array}$ \\
\hline V & $\begin{array}{l}2.528(\mathrm{~s}, 2 \mathrm{H}, \text { methylene }), 3.673-3.877\left(\mathrm{~s}, 15 \mathrm{H},-\mathrm{OCH}_{3}\right), 6.516(\mathrm{~s}, 1 \mathrm{H} \text {, } \\
\text { pyrimidine }), 6.882\left(\mathrm{~s}, 2 \mathrm{H},-\mathrm{NH}_{2}\right), 6.910-7.804(\mathrm{~m}, 14 \mathrm{H}, \text { aromatic proton }) \\
10.186(\mathrm{~s}, 1 \mathrm{H}, \text { azomethine })\end{array}$ \\
\hline VI & $\begin{array}{l}\left.\text { 1.363(t, } 6 \mathrm{H},-\mathrm{OCH}_{2} \mathbf{C H}_{3}\right), 2.531(\mathrm{~s}, 2 \mathrm{H}, \text { methylene }), 4.019\left(\mathrm{q}, 4 \mathrm{H},-\mathrm{OCH}_{2} \mathrm{CH}_{3}\right) \text {, } \\
3.555-3.765\left(\mathrm{~s}, 9 \mathrm{H},-\mathrm{OCH}_{3}\right), 6.510(\mathrm{~s}, 1 \mathrm{H}, \text { pyrimidine }), 6.924\left(\mathrm{~s}, 2 \mathrm{H},-\mathrm{NH}_{2}\right) \text {, } \\
\text { 7.524-7.805(m, } 14 \mathrm{H} \text {, aromatic proton }), 10.216(\mathrm{~s}, 1 \mathrm{H} \text {, azomethine })\end{array}$ \\
\hline VII & $\begin{array}{l}2.545(\mathrm{~s}, 2 \mathrm{H}, \quad \text { methylene }), 3.568-3.926\left(\mathrm{~s}, 9 \mathrm{H},-\mathrm{OCH}_{3}\right), 6.554(\mathrm{~s}, 1 \mathrm{H} \text {, } \\
\text { pyrimidine }), 6.884\left(\mathrm{~s}, 2 \mathrm{H},-\mathrm{NH}_{2}\right), 7.488-7.678(\mathrm{~m}, 14 \mathrm{H}, \text { aromatic proton), } \\
8.249\left(\mathrm{~s}, 2 \mathrm{H}, \text { phenolic } \mathrm{OH} \text { of } \mathrm{R}_{2} \mathrm{Te}\right), 10.238(\mathrm{~s}, 1 \mathrm{H}, \text { azomethine })\end{array}$ \\
\hline VIII & $\begin{array}{l}2.538(\mathrm{~s}, 2 \mathrm{H}, \text { methylene }), 2.529\left(\mathrm{~s}, 6 \mathrm{H},-\mathrm{CH}_{3}\right), 3.555-3.750\left(\mathrm{~s}, 9 \mathrm{H},-\mathrm{OCH}_{3}\right) \text {, } \\
6.502(\mathrm{~s}, 1 \mathrm{H}, \text { pyrimidine }), 6.926\left(\mathrm{~s}, 2 \mathrm{H},-\mathrm{NH}_{2}\right), 7.126-7.868(\mathrm{~m}, 12 \mathrm{H} \text {, aromatic } \\
\text { proton }), 8.149\left(\mathrm{~s}, 2 \mathrm{H} \text {, phenolic } \mathrm{OH} \text { of } \mathrm{R}_{2} \mathrm{Te}\right), 10.239(\mathrm{~s}, 1 \mathrm{H} \text {, azomethine })\end{array}$ \\
\hline
\end{tabular}
$s=$ singlet,$q=$ quartet,$t=$ triplet, $m=$ multiplet

The characterstic downfield shifting of proton signal in all complexes observed in region $5.67 \delta \mathrm{ppm}$ is due to pyrimidine proton in Schiff base clearly indicate the coordination through pyrimidine nitrogen atom ${ }^{87}$. The signal due to $-\mathrm{NH}$ proton is observed around $6.51 \delta \mathrm{ppm}$ which remain intact with slight variation in complexes is due to the proton bounded to nitrogen experience quadruple effect ${ }^{87}$. Thus salicylidene-trimethoprim act as a tridentate $-\mathrm{N},-\mathrm{N},-\mathrm{O}$ chelating ligand in Sal-TMP.ArTeCl $\mathrm{C}_{2}$ and Sal-TMP. $\mathrm{Ar}_{2} \mathrm{TeCl}$ complexes giving six coordinate tellurium having distorted octahedral geometry in these complexes as predicated from IR studies as well. The proposed structures are as given below (Figure 2). 


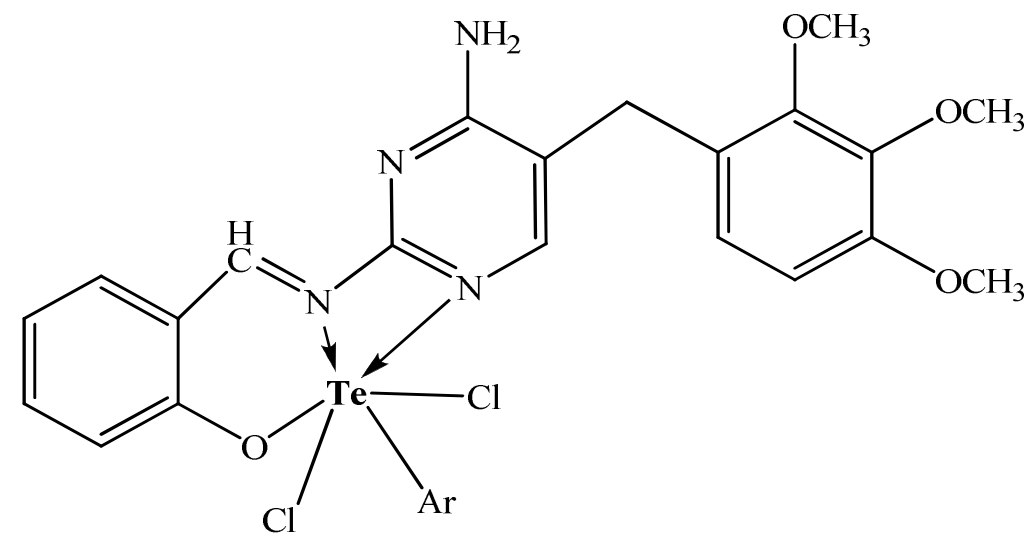<smiles></smiles>

Sal-TMP.Ar ${ }_{2} \mathrm{TeCl}$

$\mathrm{Ar}=p$-methoxyphenyl, $p$-ethoxyphenyl, $p$-hydroxyphenyl and 3-methyl-4-hydroxyphenyl

Figure 2. Proposed structures of complexes

\section{Biological Activity}

The salicylidene-trimethoprim Schiff base (Sal-TMP) and newly synthesized aryltellurium(IV) schiff base complexes were evaluated for their antimicrobial activity in vitro against Gram +ve bacteria (S. aureus ATCC 11632 and B. cereus MTCC 7350), Gram -ve bacteria (E. coli ATCC 35218, P. aeruginosa ATCC 23564, S. typhi ATCC 15499 and $P$. rettgeri DRDE) and fungal strains (A. niger, A. fumigates and A. flavus) by tube dilution method $^{88}$. Dilution of test and standard compounds were prepared double strength nutrient broth- I.P (Antibacterial) and Sabouraud Dextrose Broth -I.P (Antifungal) ${ }^{89}$. The samples were incubated at $37 \pm 1^{\circ} \mathrm{C}$ for $24 \mathrm{~h}$ (bacteria), $25 \pm 1^{\circ} \mathrm{C}$ for 7 days (A. niger), $30 \pm 1^{\circ} \mathrm{C}$ for 15 days (A. flavus), $35 \pm 1{ }^{\circ} \mathrm{C}$ for $72 \mathrm{~h}$ (A. fumigates) respectively and results were recorded in terms of MIC (The lowest concentration of test substances which inhibited values are presented in the Table 4.

The data show that the Sal-TMP complexes of aryltellurium(IV) exhibit more antimicrobial activity towards bacteria as compared to fungii. The antibacterial activity shows following trend. 
Sal-TMP.Ar $\mathrm{ArCl}_{2} \mathrm{Te}$ Sal-TMP.ArTeCl $\mathrm{Cl}_{2} \approx$ Sal-TMP Schiff base

Sal-TMP. $\mathrm{Ar}_{2} \mathrm{TeCl}$ and Sal-TMP.ArTeCl $\mathrm{Cl}_{2}$ complexes have activity towards $S$. typhi and more effectively against $B$. cereus but Schiff base does not show activity against these bacterial strains. Schiff base and its complexes show almost similar activity against fungal strains.

Table 4. Minimum Inhibitory Concentration, MIC, $\mu \mathrm{g} / \mathrm{mL}$; (-) Resistant

\begin{tabular}{|c|c|c|c|c|c|c|c|c|c|}
\hline \multirow[b]{2}{*}{ 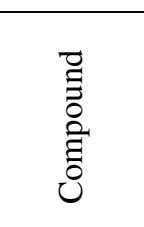 } & \multicolumn{6}{|c|}{ Bacteria strains } & \multicolumn{3}{|c|}{ Fungal strains } \\
\hline & $\begin{array}{c}S . \\
\text { aureus } \\
\text { (ATCC } \\
11632 \text { ) }\end{array}$ & $\begin{array}{l}\text { S. typhi } \\
\text { (ATCC } \\
15499 \text { ) }\end{array}$ & $\begin{array}{c}P . \\
\text { aeruginosa } \\
\text { (ATCC } \\
\text { 23564) }\end{array}$ & $\begin{array}{c}E . \\
a \quad c o l i \\
(\mathrm{ATCC} \\
35218)\end{array}$ & $\begin{array}{c}\text { B. cereus } \\
\text { (MTCC } \\
7350)\end{array}$ & $\begin{array}{c}P . \\
\text { rettgeri } \\
\text { (DRDE } \\
\text { strain) }\end{array}$ & A. niger & $\begin{array}{c}A . \\
\text { fumigates }\end{array}$ & $\begin{array}{l}\text { A. } \\
\text { flavus }\end{array}$ \\
\hline Sal-TMP & 2.5 & - & 1.25 & 5.0 & - & - & 20 & 5.0 & 1.25 \\
\hline I & 2.5 & - & 1.25 & - & 0.625 & 5.0 & - & - & - \\
\hline II & 5.0 & - & 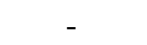 & - & 1.25 & 2.5 & 20 & 5.0 & 1.25 \\
\hline III & - & 20 & 10 & 5.0 & 1.25 & - & 5.0 & - & - \\
\hline IV & 1.25 & 2.5 & 1.25 & 5.0 & - & - & 5.0 & 10 & 5.0 \\
\hline V & - & 20 & 10 & 5.0 & 1.25 & - & 20 & - & - \\
\hline VI & 1.25 & 2.5 & 1.25 & 5.0 & - & - & - & 5.0 & - \\
\hline VII & 1.25 & - & 5.0 & 1.25 & 0.625 & 5.0 & - & 10 & - \\
\hline VIII & 2.5 & - & 1.25 & 5.0 & - & - & 20 & 5.0 & 1.25 \\
\hline
\end{tabular}

\section{Conclusion}

Aryltellurium(IV) and diaryltellurium(IV) dichlorides upon reaction with Schiff base(Sal-TMP) derived from salicylaldehyde and trimethoprim yield new complexes of tellurium(IV). The synthesized complexes were characterized by elemental analyses, conductance measurement, IR and ${ }^{1} \mathrm{H}$ NMR spectral studies. The analytical data suggest that the Sal-TMP Schiff base complexes have 1:1 stoichiometry. The Schiff bases (Sal-TMP) in these complexes functions as a uninegative tridentate ligand through azomethine nitrogen, phenolic oxygen after deprotonation and pyrimidine ring nitrogen atoms. Based on these studies, distorted octahedral geometry with two chelating rings has been assigned to these complexes. The complexes have been observed to possess substantial antimicrobial activity especially against bacteria.

\section{Acknowledgement}

The authors are grateful to M. D. University, Rohtak for providing the necessary facilities. One of the authors (Deepak) is also thankful to UGC New Delhi for providing fellowship. We also thank SAIF, Panjab University Chandigarh for providing the CHN analyses and spectral data.

\section{References}

1. Burchall J J, Corcoran J W and Hahn F E, Eds., Antibiotics, Springer, New York, NY, USA, $1975,3$.

2. Iqbal M S, Khan A H, Loothar B A and Bukhari I H, Med Chem Rev., 2009, 18, 31-42.

3. Blower P J, Аnnu Rep Prog Chem Sect A, 1999, 95, 631-655; DOI:10.1039/A808038K

4. Dhahir S A, Al-Sahib S A, Al Razaq W A and Kadhim N J, Baghdad Sci J., 2010, 7(1), 621.

5. Dr. Karl Thomae Gmb H, (Biberach an der Riss. DE), United States Patent. 4829058.

6. EL-Mossalamy E H, AL-Thabati S A, ALNomaiser F M, Commun Fac Sci Univ Ank Series B, 2005, 51(2), 21-30 
7. Sinha D, Tiwari A K, Singh S, Shukla G, Mishra P, Chandra H and Mishra A K, Eur J Med Chem., 2008, 43(1), 160-165; DOI:10.1016/j.ejmech.2007.03.022

8. Crowe A J, Smith P J and Atassi G, Chem Biol Interact., 1980, 32(1-2), 171-178; DOI:10.1016/0009-2797(80)90075-7

9. Wang M, Wang L F, Li Y Z, Li Q X, Xu Z D and Qu D M, Trans Met Chem., 2001, 26(3), 307-310; DOI:10.1023/A:1007159301849

10. Zishen Wu, Ziqi Gui and Zhenhuan Yen, Synth React Inorg Met Org Chem., 1990, 20(3), 335-344.

11. Desai S B, Desai P B and Desai K R, Hetrocycl Commun., 2001, 7(1), 83-90; DOI:10.1515/HC.2001.7.1.83

12. Dhumwad S D, Gudasi K B and Goudar T R, Indian J Chem., 1994, 33A, 320-324.

13. Przybylski P, Huczynski A, Pyta K, Brzezinski B and Bartl F, Curr Org Chem., 2009, 13(2), 124-148; DOI:10.2174/138527209787193774

14. Pandeya S N, Sriram D, Nath G and De Clercq E, Pharm Acta Hely., 1999, 74(1), 7-11.

15. Karthikeyan M S, Parsad D J, Poojary B, Bhat K S, Holla B S and Kumari N S, Bioorg. Med. Chem., 2006, 14(22), 7482-7489; DOI:10.1016/j.bmc.2006.07.015

16. Holla B S, Akberali P M and Shivananda M K, II Farmaco, 2001, 56(12), 919-927; DOI:10.1016/S0014-827X(01)01124-7

17. Jarrahpour A, Khalili D, De Clercq E, Salmi C and Brunel J M, Molecules, 2007, 12(8), 1720-1730; DOI:10.3390/12081720

18. Da Silva C M, da Silva D L, Modolo L V, Alves R B, de Resende, M A, Martins C V B and de Fatima A J, Adv Res., 2011, 2, 1-8; DOI:10.1016/j.jare.2010.05.004

19. Singh H, Yadav L D S and Mishra S B S, J Inorg Nucl Chem., 1981, 43(7), 17011704; DOI:10.1016/0022-1902(81)80367-3

20. Saravanan G, Pannerselvam P and Prakash C R, J Adv Pharm Techn Res., 2010, 1(3), 320-325; DOI:10.4103/0110-5558.72426

21. Panneerselvam P, Nair R R, Vijayalakshmi G, Subramanian E H and Sridhar S K, Eur. J. Med. Chem. 2005, 40, 225-229; DOI:10.1016/j.ejmech.2004.09.003

22. Singh N K and Singh S B, Indian J Chem., 2001, 40A, 1070-1075.

23. Walsh O M, Meegan M J, Prendergast R M, Nakib T A, Eur J Med Chem., 1996, 31, 989-1000; DOI:10.1016/S0223-5234(97)86178-8

24. Odabasoglu M, Arslan F, Ölmez H and Büyükgüngör O, Dyes and Pigments, 2007, 75(3), 507-515; DOI:10.1016/j.dyepig.2006.06.033

25. Vicini P, Geronikaki A, Incerti M, Busonera B, Poni G, Cabras C A and Colla P L, Bioorg Med Chem., 2003, 11(23), 4785-4789; DOI:10.1016/S09680896(03)00493-0

26. Pandeya S N, Sriram D, Nath G and DeClercq E, Eur J Pharm Sci., 1999, 9, 25-31.

27. Samadhiya S and Halve A, Orient J Chem., 2001, 17, 119-122.

28. Celik Ö, Ulusoy M, Tas E and Ide S, Anal Sciences, 2007, 23, x185.

29. Mahmoud M R, El-Gyar S A, Mousrafa A A and Shaker A, Polyhedron, 1987, 6(5), 1017-1020; DOI:10.1016/S0277-5387(00)80947-X

30. Qing-Yu H, Zheng-Hua M and Ya-Me Z, J Coord Chem., 1990, 21(3), 199-207; DOI:10.1080/00958979009409716

31. Cozzi P G, Chem Soc Rev., 2004, 33, 410-421; DOI:10.1039/B307853C

32. Tarafder M T H, Jin KT, Crouse K A, Ali A M. Yamin, B $M$ and Fun H K, Polyhedron, 2002, 21(25-26), 2547-2554; DOI:10.1016/S0277-5387(02)01188-9

33. Pandeya S N, Sriram D, Clercq E DE., Pannecouque C and Witvrouw M, Indian J Pharm Sci., 1998, 60(4), 207-212. 
34. Fleischman S H and Brooks C L, Proteins: Structure, Function and Genetics, 1990, 7, 52-61.

35. Suling W J, Reynolds R C, Barrow E W, Wilson L N, Piper J R and Barrow W W, J Antimicrobial Chemother., 1998, 42(6), 811-815; DOI:10.1093/jac/42.6.811

36. Gulcan M, Sonmez M and Berber I, Turkey J Chem., 2012, 36(1), 189-200.

37. Raman N, Kulandaisamy A and Jeyasubramanian K, Synth React Inorg Met Org Chem., 2001, 31(7), 1249-1270.

38. Wynne K J and Pearson P S, Inorg Chem., 1971, 10(12), 2735-2739; DOI:10.1021/ic50106a022

39. Wynne K J and Pearson P S, J Chem Soc Commun., 1970, 556-557; DOI:10.1039/C2970000556B

40. Wynne K J, Clark A J and Berg M, J Chem Soc Dalton Trans., 1972, 2370-2374; DOI:10.1039/DT9720002370

41. Clark E R, Collet A J and Naik D G, J Chem Soc Dalton Trans., 1973, 1961-1962; DOI:10.1039/DT9730001961

42. Berg M C, Diss Abstr Int., 1972, 33, 2982.

43. Srivastava T N, Singh M and Singh H B, Indian J Chem., 1982, 21A, 307-309.

44. Srivastava T N, Srivastava R C and Srivastava M, Indian J Chem., 1982, 21A, 539.

45. Srivastava T N, Srivastava R C and Srivastava V K, J Indian Chem Soc., 1983, 60, 891-892.

46. Garad M V, Polyhedron, 1985, 4(8), 1353-1355; DOI:10.1016/S0277-5387(00)86963-6

47. Verma K K and Reena, Synth React Inorg Met-Org Chem., 1999, 29(3), 499-512; DOI:10.1080/00945719909349465

48. Verma K K, Dahiya R and Soni D, Synth React Inorg Met Org Chem., 1999, 29(6), 1033-1052; DOI:10.1080/00945719909349509

49. Verma K K and Dahiya R, Synth React Inorg Met Org Chem., 1999, 29(7), 12991314; DOI:10.1080/00945719909349529

50. Verma K K and Reena, Phosphorus, Sulfur Silicon Related Elements, 1999, 148(1), 227-234; DOI:10.1080/10426509908037013

51. Verma K K and Seema, Int J Chem Sci., 2008, 6, 371-380.

52. Srivastava S, Soni D K and Gupta H S, J Indian Chem Soc., 1996, 73, 255.

53. Narwal J K, Chhabra S, Malik R K, Garg S and Verma K K, Oriental J Chem., 2013, 29, 1339-1349.

54. Chhabra S and Verma K K, J Chem Pharm Res., 2010, 2(4), 569-575.

55. Vogel A I, A Test Book of Organic Chemistry, $3^{\text {rd }}$ Edn., Longman, London, 1975.

56. Weissberger A, Ed., Technique of Organic Chemistry, Vol. 7, $2^{\text {nd }}$ Edn., Interscience Publishers, Inc. N. Y., 1967.

57. Morgan G T and Kellet R E, J Chem Soc., 1926, 1080-1088; DOI:10.1039/JR9262901080

58. Petragnani $\mathrm{N}$ and Stefani $\mathrm{H}$ A, Tellurium in Organic Chemistry, $2^{\text {nd }}$ Edn., Academic Press, London, 2007, 67, 76.

59. Bergman J, Tetrahedron, 1972, 28(12), 3323-3331; DOI:10.1016/S0040-4020(01)93674-9

60. Khandelwal B L, Kumar K and Berry F J, Inorg Chim Acta, 1981, 99(2), 135-137; DOI:10.1016/S0020-1693(00)87958-X

61. Berry F J, Kustan E H, Roshani M and Smith B C, J Organometal Chem., 1975, 99(1), 115-117; DOI:10.1016/S0022-328X(00)86367-6

62. Khandelwal B L, Kumar K and Raina K, Synth React Inorg Met Org Chem., 1981, 11, 65-78.

63. Geary W J, Coord Chem Rev., 1971, 7(1), 81-122; DOI:10.1016/S0010-8545(00)80009-0 
64. Greenwood N N, Straughan B P and Wilson A E, J Chem Soc A, 1968, 2209-2212; DOI: $10.1039 / \mathrm{J} 19680002209$

65. Srivastava K P, Singh A and Singh S K, IOSR J Appl Chem., 2014, 7(4), 16-23; DOI:10.9790/5736-07411623

66. Baker A W and Shulgin A T, J Am Chem Soc., 1959, 81(7), 1523-1529; DOI:10.1021/ja01516a001

67. Freedman H H, J Am Chem Soc., 1961, 83(13), 2900-2905; DOI:10.1021/ja01474a026

68. Flett M St C, Spectrochim Acta, 1957, 10(1), 21-37; DOI:10.1016/0371-1951(57)80160-X

69. Bellamy I J, Infrared Spectra of Complex Molecules, Chapman and Hall Ltd. London, 1975.

70. Matijević-Sosa J, Vinković M and Vikić-Topić D, Croatica Chemica Acta., 2006, 79(3), 489-495.

71. Mishra A P and Soni M, Metal Baesd Drugs, 2008, 71, 243; DOI:10.1155/2008/875410

72. Aminabhavi T M, Biradar N S and Patil C S, Inorganica Chimica Acta., 1983, 78, 107-111; DOI:10.1016/S0020-1693(00)86498-1

73. Biradar N S, Aminabhavi T M and Patil C S, Inorganica Chimica Acta., 1983, 78, 47-50; DOI:10.1016/S0020-1693(00)86487-7

74. Tumer M, Celik C, Koksal H and Serin S, Transition Metal Chem., 1999, 24(5), 525532; DOI:10.1023/A:1006982622965

75. Gwaram N S, Mohd Ali H, Khaledi H, Abdulla M A, Hamid A, Hadi A, Kawi Lin T, Ching C L and Ooi C L, Molecules, 2012, 17(5), 5952;

DOI:10.3390/molecules17055952

76. Rudzinski W E and Aminabhavi T M, Inorganica Chimica Acta., 1982, 67, 177-182; DOI:10.1016/S0020-1693(00)85061-6

77. Osowole A A, Wakil S M and Alao O K, World Appl Sci J., 2015, 33(2), 336-342; DOI:10.5829/idosi.wasj.2015.33.02.22206

78. UR-Rehman S, Faiz A and Nawaz R, J Chil Chem Soc., 2013, 58(1), 1624-1627; DOI:10.4067/S0717-97072013000100026.

79. Mohamed G G and Abd El-Wahab Z H, J Thermal Anal Calorimetry, 2003, 73(1), 347-359; DOI:10.1023/A:1025126801265

80. Verma K K, Soni D and verma, Phosphorus, Sulfur Silicon, 2000, 166(1), 231-241; DOI:10.1080/10426500008076544

81. Pant B C, McWhinnie W R and Dance N S, J Organmetal Chem., 1973, 63, 305-310; DOI:10.1016/S0022-328X(73)80043-9

82. Srivastava T N, Singh J D, Indian J. Chem., 1987, 26A, 260.

83. Chauhan S, Garg S and Verma K K, Chem Sci Trans., 2016, 5(2), 431-441; DOI:10.7598/cst2016.1193

84. Kulkarani Y D, Srivastava S, Abdi S H R and Athar M, Synth React Inorg Met Org Chem., 1985, 15(8), 1043-1059; DOI:10.1080/00945718508060634

85. Chauhan S, Garg S and Verma K K, Res J Pharm Biol Chem Sci., 2016, 7(2), 265-274.

86. Aminabhavi T M and Rudzinski W E, Inorganica Chimica Acta., 1983, 76, L131L134; DOI:10.1016/S0020-1693(00)81478-4

87. Aminabhavi T M and Rudzinski W E, Inorganica Chimica Acta., 1983, 78, 51-55; DOI:10.1016/S0020-1693(00)86488-9

88. Cappuccino J C, Sherman N, Microbiology- A Laboratory Manual, Addison Wesley, California, 1999, 263.

89. Pharmacopoeia of India, Volume 1, Controller of Publications, Ministry of Health Department, Government of India, New Delhi, 2007, 37. 